\title{
Experimental Investigation of an Aspirated Fan Stage
}

\author{
by \\ Brian Joseph Schuler
}

S.B., Massachusetts Institute of Technology (1996)

S.M., Massachusetts Institute of Technology (1998)

Submitted to the Department of Aeronautics and Astronautics

in partial fulfillment of the requirements for the degree of

Doctor of Philosophy

at the

\section{MASSACHUSETTS INSTITUTE OF TECHNOLOGY}

September 2001

(c) Massachusetts Institute of Technology 2001. All rights reserved.

Author

Department of Aeronautics and Astronautics

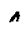

July 6,2001

Certified by ........

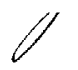

$\cap \wedge$

Certified by

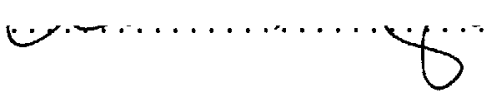

Professor of Aeronautics and Astronautics

Jack L. Kerrebrock

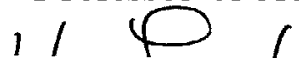

Thesis Supervisor

Alan H. Epstein

Richard Cockburn McClaurin Professor of Aeronautics and Astronautics Committee Member

Certified by .........

Mark Drela

Professor of Aeronautics and Astronautics " $\quad 1$. IOtmonittee Member

Accepted by

MASSACHUSETTS INSTITUTE OF TECHNOLOGY

AUG 132002
Wallace E. Vander Velde Chair, Committee on Graduate Students 



\title{
Experimental Investigation of an Aspirated Fan Stage
}

\author{
by \\ Brian Joseph Schuler \\ Submitted to the Department of Aeronautics and Astronautics \\ on May 18, 2001, in partial fulfillment of the \\ requirements for the degree of \\ Doctor of Philosophy
}

\begin{abstract}
This thesis focuses on the use of aspiration on compressor blade design. The pressure ratio can be significantly increased by controlling the development of the blade and endwall boundary layers. This concept is validated through an aspirated fan stage experiment performed in the MIT Blowdown Compressor Facility. The fan stage was designed to produce a pressure ratio of 1.6 at a throughflow adiabatic efficiency of $89 \%$ at a rotor tip speed of $750 \mathrm{ft} / \mathrm{s}$. Aspiration equal to $0.5 \%$ of the inlet flow was applied to the blade surface of both the rotor and stator. Aspiration was also used on the endwall boundary layers. Detailed flowfield measurements are made behind the rotor and stator, and the ensemble-averaged data is compared with a $3-\mathrm{D}$, viscous analysis tool.

The time-accurate flow measurements show a large blade to blade variation due to unsteady vortex shedding, which is not captured by conventional 3-D, viscous analysis tools. An incompressible, vortex shedding model calibrated to the experimental data shows that the vortex shedding induces radial flows that redistribute flow properties in the spanwise direction. 'Correction' of the experimental data using the model gives a better comparison with the $3-\mathrm{D}$, viscous analysis solution.

In order to understand the possible benefits of aspiration, a meanline parameter study is performed over a range of rotor inlet Mach numbers, flow coefficients, and work coefficients. Viscous and shock losses are estimated for both conventional and aspirated stages. The results suggest that aspiration can have the largest impact on compressor performance at high stage pressure ratios.
\end{abstract}

Thesis Supervisor: Jack L. Kerrebrock

Title: Professor of Aeronautics and Astronautics 


\section{Acknowledgments}

First, I would like to thank my committee members for their dedication and guidance to this work. Specifically, I would like to thank Prof. Epstein and Prof. Drela for their suggestions and critique that helped to steer this research down the path that lead to a successful doctorate. Thanks to Prof. Spearing for giving me advice and encouragement throughout the doctoral process.

Finally, thanks to my advisor, Prof. Kerrebrock. Even in my limited experience, I have to believe that it is rare to find such an intelligent and passionate person. There were countless days when he took time to come down to lab to help and, more importantly, teach me how to run a successful experiment. When the inevitable, unexplainable problems showed up, he was first to dig in to find the solution. I will be forever grateful for his kindness and dedication to my personal and professional growth.

I offer thanks to Dr. Ali Merchant and Dr. Gerry Guenette for their contributions in suffering through the reading of this thesis. Their comments and suggestions were a great help in achieving this final document.

Viktor Dubrowski deserves sincere thanks for his machining expertise that got this experiment off the ground. Jimmy Letendre provided help with the 'nuts and bolts' part of the Blowdown Compressor. Thanks to Bill Ames for his help in procurement of the necessary parts. Diana Park deserves great praise for her wonderful talent in turning my very bland graphs into interesting presentations. Not enough can be said about Lori Martinez who always had a smiling face, a word of encouragement, and, most importantly, candy. Thanks to Holly Anderson for performing the difficult task of fiscal officer.

As anyone who has done it knows, no successful trip through MIT can be completed without a set of great friends. Luis, Adam, and Anand have provided countless moments of both unfathomable depth and complete uselessness. But they always keep things interesting. Dennis, Corinne, and Malinda were the backbone of my undergraduate academic experience. Thanks for always pushing me. I'm glad too that Sarah is now here to push Dennis, he needs it. Thanks to Becky for all the lunch conversations. Hearing about your life always makes me feel better. James never let me feel that I was worse off than him. To all my former officemates and labmates, Jessica, Chris, Sumita, Chee-wei, Margarita, Sanith, thanks for all the support, encouragement, and advice, technical and personal.

My deepest and sincerest appreciation is due to my family for their unending support and encouragement, especially to my mother and father who raised me in a small Kentucky town but never allowed me to feel constrained within it. My dreams and aspirations were perpetually encouraged, and I constantly felt as if I could achieve anything. It is the teaching of this eternal optimism to which I am forever indebted.

Yet, this work could not have been completed without the unending love, patience, compassion, and perseverance of my wife, Judy. Truly, my happiest days are with you and my saddest days when we are apart. Our future and our life together is the only dream that I wish for. I know that it will be a great and epic journey. 


\section{Contents}

$\begin{array}{llr}1 & \text { Introduction and Thesis Objectives } & 17\end{array}$

1.1 Introduction . . . . . . . . . . . . . . . . . . 17

1.1.1 Previous Research . . . . . . . . . . . . . . . 18

1.1 .2 Effects of Aspiration . . . . . . . . . . . . . . . . . 19

1.2 Thesis Objectives . . . . . . . . . . . . . . . . . . . . . 22

1.3 Thesis Organization . . . . . . . . . . . . . . . . . . 23

2 Aerodynamic and Structural Design 25

2.1 Introduction . . . . . . . . . . . . . . . . . 25

2.2 Aerodynamic Design . . . . . . . . . . . . . . . . . 26

$2.2 .1 \quad$ MISES Design . . . . . . . . . . . . . . . . 26

2.2 .2 APNASA Analysis . . . . . . . . . . . . . . . 34

2.2 .3 Incidence Mismatch . . . . . . . . . . . . . . . . . 36

2.3 Mechanical Design . . . . . . . . . . . . . . . . . 38

2.3.1 Stage Construction . . . . . . . . . . . . . . . 38

2.3.2 Suction Removal System . . . . . . . . . . . . . . . . . . . . 39

2.3.3 Total Suction Requirement . . . . . . . . . . . . . . . . . 42

2.4 Summary . . . . . . . . . . . . . . . . . . . . 43

3 Experimental Setup and Data Acquisition $\quad 45$

3.1 Introduction . . . . . . . . . . . . . . . . . 45

3.2 Testing Facility $\ldots \ldots \ldots \ldots \ldots \ldots \ldots \ldots \ldots$

3.2 .1 Blowdown Compressor Operation . . . . . . . . . . . . . 46 
3.2.2 Data Collection System . . . . . . . . . . . . 47

3.3 Data Acquisition . . . . . . . . . . . . . . . . . 48

3.3.1 Wall Static Transducers . . . . . . . . . . . . . . 48

3.3 .2 Optical Encoder . . . . . . . . . . . . . . 49

3.3.3 Total and Static Probes . . . . . . . . . . . . . . 49

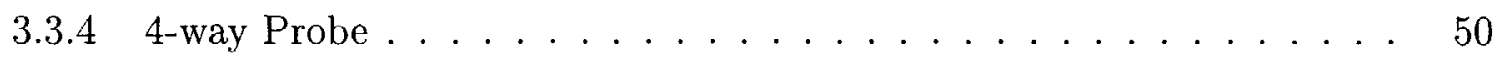

3.4 Data Acquisition System . . . . . . . . . . . . . . . . . 52

3.5 Error Analysis . . . . . . . . . . . . . . . . 52

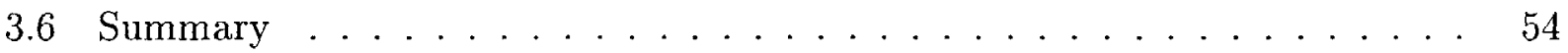

4 Aspirated Fan Stage Experiment $\quad 55$

4.1 Introduction . . . . . . . . . . . . . . . . 55

4.2 Rotor Design Point Performance . . . . . . . . . . . . . . . 56

4.3 Design Point Total Pressure and Total Temperature Spanwise Profiles . . . . 57

$4.3 .128 \% \operatorname{Span} \ldots \ldots \ldots \ldots \ldots$

$4.3 .247 \% \operatorname{Span} \ldots \ldots \ldots \ldots 59$

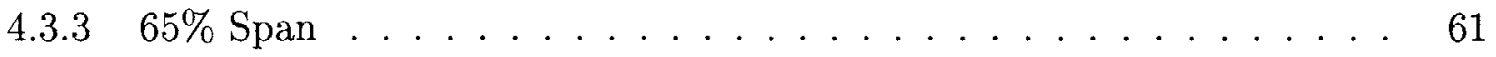

$4.3 .484 \% \operatorname{Span} \ldots \ldots \ldots \ldots \ldots$

$4.3 .592 \% \operatorname{Span} \ldots \ldots \ldots 6 . \ldots \ldots \ldots$

4.3 .6 Blade to Blade Variation . . . . . . . . . . . . . . 69

4.4 Stator Performance . . . . . . . . . . . . . . . . 72

4.5 Measured Stage Suction . . . . . . . . . . . . . . 74

4.6 Off-design Performance . . . . . . . . . . . . . . . . . . 77

4.7 Parameter View of Off-design Performance . . . . . . . . . . . 79

4.8 Summary of Experimental Results . . . . . . . . . . . . . . . . 83

5 Unsteady Radial Transport $\quad 85$

5.1 Introduction . . . . . . . . . . . . . . . . 85

5.2 Vortex Shedding and 2-D Modelling of the Rotor Wakes . . . . . . . . 86

5.3 Fitting the Vortex Parameters to Experimental Data . . . . . . . . . . 91 
5.4 Extension to Spanwise Vortex Shedding . . . . . . . . . . . . . 92

5.5 Spanwise Redistribution of Total Pressure and Total Temperature . . . . . . 99

5.5 .1 No Spanwise Mixing . . . . . . . . . . . . . . . . . 99

5.5.2 With Spanwise Mixing . . . . . . . . . . . . . . . . 101

5.6 Results of Spanwise Redistributions with and without Mixing . . . . . . 103

6 Parametric Study $\quad 105$

6.1 Introduction . . . . . . . . . . . . . . . 105

6.2 Model Development . . . . . . . . . . . . . . . 105

6.3 Compressibility and Losses . . . . . . . . . . . . . . . . . . . 106

6.3 .1 Upstream of the Rotor . . . . . . . . . . . . . . 107

6.3.2 Viscous Loss vs. Diffusion Factor Correlations . . . . . . . . . . . . . 107

6.3.3 Shock Losses . . . . . . . . . . . . . . . . . . . . 109

6.3 .4 Rotor-stator gap conditions . . . . . . . . . . . 110

6.4 Results of the Parameter Study . . . . . . . . . . . . . . . . . . 112

6.4.1 Parametric Comparison of Conventional and Aspirated Designs . . . 112

6.4 .2 Constant Mass Flow . . . . . . . . . . . . . . . . . . 115

6.4 .3 Maximum Efficiency Designs . . . . . . . . . . . 116

6.5 Meanline Parameter Study Limitations . . . . . . . . . . . . . . . . . 120

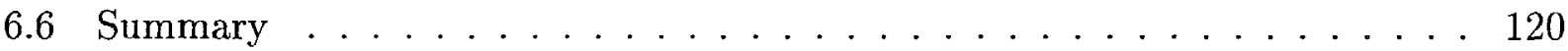

7 Stator Redesign $\quad 123$

7.1 Introduction . . . . . . . . . . . . . . . . . . . 123

7.2 Redesigned Stator without Swirl . . . . . . . . . . . . . 123

7.3 Redesigned Stator with swirl . . . . . . . . . . . . . . . . 127

7.4 Results . . . . . . . . . . . . . . . . . . 130

8 Conclusions, Contributions, and Future Work $\quad 131$

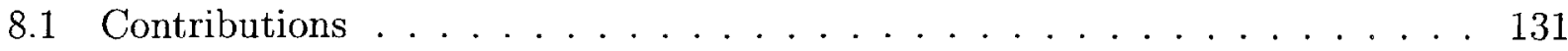

8.1.1 Design Point Experimental Results ............... 132

8.1 .2 Off-design Results . . . . . . . . . . . . . . 133 
8.1 .3 Unsteady Radial Transport . . . . . . . . . . . . . . . . 134

8.1 .4 Parameter Study . . . . . . . . . . . . . . . . . . . . . 134

8.1 .5 Stator Redesign . . . . . . . . . . . . . . . . . . 135

8.2 Future Work . . . . . . . . . . . . . . . . . 136

$\begin{array}{ll}\text { A Total and Static Pressure Probe } & 137\end{array}$

$\begin{array}{ll}\text { B New Blowdown Compressor Window Design } & 139\end{array}$

C 4-way Probe Calibration Technique and Data 143

$\begin{array}{ll}\text { D Parametric Study with Radial Variation of Streamlines } & 161\end{array}$

D.1 Introduction . . . . . . . . . . . . . . . . . . . . 161

D.1.1 Determining the Radial Variation through the Stage . . . . . . . . 161

D.1.2 Parameter Study Equation Changes . . . . . . . . . . . . . . 163

D.1.3 Comparison Plots of Parameter Study with Compressor Data . . . 165

D.1.4 Results with Radial Variations and Axial Velocity Ratio . . . . . 167

D.2 Results . . . . . . . . . . . . . . . . . . . . . 176 


\section{List of Figures}

1-1 Effect of aspiration on downstream boundary layer growth $\ldots \ldots 20$

1-2 Highly loaded transonic rotor blade row without suction $\ldots \ldots . \ldots 21$

1-3 Highly loaded transonic rotor blade row with suction $\ldots \ldots \ldots 22$

2-1 Blade Shape and Mach number distribution for the rotor tip section . . . . 27

2-2 Blade shape and Mach number distribution for the stator hub section . . . 28

$2-3$ Rotor inlet relative mach number from APNASA . . . . . . . . . . 29

$2-4$ Rotor inlet relative flow angle from APNASA $\ldots \ldots \ldots$

$2-5$ Rotor exit relative mach number from APNASA . . . . . . . . . . . . 30

2-6 Rotor exit relative flow angle from APNASA . . . . . . . . . . . . . 31

2-7 Stator inlet absolute mach number from APNASA . . . . . . . . . . . 31

2-8 Stator inlet absolute flow angle from APNASA . . . . . . . . . . . . 32

2-9 Stator exit absolute mach number from APNASA . . . . . . . . . . . 32

$2-10$ Stator exit absolute flow angle from APNASA $\ldots \ldots \ldots 33$

2-11 Total pressure at rotor exit from APNASA analysis $\ldots \ldots \ldots 33$

2-12 Total temperature at rotor exit from APNASA analysis . . . . . . . . . 34

2-13 Total pressure at stator exit from APNASA analysis . . . . . . . . . . 35

2-14 Total temperature at stator exit from APNASA analysis . . . . . . . . 35

2-15 Total Pressure Contours from APNASA analysis . . . . . . . . . . . 36

2-16 Assembly cross-section and suction scheme . . . . . . . . . . . . . 38

2-17 Rotor blade with and without cover plate . . . . . . . . . . . 40

2-18 Rotor Shroud with Blade Suction Choke Holes . . . . . . . . . . . . . 41 
3-1 Blowdown Compressor Schematic . . . . . . . . . . . . . . 46

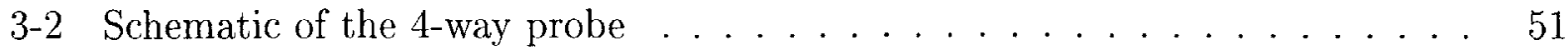

4-1 Comparison of experimental spanwise total pressure distribution with AP-

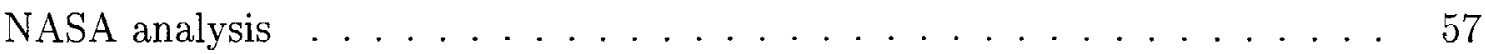

4-2 Comparison of experimental spanwise total temperature distribution with AP-

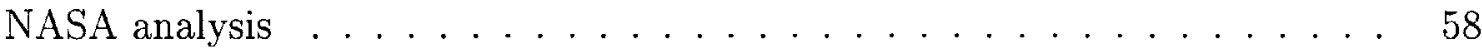

4-3 Pitchwise Total Pressure Profile for $28 \%$ Rotor Span . . . . . . . . . . . 59

4-4 Pitchwise Tangential Angle Profile for $28 \%$ Rotor Span . . . . . . . . . . . 60

4-5 Time-Accurate Total Pressure and Tangential Angle for 28\% Rotor Span . . 60

4-6 Pitchwise Total Pressure Profile for $47 \%$ Rotor Span . . . . . . . . . . . 61

4-7 Pitchwise Tangential Angle Profile for 47\% Rotor Span . . . . . . . . . . 62

4-8 Time-Accurate Total Pressure and Tangential Angle for 47\% Rotor Span . . 62

4-9 Pitchwise Total Pressure Profile for 65\% Rotor Span . . . . . . . . . . 63

4-10 Pitchwise Tangential Angle Profile for 65\% Rotor Span . . . . . . . . . . . . 64

4-11 Time-Accurate Total Pressure and Tangential Angle for 65\% Rotor Span . . 64

4-12 Pitchwise Total Pressure Profile for $84 \%$ Rotor Span . . . . . . . . . . 65

4-13 Pitchwise Tangential Angle Profile for 84\% Rotor Span . . . . . . . . . . . . 66

4-14 Time-Accurate Total Pressure and Tangential Angle for 84\% Rotor Span . . 67

4-15 Pitchwise Total Pressure Profile for $92 \%$ Rotor Span . . . . . . . . . . 68

4-16 Pitchwise Tangential Angle Profile for 92\% Rotor Span . . . . . . . . . 68

4-17 Time-Accurate Total Pressure and Tangential Angle for 92\% Rotor Span . . 69

4-18 Blade to Blade Variation in the Total Pressure data for $28 \%$ Rotor Span . . 70

4-19 Blade to Blade Variation in the Total Pressure data for $60 \%$ Rotor Span . . 71

4-20 Blade to Blade Variation in the Total Pressure data for $92 \%$ Rotor Span . . 71

4-21 Stator exit total pressure contour predicted by APNASA . . . . . . . . 73

4-22 Time-averaged total pressure data from stator exit . . . . . . . . . . . . 73

4-23 Time accurate measurement of rotor casing suction . . . . . . . . . . . 75

4-24 Time accurate measurement of rotor blade suction . . . . . . . . . . . 75

$4-25$ Time accurate measurement of stator suction . . . . . . . . . . . 76 
4-26 Off-design performance of Aspirated Fan Stage . . . . . . . . . . . . 77

4-27 Pitchwise Total Pressure at Rotor Exit for 65\% Span at different Mass Flows 78

4-28 Un-averaged total pressure data at $100 \%$ and $79 \%$ Mass Flow . . . . . . 78

4-29 Stator hub section diffusion factor for $79 \%$ and $100 \%$ design mass flow . . . 79

4-30 Stator tip section diffusion factor for $79 \%$ and $100 \%$ design mass flow . . . 80

4-31 Stator hub section inlet Mach number for $79 \%$ and $100 \%$ design mass flow . 81

4-32 Stator tip section inlet Mach number for $79 \%$ and $100 \%$ design mass flow . . 81

4-33 Stator hub inlet tangential flow angle for $79 \%$ and $100 \%$ design mass flow . . 82

4-34 Stator hub inlet tangential flow angle for $79 \%$ and 100\% design mass flow . . 82

5-1 Ensemble-averaged wake profile from the LA measurements from [4] . . . 88

5-2 Velocity probability density for different points within the wake from [4] . . 89

5-3 Schematic of the von Karmen vortex street model used by Gertz [4] . . . . 90

5-4 Schematic of the flow regions used in the calculation of spanwise redistribution of flow properties . . . . . . . . . . . . . . . . . . . . . . . 94

5-5 Time-accurate radial Mach number distribution at $92 \%$ span $\ldots \ldots . \ldots 96$

$5-6$ Radial Mach number distribution from APNASA at $92 \%$ span . . . . . . 96

5-7 Time-accurate radial Mach number distribution at $65 \%$ span $\ldots \ldots$. . . . 97

$5-8$ Radial Mach number distribution from APNASA at $65 \%$ span . . . . . . 97

5-9 Time-accurate radial Mach number distribution at $28 \%$ span . . . . . . . . 98

5-10 Radial Mach number distribution from APNASA at $28 \%$ span . . . . . . 98

5-11 Idealized view of the pitchwise flow properties at the rotor exit . . . . . . 100

$5-12$ Effect of spanwise transport on adiabatic efficiency distribution . . . . . . . 103

6-1 Loss correlation used for standard and aspirated stage design . . . . . . . . 108

6-2 Comparison of Meanline Parameter Study to Compressor Data - Stage Efficiency 113

6-3 Comparison of Meanline Parameter Study to Compressor Data - Stator Inlet Mach Number . . . . . . . . . . . . . . . . . . . . . . . . . 114

6-4 Stage performance over the given parameter range for $M_{x}=0.65 \ldots \ldots 115$

6-5 Maximum efficiency points for a range of inlet axial Mach numbers . . . . 117

6-6 Rotor diffusion factor for the maximum efficiency points . . . . . . . . 117 
6-7 Stator diffusion factor for the maximum efficiency points . . . . . . . . 118

6-8 Stator inlet Mach number for the maximum efficiency points . . . . . . . . 119

6-9 Blade loading $(\psi)$ for the maximum efficiency points $\ldots \ldots \ldots$. . . . . 119

7-1 Blade surface Mach number distribution for the hub section without exit swirl 124

7-2 Mach number contour for the hub section without exit swirl . . . . . . 125

7-3 Suction surface boundary layer growth for the hub section without exit swirl 126

7-4 Boundary layer shape factor for the hub section without exit swirl . . . . . 126

7-5 Blade surface Mach number distribution for the hub section with exit swirl . 127

7-6 Mach number contour for the hub section with exit swirl . . . . . . 128

7-7 Suction surface boundary layer growth for the hub section with exit swirl . . 129

7-8 Boundary layer shape factor distribution for the hub section with exit swirl . 129

A-1 Mechanical drawing of total and static pressure probe . . . . . . . . 138

B-1 New Window for the Blowdown Compressor . . . . . . . . . . . . . 140

B-2 Slide Plate for BDC Window . . . . . . . . . . . . . . . . . . 141

C-1 $F_{23}, C_{p 4}, C_{p 1}, K_{p 2}, K_{p 3}$, and $H_{23}$ as function of $\theta$ and $\phi$ for $\mathrm{M}=0.10 \ldots 147$

C-2 $F_{23}, C_{p 4}, C_{p 1}, K_{p 2}, K_{p 3}$, and $H_{23}$ as function of $\theta$ and $\phi$ for $\mathrm{M}=0.32 \ldots 148$

C-3 $F_{23}, C_{p 4}, C_{p 1}, K_{p 2}, K_{p 3}$, and $H_{23}$ as function of $\theta$ and $\phi$ for $\mathrm{M}=0.41 \ldots 149$

C-4 $F_{23}, C_{p 4}, C_{p 1}, K_{p 2}, K_{p 3}$, and $H_{23}$ as function of $\theta$ and $\phi$ for $\mathrm{M}=0.51 \ldots 150$

C-5 $F_{23}, C_{p 4}, C_{p 1}, K_{p 2}, K_{p 3}$, and $H_{23}$ as function of $\theta$ and $\phi$ for $\mathrm{M}=0.60 \ldots 151$

C-6 $F_{23}, C_{p 4}, C_{p 1}, K_{p 2}, K_{p 3}$, and $H_{23}$ as function of $\theta$ and $\phi$ for $\mathrm{M}=0.70 \ldots 152$

C-7 $F_{23}, C_{p 4}, C_{p 1}, K_{p 2}, K_{p 3}$, and $H_{23}$ as function of $\theta$ and $\phi$ for $\mathrm{M}=0.92 \ldots 153$

C-8 $F_{23}, C_{p 4}, C_{p 1}, K_{p 2}, K_{p 3}$, and $H_{23}$ as function of $\theta$ and $\phi$ for $\mathrm{M}=0.10 \ldots 154$

C-9 $F_{23}, C_{p 4}, C_{p 1}, K_{p 2}, K_{p 3}$, and $H_{23}$ as function of $\theta$ and $\phi$ for $\mathrm{M}=0.32 \ldots 155$

C-10 $F_{23}, C_{p 4}, C_{p 1}, K_{p 2}, K_{p 3}$, and $H_{23}$ as function of $\theta$ and $\phi$ for $\mathrm{M}=0.41 \ldots 156$

C-11 $F_{23}, C_{p 4}, C_{p 1}, K_{p 2}, K_{p 3}$, and $H_{23}$ as function of $\theta$ and $\phi$ for $\mathrm{M}=0.51 \ldots 157$

C-12 $F_{23}, C_{p 4}, C_{p 1}, K_{p 2}, K_{p 3}$, and $H_{23}$ as function of $\theta$ and $\phi$ for $\mathrm{M}=0.58 \ldots 158$

C-13 $F_{23}, C_{p 4}, C_{p 1}, K_{p 2}, K_{p 3}$, and $H_{23}$ as function of $\theta$ and $\phi$ for $\mathrm{M}=0.70 \ldots 159$

C-14 $F_{23}, C_{p 4}, C_{p 1}, K_{p 2}, K_{p 3}$, and $H_{23}$ as function of $\theta$ and $\phi$ for $\mathrm{M}=0.92 \ldots 160$ 
D-1 Diagram of an uncambered, 2-D airfoil with passage shock . . . . . . 164

D-2 Stator Inlet Mach Number Comparison of Parameter Study with Compressor

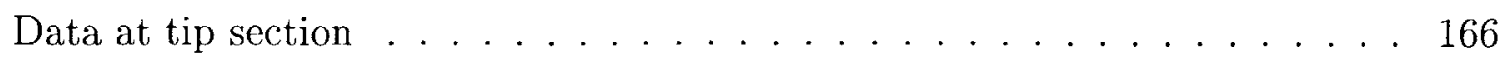

D-3 Stator Inlet Mach Number Comparison of Parameter Study with Compressor Data at hub section . . . . . . . . . . . . . . 166

D-4 Rotor Diffusion Factor Comparison of Parameter Study with Compressor Data

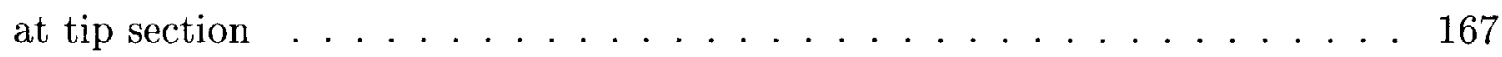

D-5 Hub Streamline for conventional and aspirated fan . . . . . . . . . . 168

D-6 Midspan Streamline for conventional and aspirated fan . . . . . . . . . 169

D-7 Tip Streamline for conventional and aspirated fan . . . . . . . . . 170

D-8 Rotor Diffusion Factor for the Tip Section . . . . . . . . . . . . 171

D-9 Rotor Diffusion Factor for the Hub Section . . . . . . . . . . . . . 172

D-10 Stator Diffusion Factor for the Tip Section . . . . . . . . . . 172

D-11 Stator Diffusion Factor for the Hub Section . . . . . . . . . . . . . 173

D-12 Stator Inlet Mach number for the Tip Section . . . . . . . . . . . 173

D-13 Stator Inlet Mach number for the Hub Section . . . . . . . . . . . . . . . . 174

D-14 Rotor Reaction for the Tip Section . . . . . . . . . . . . . . . . 174

D-15 Rotor Reaction for the Hub Section . . . . . . . . . . . . . . . 175 


\section{List of Tables}

2.1 Low speed stage design characteristics predicted by MISES . . . . . . . . 27

2.2 Low speed stage design characteristics predicted by APNASA . . . . . . 28

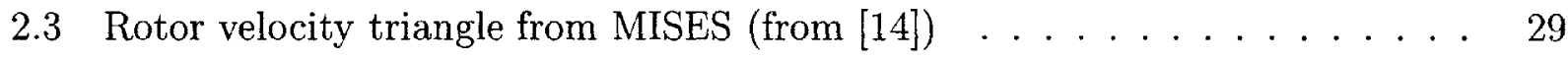

2.4 Rotor Suction Requirements . . . . . . . . . . . . . . . . . 42

2.5 Stator Suction Requirements . . . . . . . . . . . . . . . . 42

3.1 Results of the error analysis . . . . . . . . . . . . . . 54

4.1 Rotor Suction Requirements . . . . . . . . . . . . . . . . . 74

5.1 Vortex Street Parameters for the NASA LeRC Stage 67 . . . . . . . . . 87

5.2 Vortex Street Parameters for the Aspirated Fan Stage . . . . . . . . . . . . . 91

5.3 Spanwise flow values . . . . . . . . . . . . . . . . 94

D.1 Parameter study values used to create comparison plots. . . . . . . . . 165 


\section{Chapter 1}

\section{Introduction and Thesis Objectives}

\section{$1.1 \quad$ Introduction}

The figures of merit for performance of a compressor stage are pressure ratio and efficiency at the same blade speed. An increase in either quantity is considered a benefit to the compression system and overall engine performance. An increase in the pressure ratio of a given stage benefits the overall weight of the engine, since fewer stages are needed to achieve the design overall compressor pressure ratio. In current gas turbine engines, the axial compressor accounts for a large percentage of the overall length of the engine, and therefore an important fraction of the weight of the engine. Fewer stages allow a shorter compressor section with fewer compressor stages and fewer peripherals associated with the compressor. Improved compressor efficiency translates directly to an increase in the overall efficiency of the engine that, in turn, leads to lower specific fuel consumption.

Compressor performance is limited by the boundary layer performance within the compressor stage. Blade and endwall boundary layer separation limits the stage pressure ratio, and the size of the trailing edge boundary layer contributes significantly to the stage losses and mass flow. Therefore, careful removal of the boundary layer fluid within the compressor stage can produce stages with both higher pressure ratio and higher efficiencies compared with conventional stages. This thesis presents the first experimental results of a fully aspirated fan stage, how aspiration can be used to design high work compressor stages, and the 
design of a high efficiency fan stator.

\subsubsection{Previous Research}

The majority of the research aimed at increasing the pressure ratio of a compressor stage has focused on increasing the blade speed. For kinematically similar flows, the temperature rise increases as the square of the blade speed. One of the most notable examples is by Wennerstrom [26] in 1971. This stage produced a pressure ratio of 1.95 with a peak efficiency of $90 \%$ at a tip speed of $1500 \mathrm{ft} / \mathrm{s}$. Other attempts have been made to increase the pressure ratio including splittered vanes, again by Wennerstrom [27], and later improved upon by Sehra [24].

Boundary layer control has also been investigated by Loughery et al [11]. Suction and blowing was applied to high lift stator blades in a variety of configurations including single, double, and triple suction slots and single and double blowing slots. The experiments indicated that suction was more effective at controlling the boundary layer than blowing. The aspiration produced improvements in both the exit turning angle and the efficiency of the stator blades.

More recent research focused on aspiration for boundary layer control has been conducted at MIT. Several numerical designs for an aspirating compressor have been presented in recent

years and one experimental investigation has been performed. Smilg [22] used the quasi-3D, design tool MISES [1], to provide a preliminary design of an aspirated compressor stage that produced a pressure ratio of 2.0 at a tip speed of $1000 \mathrm{ft} / \mathrm{s}$ and predicted a suction flow requirement as high as $8 \%$ of the inlet flow for a single blade row, although some of the blade sections did not produce converged solutions. Similarly, Ziminsky [29] used MISES to provide a preliminary design of an aspirated compressor stage that produced a pressure ratio of 3.0 at a tip speed of $1500 \mathrm{ft} / \mathrm{s}$. This design predicted a $2.5 \%$ suction requirement for the rotor and $3.5 \%$ suction for the stator but also contained some unconverged blade sections. An experimental investigation into the effects of suction on an existing rotor was performed by Reijnen [17]. In this experiment, suction was applied to 5 blades on an existing 23-bladed rotor. The experiment showed that suction has a more significant effect on the 
flow when the blades are highly loaded. The investigation also concluded that suction would yield significant benefits only as an integral part of blade designs.

The most recent investigation into aspirated compressors was performed by Merchant [14]. Merchant provides a detailed design and analysis of two fully aspirated compressors stages. One stage produces a pressure ratio of 1.6 at a tip speed of $750 \mathrm{ft} / \mathrm{s}$, and a second stage produces a pressure ratio of 3.5 at a tip speed of $1500 \mathrm{ft} / \mathrm{s}$. The low speed stage requires removal of $1.0 \%$ of the inlet flow, and the high speed stage has a suction flow requirement

of $1.5 \%$ for the rotor and $2.0 \%$ for the stator. The low speed stage is the subject of the experimental portion of this thesis.

Kerrebrock et al. [8] have shown that aspiration can provide a thermodynamic benefit for the entire compression system. The boundary layer suction removes some of the high entropy fluid from the main flow of the compressor and lowers the entropy of the flow entering the next compression system. For a series of aspirated stages working as a compression system, removal of the high entropy boundary layer in the first stages allows the latter stages to do less work to achieve the desired total pressure rise. An adiabatic efficiency increase of four tenths of one percent is predicted for every percent of mass flow removed for a blade relative Mach number of 1.5.

\subsubsection{Effects of Aspiration}

Kerrebrock et al. [8] present a boundary layer argument that shows aspiration can have a beneficial effect on the downstream boundary layer behavior and gives qualitative insight into where aspiration would have the most benefit. The effects on a compressor stage flowpath can be shown by application of the von Karman integral momentum equation (1.1), where $\theta$ is the momentum thickness, $C_{f}$ is the friction coefficient, $H$ is the shape parameter, $M_{e}$ is the edge Mach number, $u_{e}$ is the edge velocity, $\rho_{e} u_{e}$ is the edge mass flux, and $\rho_{w} u_{w}$ is the mass flux through the wall.

$$
\frac{d \theta}{d s}=\frac{C_{f}}{2}-\left(2+H-M_{e}^{2}\right) \frac{\theta}{u_{e}} \frac{d u_{e}}{d s}+\frac{\rho_{w} v_{w}}{\rho_{e} u_{e}}
$$


In figure 1-1, suction $\left(-\rho_{w} v_{w}\right)$ is applied between $s_{1}$ and $s_{2}$ and then the boundary layer develops downstream as normal. $\Delta \theta_{2}$ is the momentum thickness decrease immediately downstream of the suction region due to the applied suction while $\Delta \theta(s)$ is the difference between the momentum thickness that develops with and without suction.

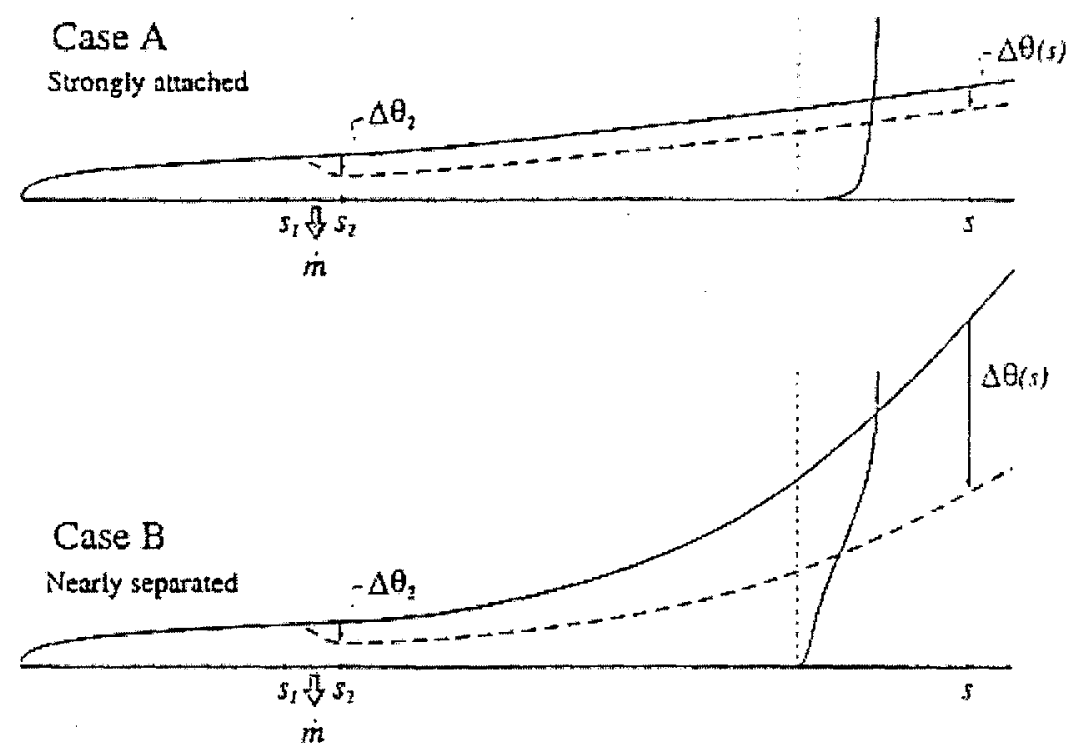

Figure 1-1: Effect of aspiration on downstream boundary layer growth

Equation (1.1) says that suction can affect the downstream boundary layer to varying degrees depending on the main flow conditions. Figure 1-1 illustrates two limiting examples:

A) Strongly-attached flow

$$
\begin{array}{r}
\frac{d \theta}{d s} \cong \frac{C_{f}}{2} \\
\theta(s) \cong \theta_{2}+\int_{s_{2}}^{s} \frac{C_{f}}{2} d s \\
\Delta \theta(s) \cong \Delta \theta_{2}
\end{array}
$$

\section{B) Nearly-separated flow}

$$
\begin{array}{r}
\frac{d \theta}{d s} \cong-\left(2+H-M_{e}^{2}\right) \frac{\theta}{u_{e}} \frac{d u_{e}}{d s} \\
\theta(s) \cong \theta_{2} \exp \left[\int_{s_{2}}^{s}-\left(2+H-M_{e}^{2}\right) \frac{1}{u_{e}} \frac{d u_{e}}{d s} d s\right]
\end{array}
$$




$$
\Delta \theta(s) \cong \Delta \theta_{2} \exp \left[\int_{s_{2}}^{s}-\left(2+H-M_{e}^{2}\right) \frac{1}{u_{e}} \frac{d u_{e}}{d s} d s\right]
$$

The first important thing to notice from these equations is that, in the strongly-attached case, the reduction of the momentum thickness is nearly constant downstream of the suction point. But in the nearly-separated flow, the suction has an exponential effect on the downstream momentum thickness. This leads to the conclusion that suction will have its greatest impact on the flow if applied just upstream of regions of high adverse pressure gradients, commonly found at shock boundaries and on the suction surface of compressor blades.

Figures 1-2 and 1-3 shows the effect of suction on a highly loaded transonic rotor. The flowfield for both airfoils was calculated using MISES [1]. The inlet relative Mach number is 1.2. The rotor on the left shows the airfoil with $1 \%$ suction applied just downstream of the shock impingement location on the suction side of the airfoil. The airfoil in figure 1-2 has no suction. Consequently, it undergoes a massive separation downstream of the shock impingement point. The airfoil in figure 1-3 has suction and shows no separated region. The overall losses of the unaspirated airfoil increase by a factor of three compared to the losses associated with the airfoil with suction. Also, the viscous losses of the airfoil without suction increase by a factor of ten.

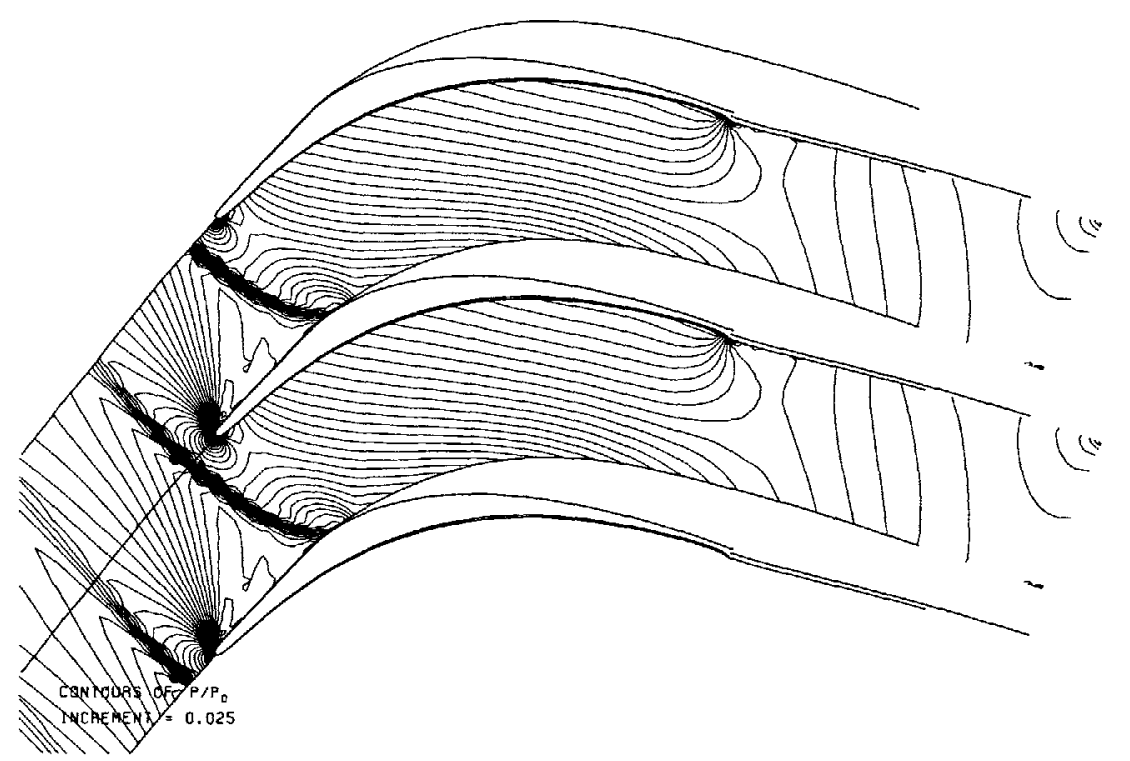

Figure 1-2: Highly loaded transonic rotor blade row without suction 


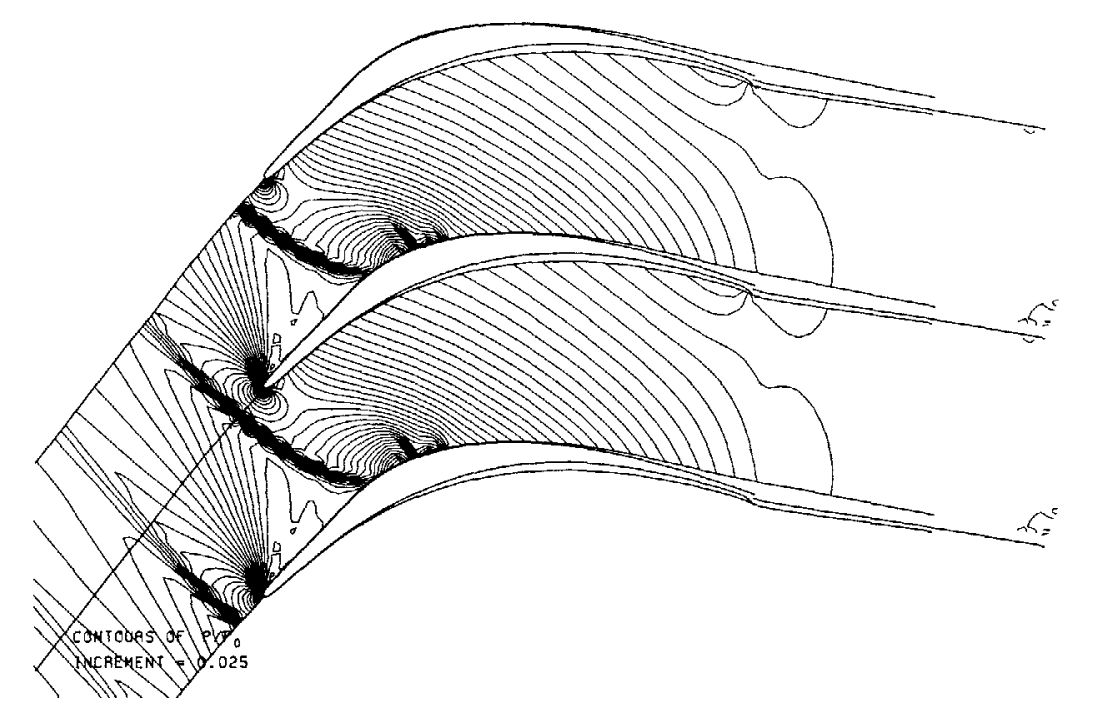

Figure 1-3: Highly loaded transonic rotor blade row with suction

\subsection{Thesis Objectives}

Aspirated compressor designs have indicated that high work, high efficiency stages can be realized with aspiration, justifying the need to experimentally verify their performance. The primary objective of this research is to experimentally examine a fully aspirated compressor, by construction and testing of the low speed design offered by Merchant[14]. The experiment focuses on the effect of suction on the main flow through the compressor, therefore less emphasis will be placed on the means for handling the aspirated flow to optimize overall system performance. The fan stage testing is performed in the Blowdown Compressor facility with emphasis on the design point operation. Off-design performance is also investigated. Results obtained from these experiments can be used to shed light on application of this technology and for comparison with $3 \mathrm{D}$, viscous CFD analyses. These comparisons provide validation of the suction schemes used in these codes.

The aspirated compressor design is more complicated than conventional compressor design because the blade suction is another dimension in the design space. It is important to compare the aspirated compressor design space to conventional compressor design space. Therefore, a secondary objective of this thesis is to examine the design conditions in which aspirated compressors could have the most benefit. A hub, tip, and meanline parameter study is performed to map aspirated compressor performance over a range of work factors 
and flow coefficients, and aspirated compressor performance is compared to conventional compressor performance for a variety of parameters including throughflow efficiency, pressure ratio, diffusion factor, flowfield Mach numbers, and rotor reaction. The viscous and shock losses are estimated, and a throughflow efficiency for the stage is determined.

The final objective of this research is to produce a design that is attractive as a fan stage for a civil turbofan engine. The design requirements for such a fan stage would be a pressure ratio of 1.6 with an adiabatic efficiency of $92 \%$. If this throughflow stage efficiency can be achieved, the lower blade speeds will offer the additional benefits of lower fan weight and noise.

\subsection{Thesis Organization}

Chapter 2 shows the detailed aerodynamic and structural designs of the aspirated fan stage that has been the subject of the experimental testing. Chapter 3 introduces the experimental setup which includes the various types of probes and pressure transducers used to gather the flowfield data. This chapter also presents an error analysis of the measurements. Chapter 4 presents the experimental data from the design point and off-design tests and compares the results with the APNASA calculation and with the parameter studies. Chapter 5 presents a vortex shedding model that is fit to the experimental data and offers an explanation for the discrepancy in spanwise variation of flow quantities between the measured data and the APNASA analysis. Chapter 6 introduces a parameter study where the simplified equations relating the parameters of compressor stage performance are modified to accommodate aspirated compressors and provide a direct comparison of aspirated designs and conventional compressor designs. Chapter 7 explores a redesign of the stator blade row with the goal of achieving a $92-94 \%$ isentropic efficiency for the stage. Then conclusions, contributions, and a discussion of future work are presented in chapter 8 . 


\section{Chapter 2}

\section{Aerodynamic and Structural Design}

\subsection{Introduction}

This chapter presents overviews of the aerodynamic and structural designs of the aspirated stage. Important aspects of the quasi-3D MISES designs and results from the 3D, viscous APNASA analysis show the basics of the aerodynamic intent. Radical blade shapes not found in conventional compressors were designed due to the high work factors involved. All of the sections below $75 \%$ span turn the flow past axial, whereas in conventional compressors, only the blade sections very near the hub turn the flow past axial. The aspirated fan stage is a new design that runs in an operating space far from that of conventional compressors.

The mechanical aspects of the aspirated fan stage experiment required new techniques for rotor and stator cover plate attachment, modifications to the Blowdown Compressor facility to accommodate the suction flows, and new measurement probes to measure the suction flow rates. Dealing effectively with the suction flows is challenging for an experiment that requires the suction flows to be constant over the test time. An elaborate suction channel design that runs through the casing insert along with choke holes for individual blades allows the suction to be measured easily and ensures that the suction is working during the required experimental time. 


\section{$2.2 \quad$ Aerodynamic Design}

The design procedures and analysis tools used to create the low speed aspirated compressor are presented in this section. The global design objectives for the low speed aspirated compressor design are a total pressure ratio for the stage of 1.60 with $0.5 \%$ suction per blade row. The goals are to lower the blade speed and to increase throughflow efficiency compared to conventional designs. This design pressure ratio goal is chosen because it is typical for today's high bypass ratio commercial engines.

\subsubsection{MISES Design}

The aspirated fan stage was designed using the quasi-3D MISES integral boundary layer solver coupled with an axisymmetric solver. The details of the design can be found in reference [14]. Figures 2-1 and 2-2 show the blade shape and Mach number distribution for the two most highly loaded blade sections, the rotor tip and the stator hub. Figure 2-1 shows the rotor tip section, and figure 2-2 shows the stator hub.

Tables 2.1 and 2.2 shows the important features of the low speed fan stage. The rotor is designed to produce a pressure ratio of 1.65 with $97 \%$ efficiency at a tip speed of $750 \mathrm{ft} / \mathrm{s}$ at standard day conditions $\left(P_{0}=1.013 * 10^{5} \mathrm{~Pa}\right.$ and $\left.T_{0}=300 \mathrm{~K}\right)$. The MISES stage produces a pressure ratio of 1.63 with $94 \%$ efficiency, while the APNASA anaylsis predicts a pressure ratio fo 1.595 at $89 \%$ efficiency. The rotor has a completely subsonic relative velocity profile, while the stator has a supersonic inlet flow on the lower half of the blade row. The maximum diffusion factors occur at the rotor tip with a value of 0.56 and at the stator hub with a value of 0.54 . Finally, the work coefficient predicted by MISES is 0.86 which is roughly twice the value of current conventional stage designs. The APNASA analysis predicts a value of 0.88 for the work coefficient.

The detailed velocity triangles between the MISES design and the APNASA analysis by comparing table 2.3 and figures 2-3-2-10. 


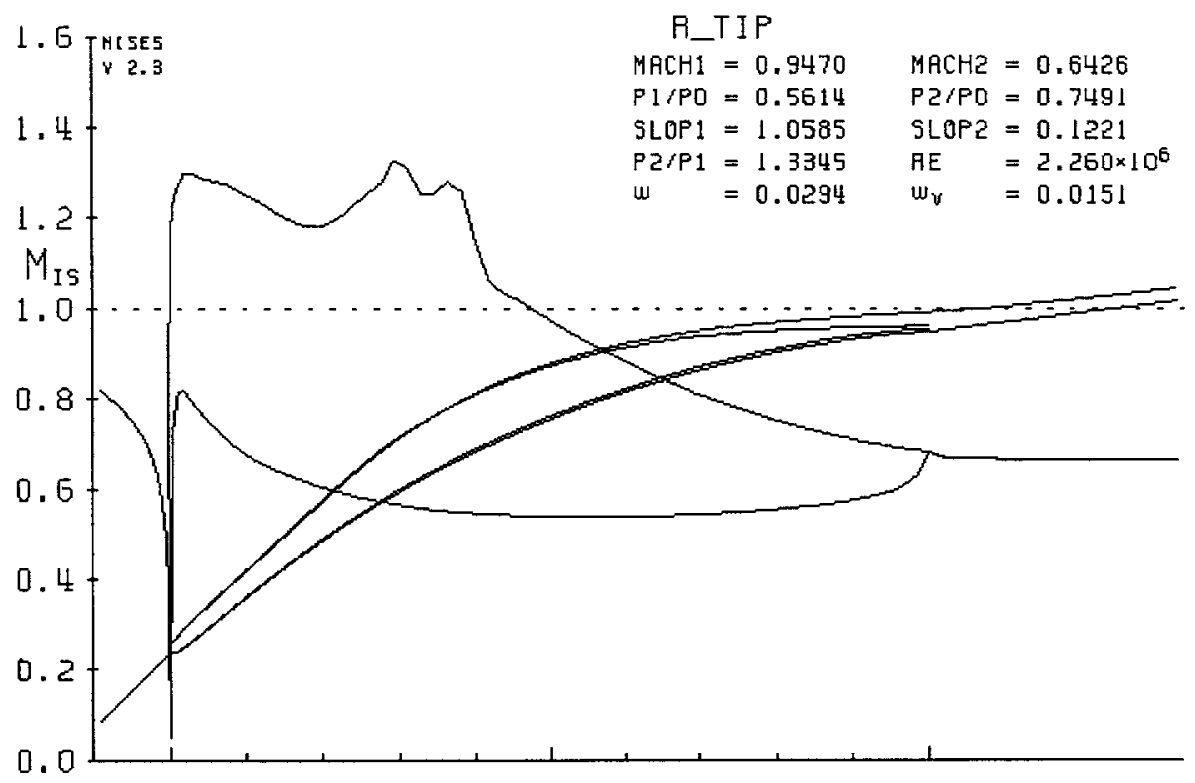

Figure 2-1: Blade Shape and Mach number distribution for the rotor tip section

Table 2.1: Low speed stage design characteristics predicted by MISES

\begin{tabular}{||l|c|}
\hline Blade Tip Speed (ft/s) & 750 \\
\hline Rotor Pressure Ratio & 1.65 \\
\hline Stage Pressure Ratio & 1.63 \\
\hline Mass Flow/Area $\left(\mathrm{lbm} / \mathrm{ft}^{2} / \mathrm{s}\right)$ & 44.8 \\
\hline Rotor face axial Mach number & 0.62 \\
\hline Stator exit Mach number & 0.70 \\
\hline Rotor tip relative Mach number & 0.95 \\
\hline Stator hub absolute Mach number & 1.15 \\
\hline Rotor Hub/Tip Ratio & 0.63 \\
\hline Suction mass (rotor/stator \%) & $0.5 / 0.5$ \\
\hline Maximum diffusion factor & $0.56 / 0.54$ \\
\hline Rotor tip solidity & 1.4 \\
\hline Stator hub solidity & 2.1 \\
\hline Rotor Isentropic Efficiency & 0.97 \\
\hline Stage Isentropic Efficiency & 0.94 \\
\hline Tip Blade Loading $\left(\Delta H / U_{\text {tip }}^{2}\right)$ & 0.86 \\
\hline
\end{tabular}




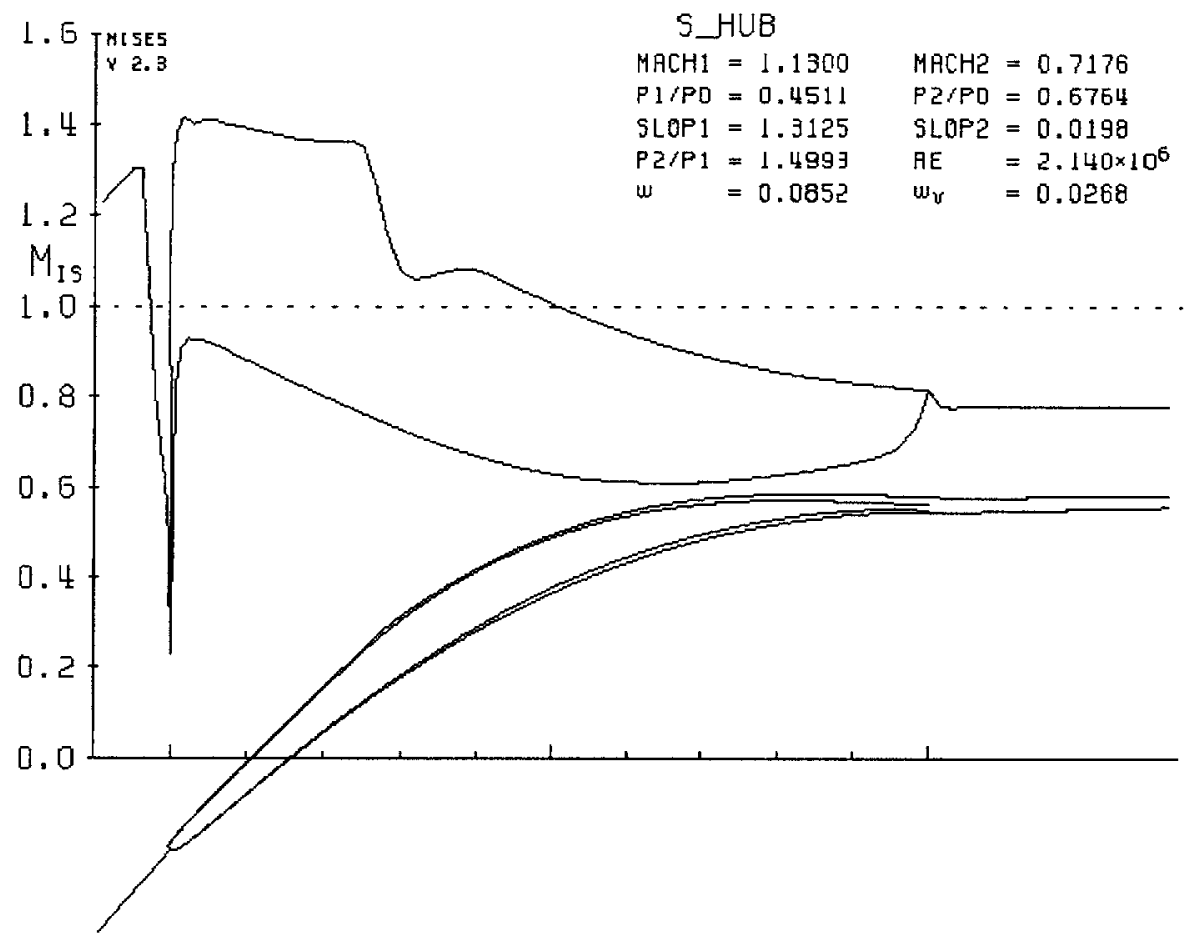

Figure 2-2: Blade shape and Mach number distribution for the stator hub section

Table 2.2: Low speed stage design characteristics predicted by APNASA

\begin{tabular}{||l|c|}
\hline Blade Tip Speed (ft/s) & 750 \\
\hline Rotor Pressure Ratio & 1.648 \\
\hline Stage Pressure Ratio & 1.595 \\
\hline Mass Flow (lb/s) & 64.0 \\
\hline Rotor Isentropic Efficiency & 0.96 \\
\hline Stage Isentropic Efficiency & 0.89 \\
\hline Tip Blade Loading $\left(\Delta H / U_{\text {tip }}^{2}\right)$ & 0.88 \\
\hline
\end{tabular}


Table 2.3: Rotor velocity triangle from MISES (from [14])

\begin{tabular}{||l|c|c|}
\hline Blade Blade Section & MISES Rel Mach & MISES angle (deg) \\
\hline Rotor hub inlet & .702 & 33.6 \\
Rotor hub exit & .835 & -33.3 \\
Rotor mid inlet & .780 & 41.8 \\
Rotor mid exit & .640 & -16.9 \\
Rotor tip inlet & .947 & 46.1 \\
Rotor tip exit & .642 & 1.8 \\
\hline Stator hub inlet & 1.13 & -52.4 \\
Stator hub exit & .72 & -1.0 \\
Stator mid inlet & 1.05 & -48.6 \\
Stator mid exit & .63 & -1.0 \\
Stator tip inlet & .89 & -44.5 \\
Stator tip exit & .58 & -0.8 \\
\hline
\end{tabular}

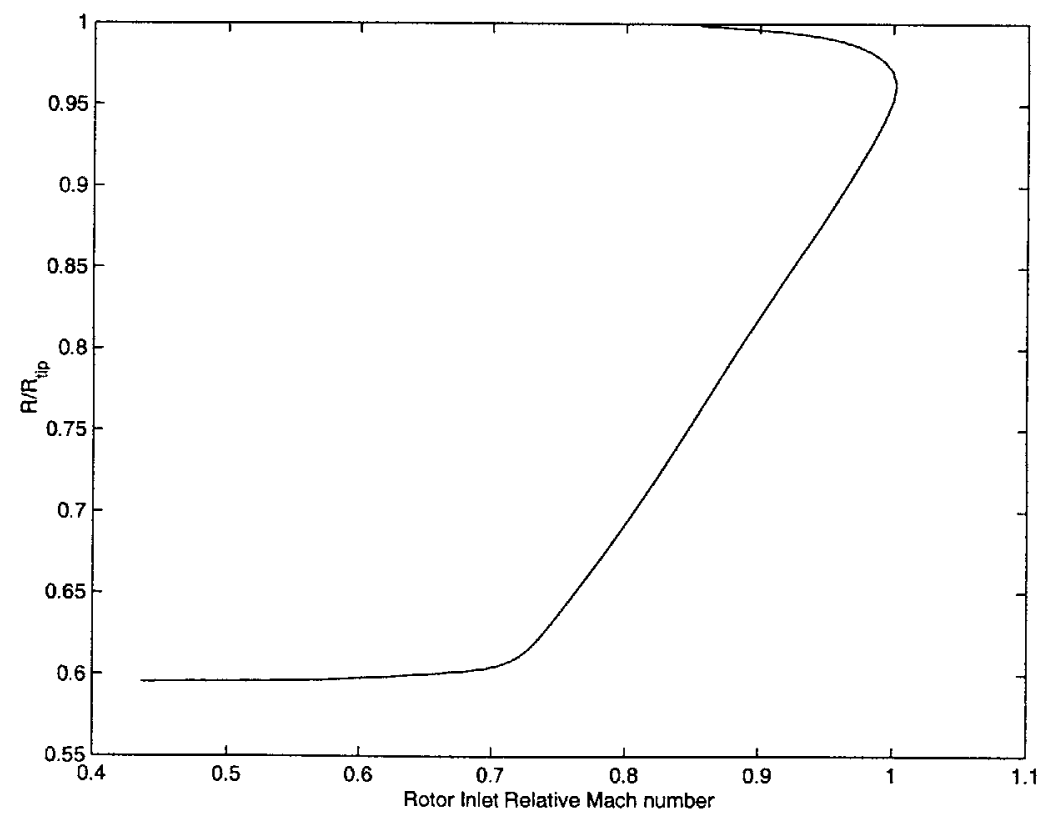

Figure 2-3: Rotor inlet relative mach number from APNASA 


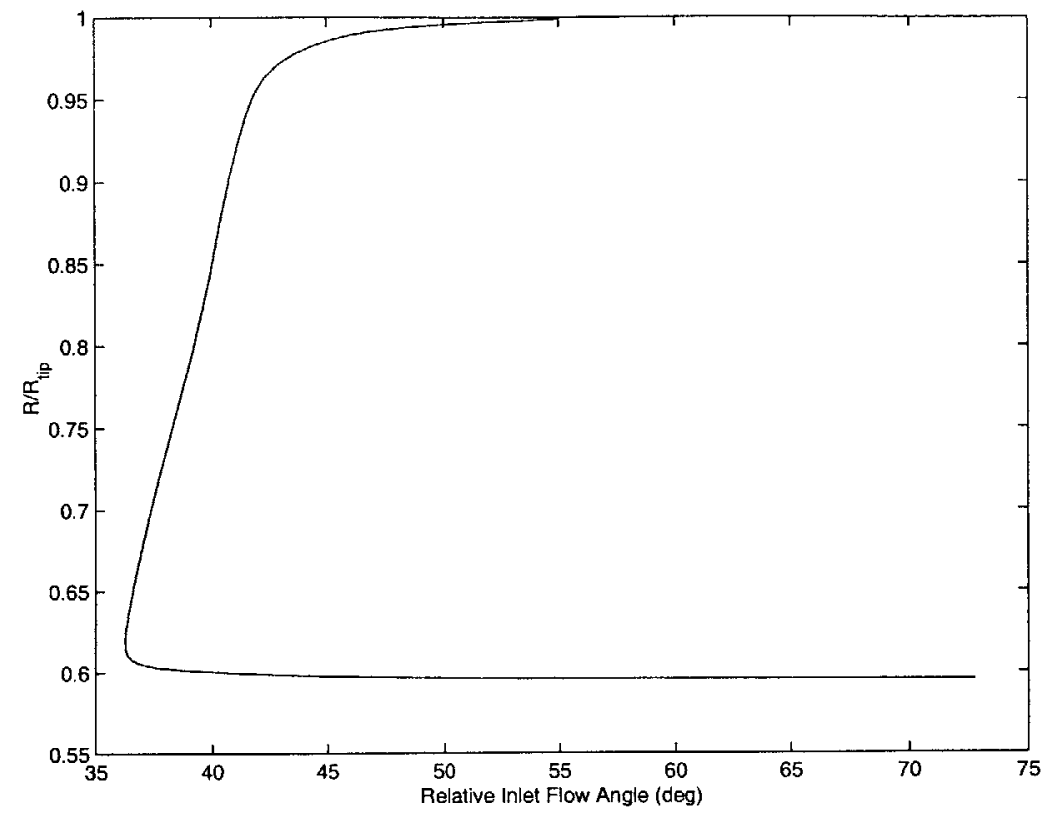

Figure 2-4: Rotor inlet relative flow angle from APNASA

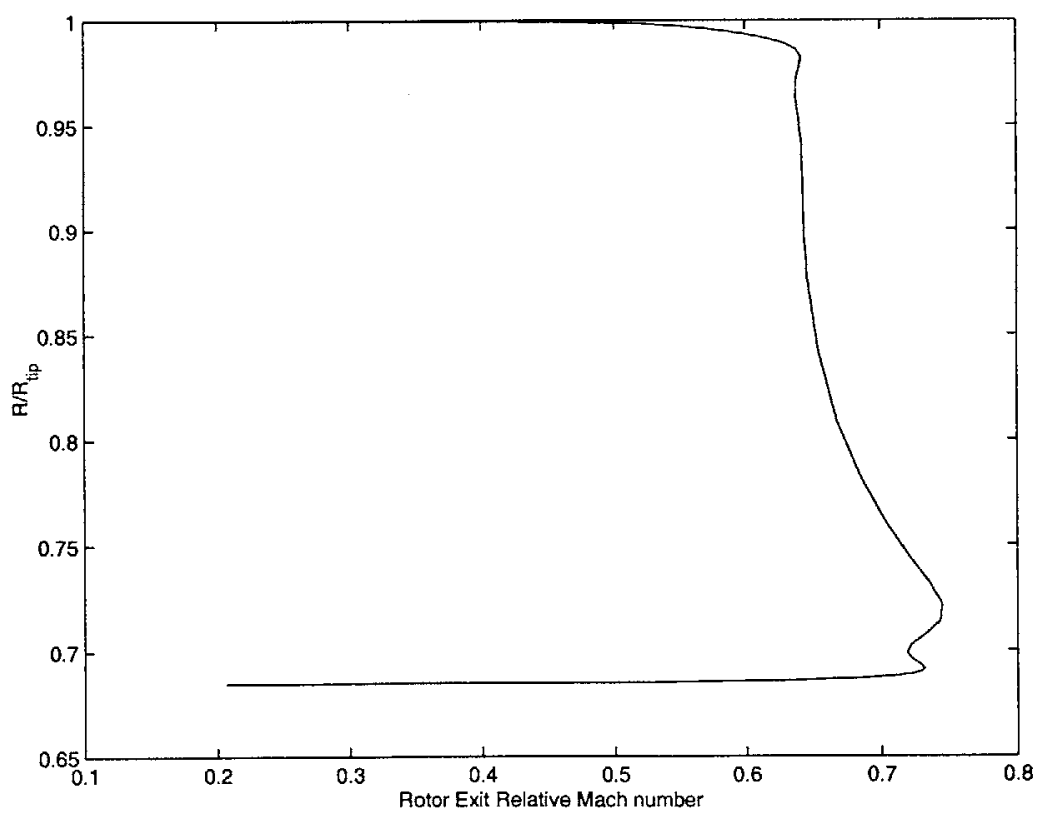

Figure 2-5: Rotor exit relative mach number from APNASA 


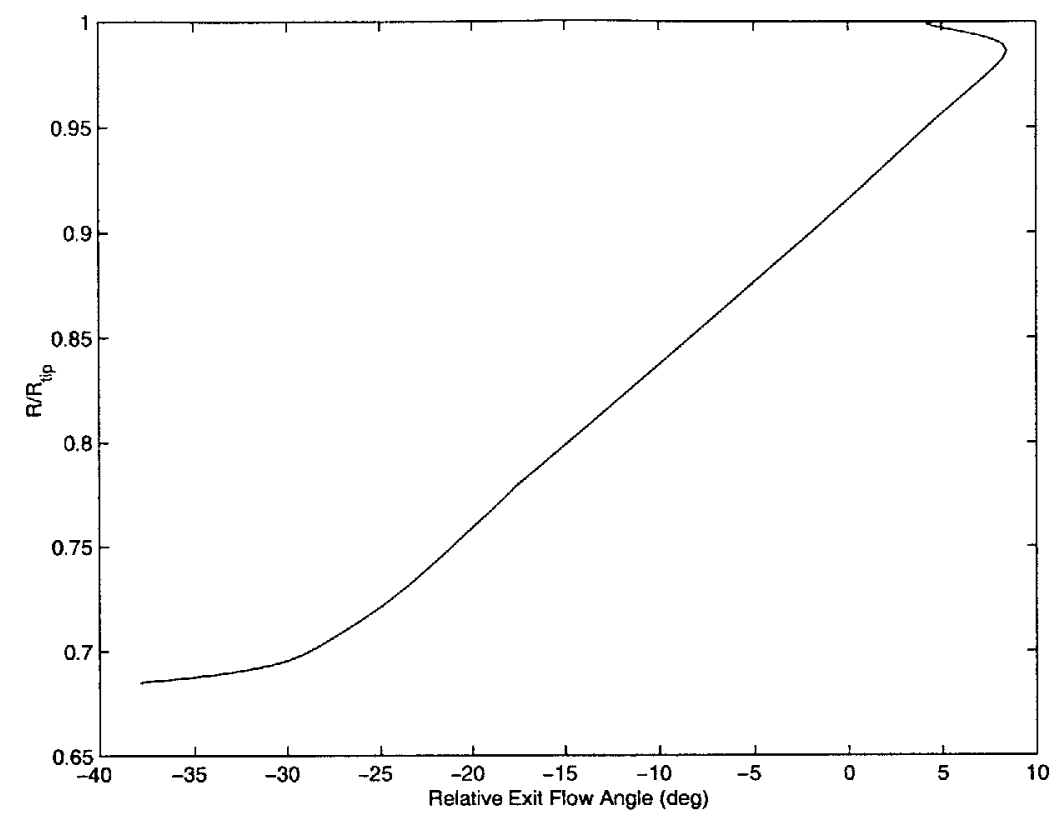

Figure 2-6: Rotor exit relative flow angle from APNASA

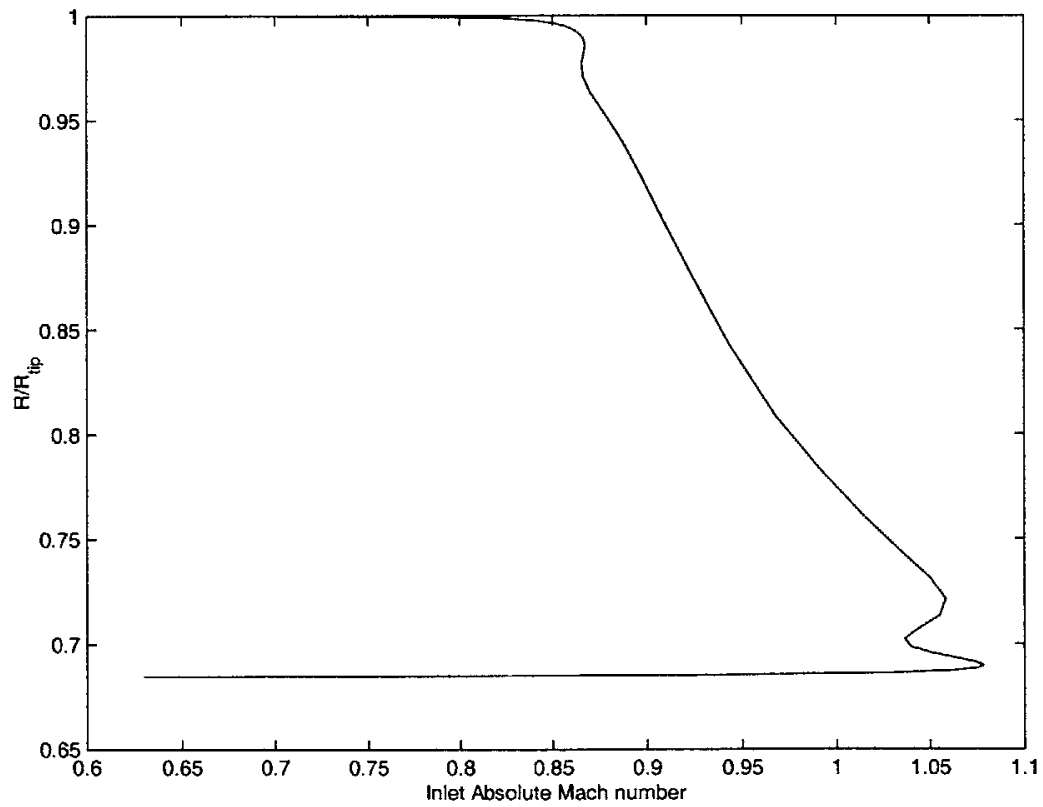

Figure 2-7: Stator inlet absolute mach number from APNASA 


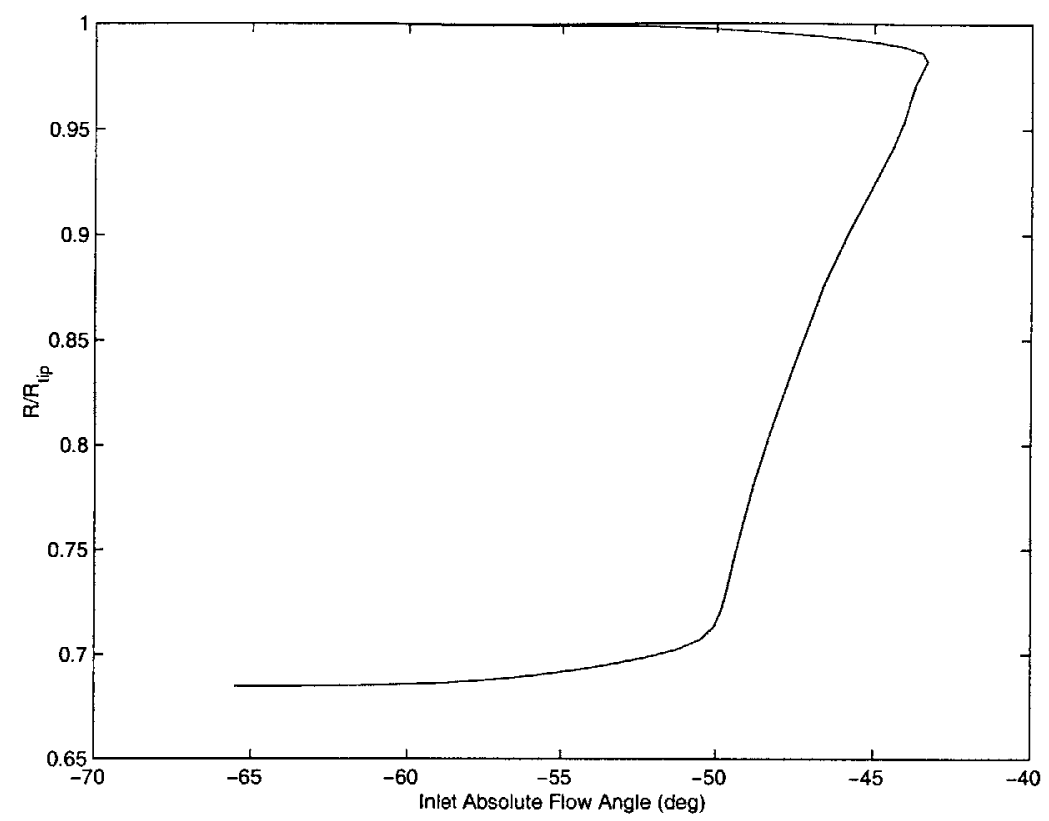

Figure 2-8: Stator inlet absolute flow angle from APNASA

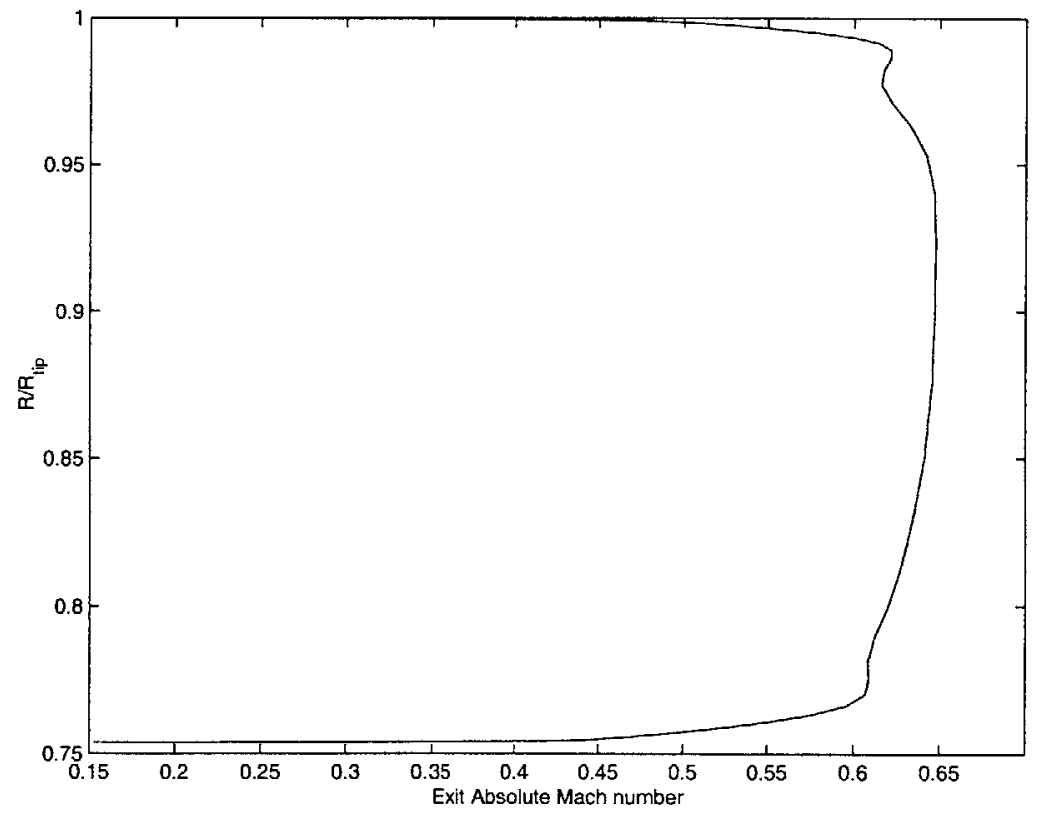

Figure 2-9: Stator exit absolute mach number from APNASA 


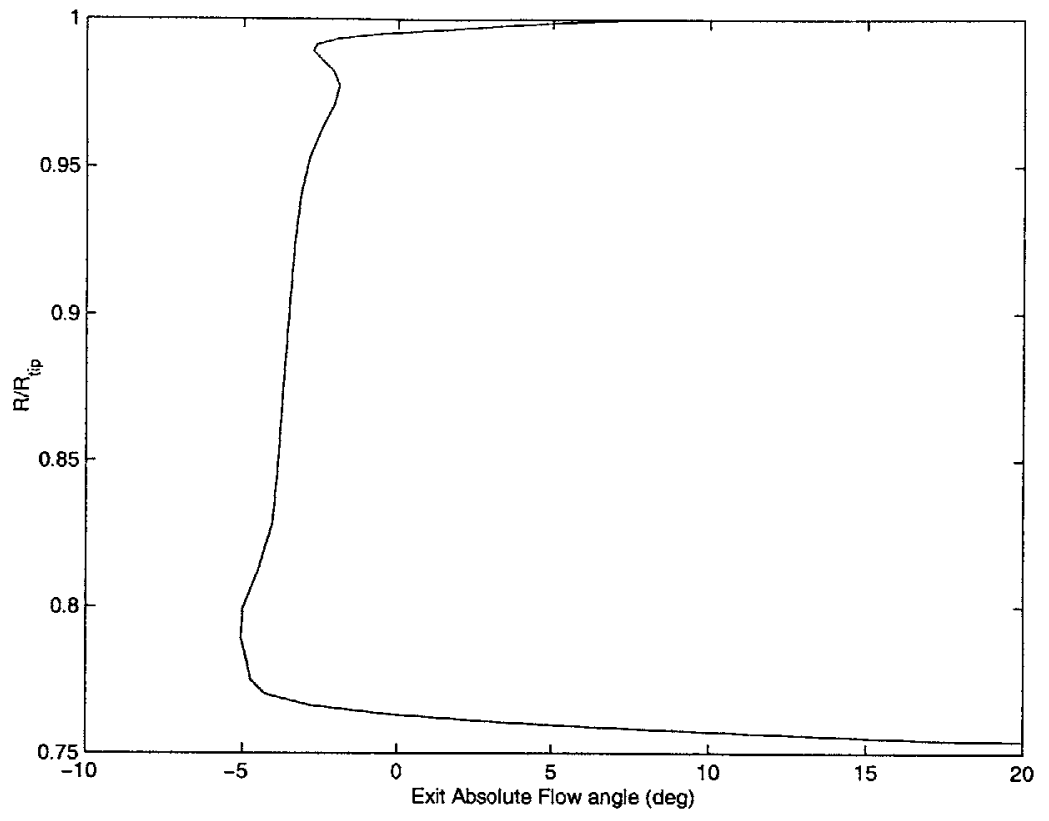

Figure 2-10: Stator exit absolute flow angle from APNASA

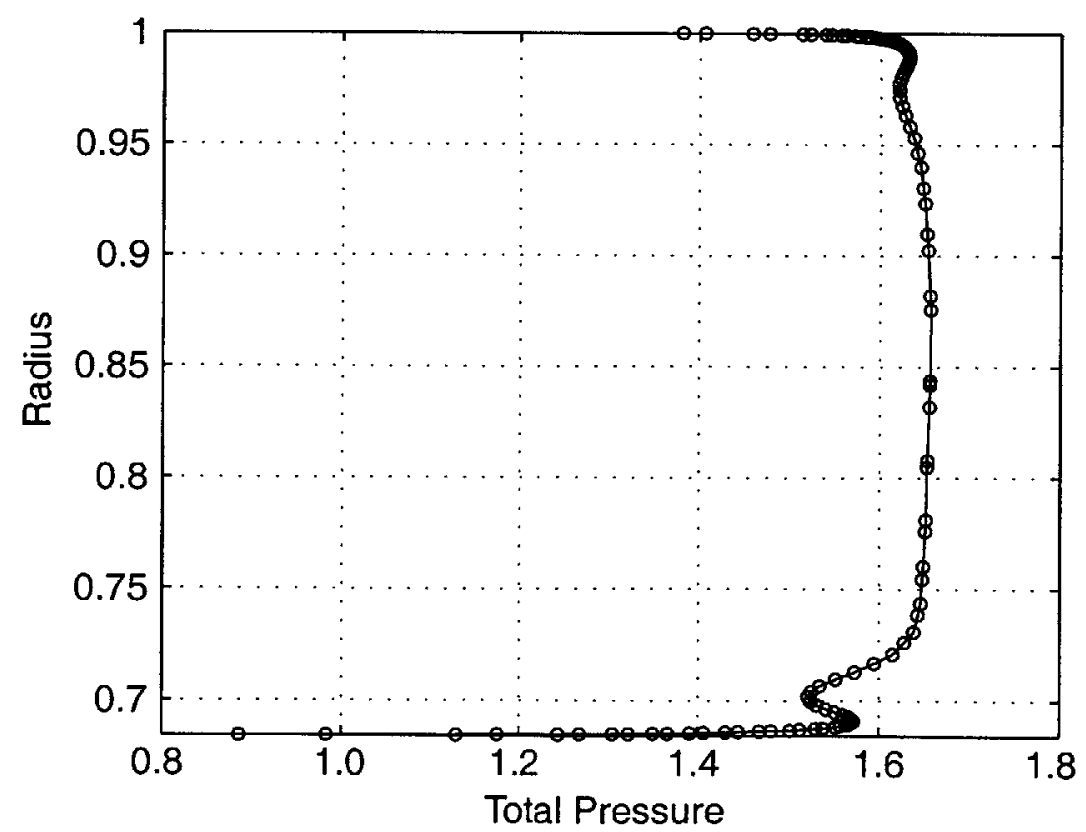

Figure 2-11: Total pressure at rotor exit from APNASA analysis 


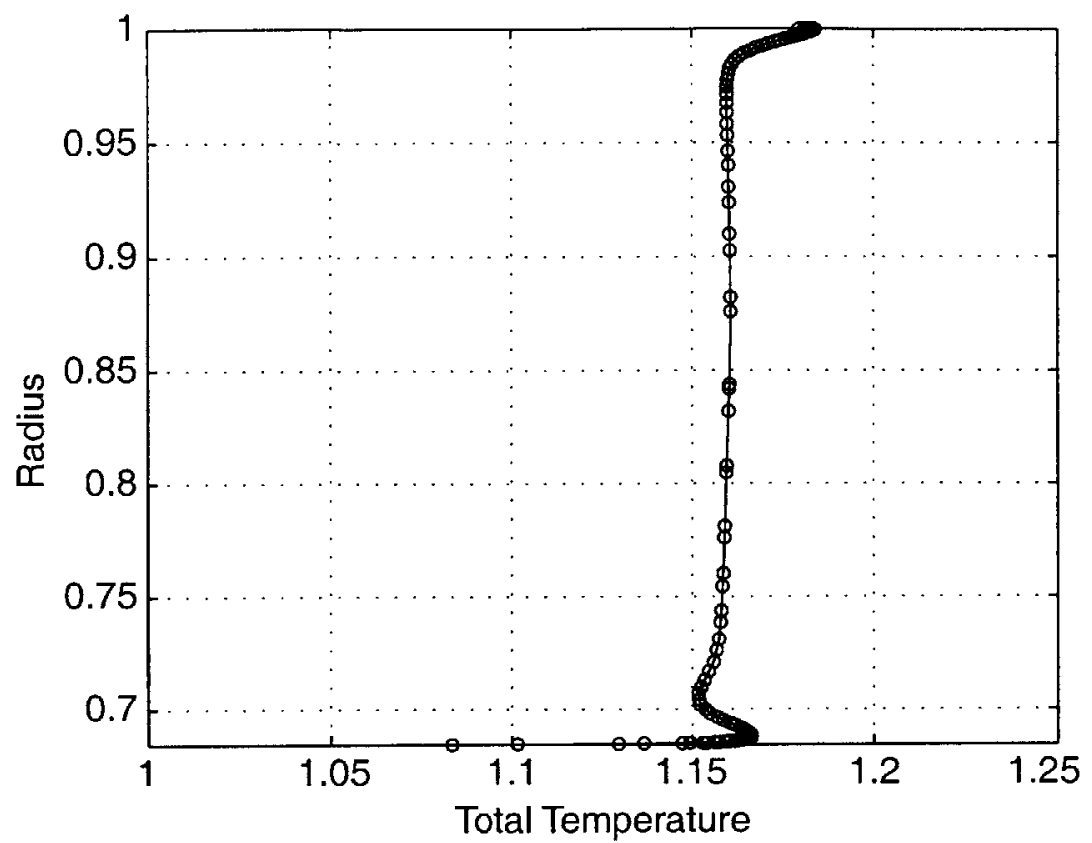

Figure 2-12: Total temperature at rotor exit from APNASA analysis

\subsubsection{APNASA Analysis}

The data shown in figures 2-11,2-12, 2-13, and 2-14 show the tangentially-averaged results of the APNASA analysis of the fan stage. The suction flow values used in the APNASA analysis are the same as those given in section 2.3.3, specifically tables 2.4 and 2.5. Figures 211 and 2-12 show the tangentially-averaged total pressure and total temperature predictions, respectively, made by the APNASA analysis for the exit of the rotor and are taken from Merchant [14]. The analysis shows a constant radial total pressure distribution for the rotor except for a small bump at the hub. The design was intended to be a free vortex design, and the total temperature profile indicates that this is the case. The slight rise in total temperature near the tip is due to the energizing of the shroud boundary layer due to the rotating shroud.

Figures 2-13 and 2-14 show the pitch-averaged total pressure and total temperature predictions made by the APNASA analysis for the exit of the stage. The analysis shows a drop in total pressure near the hub and near the tip of the stator exit. The total pressure deficit near the hub is due to high shock losses in the stator and an increase in the hub boundary layer thickness. The total pressure deficit near the tip is not as extensive radially 


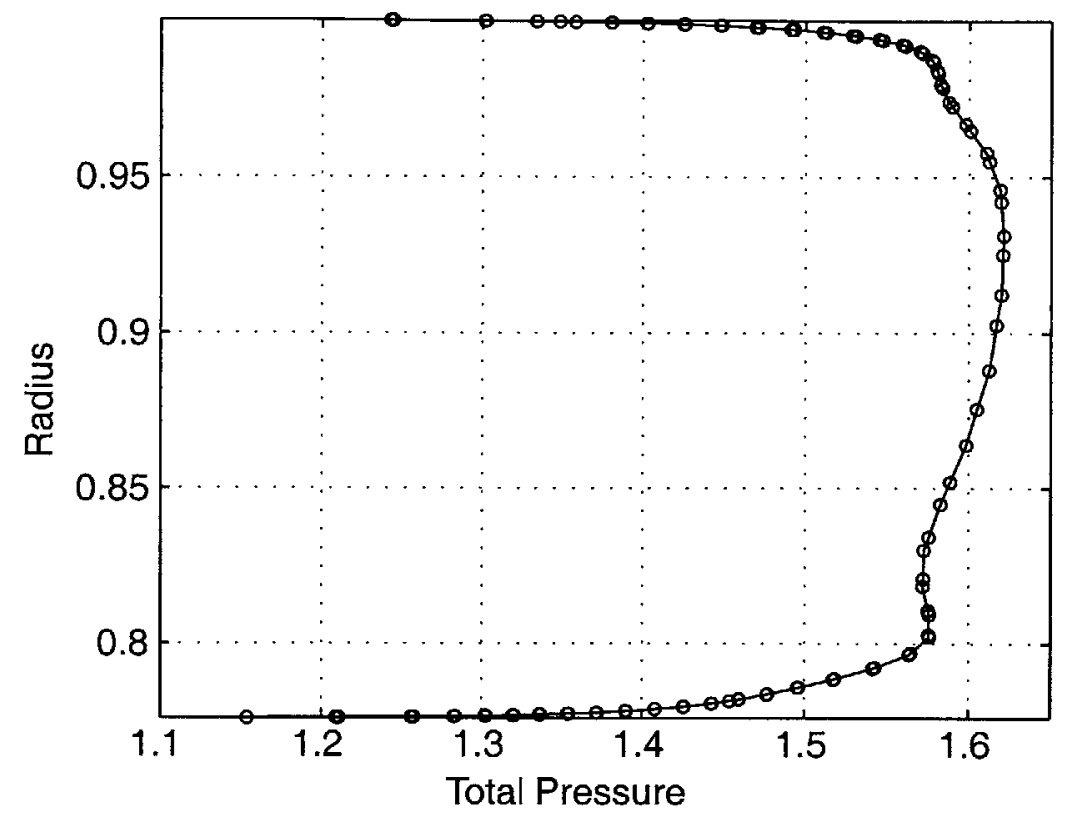

Figure 2-13: Total pressure at stator exit from APNASA analysis

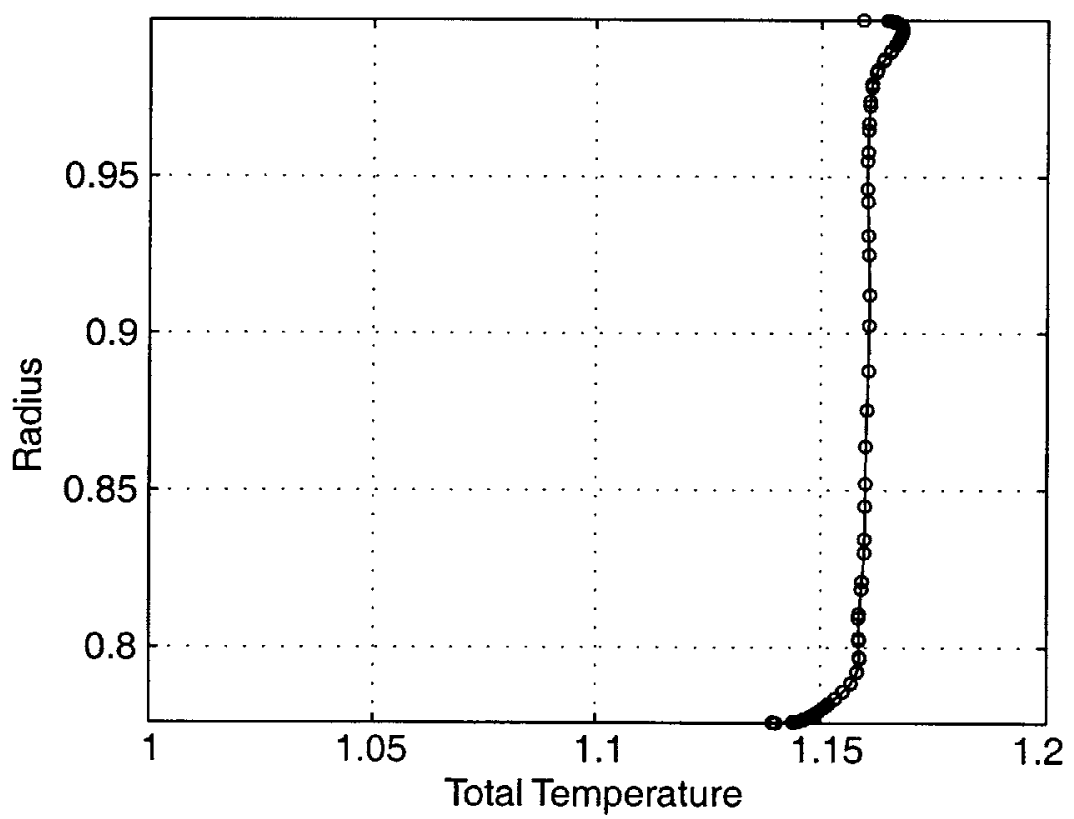

Figure 2-14: Total temperature at stator exit from APNASA analysis 


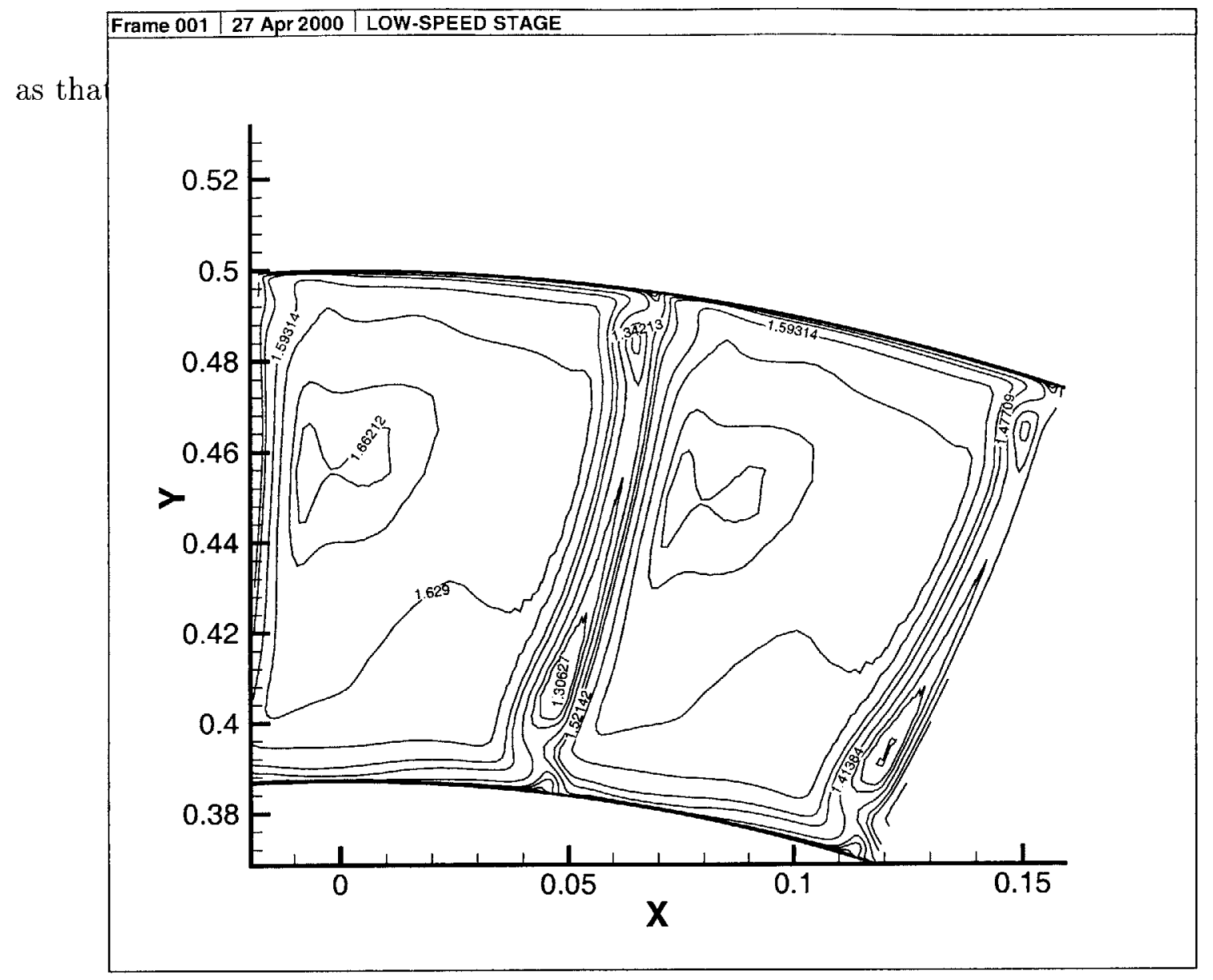

Figure 2-15: Total Pressure Contours from APNASA analysis

Figure 2-15 shows a contour plot of the calculated total pressure ratio downstream of the stator. It is important to notice the two low total pressure regions that have developed near the endwalls on the suction side of the stator blade. These two regions represent the regions of high loss produced by the stator. Reduction of these two total pressure holes could mean as much as a $3 \%$ benefit to the overall stage efficiency.

\subsubsection{Incidence Mismatch}

After the designs for both the rotor and stator had been completed in MISES, the stator was in the middle of production when the rotor and stator were first analyzed in APNASA. Due to an unaccounted-for blockage, the stator could not pass the required mass flow to allow the rotor to run at its design mass flow. Since the stator was already in construction, its design could not be changed to match the rotor. Instead, the rotor hub line was raised to 
lower the mass flow passed by the rotor at design speed so that the stator could run as close to its design mass flow as possible. With this correction the stator runs at a slightly higher incidence than its intended design. Therefore, the stator's peak efficiency mass flow does not correspond to the stage's peak efficiency mass flow, as shown by the $3.5 \%$ efficiency loss in the rotor compared to a $7.5 \%$ efficiency loss in the stator. A new stator design could increase the overall stage adiabatic efficiency to better than $92 \%$. The stator redesign is shown in Chapter 7. 


\subsection{Mechanical Design}

The mechanical approach for this project is described by Schuler in reference [19]. The outline for construction of the stage is presented as well as a procedure for removing the suction flow from the main flow path. In reference [14], Merchant's design deals with blade surface suction, but suction also has to be applied to the endwalls in order to prevent separation of the endwall boundary layers. This endwall suction scheme is presented in figure 2-16, and the suction values are shown in tables 2.4 and 2.5. The completed assembly, shown in figure 2-16, was constructed at MIT and placed in the MIT Blowdown compressor facility for testing.

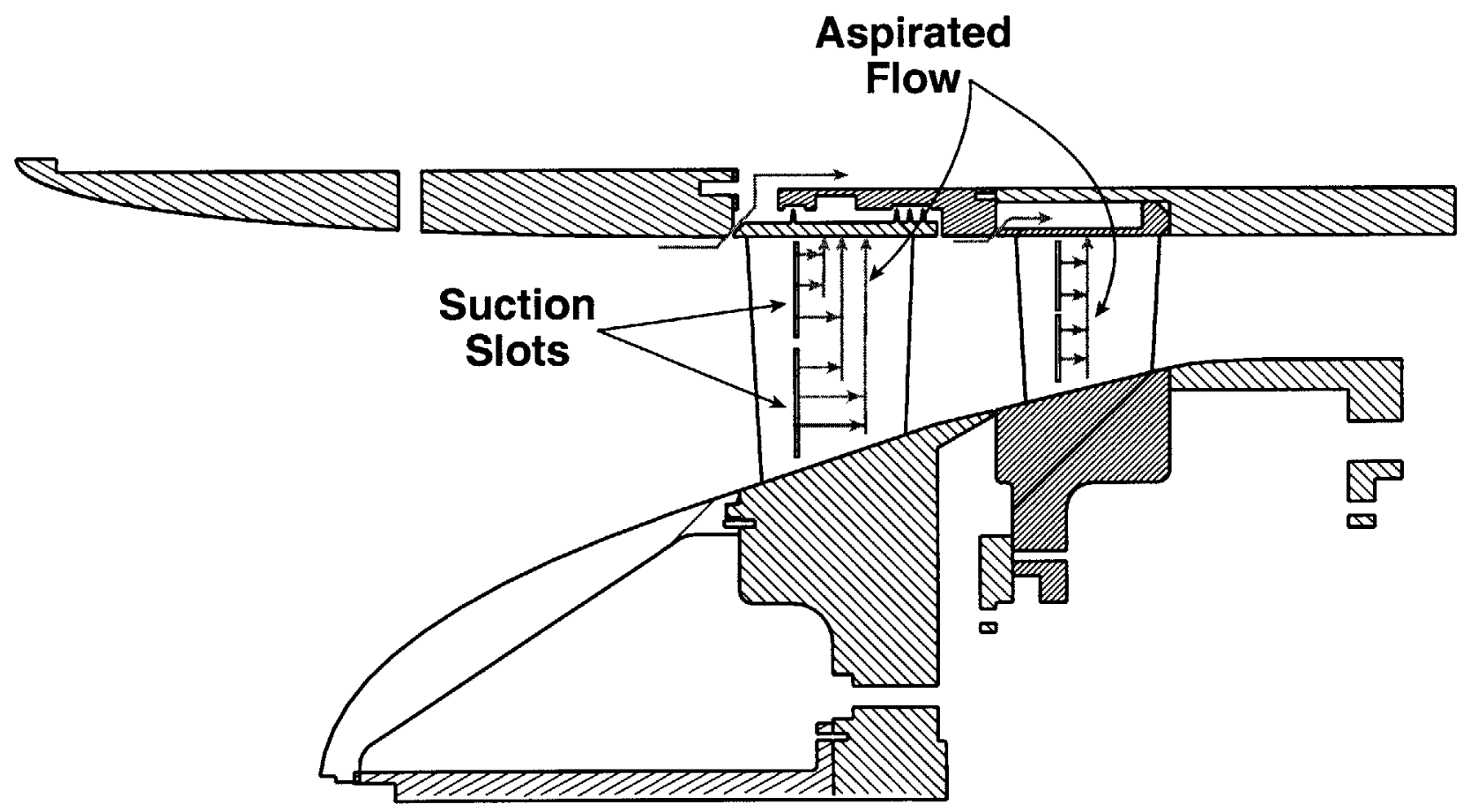

Figure 2-16: Assembly cross-section and suction scheme

\subsubsection{Stage Construction}

An integral shroud was incorporated with the design of both the rotor and stator. For the rotor, the integral shroud provides a convenient path for dealing with the blade suction flow. Reijnen [17] removed the suction flow from the root of the rotor blades and used a 
non-rotating spider with a rotating seal to dispose of the aspirated flow. Taking the suction flow radially inward requires a separate dump tank because the pressure in the suction flow dump tank must be much lower than the main dump tank to allow an appreciable test time. The integral shroud allows the flow to be taken radially outward. Figure 2-16 shows a cross-section of the fan stage as well as the suction removal system. The increase of pressure radially due to rotation produces higher pressures at the metering orifices in the shroud and allows the flow to be dumped into the same dump tank as the main flow.

Another significant reason for use of an integral shroud was the elimination of the tip clearance flow. Because the aspirated stage has such a high blade loading, especially at the rotor tip, the tip clearance flow could become the dominating fluid mechanical process within the stage. Therefore, the integral shroud design was adopted to eliminate this uncertainty and to provide a computationally simpler flow domain.

\subsubsection{Suction Removal System}

Figure 2-16 shows the suction scheme presented by Schuler [19]. The shrouded rotor allows the suction flow to be taken out through the tip of the rotor and stator blades. The flow then travels down axial channels milled out of the casing and is exhausted into the dump tank. Figure 2-17 shows the rotor blade suction surface both with and without the cover plate. The blade without the cover plate shows the three suction channels used to carry the flow from the suction slot to the shroud. The blade with the cover plate shows the position of the suction slot during testing. The suction flow is metered by the choke holes drilled in the shroud sections of both the rotor and stator and shown in figure 2-18.

Only the suction flow within each of the three casing channels can be measured. A pair of static and total pressure probes are placed at the same axial position within each suction channel. Measurements of the first suction channel quantify the casing suction flow ahead of the rotor. The second suction channel carries the rotor blade suction and the rotor shroud suction as well as the labyrinth seal leakage flow from the rear of the rotor. The stator blade suction, stator shroud suction, and stator leakage flow are all carried by the third

suction channel. The total and static pressure probes along with an estimate of the total 


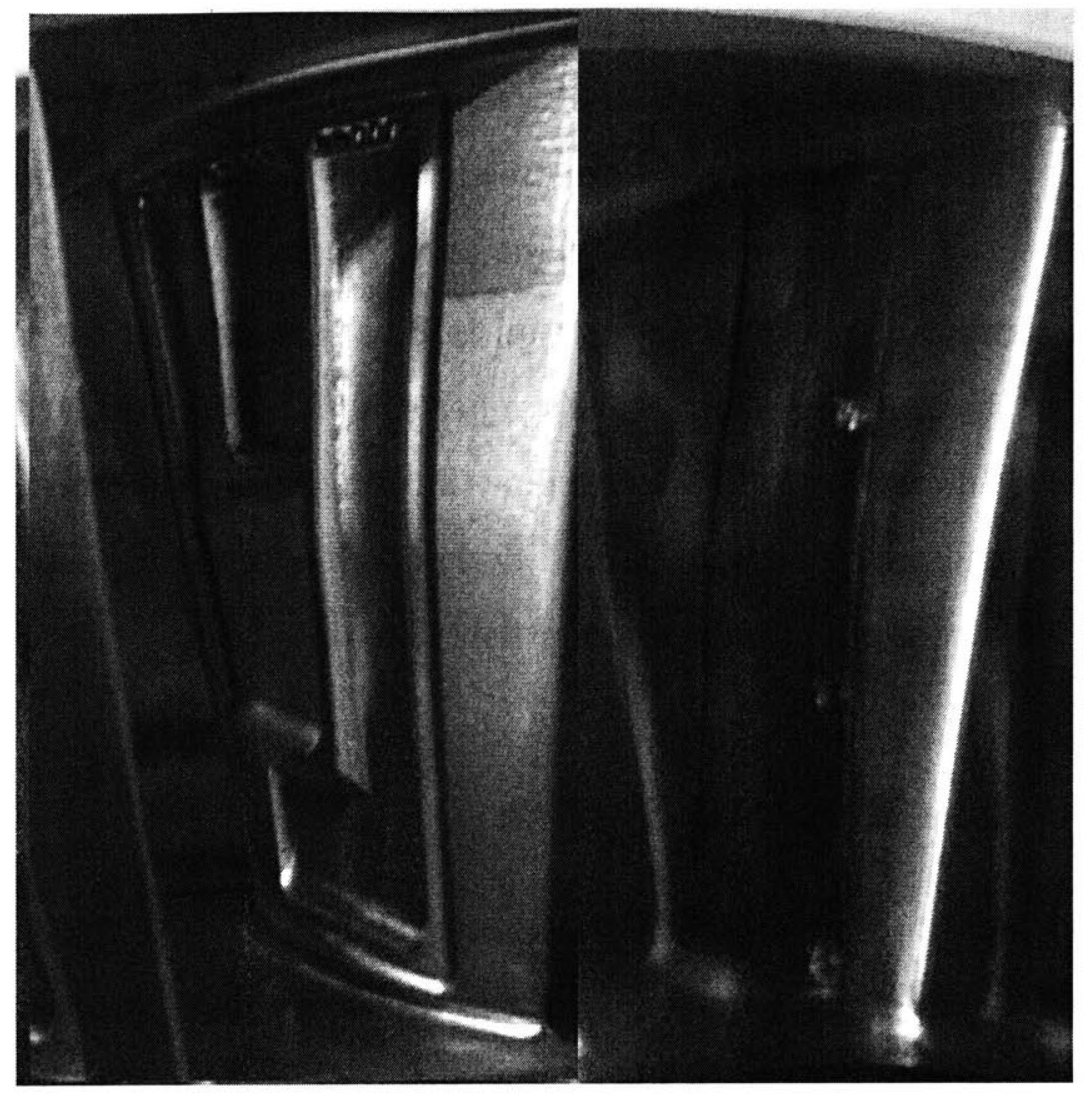

Figure 2-17: Rotor blade with and without cover plate 


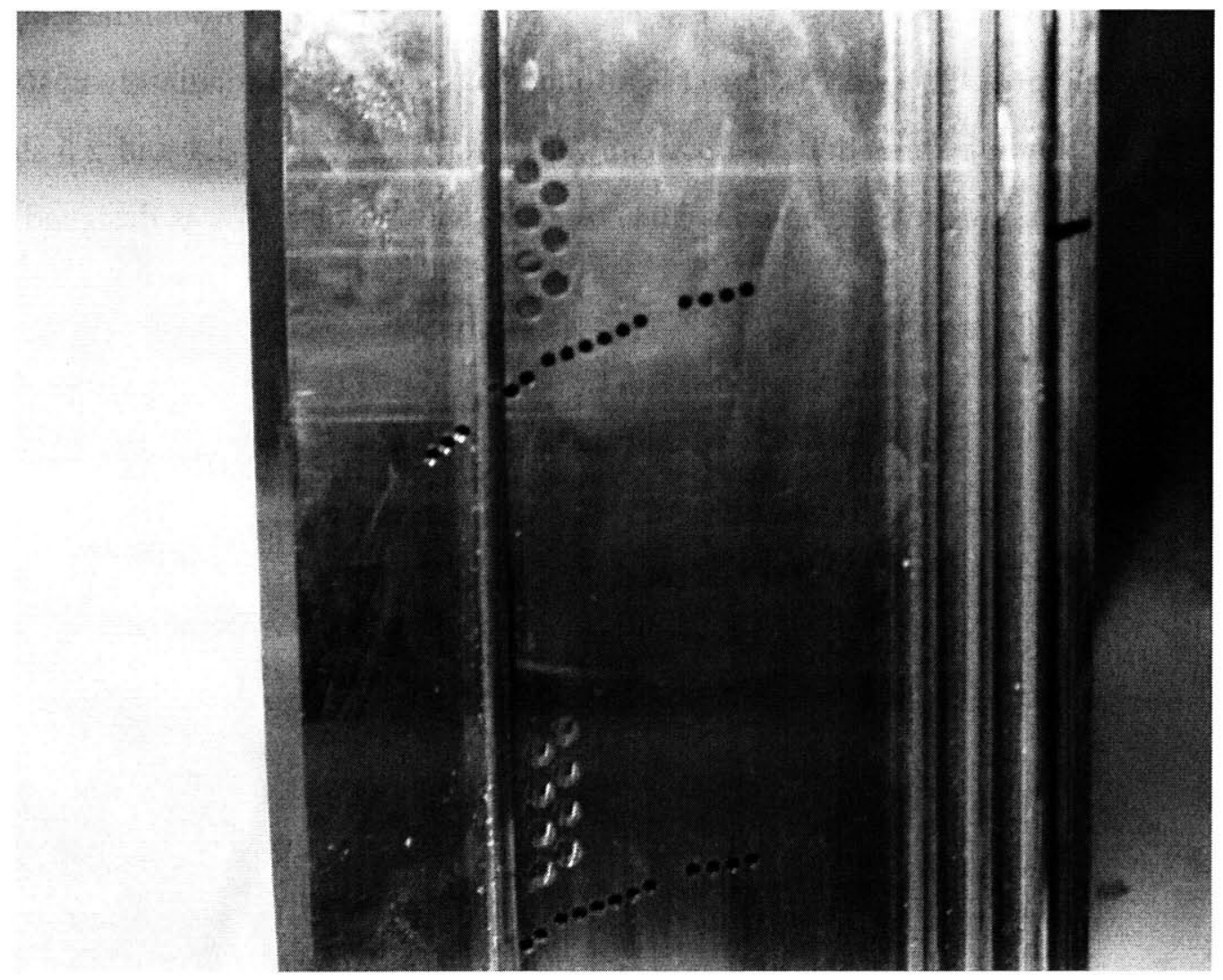

Figure 2-18: Rotor Shroud with Blade Suction Choke Holes 
temperature within the channel quantify each channel's suction flow.

\subsubsection{Total Suction Requirement}

The design of the low speed aspirated stage required a blade suction of $0.5 \%$ per blade row on the blade surfaces. In this low speed design, the rotor tip blade section and the stator hub blade section are the most highly loaded sections within the stage with diffusion factors of 0.56 and 0.54 respectively. These blade sections require thin endwall boundary layers for maximum performance. Therefore, suction is applied to the casing immediately upstream of the rotor and on the hub immediately upstream of the stator. Tables 2.4 and 2.5 show the suction amounts as a percent of the inlet flow. A total suction of $4.73 \%$ is designed for the stage.

Table 2.4: Rotor Suction Requirements

\begin{tabular}{||l|l|}
\hline Blade Suction & $0.50 \%$ \\
Casing Suction & $1.29 \%$ \\
Blade to Blade Shroud Suction & $0.45 \%$ \\
\hline Total Rotor Suction & $2.24 \%$ \\
\hline
\end{tabular}

Table 2.5: Stator Suction Requirements

\begin{tabular}{||l|l|}
\hline Blade Suction & $0.50 \%$ \\
Casing Suction & $1.04 \%$ \\
Hub Suction & $0.50 \%$ \\
Blade to Blade Shroud Suction & $0.45 \%$ \\
\hline Total Stator Suction & $2.49 \%$ \\
\hline
\end{tabular}

The suction amounts presented in tables 2.4 and 2.5 accurately reflect the scheme implemented for the low speed experiments but do not represent the suction scheme implemented in the APNASA analysis. The blade to blade shroud suction on both the rotor and stator were not used in the final APNASA analysis. Therefore, the total suction for this stage, according to the APNASA analysis, is $3.7 \%$, which is lower than the $4.7 \%$ suction in the experiment. 


\subsection{Summary}

The aerodynamic design of the low speed experiment uses MISES as the main design tool to produce the blade shapes. The inverse design mode allows the designer to input the desired blade pressure distribution and outputs the blade geometry. MISES's limitations are that it can not predict the strong radial flows that are associated with the low momentum fluid near the blade surfaces and does not resolve the endwall boundary layers. The APNASA analysis tool is used to resolve these flows and to predict the full performance of the stage, although, as will be shown later, it does not embrace important effects due to unsteadiness in blade coordinates. If design changes are required, the results of the analysis can be fed back into the MISES design tool to change the blade shapes.

The APNASA analysis shows the spanwise distribution of total pressure and total temperature at the exits of both the rotor and stator. The results of the APNASA analysis are a design total pressure ratio of 1.59 at an adiabatic efficiency of $89 \%$ while the MISES results were a total pressure ratio of 1.63 at an adiabatic efficiency of $94 \%$. The endwall boundary layers and secondary flows, along with a slightly off-design stator incidence angle, caused a decrease in adiabatic efficiency.

The mechanical design and suction removal systems are designed to take full advantage of the blowdown compressor facility. The low dump tank pressure is used to drive the suction flows which exhaust into the dump tank along with the main flow. Choke holes machined into the rotor and stator shrouds meter the suction flows so that the design suction levels are reached. It is important to note that the APNASA analysis has predicted the required total suction for the stage to be approximately two thirds lower than the suction level implemented in the experiment. This lower suction level, if verified, would make the low speed stage much more attractive as a high efficiency fan stage for civil aircraft applications. 


\section{Chapter 3}

\section{Experimental Setup and Data}

\section{Acquisition}

\subsection{Introduction}

This chapter focuses on the experimental test facility and data acquisition system used to gather the data on the aspirated fan stage. First, an overview of the basic operation of the test facility is given including an overview of the test procedure. Next, the different types of instrumentation are described including the static wall pressure transducers, the 4-way probe, the total and static probe, and the optical encoder. Then the details of the data collection system are presented including the number of data channels and sampling rates. Finally, the error analysis of the instrumentation shows the confidence bounds on the data collected.

\subsection{Testing Facility}

The fan stage was tested in the MIT Blowdown compressor. The design, construction, and primary testing procedures are described by Kerrebrock in reference [5]. Since the inception of the blowdown compressor, several validations of the test procedure have been performed, and the explosively-cut aluminum diaphragm has been replaced by a fast-acting valve that 
allows multiple tests to be performed daily. A detailed description of the fast-acting valve can be found in reference [28] by Ziminsky. The facility is instrumented with many high frequency response pressure transducers to capture the flow field within the test section and the necessary pressures within the supply and dump tanks.

\subsubsection{Blowdown Compressor Operation}

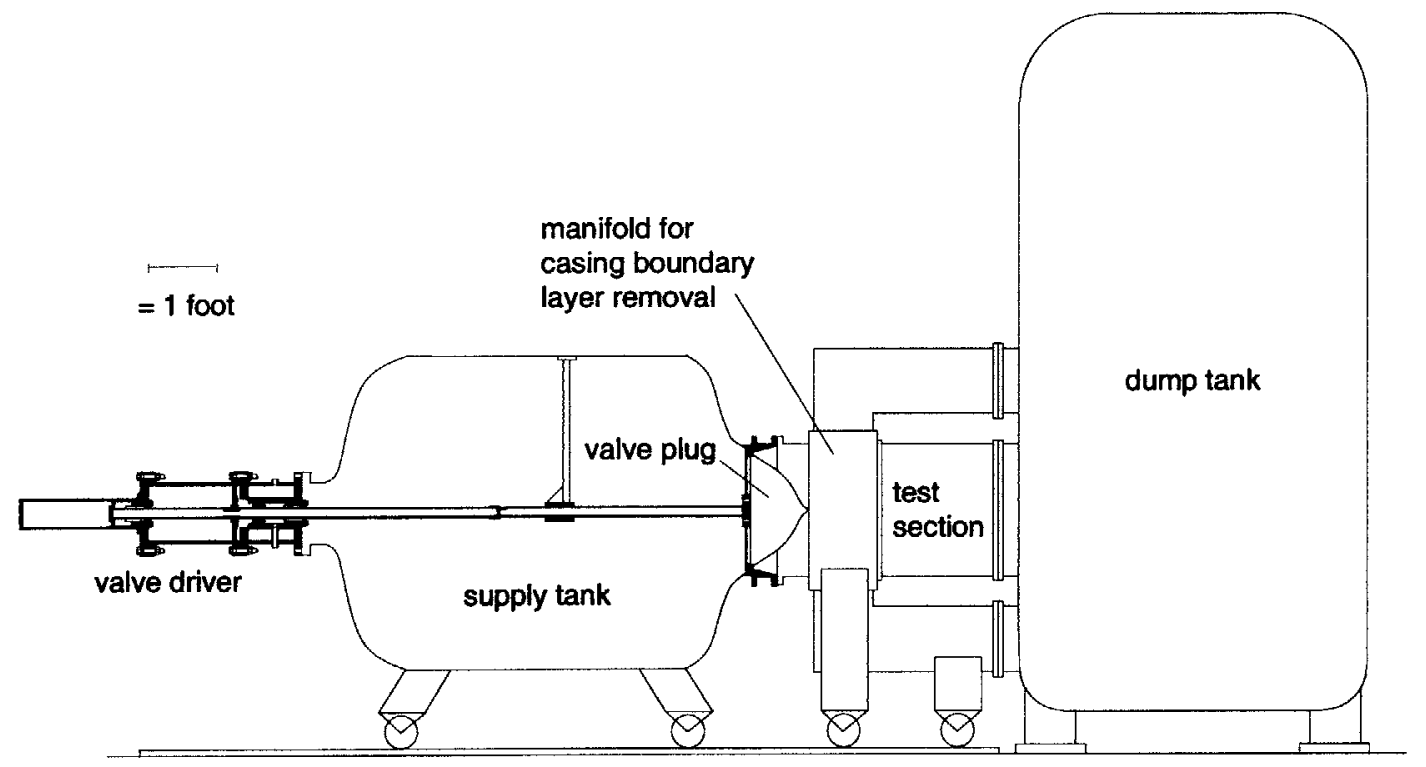

Figure 3-1: Blowdown Compressor Schematic

Figure 3-1 shows a cross-section of the test machine. A blowdown experiment begins with the evacuation of the complete facility. Then the supply tank is isolated from the rest of the facility by the fast-acting valve plug. The supply tank is then pressurized with a mixture of argon and carbon dioxide to a pressure of $609 \mathrm{mmHg}(.80 \mathrm{~atm})$. After the supply tank is pressurized, the rotor is spun up to speed in the evacuated space of the test section. Once the design speed is reached and surpassed, the motor is turned off, and the rotor is allowed to rotate freely in the test section. When the rotor coasts back down to the design speed, the fast-acting valve releases the gas from the supply tank allowing it to flow into the test section. The spinning rotor imparts some of its kinetic energy into the incoming flow thus producing a pressure ratio across the stage. The mass flow of the experiment is set by the 
choke plate located just downstream of the stator and upstream of the dump tank. The test is completed when the dump tank pressure rises to a high enough value so as to unchoke the choke plate or the suction channels. The total flow time is approximately 400 milliseconds from valve release to pressure equilibrium within the facility. After the startup transients, the usable test time is approximately 75 milliseconds with the remaining test time associated with equilibrating the pressure between the supply tank, dump tank, and test section.

The supply tank is filled with a mixture of argon and carbon dioxide such that $\gamma$, the ratio of specific heats, is 1.4. Due to the heavier gases used, the mixture has a higher molecular weight than air. With this mixture, the speed of sound during the test is approximately $80 \%$ of the speed of sound in air. Therefore, for a constant rotor blade Mach number, the rotational speed of the rotor during the test is less than the same test done in air, and the

lower speed translates to lower stress levels in the rotor. A more detailed description of the the blowdown compressor firing system can be found in reference [17].

The boundary layer manifolds remove approximately $10 \%$ of the flow from the supply tank in order to assure that a uniform flow enters the test section.

\subsubsection{Data Collection System}

The most important part of the data collection system is the 4-way probe shown in figure 3-2. The probe contains four high frequency Kulite transducers. Transducer $1\left(P_{1}\right)$ is placed so as to directly face the oncoming flow and closely measures the total pressure of the flow. Transducers 2 and 3 are placed nearer the shank and 45 degrees on either side of $P_{1}$. Given a suitable calibration, these three transducers are responsible for determining the total pressure, static pressure, and tangential flow angle. Transducer 4 is mounted on a 45 degree wedge cut from the tip of the probe and, with calibration, provides a measurement of the radial flow angle. In reference [17], Reijnen describes the calibration process used for the 4-way probe data reduction.

High frequency pressure transducers are also placed within the casing of the stage and measure the casing static pressures upstream and downstream of the stage as well as between the rotor and stator. The downstream casing pressure, along with the exit Mach number set 
by the choke plate area ratio, gives an estimate of the stage total pressure ratio and can be used to make some predictions of overall stage performance.

An optical encoder with 400 lines per revolution is used to measure the speed of the rotor. The encoder also has the option of producing a once per revolution pulse so that the test is always triggered with the rotor in the same position.

\subsection{Data Acquisition}

The data aquisition system used in the Blowdown Compressor facility consists of high frequency Kulite transducers, an analog pressure gauge, an optical encoder, and one temperature sensor. The high frequency Kulite transducers are mounted in one of three places: the 4-way probe, the stage casing, or the total and static probes. The analog pressure gauge is located in the supply tank and gives the initial supply tank pressure at the beginning of a test. The frequency of the square wave from the optical encoder gives the shaft rotational speed. The temperature sensor is located in the supply tank and gives the initial temperature of the supply tank gas. Some details of specific components of the data acquisition system are presented in the following sections.

\subsubsection{Wall Static Transducers}

The wall static transducers are placed in three casing locations within the aspirated stage: $\frac{1}{3}$ rotor chord upstream of the rotor, $\frac{1}{3}$ rotor chord downstream of the rotor, and $\frac{1}{2}$ stator chord downstream of the stator. The wall static transducers provide basic data on the global performance of the fan stage. Specifically, important parameters like rotor inlet Mach number, stage total pressure ratio, and tip rotor reaction can be estimated from wall static transducers and some basic assumptions based on the 3 -D viscous analysis results.

$$
\frac{P_{\text {supply }}}{P_{\text {wall }}}=\left(1+\frac{\gamma-1}{2} M_{\text {rotor }}^{2}\right)^{\frac{\gamma}{\gamma-1}}
$$

The rotor inlet Mach number is determined using equation (3.1), where $P_{\text {supply }}$ is the 
supply tank pressure, $P_{\text {wall }}$ is the wall static pressure, and $M_{\text {rotor }}$ is the rotor inlet Mach number. The static pressure is only measured at the casing wall, therefore the radial pressure gradient must be zero for this to be an accurate estimate of the rotor inlet Mach number. The APNASA analysis shows that the static pressure at the rotor face is very nearly constant in the radial direction due to the fact that there are no inlet guide vanes (IGV's) and, hence, no swirl entering the stage.

The stage inlet mass flow is estimated from equation (3.2) using standard day conditions $\left(P_{0}=1.013 * 10^{5} \mathrm{~Pa}\right.$ and $\left.T_{0}=300 \mathrm{~K}\right)$, the rotor inlet Mach number, and the cross-sectional area at the probe's axial location, $A_{\text {probe }}$.

$$
m=P_{0} \sqrt{\frac{\gamma}{R T_{0}}} M_{\text {rotor }}\left(1+\frac{\gamma-1}{2} M_{\text {rotor }}^{2}\right)^{-\frac{\gamma+1}{2(\gamma-1)}} A_{\text {probe }}
$$

\subsubsection{Optical Encoder}

The optical encoder consists of a glass disk, an LED, and two photo electrodes. One ring on the disk contains a single line which signals the rotational position of the rotor. A second ring contains 400 equally-spaced lines which enables a finer resolution of the rotor speed. The encoder outputs three basic signals: a once per revolution pulse called I, a 400 pulses per revolution signal called $\mathrm{A}$, and another 400 pulses per revolution signal but with a 90 phase lag from A, called B. The I signal and A signal are captured by the data acquisition system and used to determine the shaft speed.

\subsubsection{Total and Static Probes}

The total and static probe was built to measure the mass flow through the suction channels. By measuring the total and static pressure at the same point within the suction channel, the channel Mach number is calculated and can be used along with the measured total pressure and estimated total temperature to get the suction flow.

In order to calculate the mass flow in the suction channel, the total temperature must be estimated. For the suction channel upstream of the rotor, the total temperature is just 
the total temperature of the supply tank. For the suction channel that carries the blade aspiration from the rotor, the total energy in the suction flow is the sum of the initial internal energy from the supply tank and the work done on the flow by the rotating blade row. Since the flow comes out radially in the coordinate system of the rotor, the specific work input by the rotor is $\frac{1}{2}$ times the rotor tip speed squared. Equation (3.3) expresses this relationship, where $\tau_{\text {channel }}$ is the ratio of channel total temperature to supply tank total temperature and $M_{t}$ is the tip blade Mach number based on the supply tank total temperature.

$$
\tau_{\text {channel }}=1+\frac{\gamma-1}{2} M_{t}^{2}
$$

For the suction channel that carries the stator suction flow, the total temperature is equal to the mass-averaged total temperature entering the stator.

Once the total pressure, total temperature, Mach number, and suction channel crosssectional area are known, the suction channel mass flow is calculated and compared with design values. The results are presented in the next chapter. Appendix A shows the mechanical drawing of the probe.

\subsubsection{4-way Probe}

The 4-way probe is the most important data gathering system used in the experiment. The probe allows simultaneous measurement of total and static pressure, radial flow angle, and tangential flow angle. To achieve this goal, Kulite high frequency response transducers are mounted on an elliptically shaped cylinder that is inserted directly into the flow. One transducer $\left(P_{1}\right)$ directly faces the incoming flow and measures the pressure very close to the total pressure. The second and third transducers $\left(P_{2}\right.$ and $\left.P_{3}\right)$ are mounted aside and 45 degrees up on both sides of the first transducer. The fourth diaphragm $\left(P_{4}\right)$ is mounted on a 45 degree wedge cut from the bottom of the elliptical cylinder. Figure 3-2 shows the drawing of the 4-way probe with the diaphragms numbered and positioned. The figure is taken from Gertz in reference [4]. Appendix B shows the mechanical drawings of the window 
and sliding plate attached to the blowdown compressor to provide the 4-way probe access to the flowfield.

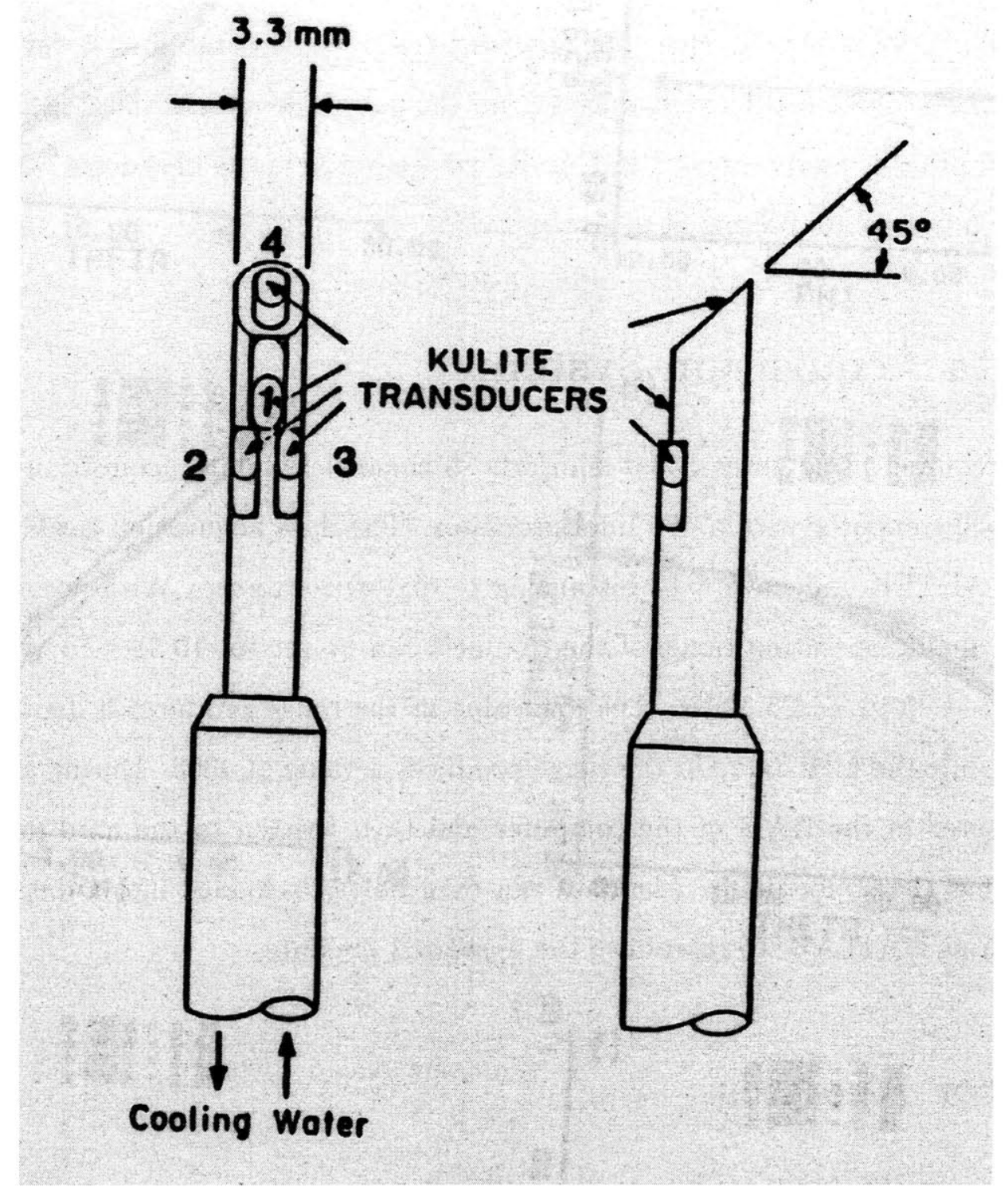

Figure 3-2: Schematic of the 4-way probe

The four transducers measure four pressures which can be converted into total pressure, static pressure, tangential flow angle, and radial flow angle with a suitable calibration. $P_{1}$ and $P_{4}$ provide the radial angle information. $P_{1}, P_{2}$, and $P_{3}$ provide total and static pressure information. $P_{2}$ and $P_{3}$ provide the tangential angle. The total Mach number is deduced from the total and static pressure, and the Mach number components are determined from 
the total Mach number and the flow angles.

Two different 4-way probes were used in the experiment. The first 4-way probe was used entirely on the rotor exit flowfield and used the calibration scheme and calibration data presented by Reijnen [17]. Due to a damaged transducer on the first 4-way probe, a second 4-way probe of identical layout was used for the data collection at the stage exit. The calibration data that is used for this 4-way probe was gathered in the blowdown compressor. The calibration figures are presented in Appendix C.

\subsection{Data Acquisition System}

The data is acquired using three 333.0 kiloHertz, 8 channel PC data acquisition cards installed in a Dell computer with a 486 Intel processor. The data acquisition cards are manufactured by ADTEK and have a 12 bit analog to digital converter. With accompanying

software, the analog operating range of the channels can be set to -10 to +10 Volts, -5 to +5 Volts, and -1.25 to +1.25 Volts. The low value in the range produces a digital output reading of 0 while the high value in the range produces a value of 4095 . During a test run, the data is stored in the RAM of the computer and then written to the hard drive when the program has the opportunity. The data can then be easily loaded into a data analysis program, such as MATLAB, to reproduce the measured pressures.

\subsection{Error Analysis}

The data taken by the 4-way probe and the wall static transducers contain noise, and this noise contributes to errors in the data reduction scheme. Reijnen [17] states that for a flow Mach number of 0.7 and the probe lined up with the mean flow, $\pm 1 \%$ noise leads to the following errors: $d \theta= \pm 1 \mathrm{deg}, d \phi= \pm 2 \mathrm{deg}, d P_{t}=0$ to $1 \%$, and $d P_{s}=-2.8 \%$ to $1.2 \%$, where $\theta$ is the tangential angle, $\phi$ is the radial angle, $P_{t}$ is the total pressure, and $P_{s}$ is the static pressure.

In addition to the pressure and velocity measurements, the efficiency of the stage needs to be calculated. This adiabatic efficiency is a function of the total pressure measured by the 
4-way probe and the total temperature, calculated from the Euler turbine equation, shown as equation (3.4), where $\tau_{c}$ is the compressor total temperature ratio, $M_{t}$ is the rotor tip blade Mach number, $M_{\tan }$ is the exit tangential Mach number, $M_{1}$ is the rotor inlet Mach number, and $M_{2}$ is the rotor exit Mach number.

$$
\tau_{c}=1+\frac{(\gamma-1) M_{t} M_{t a n}}{\left(1+\frac{\gamma-1}{2} M_{2}^{2}\right)\left(1+\frac{\gamma-1}{2} M_{1}^{2}\right)} \sqrt{\tau_{c}}
$$

This equation is iteratively solved at each pitchwise location for $\tau_{c} . M_{t a n}$ and $M_{2}$ are measured by the 4 -way probe and contain noise from the 4 -way probe transducers. $M_{1}$ is measured using a wall static pressure transducer and the supply tank pressure, which both contribute to noise in its measurement shown in equation (3.5). The corrected mass flow is affected by the error in the measurement of $M_{1}$. Finally, $M_{t}$ is affected by the same transducer noise as $M_{1}$ and the error in the measurement of the shaft speed, $\omega$ shown in equation (3.6).

$$
\begin{gathered}
M_{1}=\sqrt{\frac{2}{\gamma-1}\left[{\frac{P_{\text {supply }}}{P_{\text {casing }}}}^{\frac{\gamma-1}{\gamma}}-1\right]} \\
M_{t}=\frac{\omega r_{\text {tip }}}{\sqrt{\frac{\gamma R T_{\text {supply }}}{1+\frac{\gamma-1}{2} M_{1}^{2}}}}
\end{gathered}
$$

Using the above errors in total pressure, static pressure, and flow angle, the 4-way probe gives an error of error $\left[\frac{T_{t 2}-T_{t 1}}{T_{t 1}}\right]= \pm 5.5 \%$ for calculation of the fractional total temperature ratio where $T_{t 2}$ is the rotor exit total temperature and $T_{t 1}$ is the rotor inlet total temperature. Using the errors for the total pressure and total temperature, the error in the adiabatic efficiency of the rotor is calculated to be error $\left[\eta_{\text {rotor }}\right]= \pm 4.8 \%$. Table 3.1 shows the results of the error analysis for measured quantities. 
Table 3.1: Results of the error analysis

\begin{tabular}{||l|c|}
\hline Parameter & Error \\
\hline Corrected Mass Flow & $1.5 \%$ \\
Corrected Speed & $1.0 \%$ \\
4-way probe tangential angle & $1 \mathrm{deg}$ \\
4-way probe radial angle & $2 \mathrm{deg}$ \\
4-way probe total Mach number & $2.5 \%$ \\
4-way probe total pressure & $1.0 \%$ \\
4-way probe static pressure & $2.0 \%$ \\
Fraction total temperature rise & $5.5 \%$ \\
Adiabatic efficiency & $4.8 \%$ \\
\hline
\end{tabular}

\subsection{Summary}

The aspirated fan stage was tested in the Blowdown Compressor facility which produced about 75 milliseconds worth of data during each test. This data was collected by data acquisition instrumentation that included high frequency response pressure transducers mounted in the casing walls and within the flowfield on the 4-way probe. The casing static pressure transducers are used to determine the inlet mass flow through the stage and estimate the overall performance of the stage. The 4-way probe provides the detailed flowfield information needed to determine the performance of the rotor and stator. A total and static pressure probe meassures the flow in the suction channel to determine how much suction flow is being removed from the core flow. An optical encoder provides the shaft speed data. All of this data is collected on a 24 channel data acquisition system that samples the data at 333 kiloHertz.

The error analysis performed on the data collection system shows that the total pressure can be measured by the 4 -way probe to within $1 \%$ of its value. Using the Euler equation, the fractional total temperature rise from the rotor can be calculated to within $3.9 \%$. Finally, using these errors in total temperature and total pressure, the adiabatic efficiency of the rotor can be measured to within $4.7 \%$ of its actual value. 


\section{Chapter 4}

\section{Aspirated Fan Stage Experiment}

\subsection{Introduction}

In this section results from high frequency instrumentation are presented. Two types of data are presented: (1) time-accurate measurements and (2) ensemble-averaged flow quantities. The ensemble-averaged flow quantities are compared with the APNASA analysis predictions, which, by assumption, are periodic with blade spacing. The time-accurate measurements show the blade to blade variations due to unsteadiness in blade coordinates and to rotorstator passing. Data was collected at five radial locations downstream of the rotor: $28 \%$, $47 \%, 65 \%, 84 \%$, and $92 \%$ span locations. Data was collected during the test time for 175 blade passes.

The experimental section of this thesis focuses on the data collected from the tests performed on a low speed aspirated stage designed to produce a pressure ratio of 1.59 at a blade speed of $750 \frac{\mathrm{ft}}{\mathrm{s}}$ with an adiabatic efficiency of $89 \%$. Detailed flowfield measurements describe the performance of the rotor and stator at design and off-design conditions. First, the design point data for both the rotor and stator are presented and compared with the APNASA analysis results. Pitchwise total pressure and flow angle measurements are shown at various spanwise locations. Total pressure contours are shown at the stator exit plane.

This chapter also presents experimental data on the off-design performance of the fan stage. Several different throttle plates are used to vary the mass flow along the $100 \%$ speed 
line ranging from $100 \%$ design flow $\left(64 \frac{\mathrm{lbs}}{\mathrm{s}}\right)$ to $79 \%$ design flow $\left(50 \frac{\mathrm{lbs} s}{\mathrm{~s}}\right)$ Stage total pressure and rotor wake measurements taken at the $65 \%$ span location $(0 \%$ span is the hub, $100 \%$ span is the tip) show the degradation of the stage performance as the stage mass flow is lowered from the design value. The stage shows a very large mass flow range without falling into a stalled condition compared to compressors producing similar pressure ratios.

A parametric view of the off-design performance suggests that the range of the compressor stage is partly due to the atypical blade shapes compared with conventional fan stages. The fact that the flow is turned past the axial direction in the lower section of the rotor reduces the loading on the lower half of the stator as the stage mass flow is decreased.

\subsection{Rotor Design Point Performance}

This section of the thesis presents the design point data for the rotor and stator exits. For the rotor, total pressure and flow angle measurements are presented as time-accurate data and as an ensemble-average of 7 rotor revolutions or 175 blade passes. Data is shown from the $28 \%, 47 \%, 65 \%, 84 \%$, and $92 \%$ span locations where $0 \%$ span represents the hub $100 \%$ span represents the casing. For comparison with APNASA, the data is corrected to $100 \%$ design speed and $100 \%$ design mass flow.

The data is taken at an axial distance of 1.1 inches downstream of the rotor tip section's trailing edge. The data collected at this location is compared with the APNASA analysis data corresponding to the same axial location. For all these plots, the pressure side is the left side of the wake, and the suction side is the right side of the wake. The blade to blade variation is shown including both the standard deviation of each blade passing and the standard deviation of each individual blade. 


\subsection{Design Point Total Pressure and Total Tempera- ture Spanwise Profiles}

Figure 4-1 presents the spanwise time-averaged variation of total pressure from the experiment compared with the APNASA analysis. The experiment shows good agreement with the APNASA analysis for the values of $\frac{r}{D_{t i p}}$ equal to $.387, .417, .445$. For the two measurements closest to the tip, $\frac{r}{D_{\text {tip }}}$ equal to .474 and .485 , the total pressure measured by the experiment is lower than the values predicted by APNASA. Unfortunately, the region of flow less than $\frac{r}{D_{t i p}}=.387$ is not included because the probe could not safely traverse that close to the hub.

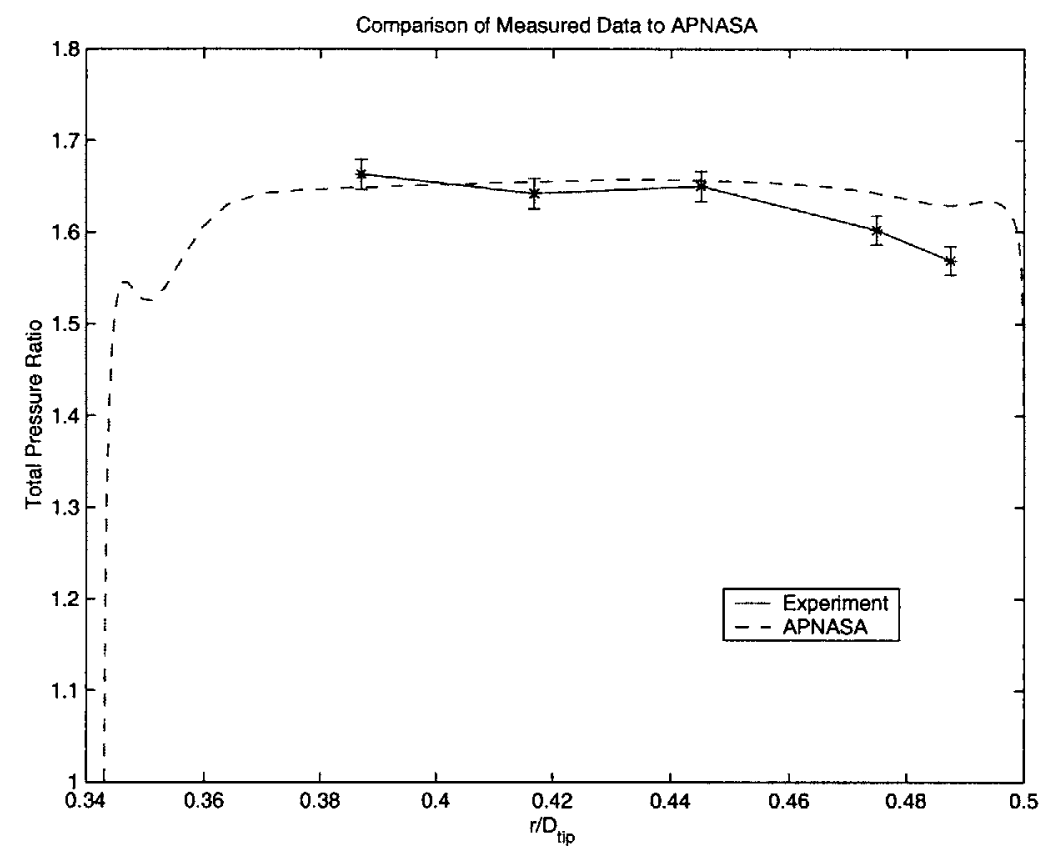

Figure 4-1: Comparison of experimental spanwise total pressure distribution with APNASA analysis

Figure 4-2 presents the spanwise time-averaged variation of total temperature from the experiment compared with the APNASA analysis. The total temperature is calculated from the Euler turbine equation in the form shown in equation (3.4). The experimental data shows that the rotor produces a lower total temperature than APNASA predicts in the lower half of the span while the agreement between the experiment and APNASA is good for the upper half of the span. Again, the measurement of total temperature is limited in the radial direction because the probe could not safely traverse that close to the hub. 


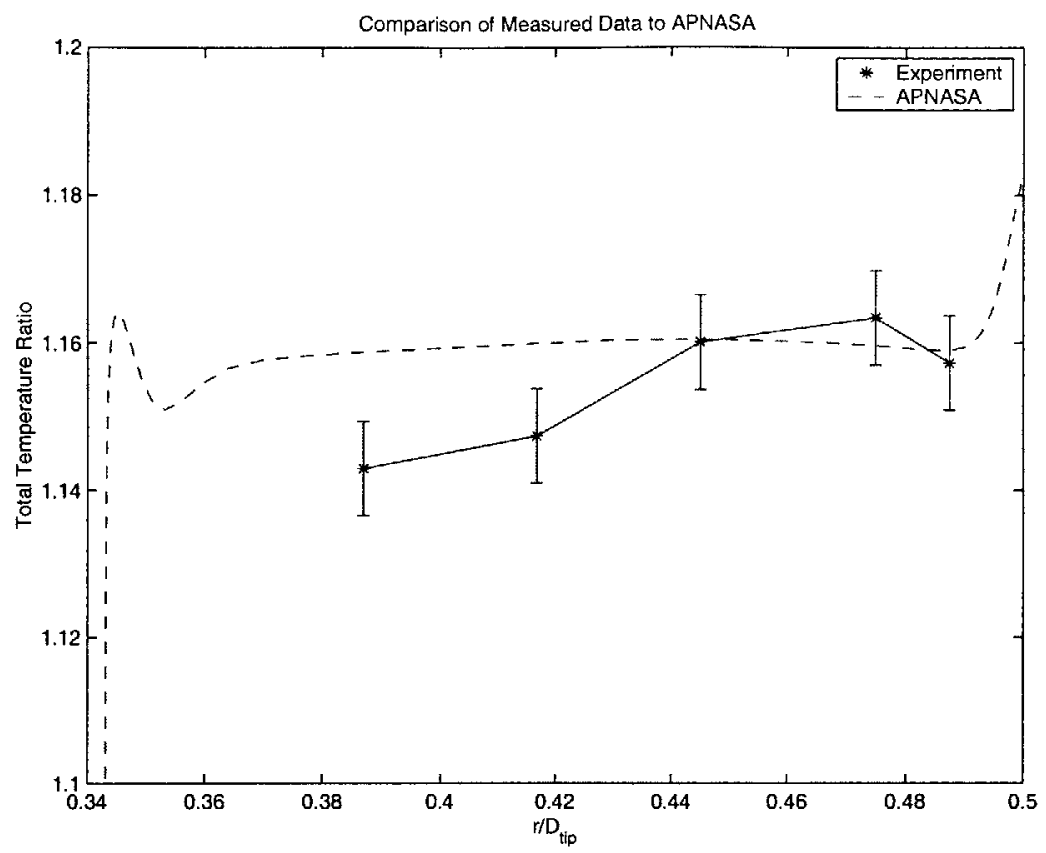

Figure 4-2: Comparison of experimental spanwise total temperature distribution with APNASA analysis

\subsubsection{8\% Span}

Figures 4-3 and 4-4 show the ensemble-averaged total pressure and tangential flow angle data of 175 blade passes, or 7 rotor revolutions, at the $28 \%$ span location. The ensemble-averaged data is compared with the APNASA analysis prediction at the same axial and spanwise position. The data is positioned such that the bottom of each wake corresponds to the same pitchwise position. In the total pressure plot, the predicted total pressure profile matches the experimental data very well within the core part of the flow. In describing the wake, the APNASA analysis predicts the wake width to be $20 \%$ of the pitch. The experimental data shows a slightly narrower wake of $15 \%$ percent of the blade pitch. The APNASA analysis also predicts a deeper total pressure deficit in the wake than the data shows. The analysis predicts a minimum total pressure ratio of 1.50 while the data shows the minimum total pressure ratio to be 1.57. Overall, the APNASA analysis predicts a lower total pressure ratio at this streamline than the data indicates.

Figure 4-4 shows that the APNASA analysis overpredicts the turning in the core part of the flow. The analysis predicts a relatively constant tangential flow angle of -49.5 degrees through the core flow. The experimental data shows a roughly linear decrease in turning 


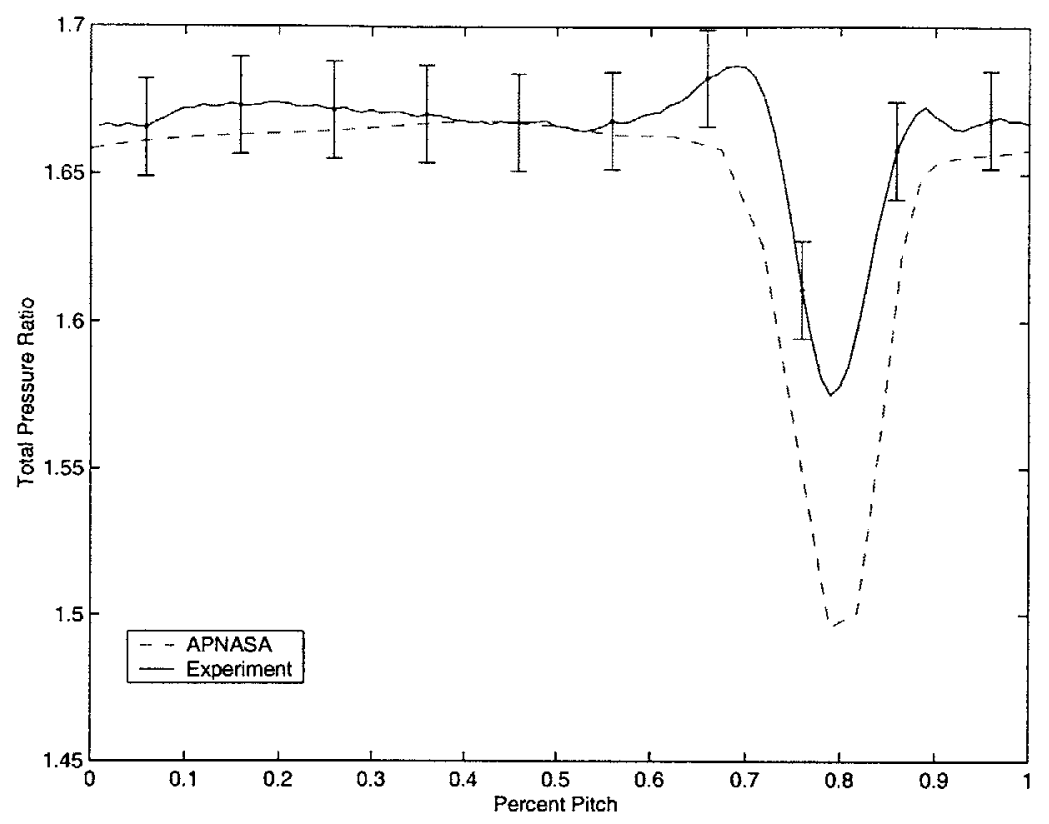

Figure 4-3: Pitchwise Total Pressure Profile for 28\% Rotor Span

from -49.5 degrees to a minimum turning of -45 degrees in the pressure side of the wake. In the wake flow, the APNASA analysis predicts an overturning of -54 degrees, and the experimental data only shows an overturning of -52.5 degrees.

Figure 4-5 shows the time-accurate data trace of total pressure and tangential flow angle for the $28 \%$ span location.

\subsubsection{7\% Span}

Figure 4-6 shows the ensemble-averaged total pressure data compared with the APNASA analysis prediction for the $47 \%$ span location. The APNASA analysis predicts the magnitude of the total pressure ratio in the core flow to be 1.66 which agrees with the experimental data. For the wake, the APNASA analysis and the experimental data agree on the wake width. The wake covers approximately $20 \%$ of the total blade pitch. In terms of the wake depth, the APNASA analysis predicts a minimum total pressure ratio of 1.48 while the experimental data only show a minimum total pressure ratio of 1.52 .

Figure 4-7 shows the ensemble-averaged tangential angle data compared with the APNASA analysis at the $47 \%$ span location. The APNASA analysis of the core flow shows a 


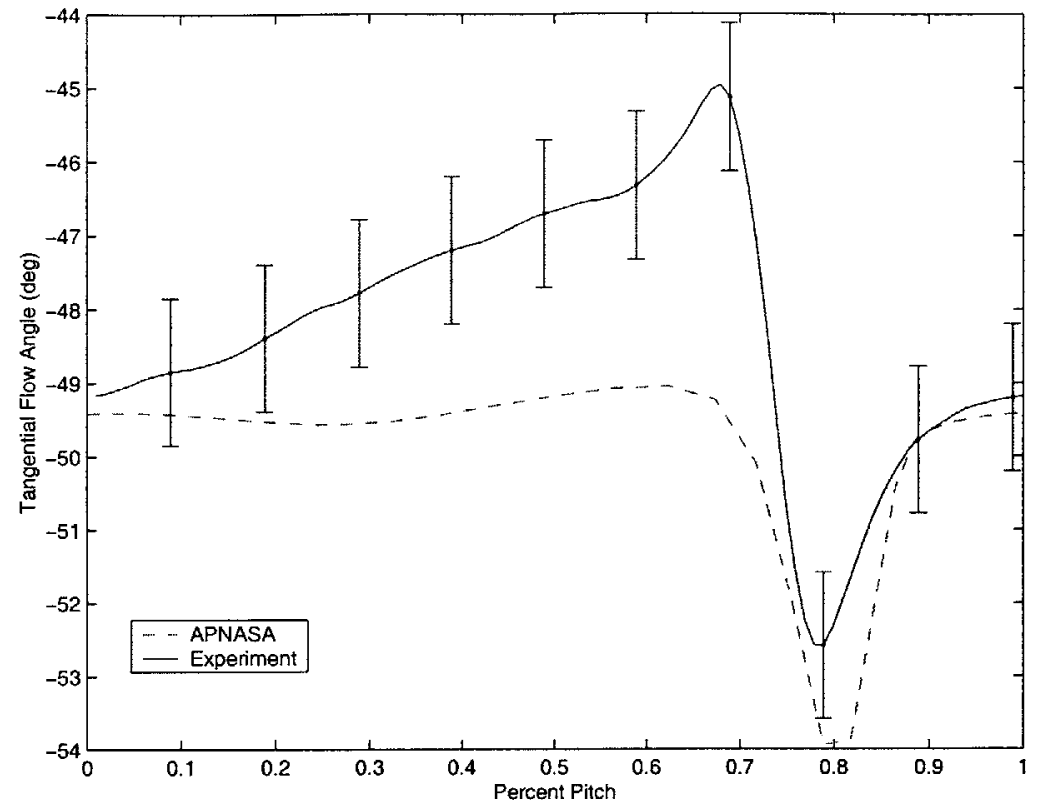

Figure 4-4: Pitchwise Tangential Angle Profile for 28\% Rotor Span
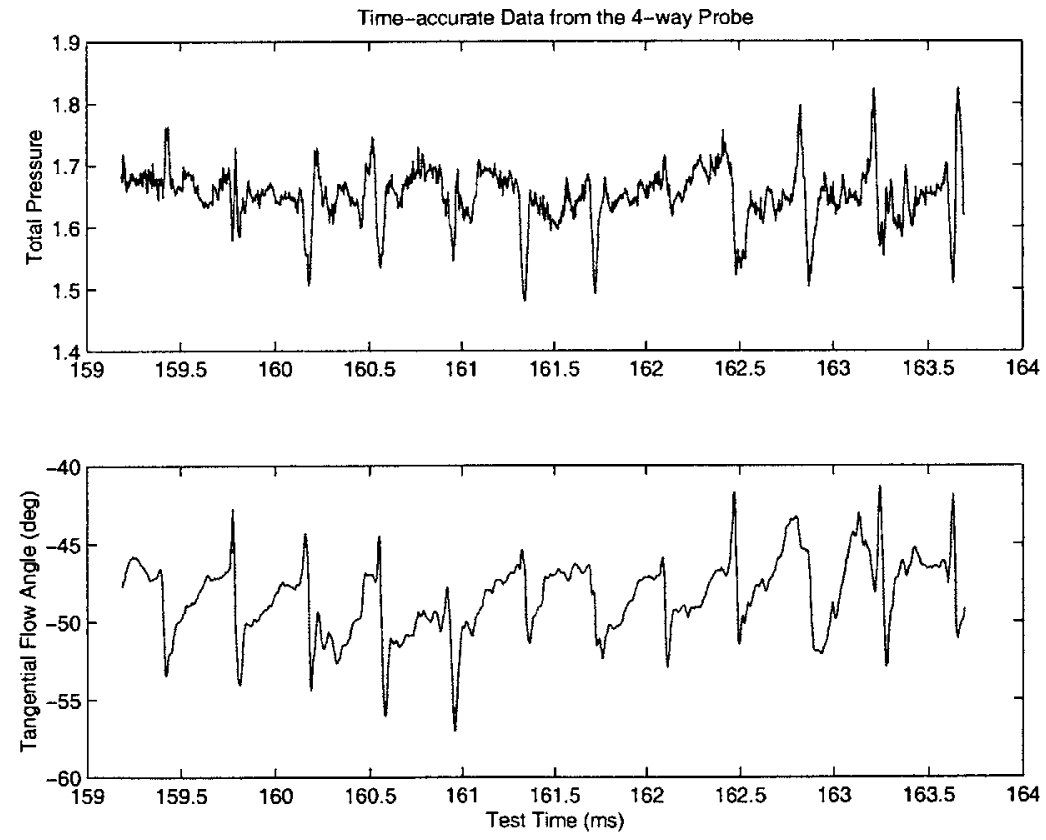

Figure 4-5: Time-Accurate Total Pressure and Tangential Angle for 28\% Rotor Span 


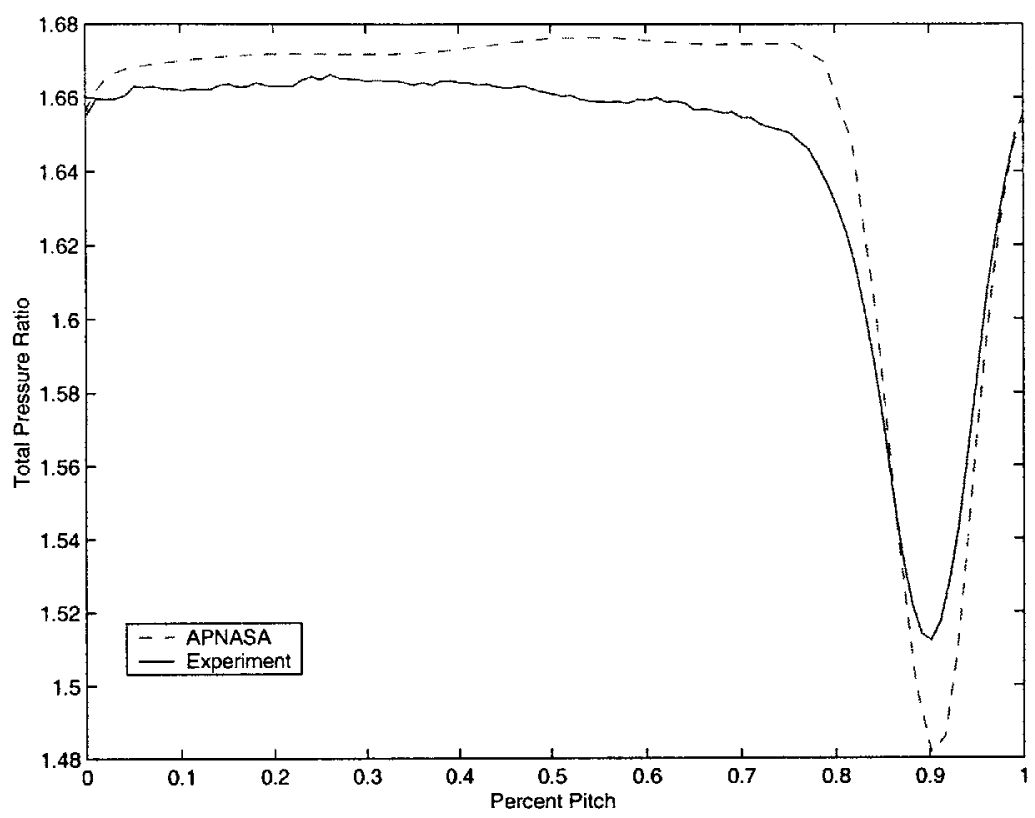

Figure 4-6: Pitchwise Total Pressure Profile for 47\% Rotor Span

nearly constant tangential angle of -47.5 degrees. The experimental data shows a core flow tangential angle that varies from -48 degrees to -46 degrees. Just on the pressure side of the wake, the experimental data shows an underturning of the flow peaking at -42.5 degrees compared to a predicted value of -47.5 degrees. As for the wake flow, the APNASA prediction and the experimental data agree very well on the amount of overturning in the wake. The experimental data shows a peak overturning of the flow of -52 degrees while the APNASA analysis predicts a slightly higher peak overturning of -53 degrees.

Figure 4-8 shows the time-accurate 4-way probe data from the test conducted with the probe at the $47 \%$ span location. Instantaneous total pressure and tangential flow angle are shown. A large amount of blade to blade variation exists in the time accurate data.

\subsubsection{5\% Span}

Figure 4-9 shows the ensemble-averaged total pressure data taken at the $65 \%$ span location compared with the APNASA analysis prediction for the same axial and radial location. The experimental data shows a total pressure ratio in the core flow of 1.66 to 1.68. The APNASA analysis predicts a total pressure ratio in the core flow of 1.67 to 1.68 . However, in the wake 


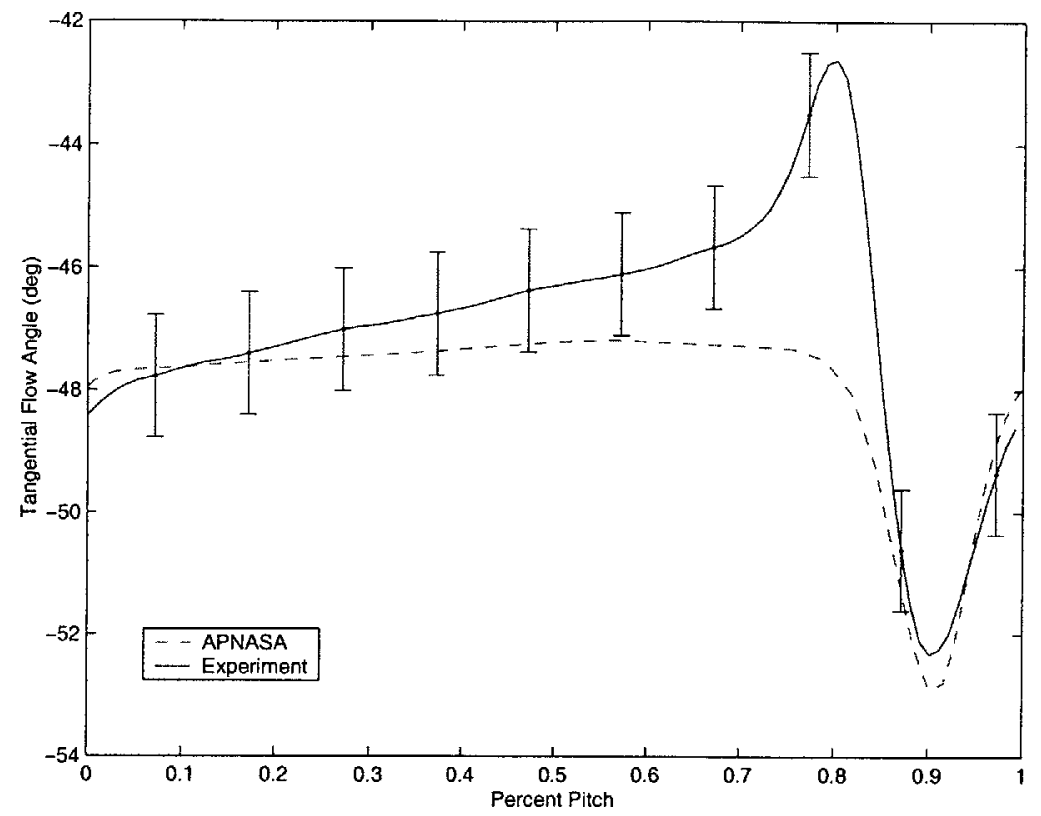

Figure 4-7: Pitchwise Tangential Angle Profile for 47\% Rotor Span
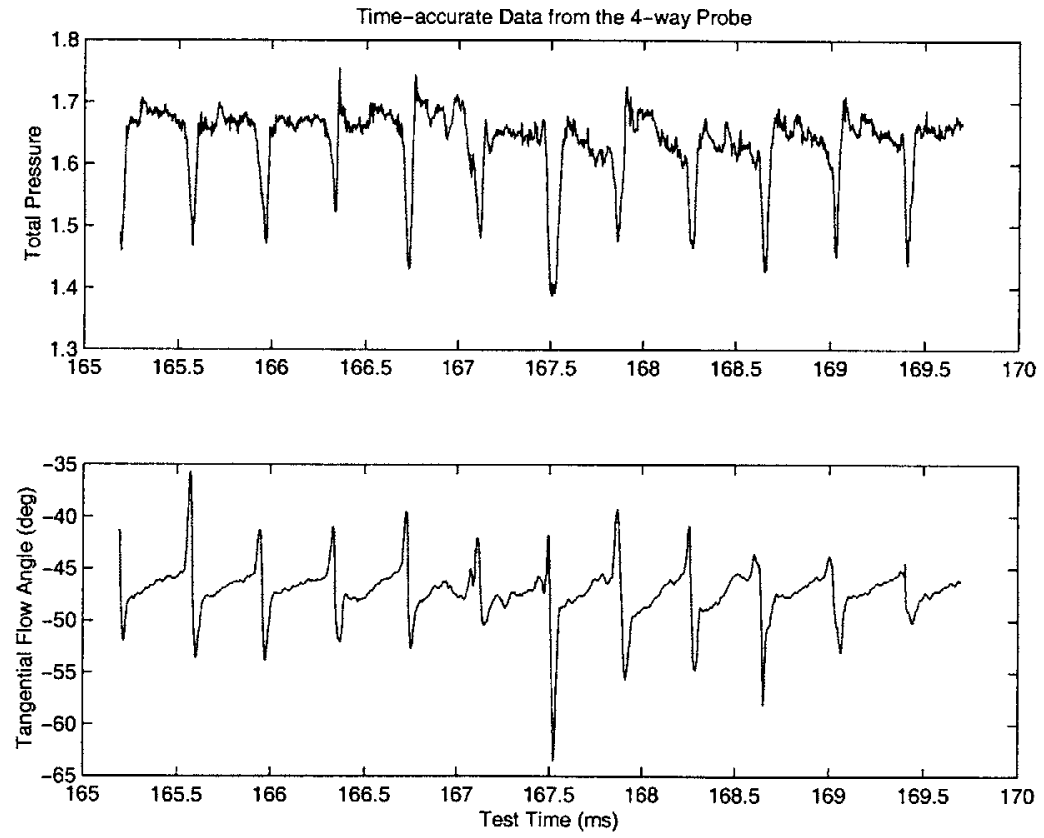

Figure 4-8: Time-Accurate Total Pressure and Tangential Angle for 47\% Rotor Span 
flow, the experimental data matches well with predictions. Both experiment and prediction show a wake width of approximately $20 \%$ of the blade pitch. As for the wake depth, the APNASA analysis predicts a minimum total pressure ratio in the wake flow of 1.47 , and the experimental data shows a minimum total pressure ratio of 1.52 in the wake flow.

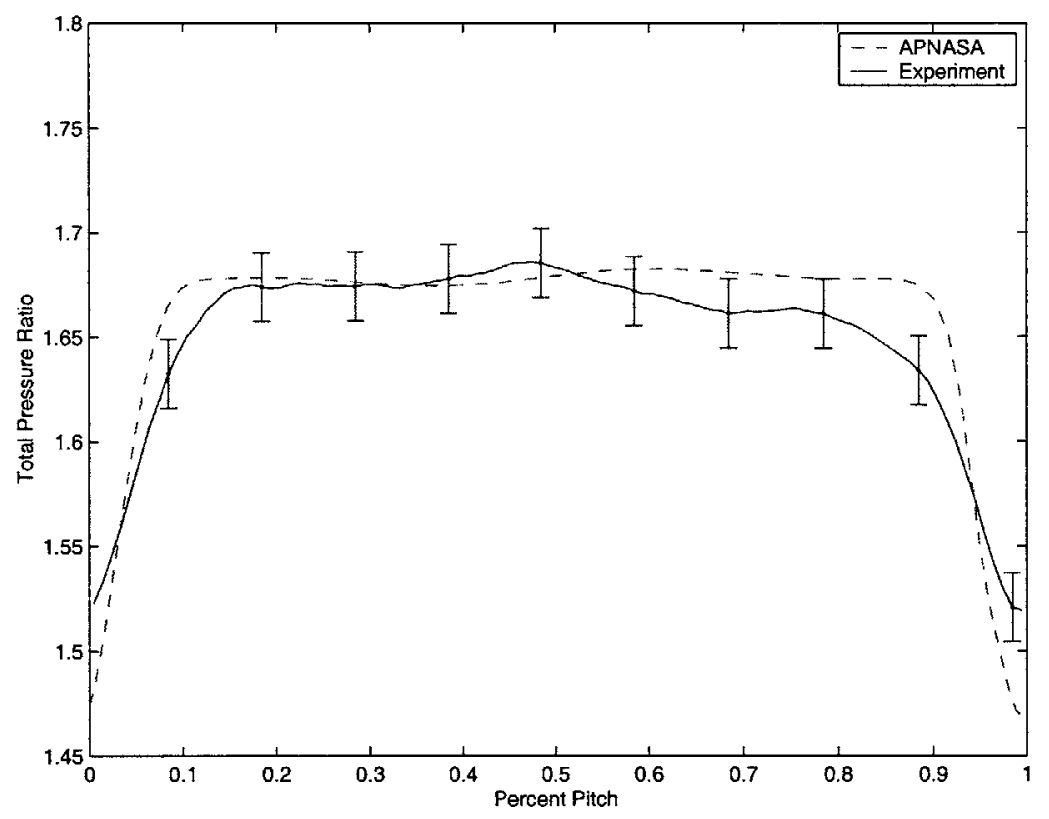

Figure 4-9: Pitchwise Total Pressure Profile for 65\% Rotor Span

Figure 4-10 shows the ensemble-averaged tangential angle data taken at the $65 \%$ span location. This data is compared with the APNASA analysis prediction for the same axial and radial location. For the core flow, the APNASA analysis and the experimental data differ by approximately two degrees. The experimental data ranges from approximately -46 degrees on the suction side of the wake to approximately -44 degrees of turning nearer the pressure side of the wake. The APNASA prediction shows a range from -42 degrees to -44 degrees of turning over this same amount of pitch. Once again, the experimental data shows an underturning to a peak of -41 degrees immediately to the pressure surface side of the wake. As for the wake flow, the APNASA analysis predicts a maximum overturning of -53 degrees compared to the experimental data which shows a peak overturning of -51 degrees.

Figure 4-11 shows the time-accurate total pressure and tangential flow angle data from the 4 -way probe at the $65 \%$ span location. 


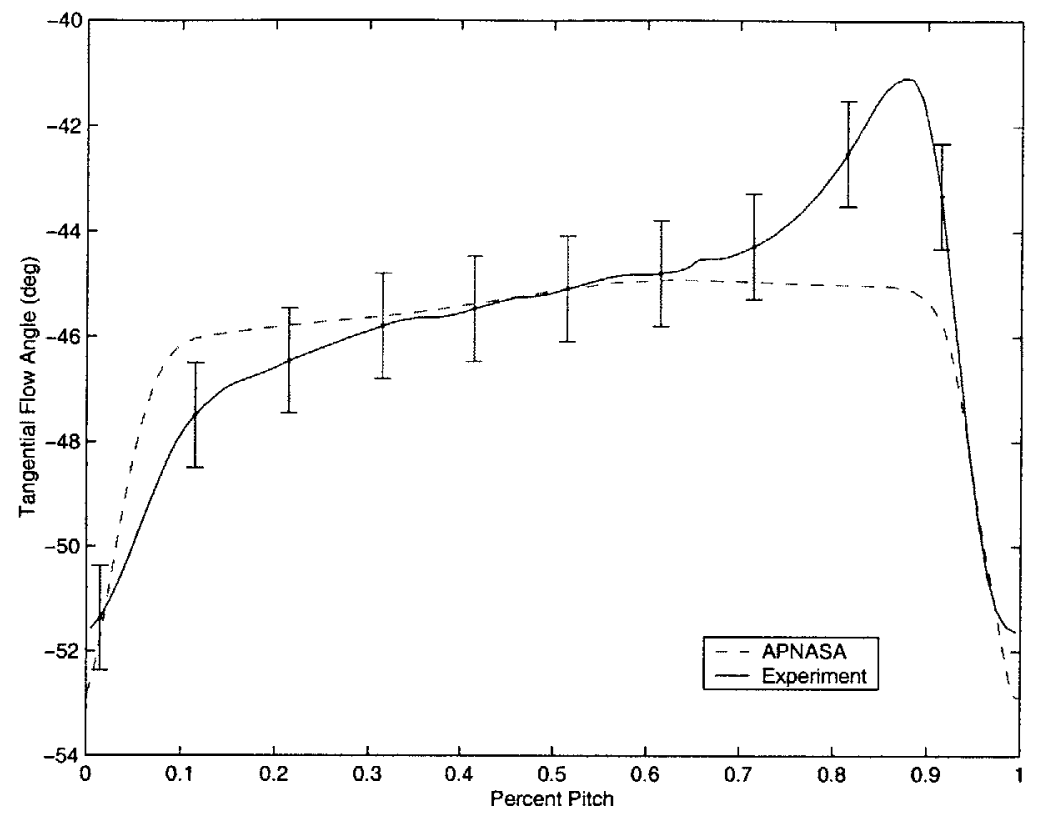

Figure 4-10: Pitchwise Tangential Angle Profile for 65\% Rotor Span
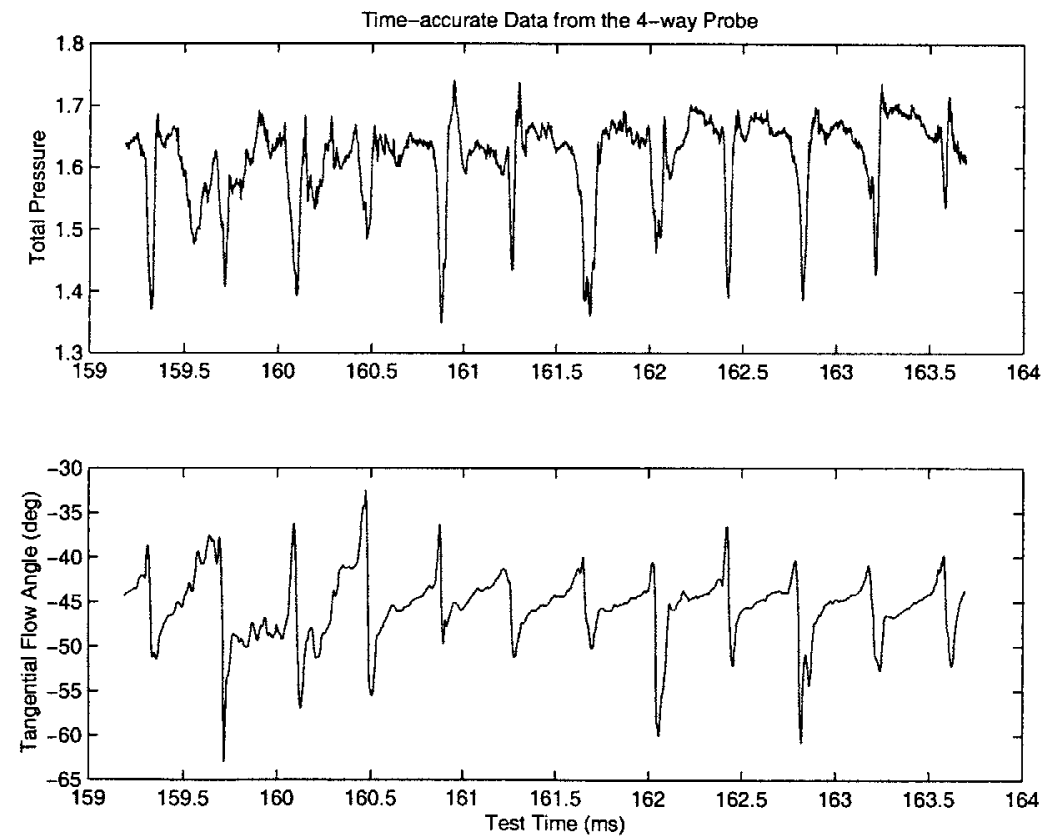

Figure 4-11: Time-Accurate Total Pressure and Tangential Angle for 65\% Rotor Span 


\subsubsection{4\% Span}

Figure 4-12 shows the ensemble-averaged total pressure ratio data taken at the $84 \%$ span location. This data is compared with the APNASA analysis data at the same radial and axial locations. The experimental data show a significant difference with the APNASA predictions both in the core flow and the wake flow. The experiment shows a core total pressure ratio of approximately 1.64 to 1.66 , and the APNASA analysis predicts a total pressure ratio of 1.66 for this radial location. For the wake flow, the experimental data shows a wake that extends for about $40 \%$ of the blade pitch, while the APNASA analysis predicts a wake width of only $20 \%$ of pitch. The depth of the total pressure deficit in the wake flow is also slightly different between the analysis and experiment. The APNASA analysis predicts a minimum total pressure ratio of 1.44 in the wake flow while the experiment shows a minimum total pressure ratio of 1.42 .

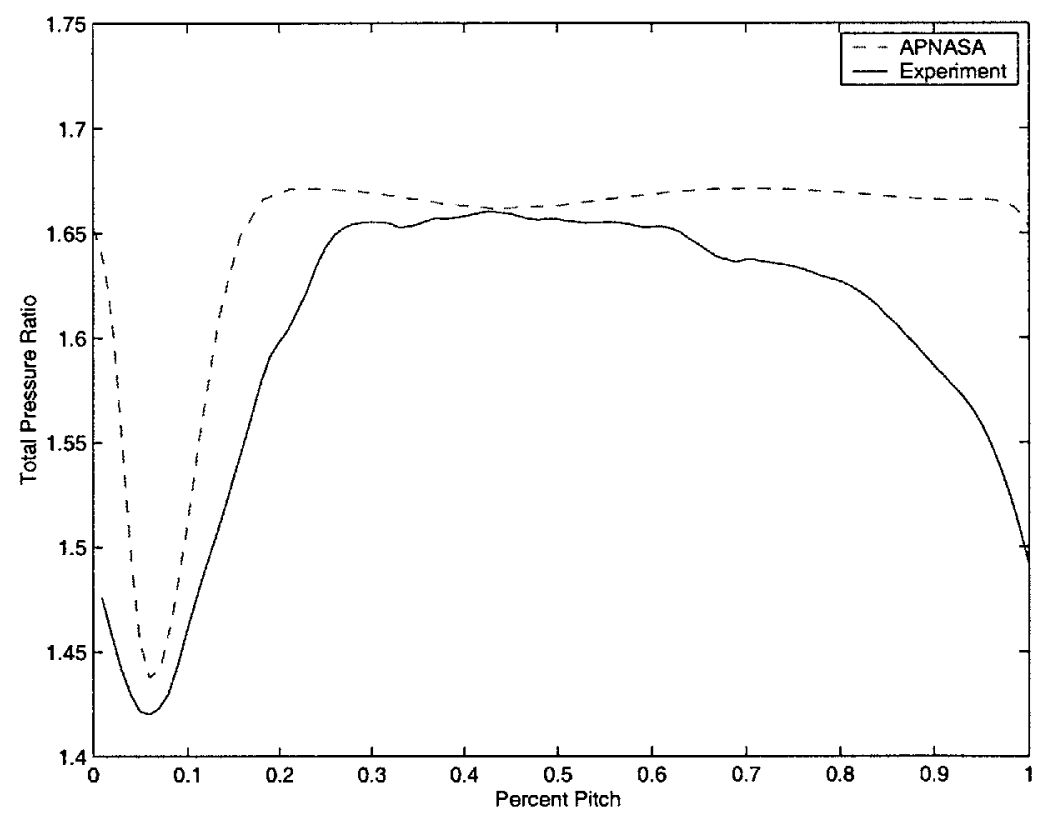

Figure 4-12: Pitchwise Total Pressure Profile for 84\% Rotor Span

Figure 4-13 shows the ensemble-averaged tangential angle data for the $84 \%$ span location. This data is compared to the APNASA analysis prediction for the same radial and axial locations. In the core flow, the experimental data ranges from -44 degrees to -40 degrees of turning. The predictions of the tangential flow angle from the APNASA analysis range from 
-44 degrees to -42 degrees in the core flow. Once again, the experimental data shows an underturning to -39 degrees just to the pressure side of the wake. Regarding the wake flow, the APNASA analysis predicts a maximum overturning of -53 degrees. In the experimental data, the wake flow shows a maximum overturning of -56 degrees.

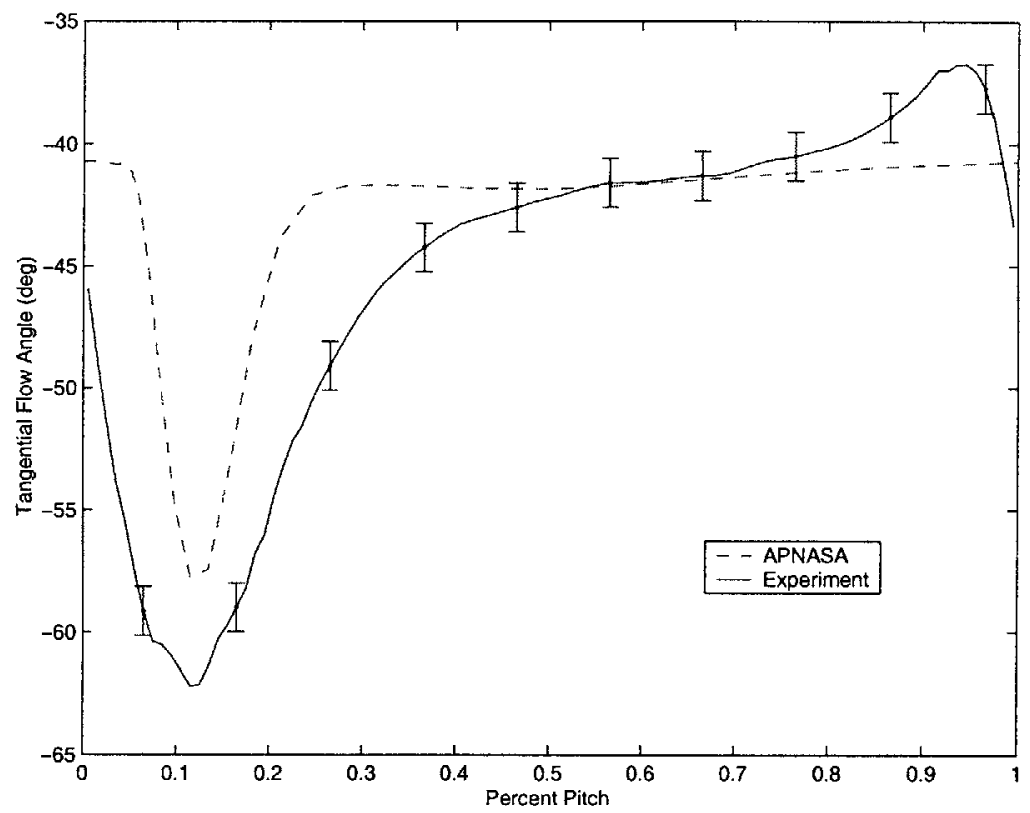

Figure 4-13: Pitchwise Tangential Angle Profile for 84\% Rotor Span

Figure 4-14 presents the time-accurate total pressure and tangential angle data at the $84 \%$ span location.

\subsubsection{2\% Span}

Figure $4-15$ shows the ensemble-averaged total pressure ratio data from the $92 \%$ span location. This data is compared with the APNASA analysis predictions from the same radial and axial locations. This figure shows the dramatic difference between the wake profiles of the APNASA analysis and the experimental data. For the core flow, the experimental data shows the total pressure ratio varying from 1.67 to 1.62. The APNASA calculation again predicts a constant total pressure ratio of 1.66. The real difference between the two lies in the wake width. The APNASA analysis again predicts a wake width of $20 \%$ pitch while the experimental data shows a wake width of approximately $40 \%$ of the blade pitch. The 

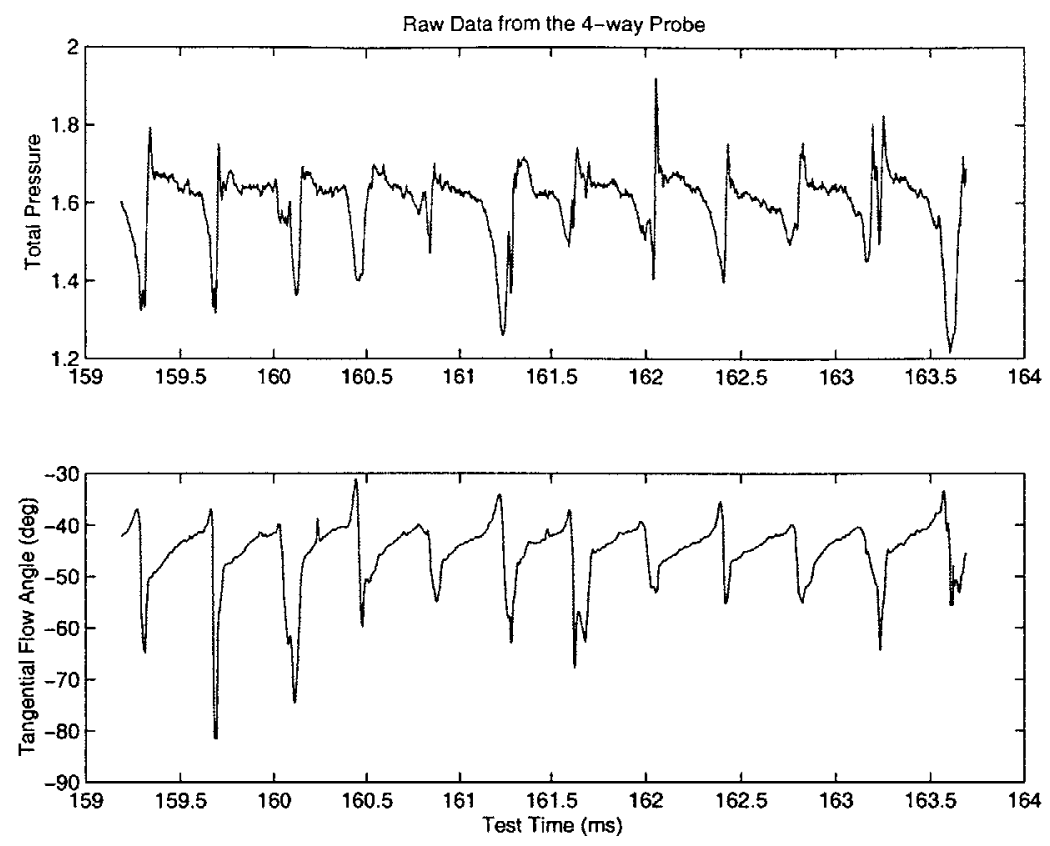

Figure 4-14: Time-Accurate Total Pressure and Tangential Angle for 84\% Rotor Span

analysis and the data do agree well with determining the depth of the wake. The APNASA analysis predicts a minimum total pressure ratio of 1.37 , and the experimental data shows a minimum total pressure ratio of 1.35 .

Figure 4-16 shows the ensemble-averaged tangential angle data for the $92 \%$ span location and compares the data to the predictions of the APNASA analysis at the same axial and radial locations. For the core flow, the experimental data shows a tangential flow angle ranging from -44 degrees to -40 degrees. The APNASA analysis predicts a tangential flow angle only ranging from -41 degrees to -42 degrees for the core flow. Again the experimental data shows a mild underturning of the flow to -37 degrees near the pressure side of the wake. Within the wake flow, the APNASA analysis predicts a maximum overturning of the flow of -57 degrees. For the experiment data, the maximum overturning of the flow is -62 degrees.

Finally, figure 4-17 presents the instantaneous total pressure and tangential angle measurements from the 4 -way probe at the $92 \%$ span location. 


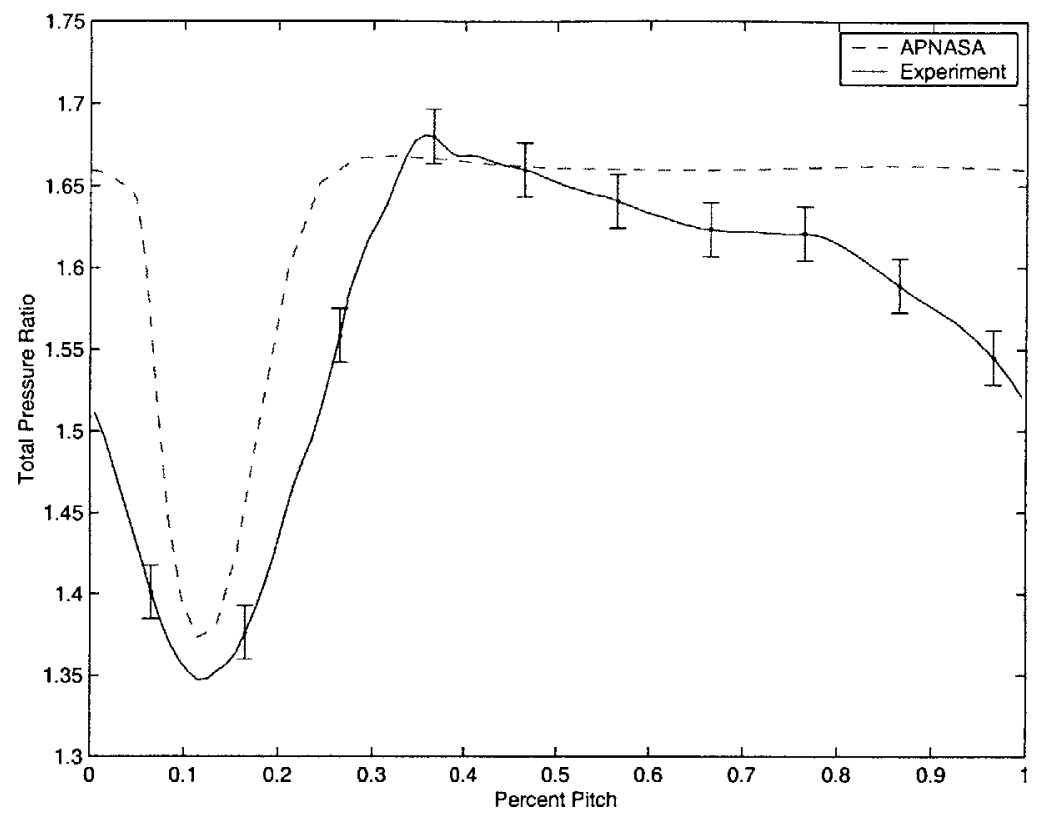

Figure 4-15: Pitchwise Total Pressure Profile for 92\% Rotor Span

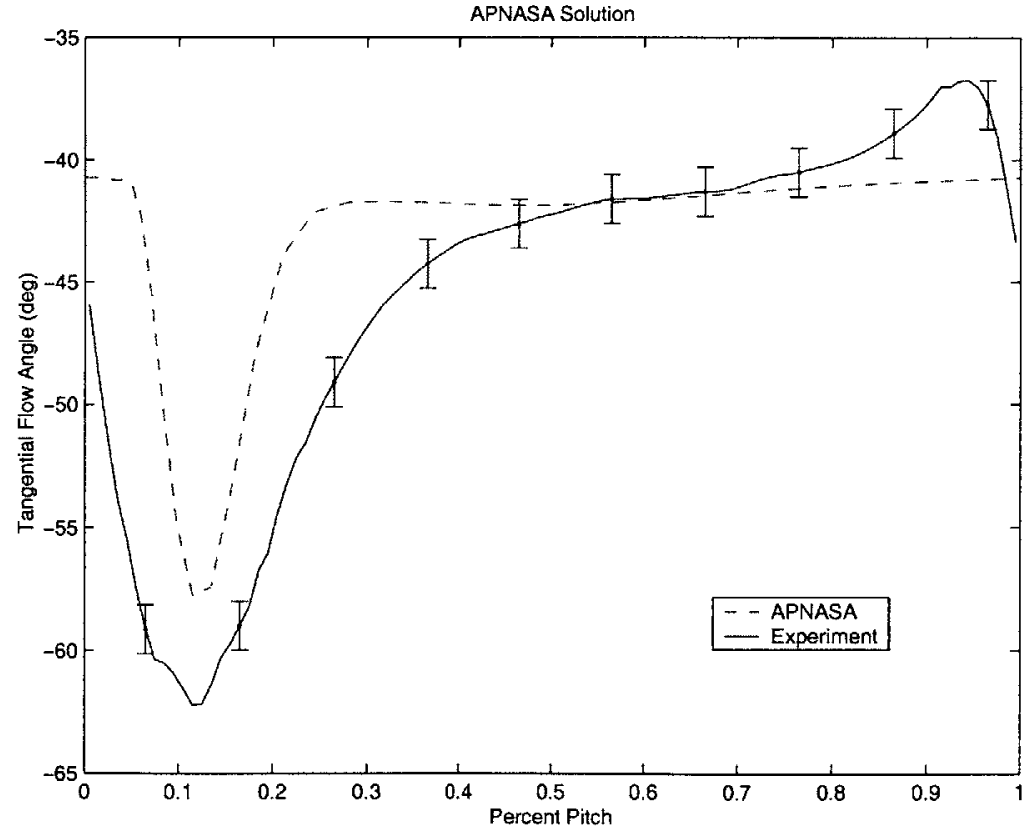

Figure 4-16: Pitchwise Tangential Angle Profile for 92\% Rotor Span 

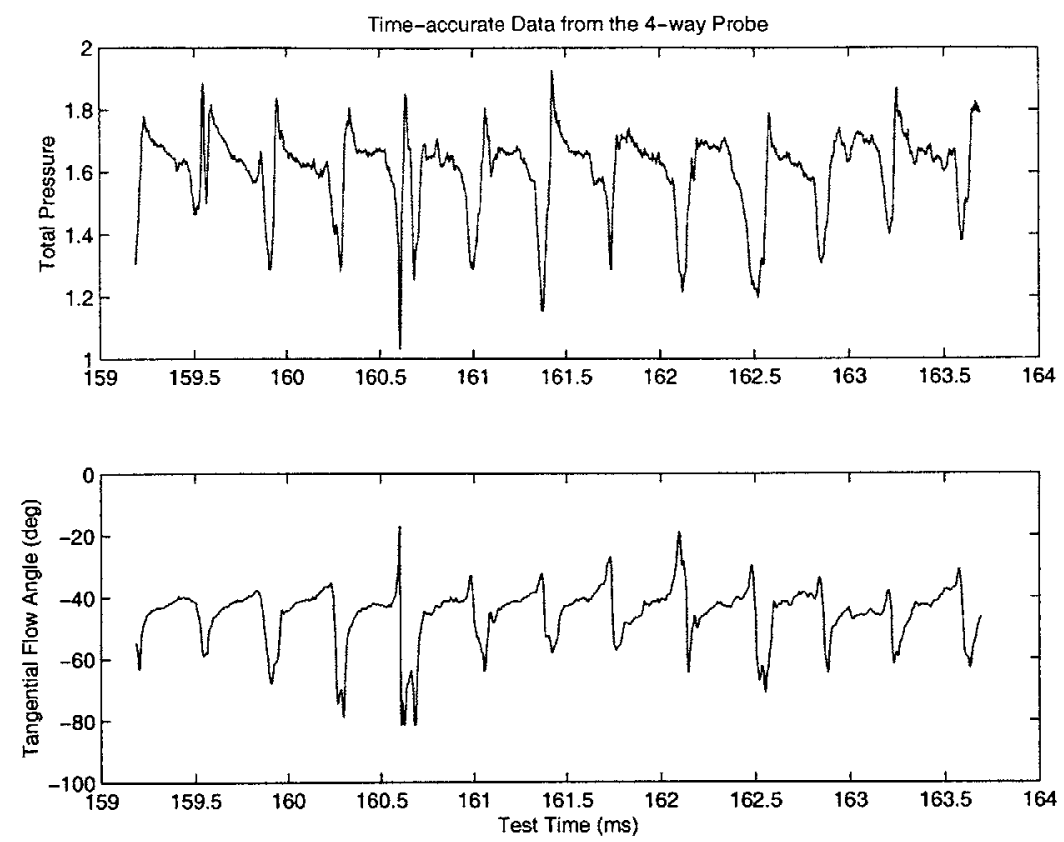

Figure 4-17: Time-Accurate Total Pressure and Tangential Angle for 92\% Rotor Span

\subsubsection{Blade to Blade Variation}

As can be seen in the time-accurate data traces in the previous section, the individual rotor blades show a significant unsteadiness from one blade passing to the next. In reference [4], Gertz presents data from three different transonic compressor stages: the NASA 1st rotor of a two-stage compressor, AFAPL (Air Force Aero Propulsion Lab) rotor by Wennerstrom, and a Pratt and Whitney Split-Flow fan. He states that all three rotors showed significant amounts of unsteadiness in the wake flow including as much as $5 \%$ to $15 \%$ excesses or deficits in the mean total pressure levels. This section presents the blade to blade variation of total pressure and tangential flow angle for different span locations.

Figure 4-18 shows the blade to blade variations of total pressure for the $28 \%$ span location. In the core flow, the total pressure profile exhibits a mild blade to blade variation of $3 \%$ of the mean total pressure at that pitch location. The wake flow shows a much larger blade to blade variation. Specifically, the two sigma blade to blade variation is $10 \%$ of the mean value for a pitch location.

Figure 4-19 shows the blade to blade variation in total pressure for the $65 \%$ span location. In the core flow, the total pressure variation is $4 \%$ of the mean value. Again, the wake flow 


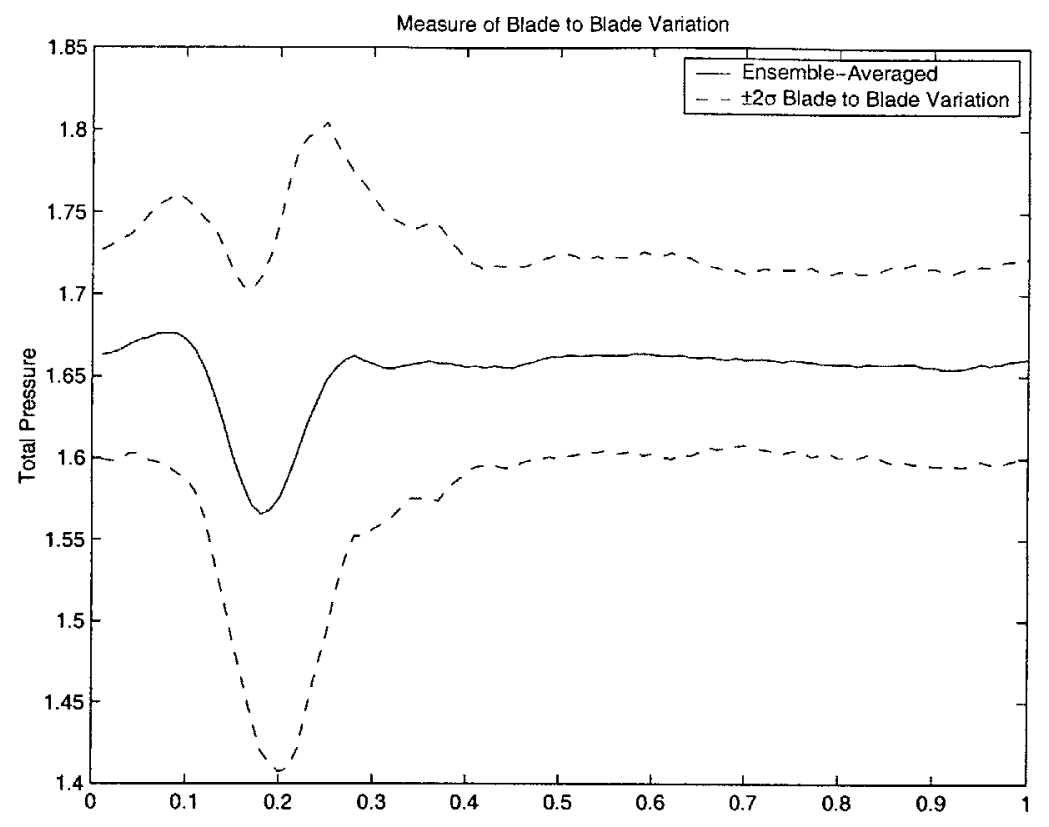

Figure 4-18: Blade to Blade Variation in the Total Pressure data for 28\% Rotor Span shows much more blade to blade variation. The experimental data shows a variation of up to $15 \%$ of the mean value at the pitch location.

Figure 4-20 shows the blade to blade variation in total pressure for the $92 \%$ span location. In the core flow, the blade to blade total pressure variation is $3 \%$ of the mean value. The wake flow shows a much larger variation. The data shows the wake to have a $30 \%$ variation from the mean value at the pitch location.

The significance of these fluctuations will be discussed and elaborated on in chapter 5 . 


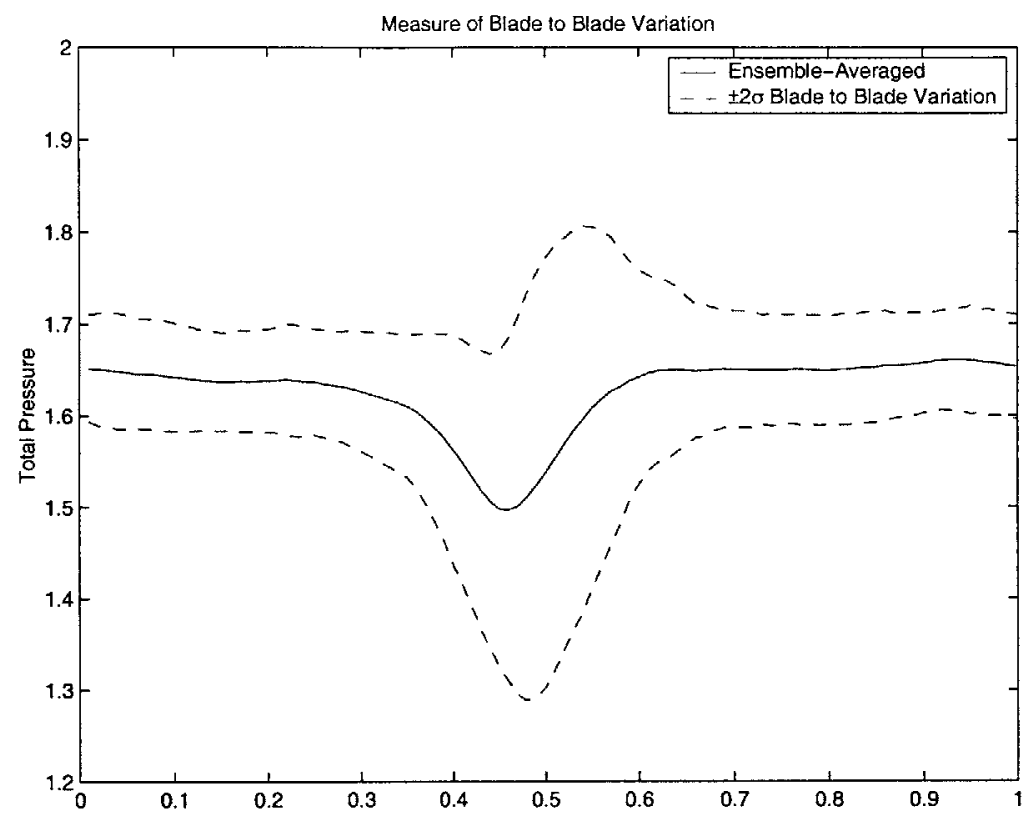

Figure 4-19: Blade to Blade Variation in the Total Pressure data for 60\% Rotor Span

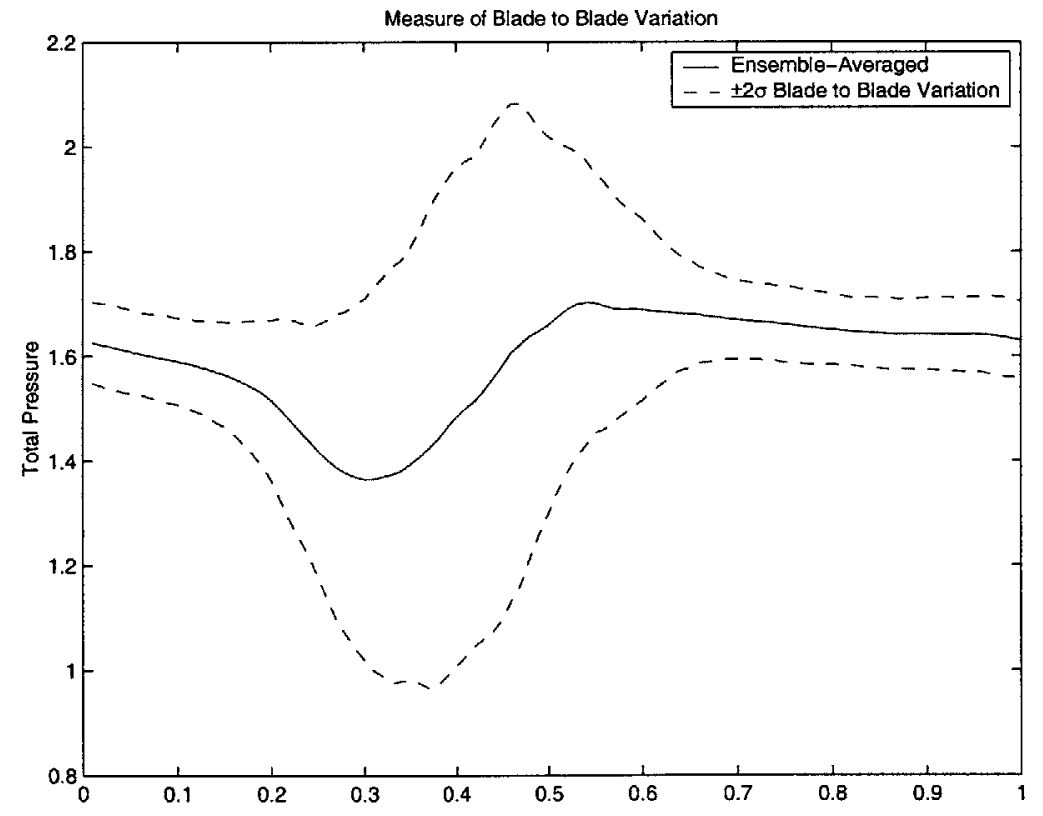

Figure 4-20: Blade to Blade Variation in the Total Pressure data for $92 \%$ Rotor Span 


\subsection{Stator Performance}

The stator performance is most effectively characterized by the total pressure at the exit plane. Experimental data was collected in a grid of four spanwise points by 6 pitchwise points located approximately one inch downstream of the stator trailing edge. The four spanwise points are located at $20 \%, 40 \%, 60 \%$, and $80 \%$ span where $0 \%$ span is the hub and $100 \%$ span is the casing. The six pitchwise points are clustered around the wake with two points in the main flow. The points are located at $0 \%, 5 \% 30 \%, 80 \%, 90 \%$, and $95 \%$ pitch where $0 \%$ pitch is the pressure side of the wake and $99 \%$ pitch is the suction side of the passage.

Figure 4-21 shows the total pressure contour from the APNASA analysis at the same axial location as the 4-way probe location. The suction side of the stator passage is located on the left hand side of the contour plot while the pressure surface side is on the right hand side of the plot. The contour plot shows two low total pressure regions near the endwalls on the suction side of the wake. These low total pressure regions are the largest contributors of loss in the stator.

Figure 4-22 shows the time-averaged value of total pressure measured at each location with a contour plot superimposed over the measured values. The same low total pressure buckets near the suction surface of the wake are seen in the data. The size, position, and depth of the 'lossy' regions are close to the APNASA analysis. 


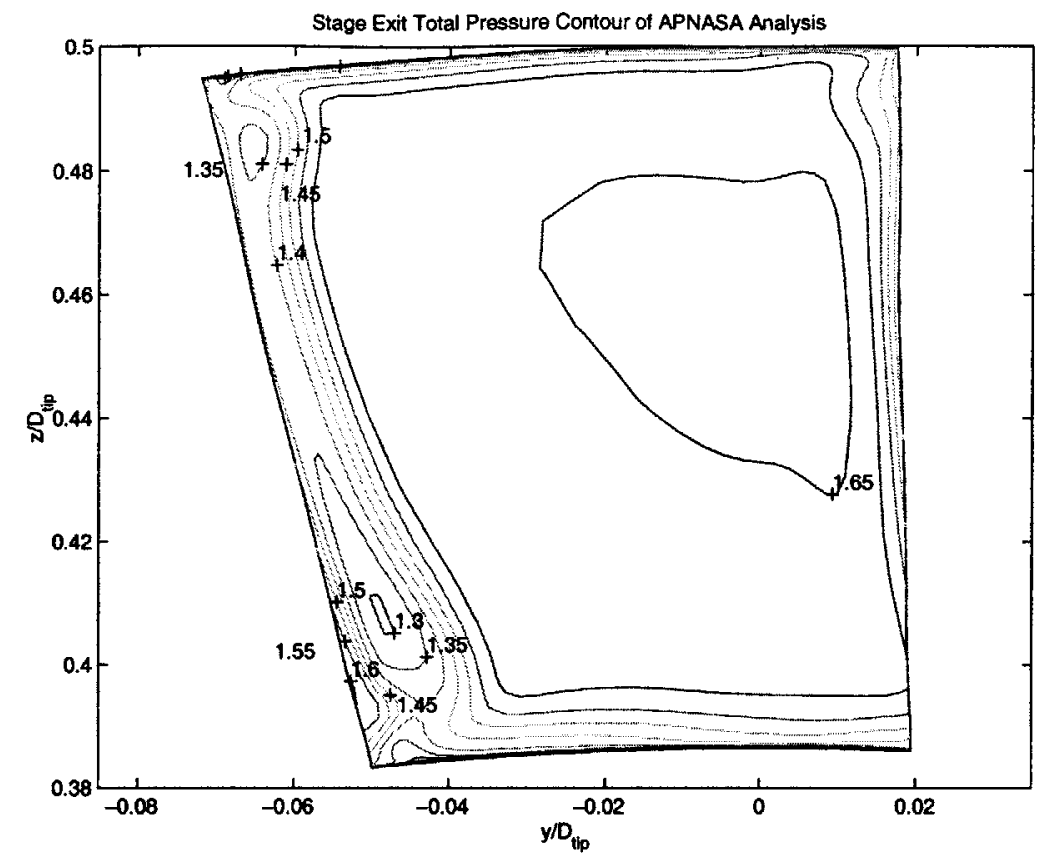

Figure 4-21: Stator exit total pressure contour predicted by APNASA

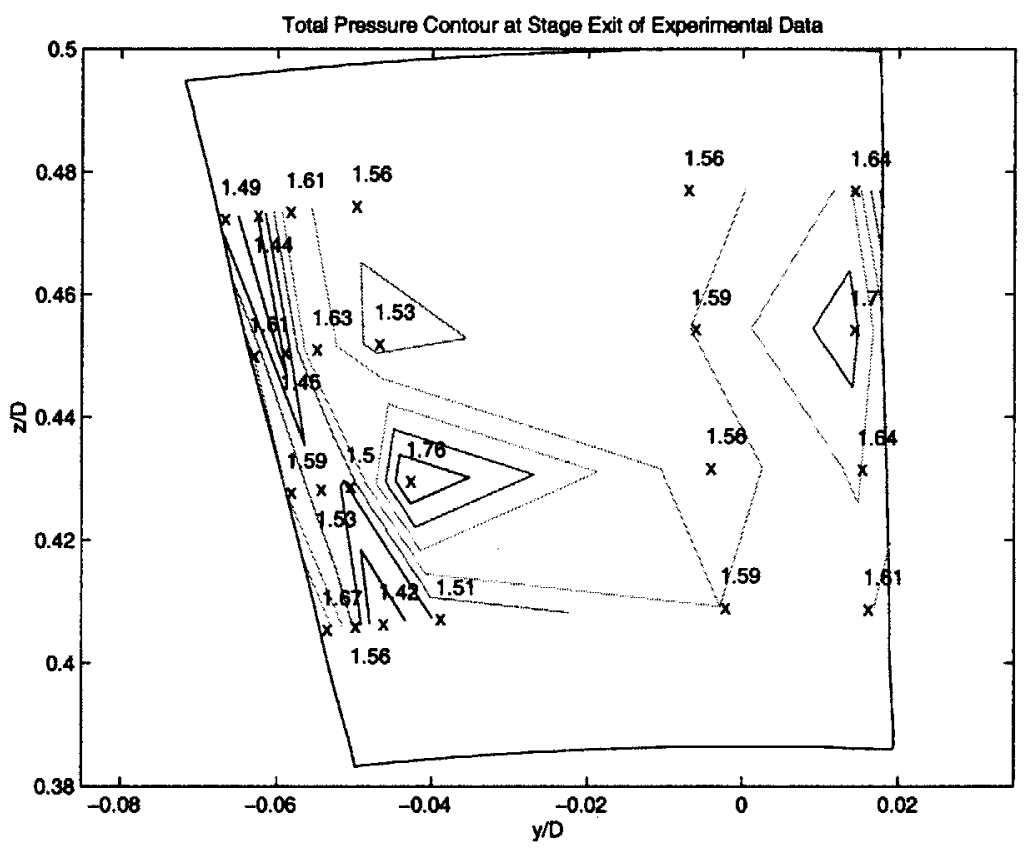

Figure 4-22: Time-averaged total pressure data from stator exit 


\subsection{Measured Stage Suction}

This section presents the stage suction measured in the channels shown in figure 2-16. Three suction channels in the casing insert carry the suction flows from the rotor and stator to the dump tank. A probe measures the total and static pressure a point in the channel. The total temperature is estimated using equation (3.3). Knowing the cross-sectional area of the channel and the number of channels, the total mass flow through the channels can be calculated. The stage suction was not changed between runs.

Figures 4-23, 4-24, and 4-25 show the time-accurate measurements of mass flow measured in the suction channels. The data shows that the rotor casing suction is lower than the design value presented in tables (2.4) and (2.5). The measured rotor blade suction matches well with the design value. The measured stator suction is approximately $45 \%$ of the design value. Table (4.1) shows the comparison of measured and design suction values in the channel.

Table 4.1: Rotor Suction Requirements

\begin{tabular}{||l|c|c|}
\hline & Design & Measured \\
\hline \hline Rotor Casing Suction & $1.29 \%$ & $0.72 \%$ \\
\hline Rotor Blade Suction & $0.95 \%$ & $0.84 \%$ \\
\hline Stator Casing and Blade Suction & $1.95 \%$ & $0.85 \%$ \\
\hline
\end{tabular}




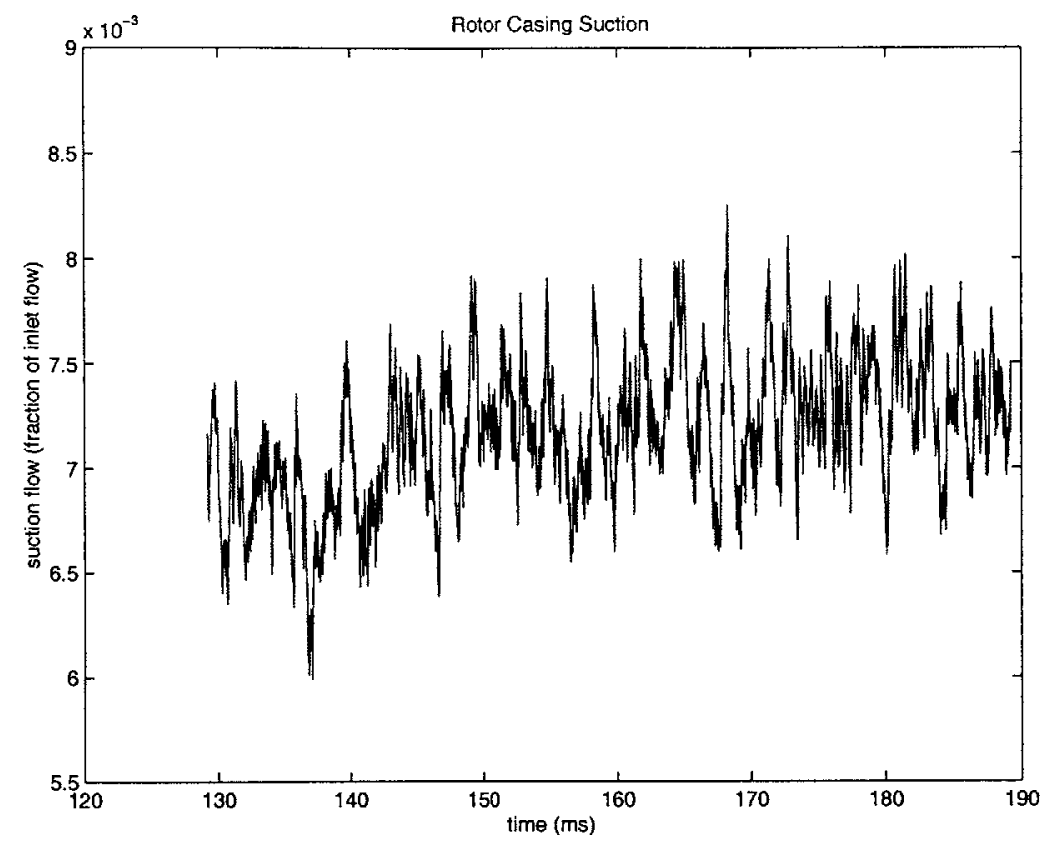

Figure 4-23: Time accurate measurement of rotor casing suction

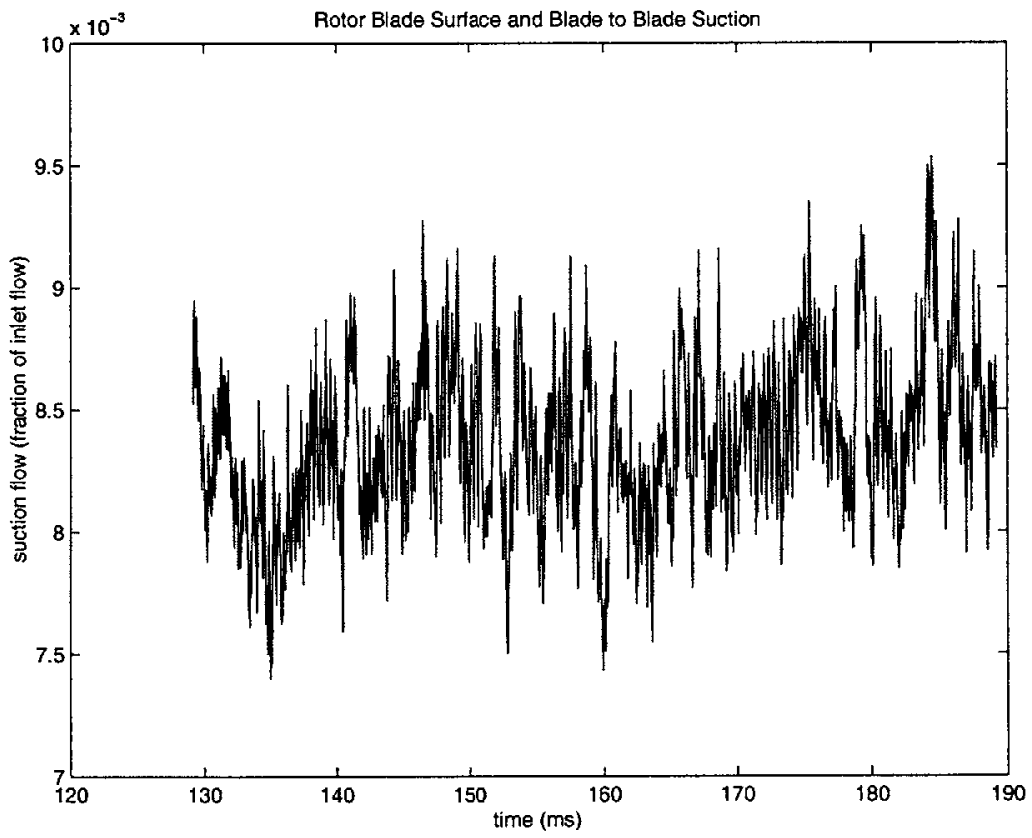

Figure 4-24: Time accurate measurement of rotor blade suction 


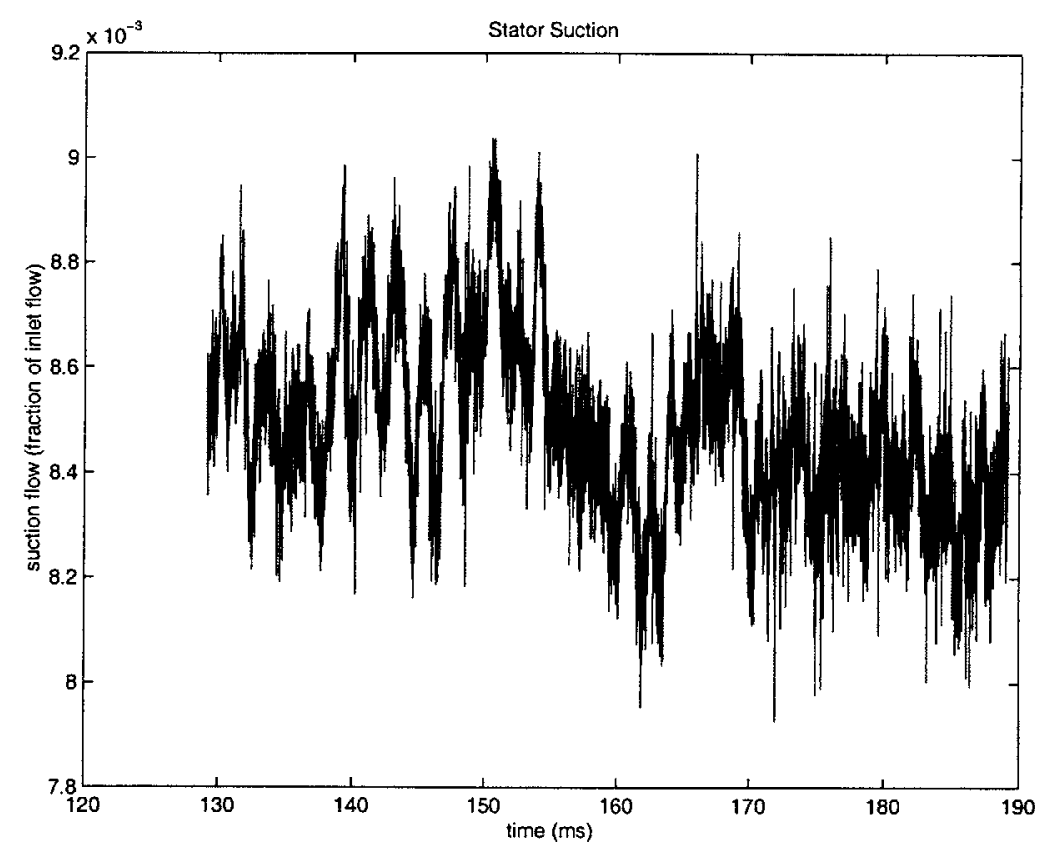

Figure 4-25: Time accurate measurement of stator suction 


\subsection{Off-design Performance}

Experimental tests of the off-design performance of the aspirated fan stage show that the stage produces significant pressure ratio down to $79 \%$ design mass flow without entering into a rotating stall regime. Figure 4-26 shows the experimental off-design performance compared with APNASA and Pratt \& Whitney [18] fully viscous analyses. The predictions given by the analysis codes show slightly higher stage pressure ratios than those measured in the off-design experiments.

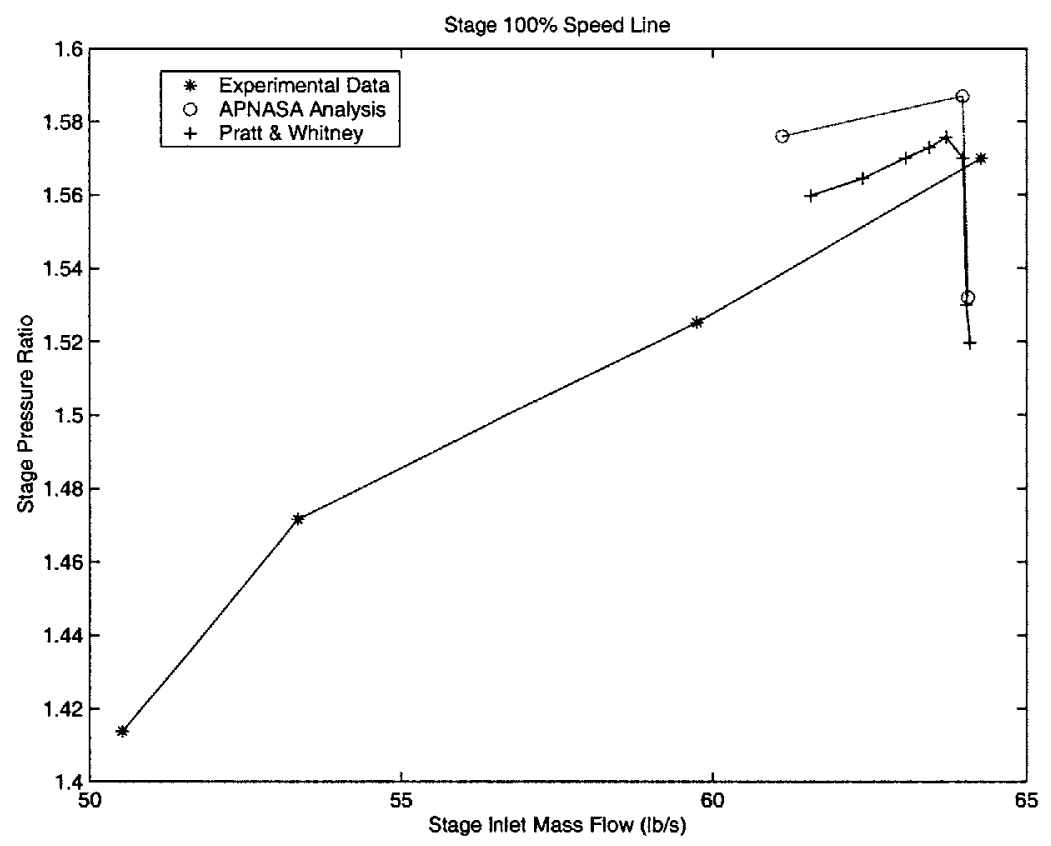

Figure 4-26: Off-design performance of Aspirated Fan Stage

Figure 4-27 presents the ensemble-averaged total pressure variation across the pitch at the $64 \%$ spanwise location downstream of the rotor. The total pressure wakes of the rotor get wider and deeper as the mass flow decreases. For the design mass flow, the rotor wake covers approximately $15 \%$ of the blade pitch, where as the $79 \%$ mass flow test shows the wake covering approximately $50 \%$ of the blade pitch.

Figure 4-28 shows the time accurate total pressure data taken at $40 \%$ span and $5 \%$ pitch downstream of the stator for design mass flow and $79 \%$ design mass flow runs. The lower mass flow run shows higher variation than the design mass flow run but still no evidence of rotating stall. 


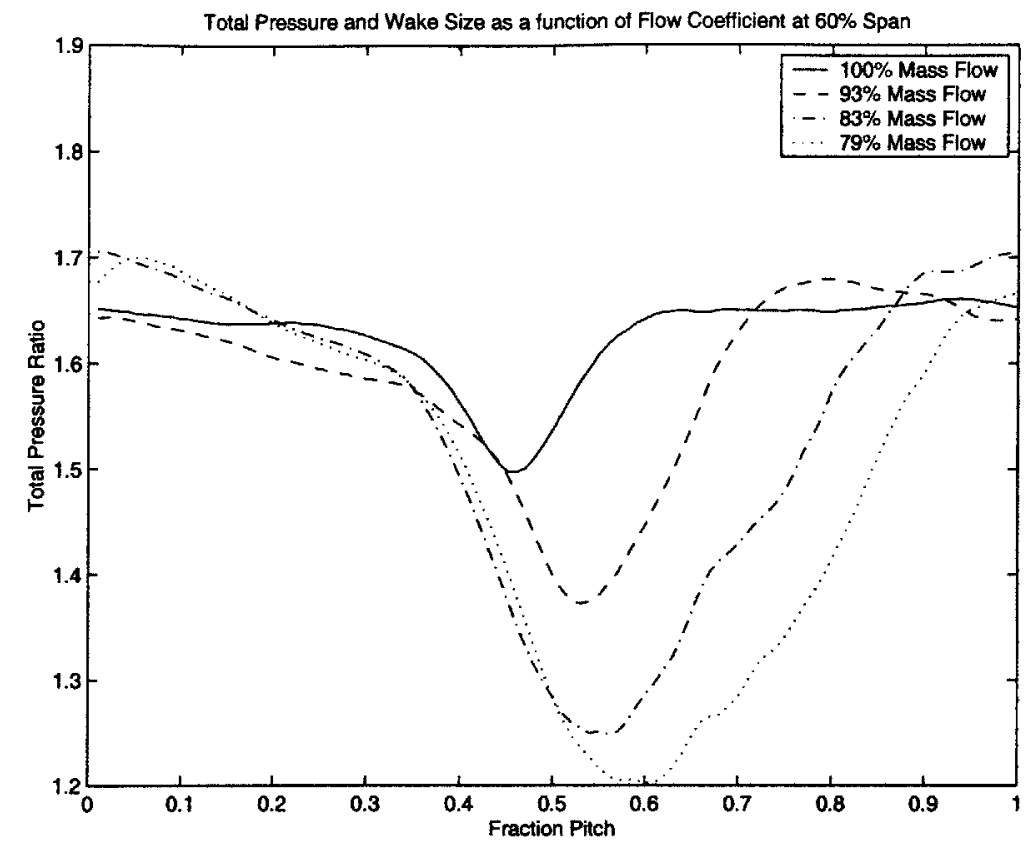

Figure 4-27: Pitchwise Total Pressure at Rotor Exit for 65\% Span at different Mass Flows
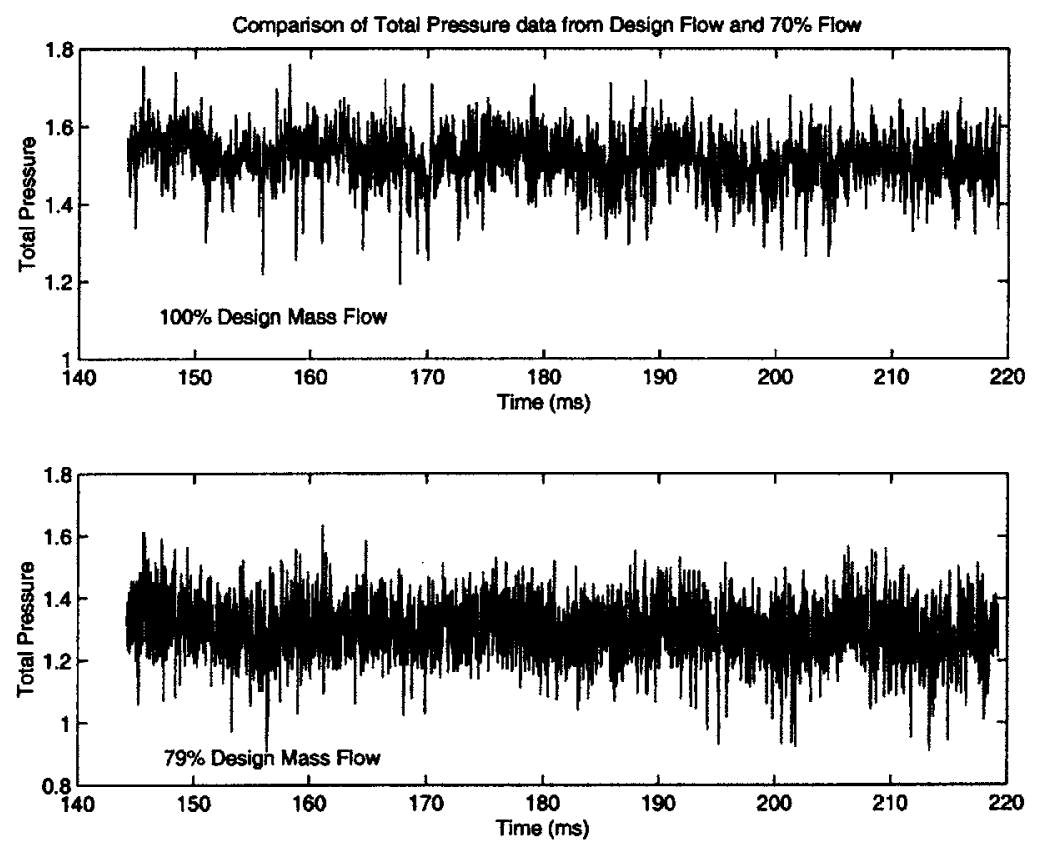

Figure 4-28: Un-averaged total pressure data at $100 \%$ and $79 \%$ Mass Flow 


\subsection{Parameter View of Off-design Performance}

In order to understand the off-design performance and seemingly large flow range, the offdesign performance of several important blade sections of the stage were investigated using a simple parameter study. This section presents the variation of stator Mach number, absolute flow angle, and stator diffusion factor for the hub and tip section of the stator at $100 \%$ and $79 \%$ design mass flow. From Merchant [14], the hub section of the stator is the most sensitive to flow disturbances.

Because the rotor has a negative exit relative flow angle near the hub (the main flow is turned beyond the axis in an impulse type design), a lower mass flow means the flow exits the rotor with less absolute tangential velocity than at design mass flow. The opposite is true for the rotor tip where the exit relative flow angle is positive. Therefore, as the mass flow is decreased, the loading on the stator hub should decrease while the loading at the stator tip should increase. Figures 4-29 and 4-30 suggest the effect that the lower mass flow can have on the local diffusion factor at the hub and tip sections. The diffusion factor decreases at the stator hub and increases at the stator tip.

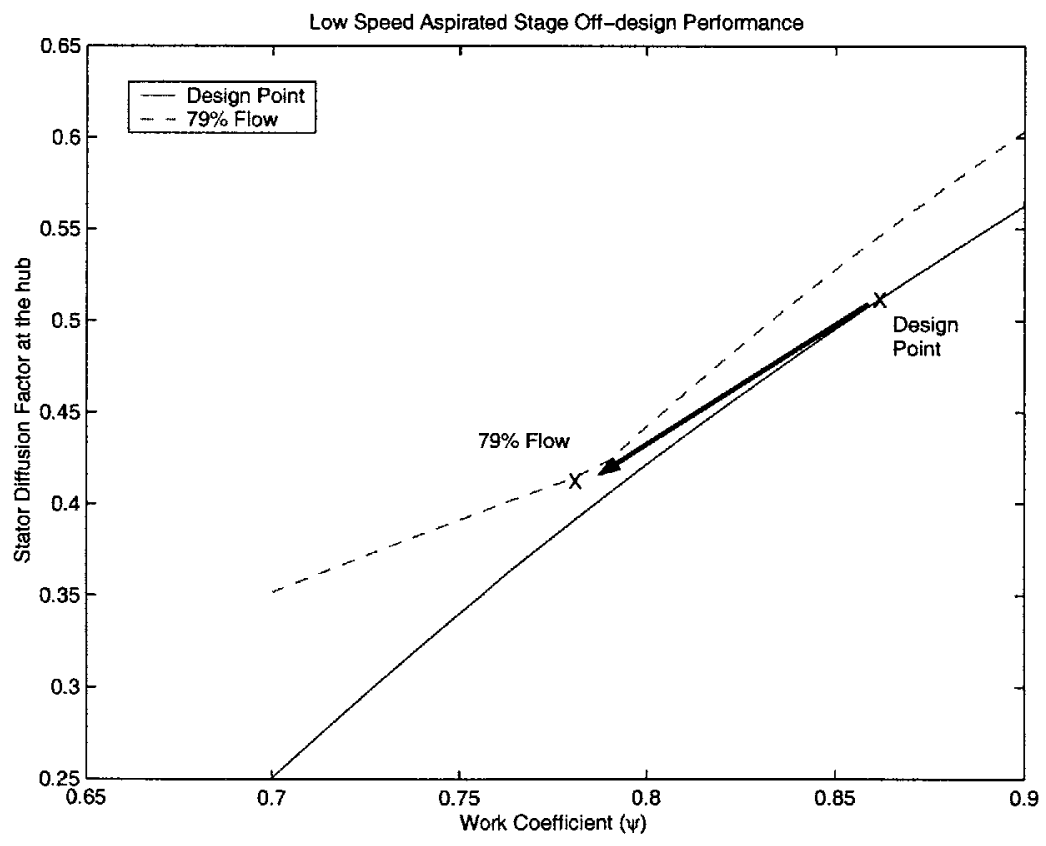

Figure 4-29: Stator hub section diffusion factor for $79 \%$ and $100 \%$ design mass flow

Figures 4-31 and 4-32 show the effect that the lower mass flow can have on the inlet 


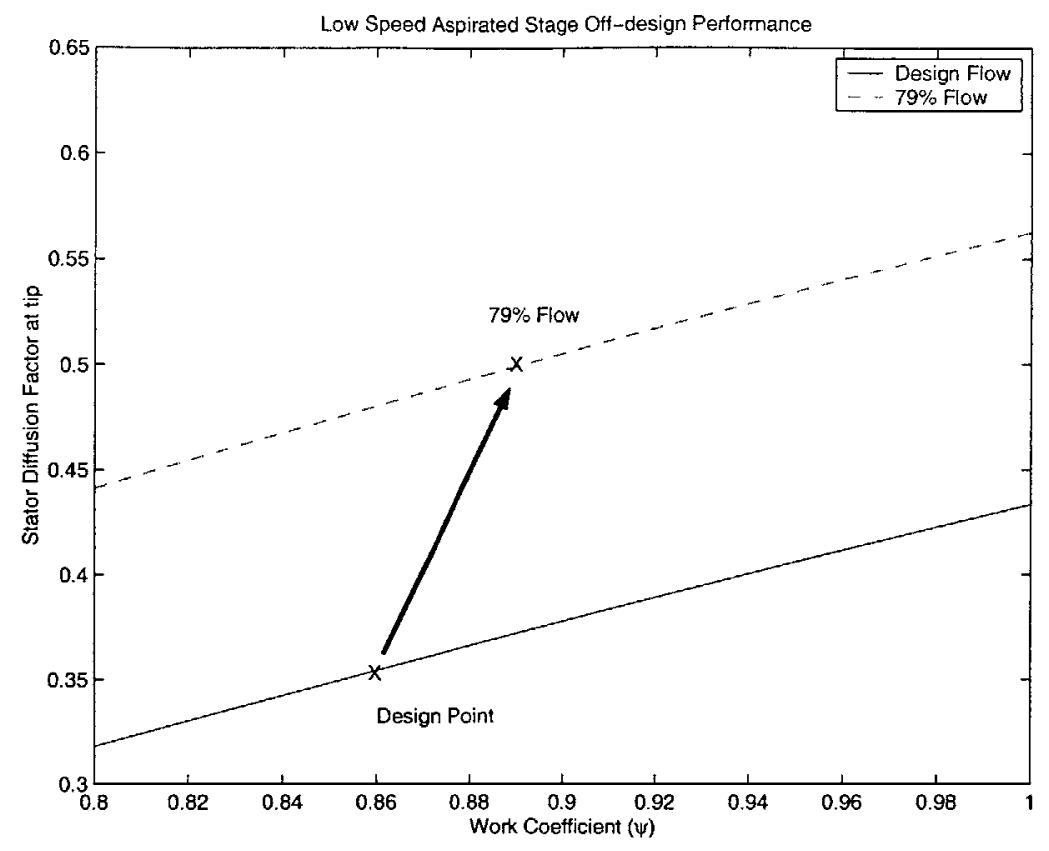

Figure 4-30: Stator tip section diffusion factor for $79 \%$ and $100 \%$ design mass flow

Mach number at the stator hub and tip sections. The hub section sees a decrease in stator inlet Mach number from 1.15 to .95 . This reduction significantly reduces the passage shock strength which, along with the aspiration, helps to keep the blade boundary layer from a large separation. The tip section also sees an decrease in the inlet Mach number from .92 to .82 .

Another important factor in the blade section performance is the incidence angle. Figures 4-33 and 4-34 show the change in inlet flow angle into the blade sections. The hub section shows an incidence increase of only 4 degrees while the tip section shows an incidence increase of 8 degrees.

The result of this parameter analysis is that at lower mass flows the stator tip section boundary layer is more likely to separate than the hub section because of the increase in diffusion factor and incidence. 


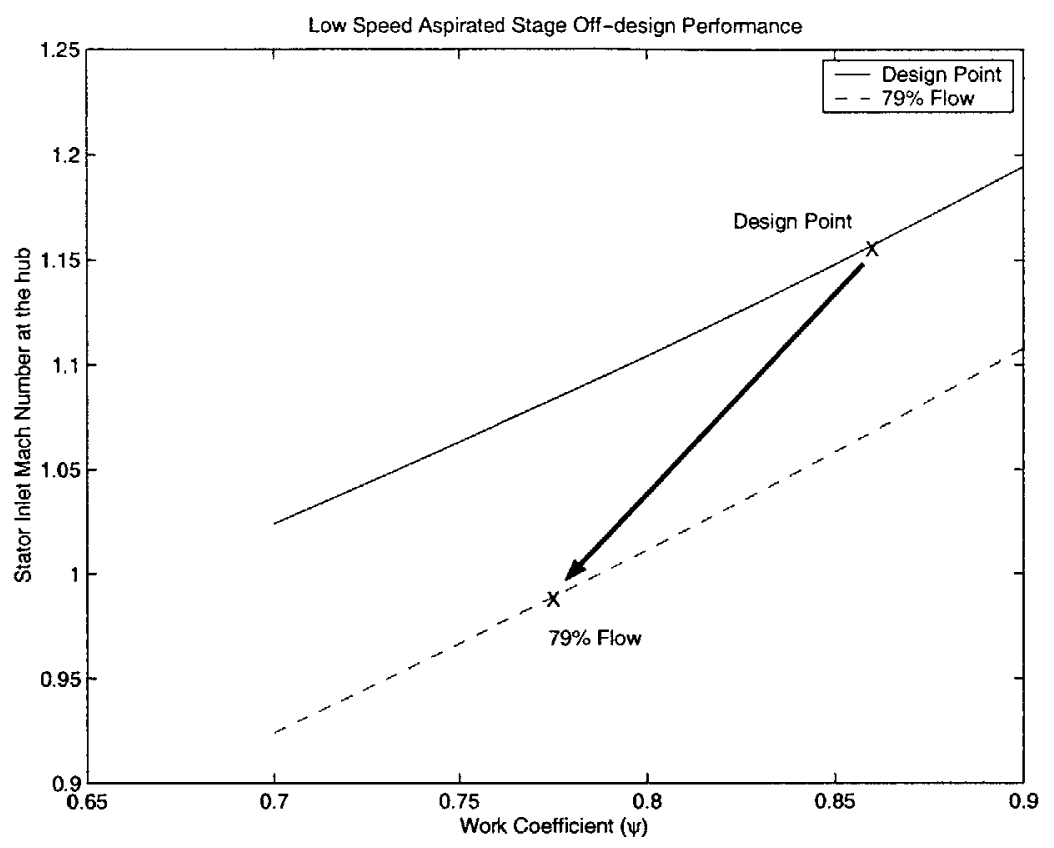

Figure 4-31: Stator hub section inlet Mach number for $79 \%$ and $100 \%$ design mass flow

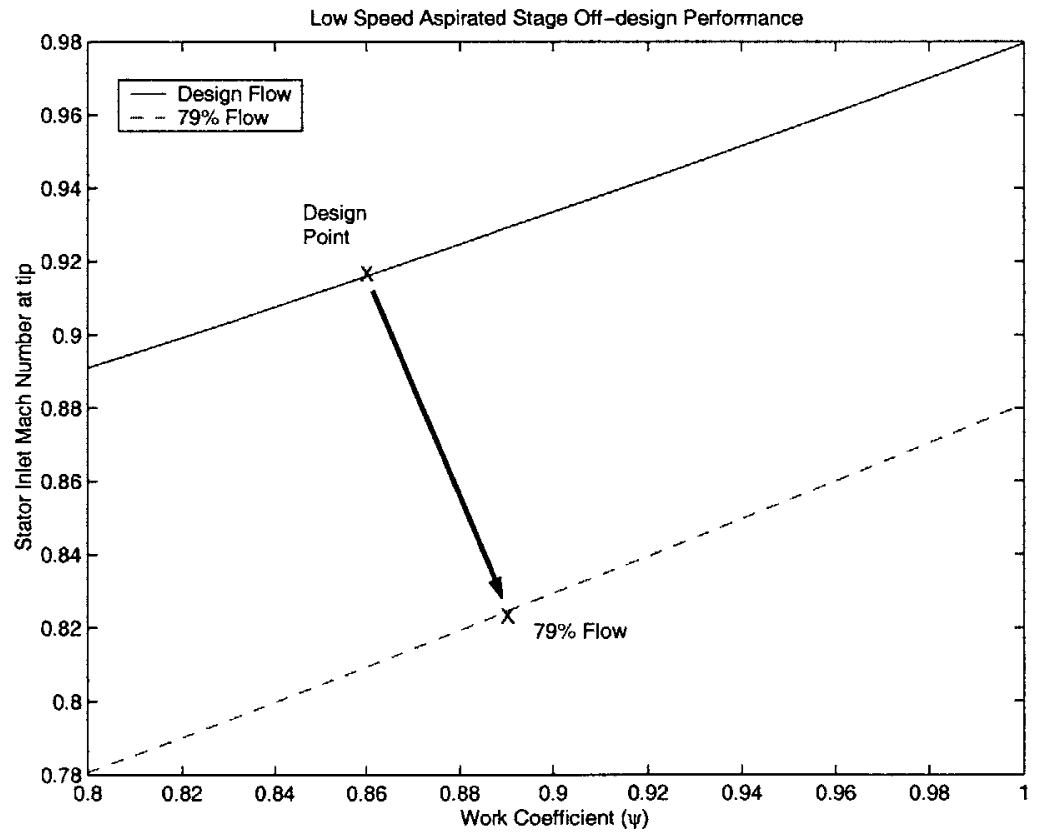

Figure 4-32: Stator tip section inlet Mach number for $79 \%$ and $100 \%$ design mass flow 


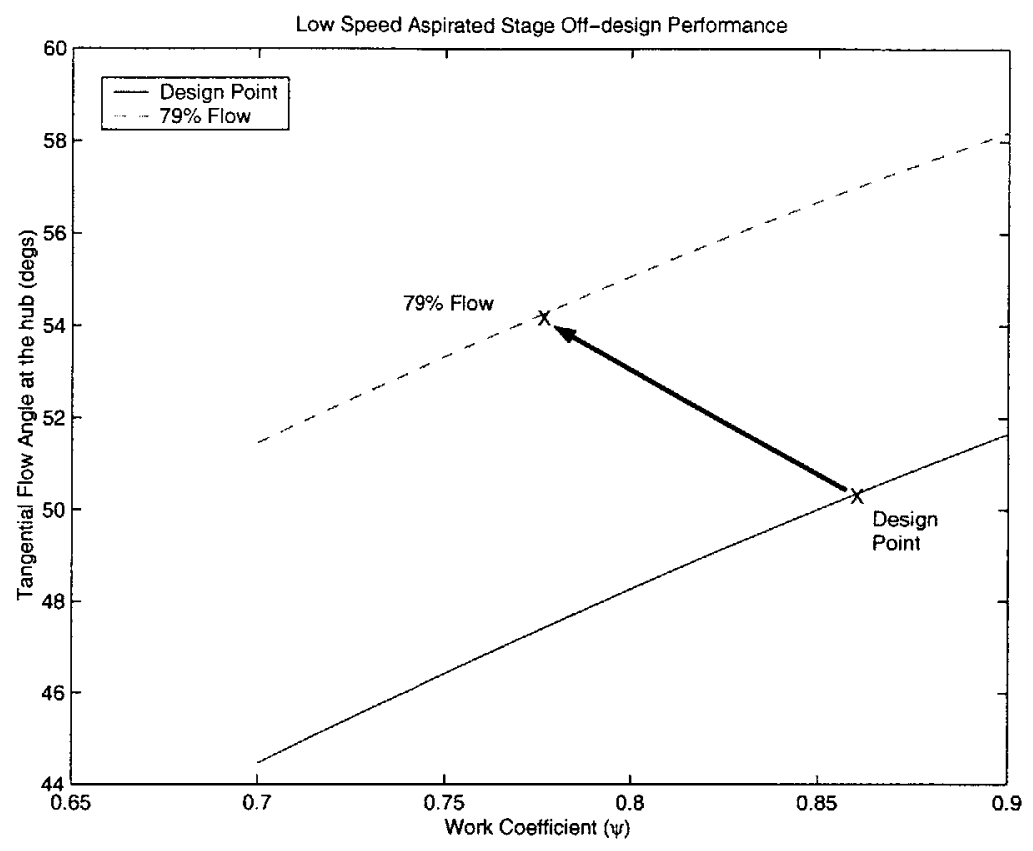

Figure 4-33: Stator hub inlet tangential flow angle for $79 \%$ and $100 \%$ design mass flow

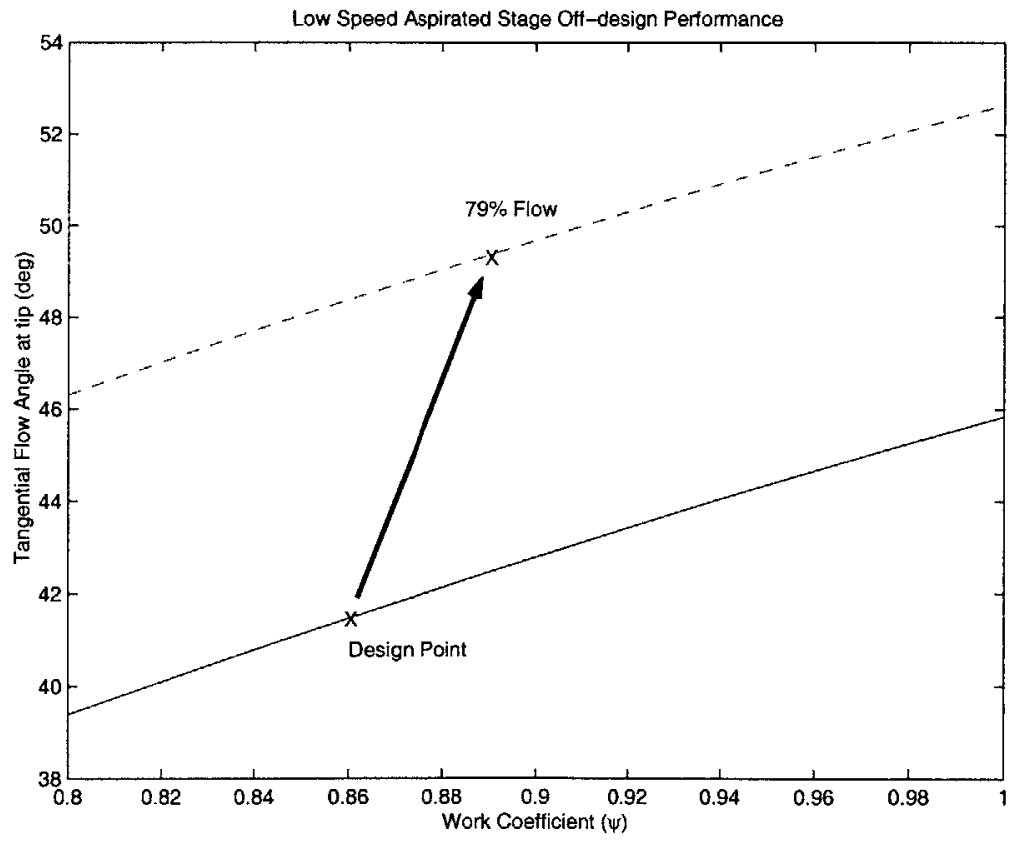

Figure 4-34: Stator hub inlet tangential flow angle for $79 \%$ and $100 \%$ design mass flow 


\subsection{Summary of Experimental Results}

Ensemble-averaged data is compared with the APNASA predictions at five different radial locations. Discrepancies between the pitchwise averaged data and the APNASA predictions exist in the spanwise distributions of total pressure and total temperature. The blade to blade variation of total pressure also seems to indicate a large amount of unsteadiness in the flowfield. If this unsteadiness is due to unsteady vortex shedding, then the discrepancy in spanwise total pressure and total temperature could be due to radial transport induced by the vortex shedding. The next chapter addresses the issue of unsteady vortex shedding, and its impact on the measured values of total pressure and total temperature compared to the APNASA predicted values.

The time-averaged total pressure downstream of the stator over a grid of points is compared to the APNASA prediction. The off-design behavior of the stage is investigated. A speedline corresponding to the $100 \%$ design speed was measured down to a mass flow of $79 \%$

flow. This speedline is compared to speedline predictions made by APNASA and a Pratt and Whitney 3-D viscous analysis. 


\section{Chapter 5}

\section{Unsteady Radial Transport}

\subsection{Introduction}

In the previous chapter, the discussion of experimental results and comparison to the APNASA results, have followed the practice of comparing the ensemble averaged data to the calculations, which are intrinsically periodic with blade spacing. But the data taken in the Blowdown Compressor contains additional information, in the form of the actual time dependent measurements taken with the 4 -way probe. These measurements show two phenomena which merit closer attention.

First, examination of the time traces of any flow property downstream of the rotor shows large fluctuations from blade to blade, so that the flow is in fact not even approximately periodic with blade passing. This unsteadiness in rotor coordinates, has been observed in the past ([3]) and its cause and consequences have been examined in detail ([4], [9]). It is due to the shedding of radial vortex streets in the wakes of the blades. The findings of these investigators will be applied to the present data set below.

The other phenomenon, which is believed to be in part at least, a consequence of the unsteadiness in rotor coordinates, or rather to the vortex shedding that causes it, is a measured efficiency greater than unity near the hub of the rotor. Measured efficiencies greater than unity have been reported for a number of rotors ([27], [2]). The measurement techniques that have yielded them have ranged from conventional temperature and stagnation pressure 
rakes to the MIT time resolved measurements such as are reported here.

One plausible explanation for these, seemingly anomolous, measured efficiencies, is radial transport and entropy segregation in the shed vortices. The transport processes produced by the vortices have been examined by Kotidis, and his methods will be employed here to examine this possibility. As we shall see, such transport does at least partially explain the radial variation of the efficiency of the rotor, which is higher at the hub and lower at the tip than the APNASA calculations predict.

These interesting flow phenomena seen in the experimental data require a more detailed investigation into the time-accurate performance of the stage. In this chapter, the time accurate data taken downstream of the rotor is fit to a 2-D unsteady vortex shedding model developed by Gertz [4] that was later extended to spanwise coherent vortex shedding by Kotidis [9]. Experimental evidence from [9] showed that flow is redistributed spanwise through the rotor and that the spanwise redistribution occurs mostly within the blade wakes. Extending the vortex model to include radial transport within the shed vortices showed that spanwise redistribution of flow could be explained by the radial flows induced within the vortex cores. Because the vortex shedding is an unsteady phenomenon superimposed on the rotor blade steady-state behavior, these spanwise flows are not predicted by standard steady computational solvers. By correcting the full, unsteady performance for these flows, the experimental data can be better compared to the APNASA solution which assumes steady flow.

\subsection{Vortex Shedding and 2-D Modelling of the Rotor Wakes}

Von Karman type vortices have been observed in the wakes of flat plates and other objects, both isolated and in cascade. Gertz [4] was the first to recognize regular arrays of vortex streets in the wakes of a transonic rotor in data gathered in the MIT Blowdown Compressor Facility. Using the Laser Anemometry (LA) measurements given in [22] and [16] by Strazisar and Powell that were gathered on the same compressor stage, a regular vortex 
street was evident through the bi-modal character of the velocity probability density distribution (PDD). Figures 5-1 and 5-2 show the experimental findings. Figure 5-1 shows the ensemble-averaged pitchwise velocity profile from the LA measurements. Figure 5-2 shows the probability density distribution of velocities measured at various locations within the pitch denoted by the given letter. Gertz was able to infer the vortex street properties from the experimental data. He modelled the vortex street as two staggered rectilinear rows of Rankine vortices of opposite sign located in a uniform free stream. The flow in the core is considered a solid body rotation with the flow outside the core being a potential vortex.

A complete description of the 2-D vortex fitting process is described by Gertz in [4]. A brief description of the modelling process is presented here to facilitate the understanding of the vortex model. Figure 5-3 from [4] is a schematic drawing of the simplified vortex street behind the rotor blade and shows the important parameters used in the model.

There are three basic parameters that are used to describe the vortex street, the coreradius ratio, $\frac{r_{0}}{h}$, the vortex strength, $k$, and the vortex spacing ratio,$\frac{h}{a}$. The core-radius ratio, $\frac{r_{0}}{h}$, and vortex strength, $k$, were chosen to match the average shape and depth of the wake profile measured by the LA. The spacing ratio, $\frac{h}{a}$, is then chosen so that the bimodal velocity distribution closely matches the velocities measured by the LA. This was accomplished by matching the upper and lower most probable velocities. A core-radius ratio of 0.5 was chosen so that the edges of the cores of both the upper and lower vortex rows correspond to the centerline of the wake. Therefore, $h$ is taken to be one half of the wake width. The final values of the vortex parameters are shown in table (5.1), where $U_{0}$ is the free stream velocity in the relative frame, and $U_{p}$ is the angular velocity at the edge of the core and equal to $\omega_{0} r_{0}$. Equation (5.1) relates the pressure defect velocity to the vortex strength.

\begin{tabular}{||ll|}
\hline Spacing Ratio $\left(\frac{h}{a}\right)$ & $=0.635$ \\
Core-radius Ratio $\left(\frac{r_{0}}{h}\right)$ & $=0.5$ \\
Vortex Strength $\left(\frac{k}{2 \pi a U_{0}}\right)$ & $=0.07$ \\
Pressure Defect Velocity Ratio $\left(\frac{U_{p}}{U_{0}}\right)$ & $=0.2205$ \\
\hline
\end{tabular}

Table 5.1: Vortex Street Parameters for the NASA LeRC Stage 67 


\section{MEAN ABSOLUTE VELOCITY DISTRIBUTION \\ MEASURED BY THE LASER ANEMOMETER}

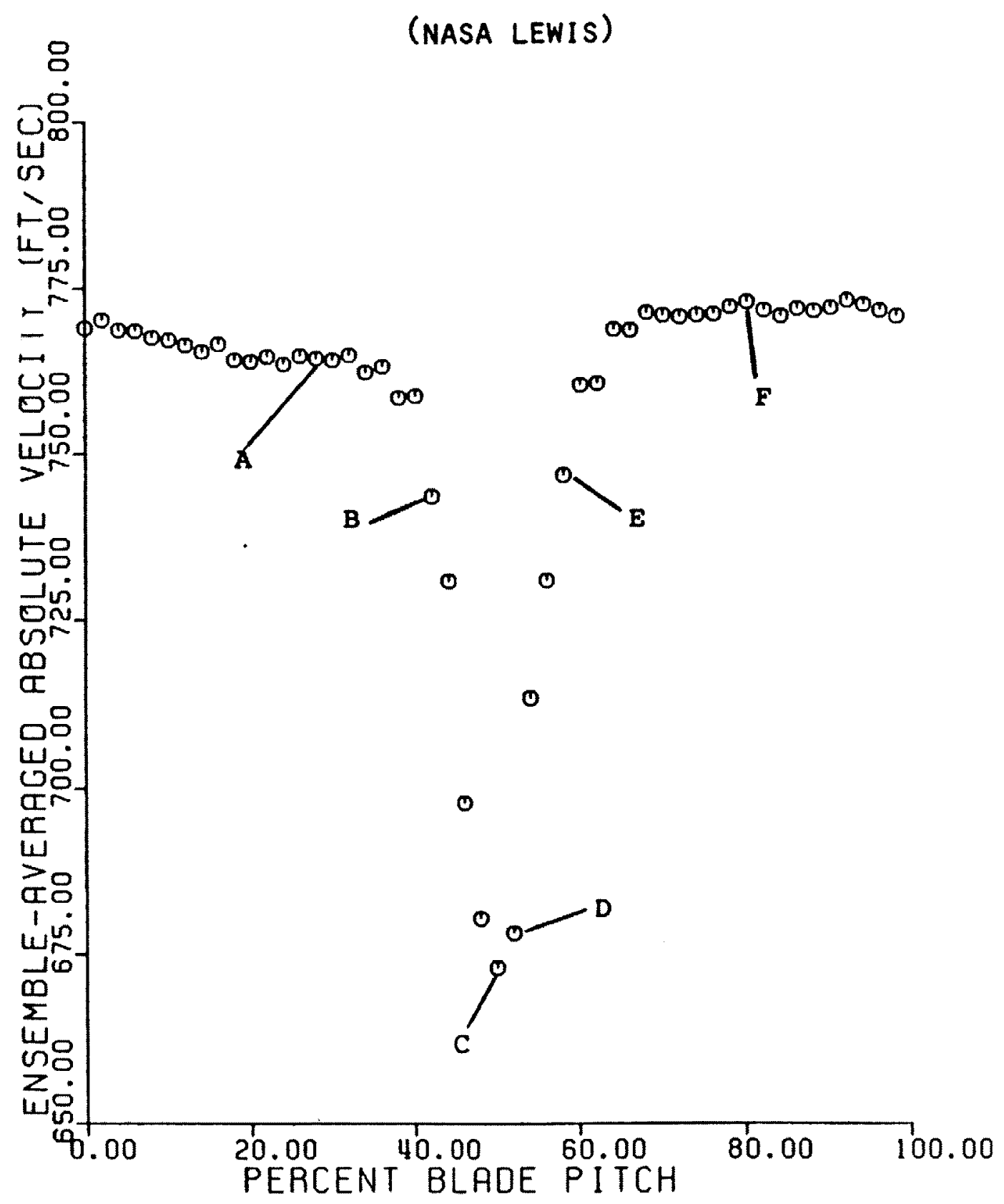

Figure 5-1: Ensemble-averaged wake profile from the LA measurements from [4] 

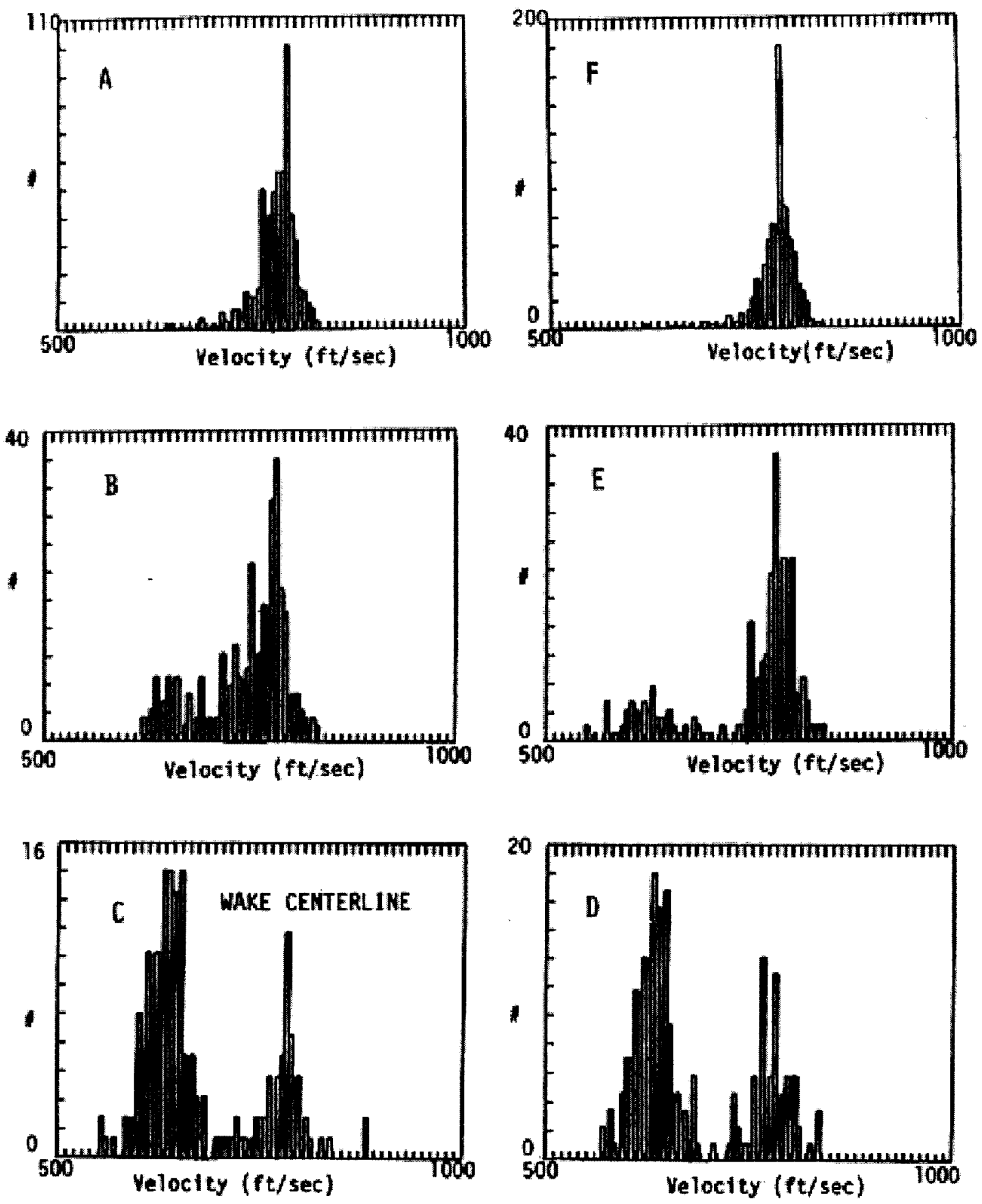

Figure 5-2: Velocity probability density for different points within the wake from [4] 


\section{GEOMETRY OF ROTOR BLADE VORTEX STREET}

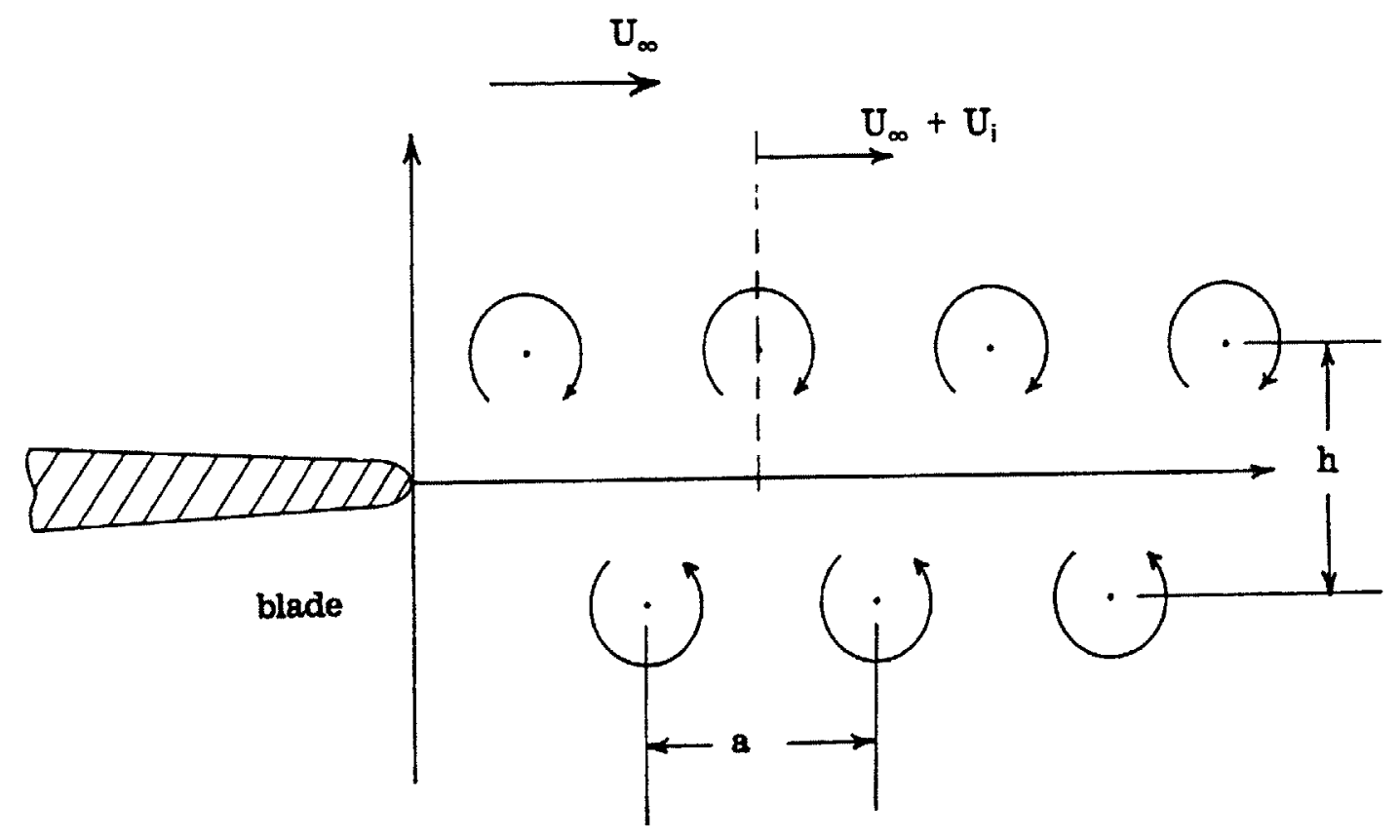

Figure 5-3: Schematic of the von Karmen vortex street model used by Gertz [4]

$$
U_{p}=\frac{k}{2 \pi r_{0}}
$$

Once the vortex parameters are determined, the velocity and pressure fields are fully determined and a shedding frequency can be calculated. For the NASA rotor, a shedding frequency of $15.9 \mathrm{kHz}$ was found for that particular span location. 


\subsection{Fitting the Vortex Parameters to Experimental Data}

Because the four-way probe measures total and static pressure, radial flow angle, and tangential flow angle, the vortex parameters could be fit directly to the measured Mach numbers in a manner similar to Gertz [4]. The time accurate measurements of Mach number across the pitch produced complete distributions of velocity for each blade passage. Free stream flow values of relative flow angle, relative Mach number, and wake-to-pitch ratio necessary for the fitting are taken as the ensemble-averaged values. The wake depth is also taken as the ensemble-averaged value.

The vortex fitting begins with the shape of the wake. The ensemble-averaged wakes measured in the experiment show the same shape as the wake given by Gertz [4] therefore the same value of core radius ratio is used for all the spanwise locations, $\frac{r_{0}}{h}=0.5$. This radius ratio sets the edge of the core of the upper and lower vortices to be equal to the edge of the wake. With the core size determined, now the wake depth is used to determine the vortex strength. The vortex strength is picked to match the depth of the ensemble-averaged wake. Next the time-accurate wake data is used to determine the vortex spacing ratio, $\frac{h}{a}$. Like the method used by Gertz [4], the results of the time accurate measurements are grouped into velocity 'buckets' to determine the most probable velocities along the wake centerline. Then the spacing ratio is varied until a good agreement is reached between the model and the data. Table 5.2 shows the values of the vortex parameters for each spanwise location.

\begin{tabular}{||l|c|c|c|c|c|}
\hline Radius Ratio $\left(\frac{r}{r_{t} i p}\right)$ & .77 & .83 & .89 & .94 & .97 \\
\hline Spacing Ratio $\left(\frac{h}{a}\right)$ & .105 & .155 & .215 & .325 & .250 \\
Core-radius Ratio $\left(\frac{r_{0}}{h}\right)$ & 0.5 & 0.5 & 0.5 & 0.5 & 0.5 \\
Vortex Strength $\left(\frac{2 \pi}{2 \pi a U_{0}}\right)$ & .016 & .028 & .021 & .036 & .055 \\
\hline
\end{tabular}

Table 5.2: Vortex Street Parameters for the Aspirated Fan Stage 


\subsection{Extension to Spanwise Vortex Shedding}

In order to include spanwise flows, the 2-D vortex shedding model must be applied at several different radial locations. After fitting the vortex parameters to the data, the static pressure field is calculated. Following Gertz [4], the pressure coefficient is defined in equation (5.2), where the subscript 0 denotes the free stream conditions and $U_{p}$ is the pressure defect velocity.

$$
C_{p}=\frac{P-P_{0}}{\rho_{0} U_{p}^{2}}
$$

Now the wake can be broken into two separate regions. The solid body vortex core region and the potential vortex region outside the core. From Gertz [4], the pressure coefficient for the potential vortex region is given in equation (5.3), where $\bar{q}^{\prime}$ is the total velocity in the coordinate frame moving with the vortex street and $\bar{U}_{i}$ is the induced velocity. Both are normalized by the pressure defect velocity. Each vortex row induces a motion in the opposite row which results in a motion of the entire street. The induced velocity is found by equation (5.4) from Gertz [4].

$$
\begin{gathered}
C_{p}=-\frac{1}{2} \bar{q}^{2}-\frac{1}{2} \bar{U}_{i}^{2} \\
U_{i}=\frac{-k}{2 a} \tanh \frac{\pi h}{a}
\end{gathered}
$$

For the pressure distribution in the vortex cores, Gertz [4] uses equation (5.5) where $r$ is the distance from the point to the core center, $r_{0}$ is the radius of the vortex core, and $\left\langle\bar{q}^{2}\right\rangle$ is the average of the velocity squared around the vortex core at the core radius, $r=r_{0}$. For values of $r<r_{0}, q_{0}$ is evaluated at a point on the circumference $\left(r=r_{0}\right)$ where a ray from the center of the vortex through the point in question intersects the edge of the vortex core at $r=r_{0}$.

$$
C_{p}=-\frac{1}{2} \bar{q}_{0}^{2}\left[2-\left(\frac{r}{r_{0}}\right)^{2}\left(2-\frac{\bar{q}_{0}^{2}}{<\bar{q}_{0}^{2}>}\right)\right]+\frac{1}{2} \bar{U}_{i}^{2}
$$

From equations (5.3) and (5.5), the static pressure distribution within the wake is calcu- 
lated for each radial location. Using these static pressure distributions, the spanwise flows can be estimated using the approach of Kotidis [9]. A more detailed description of the derivation of the spanwise flow equations can be found in [9]. A brief description of the derivation is given here. Using the frame of reference rotating with the blade row, equations (5.6) and (5.7) show the equation of motion in vector form for the wake flow and the free stream flow, where $\vec{w}=\left(w_{r}, w_{\theta}, w_{z}\right)$ is the relative velocity vector for the flow in the wake and $\vec{W}=\left(W_{r}, W_{\theta}, W_{z}\right)$ is the relative velocity vector for the potential core flow.

$$
\begin{aligned}
& \frac{D \vec{w}}{D t}+2 \vec{\omega} \times \vec{w}=\omega^{2} \vec{r}-\frac{\nabla p}{\rho_{w a k e}} \\
& \frac{D \vec{W}}{D t}+2 \vec{\omega} \times \vec{W}=\omega^{2} \vec{r}-\frac{\nabla P}{\rho_{0}}
\end{aligned}
$$

Subtracting equation (5.6) from equation (5.7) eliminates the centrifugal force term. The radial component of the combined equation gives equation (5.8), where $\Delta t$ is the convection time from the rotor trailing edge to the probe location.

$$
\Delta w_{r}=-\Delta t\left[2 \omega\left(W_{\theta}-w_{\theta}\right)-\frac{1}{\rho_{0}} \frac{\partial P}{\partial r}+\frac{1}{\rho_{\text {wake }}} \frac{\partial p}{\partial r}\right]
$$

This equation is solved at each radial location and at each point in the wake giving the radial distribution of spanwise velocity with the simplifying assumption that the convection time is nearly constant throughout the wake. Therefore, $\Delta t$ is equal to the convection time of the vortex core from the rotor trailing edge to the probe. Given the density, these spanwise velocities are used to calculate the spanwise mass flows within the wake. At this point, this calculation differs from Kotidis [9]. This calculation includes the spanwise flows throughout the wake, while Kotidis only considered flows within the vortex core and ignored induced spanwise flows in the remainder of the wake. The flows outside the vortex cores have a significant effect on the amount of mass that can be exchanged between streamtubes.

The result of these calculations is that the radial velocities within the wake are roughly constant from the $28 \%$ span location to the $84 \%$ span location with the spanwise flow directed toward the tip region. The $92 \%$ span location shows both positive and negative velocities 
indicating that there is flow both into and out of the region. Table 5.3 show the calculated values of spanwise flow between regions normalized by the total inlet mass flow. The flows are presented in matrix form where the column number corresponds to the region of flow injection, and the row number corresponds to the region of flow removal. For example, the value in column 2, row 1 represents spanwise flow from region 1 into region 2 .

\begin{tabular}{||c|c|c|c|c|c|}
\hline Region & 1 & 2 & 3 & 4 & 5 \\
\hline \hline 1 & .000 & .004 & .000 & .000 & .000 \\
\hline 2 & .018 & .000 & .000 & .000 & .000 \\
\hline 3 & .000 & .020 & .000 & .000 & .000 \\
\hline 4 & .000 & .000 & .011 & .000 & .000 \\
\hline 5 & .000 & .000 & .000 & .008 & .000 \\
\hline
\end{tabular}

Table 5.3: Spanwise flow values

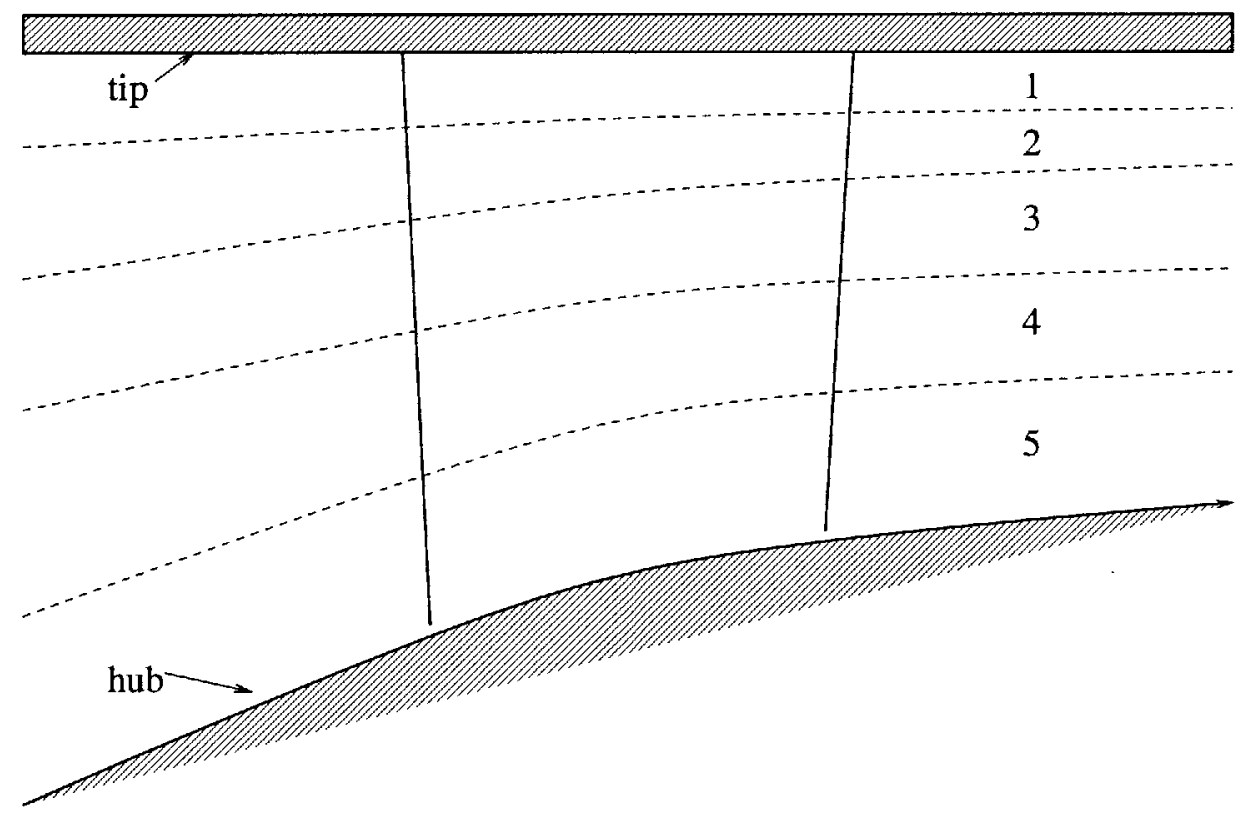

Figure 5-4: Schematic of the flow regions used in the calculation of spanwise redistribution of flow properties

These radial velocities in the wake are supported by the experiment. Time accurate measurements of radial velocity are shown in figures 5-5, 5-7, and 5-9. Figures 5-6, 5-8, and 5-10 show the present the APNASA predictions of radial velocity at different spanwise locations. Positive radial Mach number corresponds to flow towards the tip region. 
The results from the APNASA analysis show a very small amount of radial transport within the wake compared to the measurements made in the experiment. Figures 5-5 shows radial Mach numbers varying from -0.2 to 0.2 in the wake for the experimental data. Figure 5-6 shows only a variation of 0.01 to 0.04 in wake region for the APNASA analysis. The measurements at other radial locations also show large variation in radial Mach number in the wake compared to the APNASA solutions. These comparison plots show that the APNASA analysis does not accurately predict the radial flows within the wake. These radial flows redistribute flow properties which can have a large influence on the spanwise variation of total pressure and efficiency.

The vortex shedding model predicts flow into region 1 and out of region 1 corresponding to both positive and negative radial velocities. Figure 5-5 shows both positive and negative radial velocities in the wake corresponding to wake flow into and out of the region. For the flow in region 3, the vortex model only predicts flow towards the tip while figure 5-7 shows radial flows toward the hub and tip. Figure 5-9 shows some radial flow towards the tip but most radial flow towards the hub. The vortex model predicts a small radial flow towards the tip. Although the vortex shedding model does not exactly predict all the radial flows shown in the experimental data, its use does allow the data to be more closely compared with the APNASA solution. 


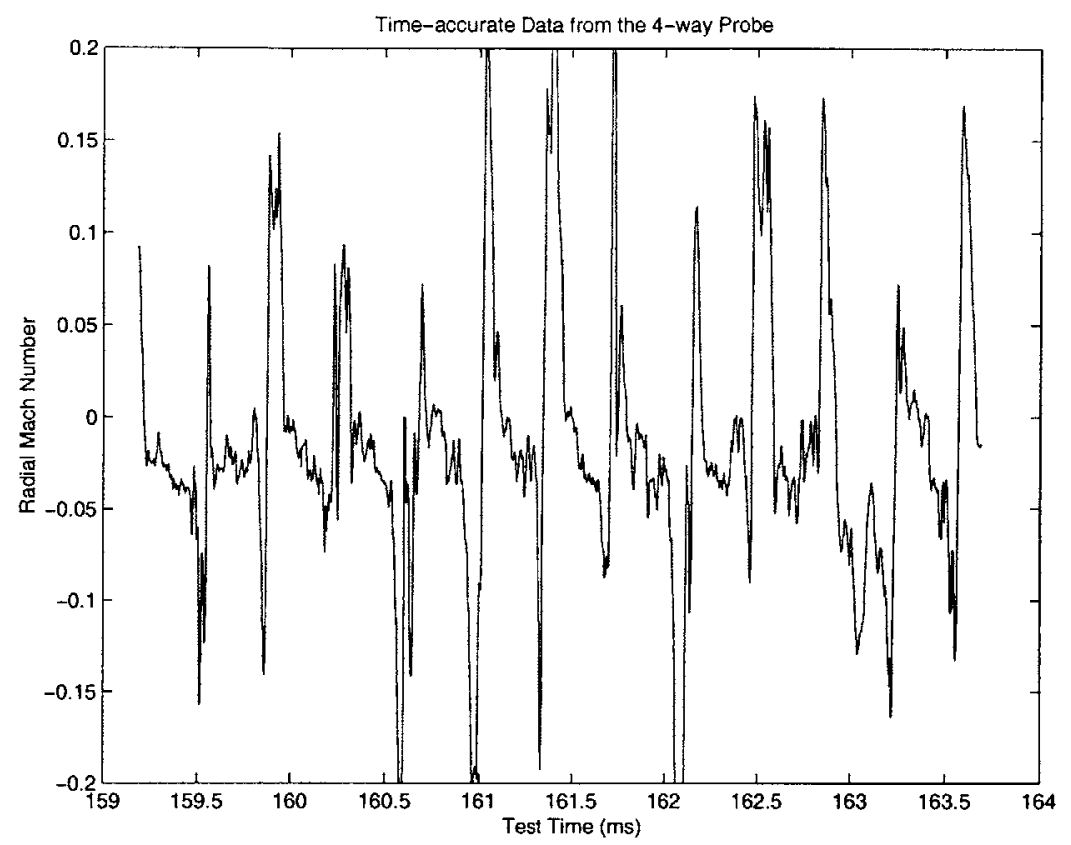

Figure 5-5: Time-accurate radial Mach number distribution at $92 \%$ span

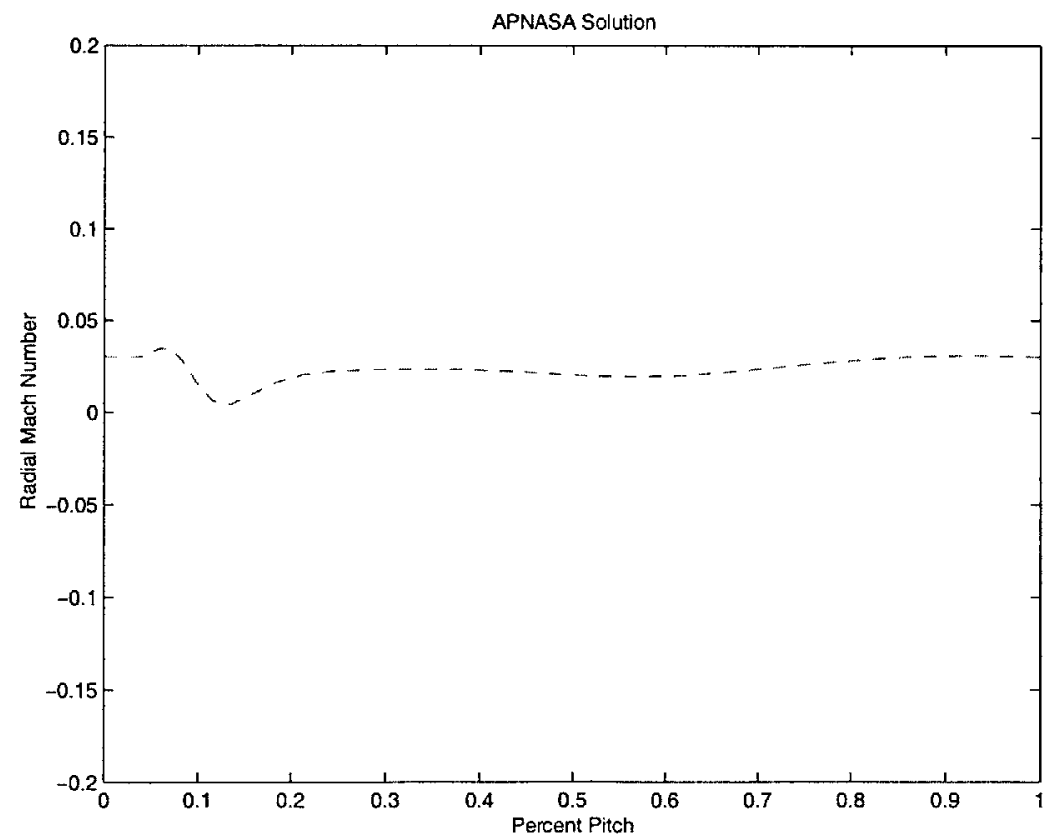

Figure 5-6: Radial Mach number distribution from APNASA at $92 \%$ span 


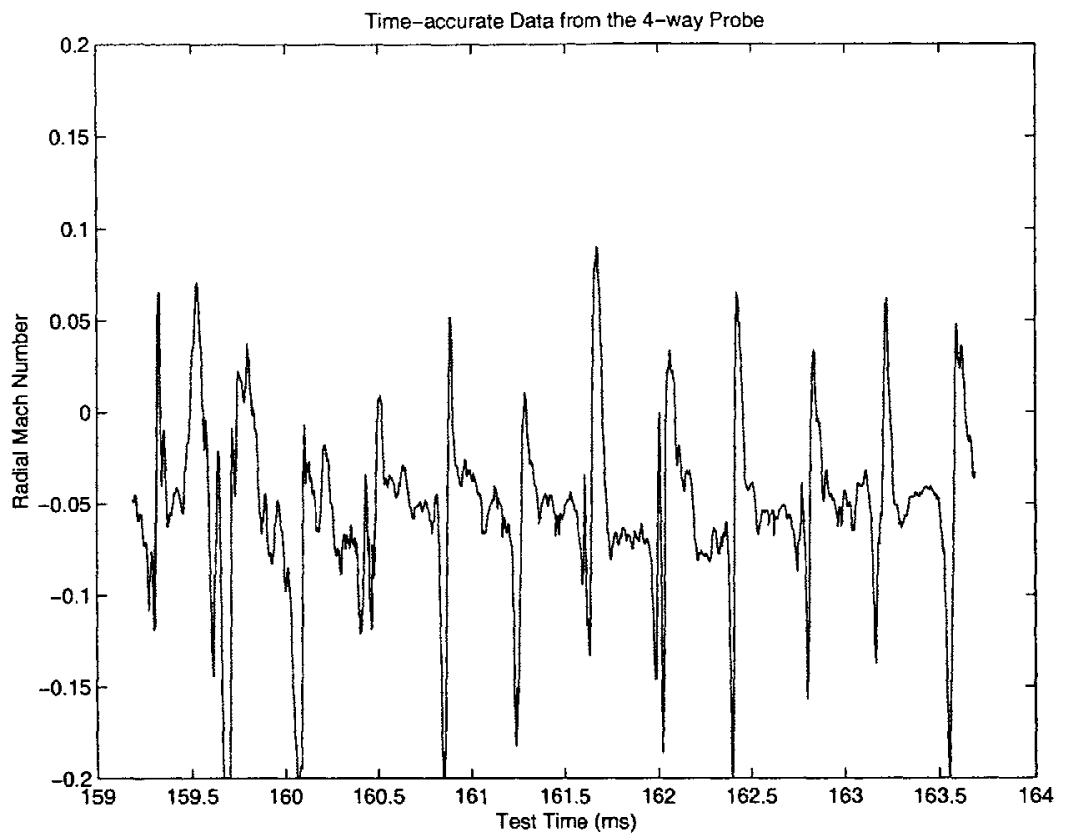

Figure 5-7: Time-accurate radial Mach number distribution at $65 \%$ span

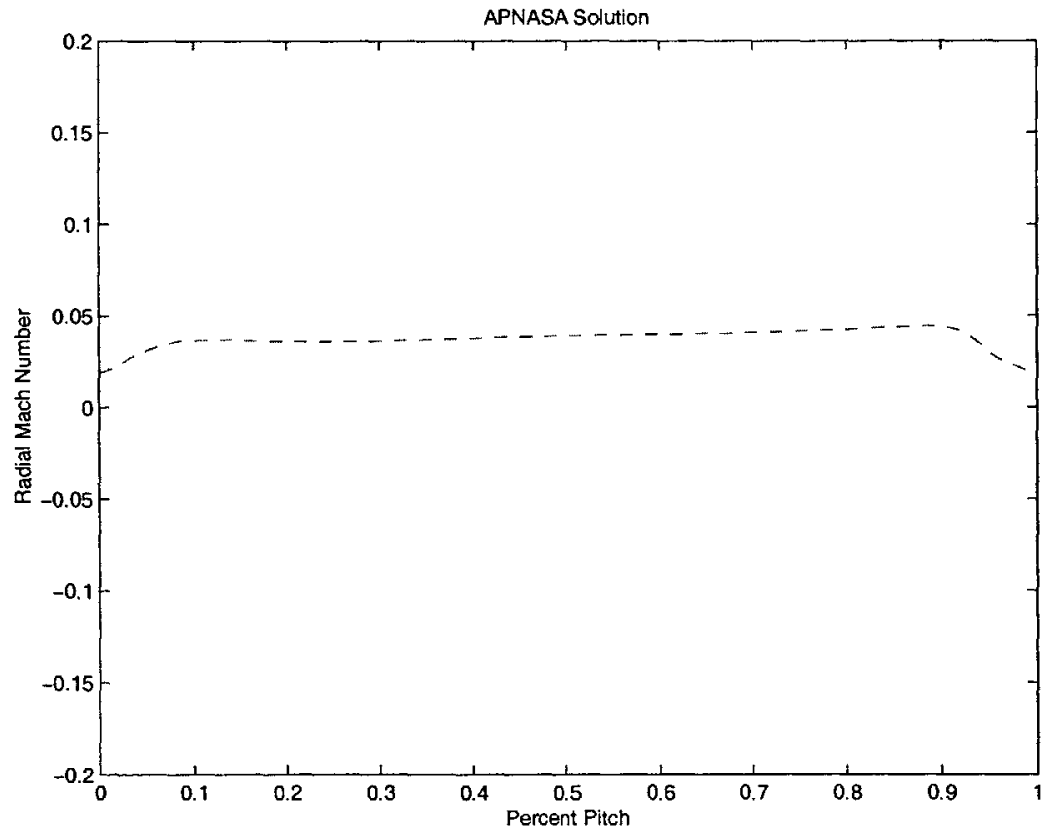

Figure 5-8: Radial Mach number distribution from APNASA at 65\% span 


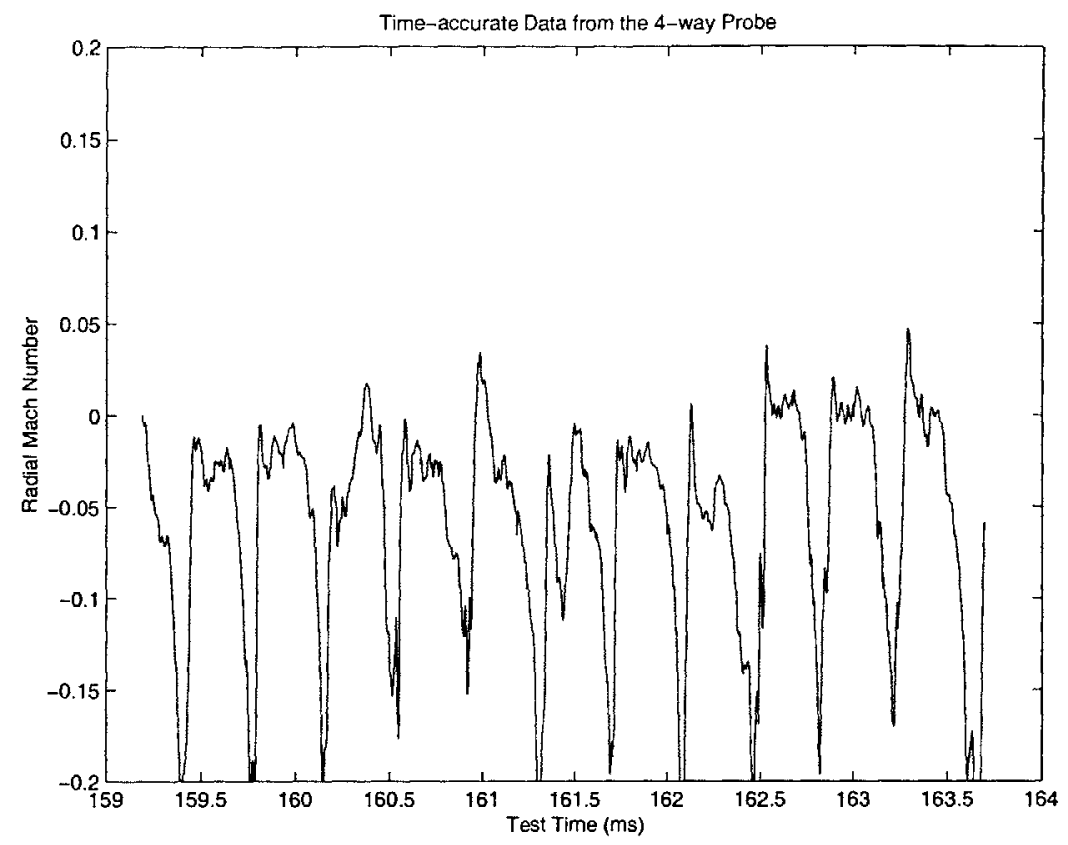

Figure 5-9: Time-accurate radial Mach number distribution at 28\% span

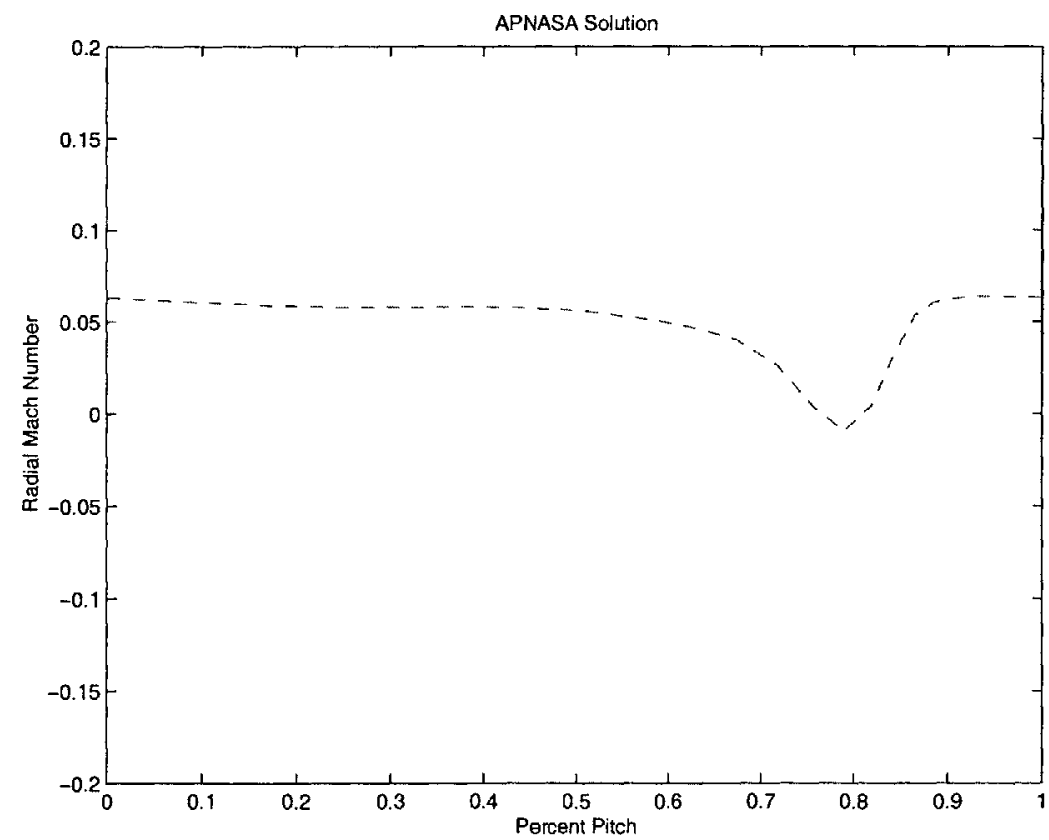

Figure 5-10: Radial Mach number distribution from APNASA at $28 \%$ span 


\subsection{Spanwise Redistribution of Total Pressure and To- tal Temperature}

Now that the amount of spanwise mass redistribution has been calculated from the vortex shedding model, the effects of the mass redistribution on the measured values of total pressure and total temperature must be estimated. The experimental results of Kotidis [9] show that most of the spanwise migration occurs in the blade wakes. Therefore, it is assumed that only the wake fluid moves in the spanwise direction. Kotidis presents a series of equations for the simple redistribution of flow properties downstream of the rotor without mixing effects. This idea of redistribution of flow properties is then extended to include complete mixing between the streamtube wakes. These two results (with and without mixing) should put a bound on the actual effect of spanwise flows on the measured flow properties. In this application of the work by Kotidis, the rotor exit is divided into annular regions as shown in figure 5-4. Spanwise flow is exchanged between the regions.

\subsubsection{No Spanwise Mixing}

In the description by Kotidis [9], the flow properties used in the redistribution process are total temperature and entropy. Kotidis [9] idealizes the flowfield downstream of the rotor as shown in figure 5-11. There is a free stream region and a wake region. Two factors $\lambda$ and $\kappa$ relate the total temperature and entropy in the wake region to the free stream region. The values of these variables come from the experimental data and vary in the spanwise direction.

A simple total temperature and entropy accounting argument is used to achieve equations (5.10) and (5.11), shown for region 1 only. In these equations, $\dot{\mu}_{i j}$ denotes the percent total inlet flow from region $i$ into region $j$. For example, $\dot{\mu}_{12}$ represents the percent of total inlet mass flow redistributed from region 1 into region 2 . Also, in these equations, the plus $(+)$ superscripts represent the flow property after fluid migration, and the minus (-) superscripts represent the flow property before fluid migration. In this case, the flow properties with the plus $(+)$ superscripts represent the experimental measured quantities. The flow properties 


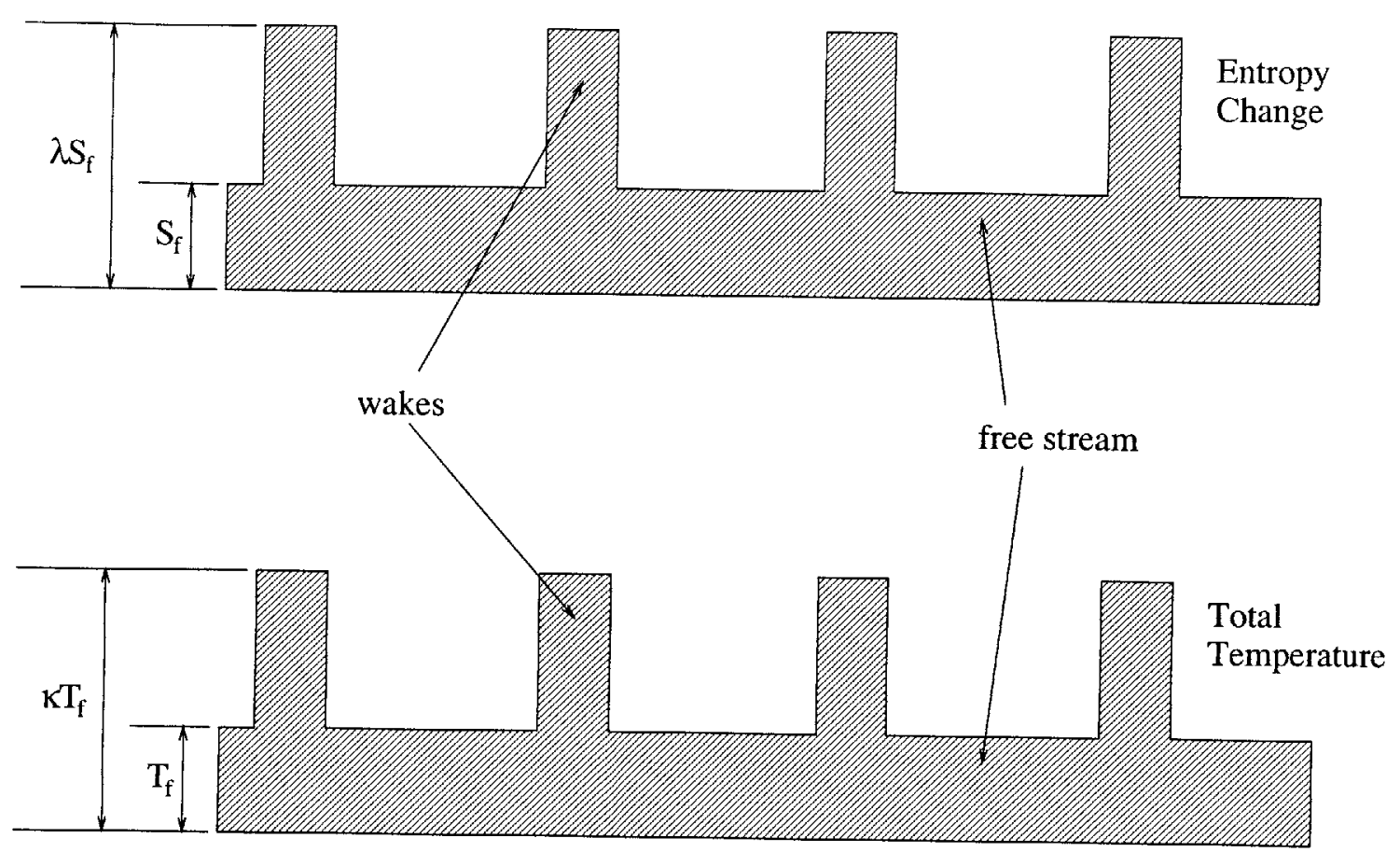

Figure 5-11: Idealized view of the pitchwise flow properties at the rotor exit

with the minus (-) superscripts represent the quantities corrected for spanwise transfer and are the unknowns in this set of equations.

$$
\begin{aligned}
& \left(\dot{m}_{1}+\dot{\mu}_{21}+\dot{\mu}_{31}+\dot{\mu}_{41}+\dot{\mu}_{51}-\dot{\mu}_{12}-\dot{\mu}_{13}-\dot{\mu}_{14}-\dot{\mu}_{15}\right) T_{1}^{(+)}= \\
& \quad\left(\dot{\mu}_{w 1} \kappa_{1} T_{f 1}^{(-)}+\dot{\mu}_{f 1} T_{f 1}^{(-)}\right)+\dot{\mu}_{21} \kappa_{2} T_{f 2}^{(-)}+\dot{\mu}_{31} \kappa_{3} T_{f 3}^{(-)}+\dot{\mu}_{41} \kappa_{4} T_{f 4}^{(-)}+ \\
& \quad \dot{\mu}_{51} \kappa_{5} T_{f 5}^{(-)}-\left(\dot{\mu}_{12}+\dot{\mu}_{13}+\dot{\mu}_{14}+\dot{\mu}_{15}\right) \kappa_{1} T_{f 1}^{(-)}
\end{aligned}
$$

$$
\begin{aligned}
& \left(\dot{m}_{1}+\dot{\mu}_{21}+\dot{\mu}_{31}+\dot{\mu}_{41}+\dot{\mu}_{51}-\dot{\mu}_{12}-\dot{\mu}_{13}-\dot{\mu}_{14}-\dot{\mu}_{15}\right) s_{1}^{(+)}= \\
& \left(\dot{\mu}_{w 1} \lambda_{1} s_{f 1}^{(-)}+\dot{\mu}_{f 1} s_{f 1}^{(-)}\right)+\dot{\mu}_{21} \lambda_{2} s_{f 2}^{(-)}+\dot{\mu}_{31} \lambda_{3} s_{f 3}^{(-)}+\dot{\mu}_{41} \lambda_{4} s_{f 4}^{(-)}+ \\
& \dot{\mu}_{51} \lambda_{5} s_{f 5}^{(-)}-\left(\dot{\mu}_{12}+\dot{\mu}_{13}+\dot{\mu}_{14}+\dot{\mu}_{15}\right) \lambda_{1} s_{f 1}^{(-)}
\end{aligned}
$$

Equations (5.10) and (5.11) are the equations for the first region. Similar equations 
can be written for each of the flow regions show in figure 5-4. Once the "no spanwise transfer' total temperature and entropy distributions are calculated then equation (5.12) is used to determine the spanwise efficiency distribution, where $\eta^{(-)}$is the 'no spanwise transfer' efficiency and $\tau^{(-)}$equals the 'no spanwise transfer' total temperature ratio defined in equation (5.11).

$$
\begin{gathered}
\tau_{1}^{(-)}=\frac{T_{1}^{(-)}}{T_{i n, 1}} \\
\eta^{(-)}=\frac{\tau^{(-)} e^{-\frac{s^{(-)}-s_{i n}}{C_{p}}}-1}{\tau-1}
\end{gathered}
$$

\subsubsection{With Spanwise Mixing}

In an extension of the method proposed by Kotidis [9], mixing of the wake fluid between the two streams is allowed. In the mixing case, the flow properties of choice are total temperature and total pressure. The 'no spanwise transfer' total temperature is calculated from the same set of equations found in section 5.5.1. The total pressure change is modelled as a constant area mixing process with injection and removal of mass. Using the influence coefficients from Shapiro [20], equation (5.13) gives the 'no spanwise transfer' total pressure, where the plus (+) and minus (-) superscripts again correspond to 'with spanwise transfer' and 'no spanwise transfer' respectively, $\dot{\mu}_{i n}$ is the mass flow into the region, $\dot{\mu}_{\text {out }}$ is the mass flow out of the region, $\dot{\mu}_{w 1}$ is the mass flow in the wake in region 1 , and $M_{m i x, 1}$ is the Mach number at which the mixing occurs.

$$
\frac{P_{w 1}^{(+)}-P_{w 1}^{(-)}}{P_{w 1}^{(-)}}=-\gamma M_{m i x, 1}^{2}\left(\frac{\dot{\mu}_{i n}-\dot{\mu}_{o u t}}{\dot{\mu}_{w 1}}\right)
$$

Using this estimate of the total pressure losses due to mixing in the wake, the 'no spanwise transfer' total pressure for that region can be found by mass averaging the new wake total pressure and the free stream total pressure. The 'no spanwise transfer' total pressure ratio for region 1 is defined by equation (5.14). 


$$
\pi_{1}^{(-)}=\frac{P_{1}^{(-)}}{P_{i n, 1}}
$$

Now with the 'no spanwise transfer' total temperature ratio from equation (5.11) and the 'no spanwise transfer' total pressure ratio from equation (5.14), then the spanwise efficiency distribution can be calculated using equation (5.15).

$$
\eta_{1}^{(-)}=\frac{\left(\pi_{1}^{(-)}\right)^{\frac{\gamma-1}{\gamma}}-1}{\tau_{1}^{(-)}-1}
$$




\subsection{Results of Spanwise Redistributions with and with- out Mixing}

The results of adjusting the flow properties downstream of the rotor to remove the effects of transport are shown in figure 5-12. In this figure, the measured experimental efficiency with the experimental error bars is shown along with the predicted efficiency distribution from the APNASA calculation. The two adjusted efficiency distributions are also shown, one which includes mixing losses and one that only redistributes the flow properties. The 'no mixing' distribution shows very little change from the measured values. The 'mixed' efficiency distribution shows a more uniform profile and approaches the efficiency profile predicted by the APNASA solution.

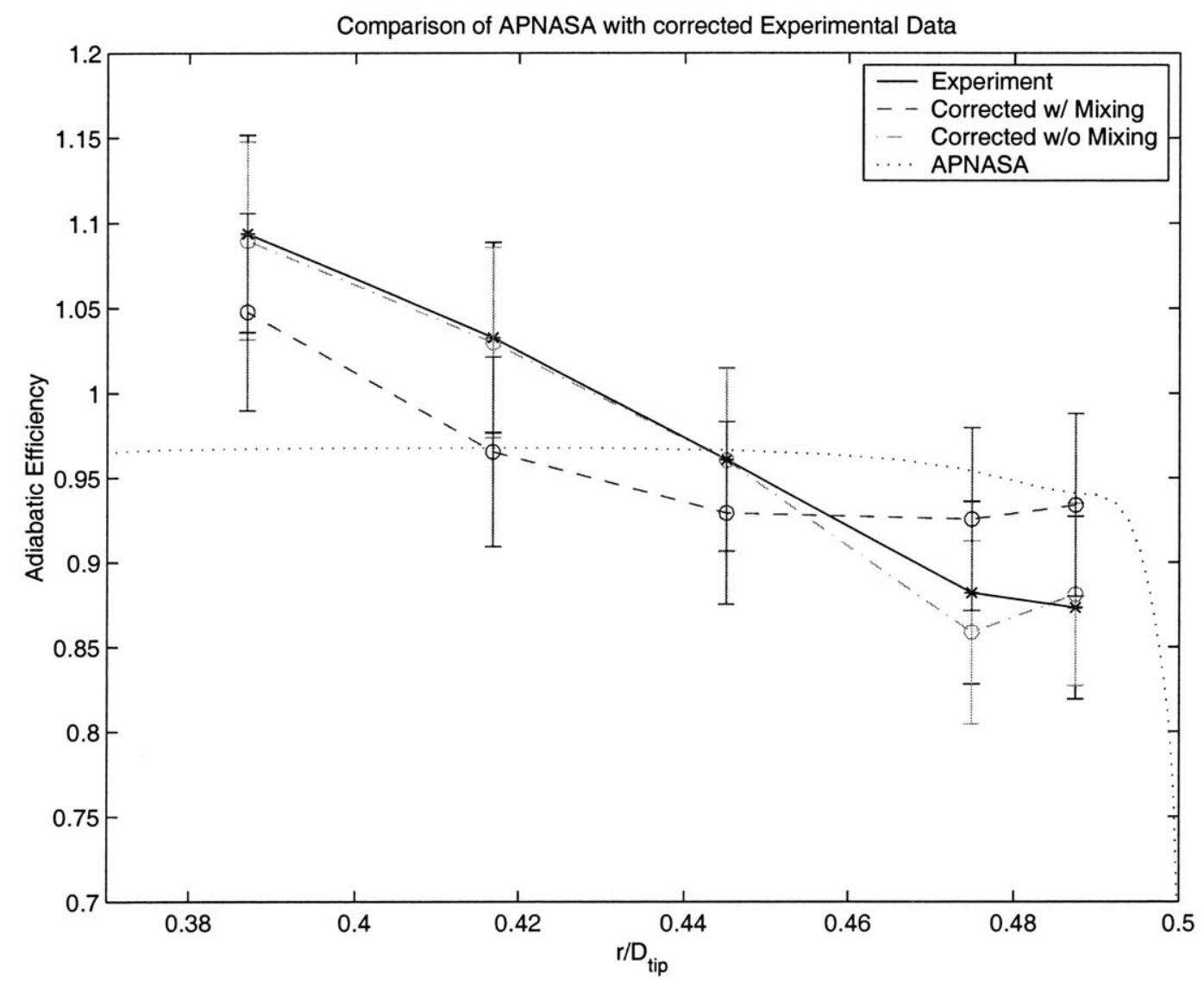

Figure 5-12: Effect of spanwise transport on adiabatic efficiency distribution

Given that the distance from the rotor trailing edge to the probe location is small, it 
is possible that the wake flows are not completely mixed out. Therefore the true solution lies between the efficiency profile corrected without mixing and the profile corrected with mixing. The overall results of this analysis are satisfactory considering that the vortex model is incompressible while the rotor cxit flowfield operates in the range of high subsonic Mach numbers so as to consider it compressible. The fact that the trend to bring the efficiency distribution closer to the APNASA values for both the hub streamtube and the tip streamtube is excellent, and the quantitative values predicted by the model are also satisfactory.

The measured efficiency is the true efficiency distribution of the stage. The APNASA efficiency distribution shown in figure 5-12 is the design intent efficiency distribution. From figure 5-12, it can be seen that the stage shows a higher than design efficiency in the hub region of the rotor. The tip region shows a lower than design efficiency. It is interesting to note though that the measured average efficiency of the rotor matches very well with the APNASA prediction. The vortex model indicates that one of the reasons for the discrepancy between the measured efficiency distribution and the APNASA distribution is spanwise transport within the blade wake due to unsteady vortex shedding. This radial tranport causes the spanwise redistibution of flow properties that accounts for at least part of the difference between the measured and predicted efficiency distributions. Therefore, in order to more accurately predict the rotor performance, a vortex model similar to the one used here should be added to existing fully $3-\mathrm{D}$, viscous computational tools. This addition could allow these analysis tools to more accurately predict rotor performance and more closely achieve design intent. 


\section{Chapter 6}

\section{Parametric Study}

\subsection{Introduction}

Aspiration is a new option that a compressor designer can use to create a new compression system. Therefore, a parameter study must be performed so that the designer has a rough idea where in the design space aspiration can be most effective. This chapter describes a parameter study for a single compressor stage that compares conventional designs with aspirated designs. The study includes compressibility effects, including shock losses, and a viscous loss model, and the parameters are calculated along the meanline of the stage with no radial variation.

\subsection{Model Development}

The primary benefit of an aspirated compressor is the increase in work level per stage. Therefore it is desirable to outline the benefits made available by aspiration and to determine the design space where these benefits can be exploited. To achieve this end, the compression system is characterized by a set of parameters, and the parameter space is limited so that realistic comparisons can be made between current compressor designs and aspirated designs.

The Leiblein diffusion factor [10] is widely accepted as a measure of the blade loading. Equations (6.1) and (6.2) define the diffusion factor for the rotor and stator with zero inlet 
swirl into the rotor and zero exit swirl from the stator, where $V_{1}^{\prime}$ is the rotor inlet relative velocity, $V_{2}^{\prime}$ is the rotor exit relative velocity, $v_{2}$ is the rotor exit tangential velocity, $V_{2}$ is the rotor exit absolute velocity, and $V_{3}$ is the stator exit velocity. For this parameter study the solidity for the rotor and stator is $\sigma_{R}$ and $\sigma_{S}$. The solidity is not varied in the results of the parameter study but does have a large influence on the performance of the stage.

$$
\begin{aligned}
& D_{R}=1-\frac{V_{2}^{\prime}}{V_{1}^{\prime}}+\frac{v_{2}}{2 \sigma_{R} V_{1}^{\prime}} \\
& D_{S}=1-\frac{V_{3}}{V_{2}}+\frac{v_{2}}{2 \sigma_{S} V_{2}}
\end{aligned}
$$

The geometry of the stage is characterized by the flow parameter $\phi=\frac{C_{x}}{U}$ where $C_{x}$ is the axial velocity and is held constant throughout the stage and $U$ is the blade speed. The blade geometry is characterized by the work parameter, $\psi=\frac{\Delta H}{U^{2}}$, where $\Delta H$ is the enthalpy rise provided by the rotor.

Now we can rewrite equations (6.1) and (6.2) in terms of $\psi$ and $\phi$ as shown by Kerrebrock [7] to get equations (6.3) and (6.4).

$$
\begin{gathered}
D_{R}=1-\left[\frac{\phi^{2}+(1-\psi)^{2}}{\phi^{2}+1}\right]^{\frac{1}{2}}+\frac{\psi}{2 \sigma_{R}}\left(\phi^{2}+1\right)^{-\frac{1}{2}} \\
D_{S}=1-\left(\frac{\phi^{2}}{\psi^{2}+\phi^{2}}\right)^{\frac{1}{2}}+\frac{\psi}{2 \sigma_{S}}\left(\psi^{2}+\phi^{2}\right)^{-\frac{1}{2}}
\end{gathered}
$$

\subsection{Compressibility and Losses}

The Mach number is noticeably absent from the above. For modern compressor stages compressibility, and more specifically shock strength and shock structure, play an important part in the design of high speed compressor blading. Therefore an extension of the previous 
formulation which includes compressibility will be presented. Shock losses and viscous losses will be estimated and used in the parameter study to predict the compressor design regime in which aspirated compressors would have the most benefit.

\subsubsection{Upstream of the Rotor}

The compressibility factor requires a another parameter in order to determine the flow Mach numbers within the stage. The rotor inlet axial Mach number was chosen as the extra parameter for one reason. The aspirated stages were designed as fan stages for either military or civil engines where a high mass flow per unit area is desired. This requirement sets the range of inlet Mach numbers to $M_{x}=0.6-0.7$.

After setting the inlet Mach number, the blade Mach number $\left(M_{b}\right)$ follows from the combination of inlet Mach number with the flow parameter $\phi$.

\subsubsection{Viscous Loss vs. Diffusion Factor Correlations}

Equation (6.6) defines the viscous loss correlation versus diffusion factor, where the viscous loss parameter, $\bar{\omega}$, is defined by equation (6.5). $P_{t}$ and $\beta$ are defined in the system relative to the blade, where subscript 1 represents the inlet condition, and subscript 2 is the exit condition. $\beta$ is the flow angle relative to the blade.

$$
\begin{gathered}
\bar{\omega}=\frac{P_{t 1}-\overline{P_{t 2}}}{P_{t 1}-P_{1}} \\
\bar{\omega} \frac{\cos \beta_{2}^{2}}{\cos \beta_{1}^{2}} \frac{\cos \beta_{2}}{2 \sigma}=A+B \frac{D_{f}}{F}+C e^{15(D-E)}
\end{gathered}
$$

For the standard design parameter study, the constants $A, B, C, E$, and $F$ are chosen to match the viscous loss data presented by Kerrebrock [6]. For the aspirated design parameter study, the constants $A, B$, and $C$ are assumed to remain the same as for a standard stage, while $F$ and $E$ are adjusted to allow for lower viscous losses at higher diffusion factor levels. 
Constant $F$ is increased from 0.45 to 0.65 , and constant $E$ is increased from 0.55 to 0.75 . The rationale for this is as follows.

The viscous loss of a blade row can be estimated using the trailing edge momentum thickness, and data has shown that the trailing edge momentum thickness varies with the diffusion factor of the stage. Therefore, if we know the diffusion factor of a modern compressor and the corresponding momentum thickness, then the constants $D$ and $E$ can be adjusted to match the aspirated designs in terms of trailing edge momentum thickness. The limit of low loss for modern compressors is a diffusion factor of about 0.45. The high speed aspirated compressor designed by Merchant has a diffusion factor of roughly 0.7. For a similar momentum thickness $\left(\frac{\theta}{c}\right)$ level giving similar viscous losses. Only one correlation is used to describe aspirated compressors. Figure (6-1) shows the loss correlations used for the standard design and aspirated design parameter study, and equations (6.8) and (6.9) show the values of the constants used for the correlations.

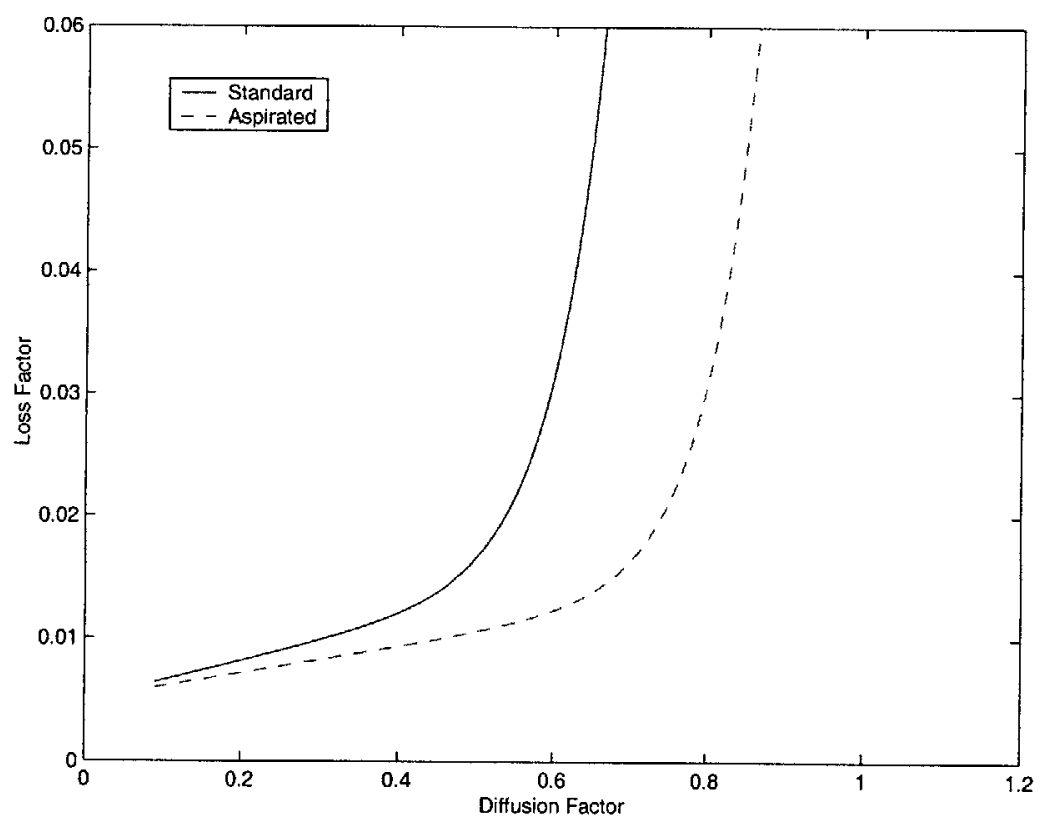

Figure 6-1: Loss correlation used for standard and aspirated stage design

$$
\begin{aligned}
& A=0.005 \\
& B=0.007
\end{aligned}
$$




$$
\begin{gathered}
C=0.008 \\
E=0.55 \\
F=0.45
\end{gathered}
$$

$$
\begin{gathered}
A=0.005 \\
B=0.007 \\
C=0.008 \\
E=0.75 \\
F=0.65
\end{gathered}
$$

To facilitate application to both rotating and stationary blades, the losses taken from figure (6-1) are converted to an entropy rise $(\Delta S)$ using equation $(6.10)$, where $M_{x}$ is the axial Mach number and $M_{b}$ is the blade relative tangential Mach number.

$$
\begin{array}{r}
\frac{\Delta S_{v i s c}}{R}=-\ln \left(1-\bar{\omega}\left(1-\frac{P_{1}}{P_{t 1}}\right)\right) \\
\frac{P_{1}}{P_{t 1}}=\left(1+\frac{\gamma-1}{2}\left(M_{x}^{2}+M_{b}^{2}\right)\right)^{-\frac{\gamma}{\gamma-1}}
\end{array}
$$

\subsubsection{Shock Losses}

For transonic compressor design, shock structure and losses can dominate the performance of a compressor stage. Therefore it is necessary to estimate the shock losses within the parameter study. Miller et al. [15] estimate the shock losses for a given blade row as the average of the loss produced by a normal shock at the inlet relative Mach number and the loss produced by a normal shock at the passage Mach number just ahead of the shock impingement location.

For the parameter study, the inlet relative Mach number can be calculated from the 
design conditions, but the shock impingement Mach number must be estimated. Using blade sections from the low speed and high speed fan stages designed by Merchant [14], equation (6.10) was used to predict the Mach number at the shock impingement location.

$$
M_{i m p}=1.225 * M_{r e l}
$$

Once the inlet Mach number and shock impingement Mach number are known, the normal shock equation (6.11) is used to determine the entropy rise generated across a shock at each of these Mach numbers, and these entropy rises are averaged to determine the shock losses for the blade row. The entropy rise due to viscous losses and shock losses are added to determine the total loss of the rotor.

$$
\Delta s=C_{p} \ln \left[\left(1+\frac{2 \gamma}{\gamma+1}\left(M_{1}^{2}-1\right)\right)\left(\frac{2+(\gamma-1) M_{1}^{2}}{(\gamma+1) M_{1}^{2}}\right)\right]-R \ln \left(1+\frac{2 \gamma}{\gamma+1}\left(M_{1}^{2}-1\right)\right)
$$

\subsubsection{Rotor-stator gap conditions}

The total temperature ratio $(\tau)$ produced by the rotor must be known in order to determine the stator inlet Mach numbers and is calculated using equation (6.12).

$$
\tau=1+\frac{(\gamma-1) \psi M_{b}^{2}}{1+\frac{\gamma-1}{2} M_{x}^{2}}
$$

With the axial velocity assumed to be constant throughout the stage, equation (6.13) gives the total Mach number into the stator.

$$
M_{2}^{2}=\frac{M_{x}^{2}+M_{b}^{2} \psi^{2}}{\tau\left(1+\frac{\gamma-1}{2} M_{x}^{2}\right)-\frac{\gamma-1}{2}\left(M_{x}^{2}+M_{b}^{2} \psi^{2}\right)}
$$

Finally, equation (6.14) gives the axial Mach number exiting the rotor $\left(M_{x 2}\right)$, and equa- 
tion (6.15) gives the blade Mach number going into the stator $\left(M_{b 2}\right)$.

$$
\begin{aligned}
& M_{x 2}=M_{x} \sqrt{\tau} \sqrt{\frac{1+\frac{\gamma-1}{2} M_{x}^{2}}{1+\frac{\gamma-1}{2} M_{2}^{2}}} \\
& M_{b 2}=M_{b} \sqrt{\tau} \sqrt{\frac{1+\frac{\gamma-1}{2} M_{x}^{2}}{1+\frac{\gamma-1}{2} M_{2}^{2}}}
\end{aligned}
$$

Now we have all the necessary parameters to define the performance of the stator in terms of viscous and shock losses. The viscous loss profile shown in figure 6-1 is also used to determine the viscous losses for the stator. Equation (6.16) is used to determine the diffusion factor of the stator given that the flow is turned back to axial.

$$
D_{s}=1-\frac{M_{x 2}}{M_{2}}+\frac{\psi M_{b 2}}{2 \sigma M_{2}}
$$

Once the viscous losses are known in the stator, then they are converted to an entropy increase defined by equation (6.18).

$$
\begin{gathered}
\frac{\Delta S}{R}=-\ln \left(1-\overline{\omega_{s}}\left(1-\frac{P_{2}}{P_{t 2}}\right)\right) \\
\frac{P_{2}}{P_{t 2}}=\left(1+\frac{\gamma-1}{1} M_{2}^{2}\right)^{-\frac{\gamma}{\gamma-1}}
\end{gathered}
$$

For the shock losses, the same process used for the rotor is used for the stator with $M_{2}$ as the relative inlet Mach number to the blade row. Therefore, viscous and shock losses from both the rotor and stator are presented as entropy rises which can be summed to give a total entropy rise for the stage. Using this entropy rise and $\tau$, equation (6.18) produces the total pressure rise $(\pi)$ for the stage, and equation (6.19) gives the adiabatic efficency $(\eta)$ of the stage. 


$$
\begin{gathered}
\pi=\frac{\tau^{\frac{\gamma}{\gamma-1}}}{e^{\frac{\Delta s}{R}}} \\
\eta=\frac{\pi^{\frac{\gamma-1}{\gamma}}-1}{\tau-1}
\end{gathered}
$$

\subsection{Results of the Parameter Study}

The application of boundary layer control to increase blade loading opens up the compressor design space and allows the compressor designer to achieve designs not previously possible due to constraints imposed by boundary layer separation. Aspiration allows an increase in the practical range of compressor diffusion factors thus allowing higher blade loadings. Higher blade loadings can enable: higher pressure ratio per stage for a given blade speed and lower blade speed for given pressure ratio.

This section outlines the results of the parameter study shown in the previous section. Previous CFD results have shown that aspiration increases the throughflow efficiency of a

given design by reducing the trailing edge momentum thickness. This parameter study has investigated the design space of current conventional designs as well as aspirated designs in order to determine where aspiration would have the most beneficial effect. For this parameter study, a matrix of solutions was generated over the three-dimensional parameter space defined by $0.5<\phi<1.1,0.2<\psi<1.2$, and $.45<M_{x}<.75$.

\subsubsection{Parametric Comparison of Conventional and Aspirated De- signs}

In this section, the meanline parameter study is compared with some existing aspirated and conventional compressor designs in order to assess the accuracy of the model. Two existing aspirated designs are compared to the model. These fan designs were performed by Merchant, and the detailed designs can be found in reference [14]. One is the low speed aspirated design 
that is the focus of this thesis. The other aspirated design is a high speed design that produces a pressure ratio of 3.5 . One conventional design that is used to compare is the first stage of a low aspect ratio two-stage fan designed at NASA. The details of this experiment are described in reference [25]. The other conventional stage is the high throughflow, low hub/tip ratio compressor design of Wennerstrom. The details of this compressor's performance can be found in reference [28].

Figure 6-2 compares the stage pressure ratio and efficiencies of the two aspirated designs and the two conventional designs. The low speed aspsirated stage has a meanline $\phi$ of 1.1 , a $\sigma$ of 1.8 , and an $M_{1}$ of 0.64 . The high speed aspirated design has $\phi=.62, \sigma=2.7$, and $M_{1}=0.65$. The NASA first stage has $\phi=.57, \sigma=2$, and $M_{1}=0.58$. Finally, the Wennerstrom stage has nearly the same meanline parameters as the NASA stage with $\phi=.575, \sigma=2.2$, and $M_{1}=0.58$. Therefore, since the parameter study results for the Wennerstrom stage and the NASA stage would have been very close to each other, only one line is plotted for those two stages.

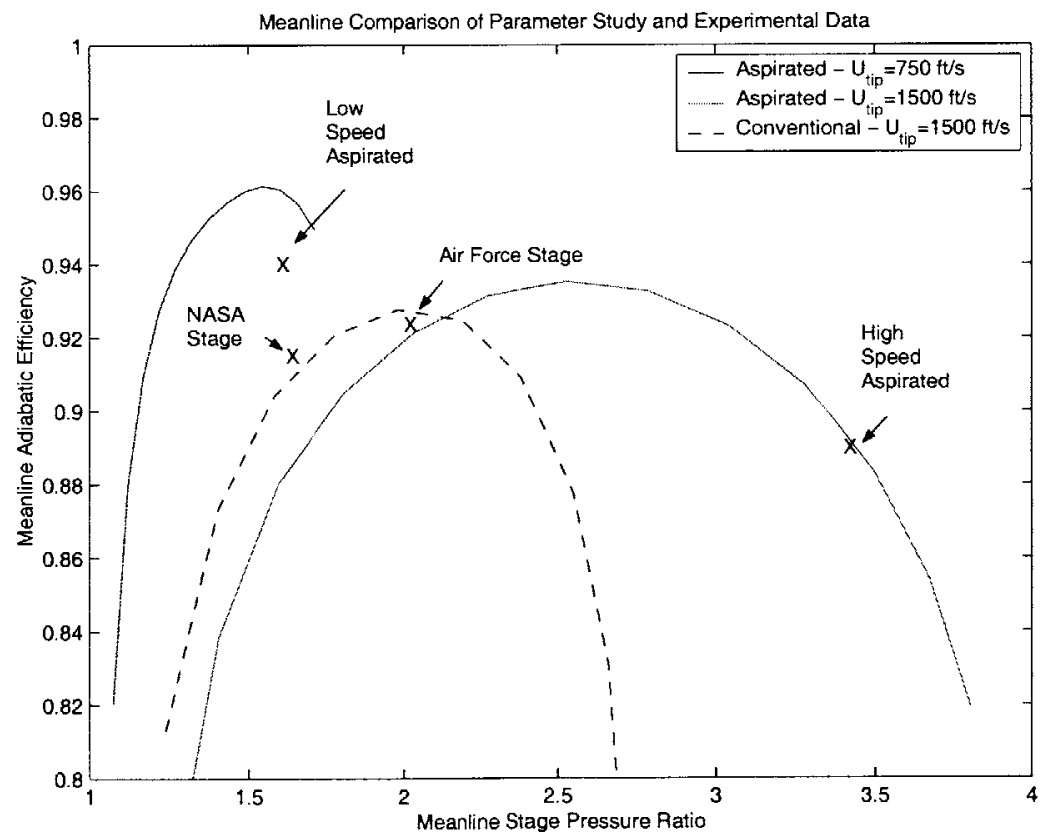

Figure 6-2: Comparison of Meanline Parameter Study to Compressor Data - Stage Efficiency

The parameter study overestimates the meanline efficiency of the low speed aspirated stage by about 1.5 percentage points. A redesigned stator could account for part of the discrepancy between the parameter study and the experimental data. The parameter study 
does a good job of estimating the meanline efficiencies of the other stages. Looking at some of the details of the parameter study and compressor stages, we can see from figure 6-3 that the parameter study does predict the stator inlet Mach number at the mean streamline very well. In order to get a better view of the whole compressor, it would be useful to extend the parameter study to radial variations through the stage. The extended parameter study is discussed in appendix D.

The conventional stages presented here are designs that are more than 20 years old. They were chosen because of access to detailed flow measurement data for comparison with the parameter study. Modern fan stages produce pressure ratios of 1.6 for adiabatic efficiencies of $91-92 \%$ adiabatic efficiency with meanline efficiencies probably on the order of $93-94 \%$ adiabatic efficiency.[21] The aspirated fan stage with a redesigned stator should produce a meanline adiabatic efficiency slightly higher than the $94 \%$ from the experiment. This corresponds well with the predictions of the parameter study shown in figure 6-4. These plots show that at low pressure ratios around 1.6, the aspirated designs should have 1-1.5\% increase in meanline adiabatic efficiency over conventional designs.

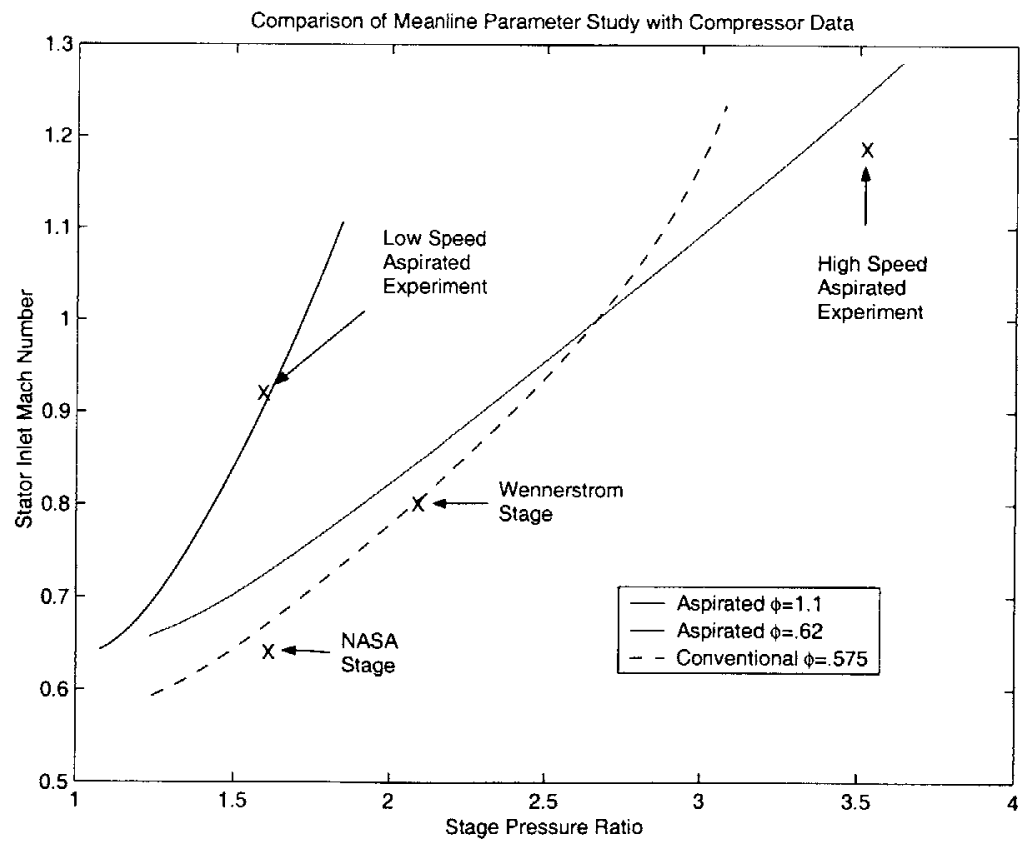

Figure 6-3: Comparison of Meanline Parameter Study to Compressor Data - Stator Inlet Mach Number 


\subsubsection{Constant Mass Flow}

In order to simplify the investigation, this section focuses on comparisons for a given inlet axial Mach number of 0.65 . For fan stages, it is important to have a large mass flow per unit area which is directly related to the inlet axial Mach number. The aspirated fan stages designed by Merchant have inlet axial Mach numbers of 0.65 . Other commercial and military fan stages such as the F119 fan stage have similar inlet Mach numbers. Therefore, $M_{x}=0.65$ is chosen for this comparison. A later section will describe the effects of inlet axial Mach number. The solidity $(\sigma)$ of each stage is set to 2.0 .

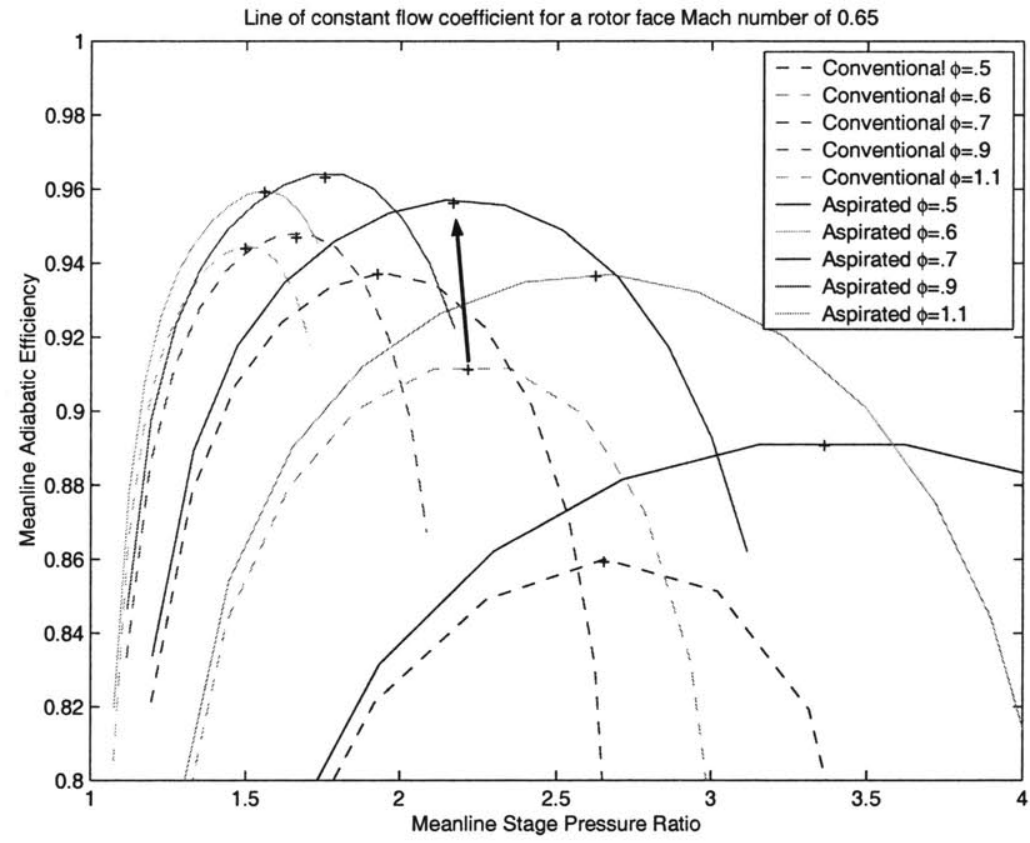

Figure 6-4: Stage performance over the given parameter range for $M_{x}=0.65$

Figure 6-4 shows the stage pressure ratio versus stage efficiency for several mass flow parameters $(\phi)$ and a range of blade loadings $(\psi)$ for an inlet axial Mach number of 0.65 . The first important thing to note is that the aspirated loss correlation always results in an increase of the efficiency of the stage and also an increase of the pressure ratio corresponding to the peak efficiency point for a given $\phi$. Therefore, compared to a conventional compressor stage designed to a given pressure ratio and mass flow, an aspirated design tends to a higher $\phi$ (or lower blade speed) in order to achieve maximum efficiency.

It is important to point out the limitations of this study. The efficiencies presented are 
for the main flow only and do not take into account the flow removed from the flowpath. Therefore, in an engine application, the suction flow would have to be accounted for and may count against the overall efficiency of the stage. This parameter study also does not account for any shock-boundary layer interaction. The viscous losses and the shock losses are calculated separately, and may give unrealistic design possibilities.

With these limitations in mind, some direct comparisons can be made between conventional designs and aspirated designs. Figure 6-4 shows a conventionally designed compressor that can produce a meanline pressure ratio of 2.2 at an efficiency of $91 \%$ and a flow coefficient of 0.6. If aspiration is applied to this design to help the boundary layer, then the efficiency increases by $1.5 \%$ for the same flow coefficient. But if a fully aspirated design is considered to achieve a pressure ratio of 2.2 , the optimum flow coefficient increases to 0.7 , and the meanline efficiency has increased to about $95 \%$. This change is shown by the arrow. For a given meanline pressure ratio, an aspirated design wants to decrease the blade speed of the stage and achieve the pressure ratio through turning the flow.

\subsubsection{Maximum Efficiency Designs}

This section presents results of the parameter study that deal only with the peak efficiency points for designs at a given flow parameter and inlet Mach number. For compressor design, mass flow and pressure ratio are specified, and then the blading is designed to the maximum efficiency points. The plots in this section show the effect of aspiration on several parameters for these maximum efficiency points with inlet axial Mach number as a parameter.

Figure 6-5 shows only the maximum efficiency points for each flow coefficient for a range of inlet axial Mach numbers. Flow coefficient decreases as the pressure ratio increases along the constant inlet axial Mach number lines. For a given pressure ratio, this figure shows that the efficiency gain going from a standard design to an aspirated design increases as the pressure ratio gets larger. The high speed and low speed aspirated designs are shown in figure 6-5.

Figures 6-6 and 6-7 show the diffusion factors for the rotor and stator corresponding to the maximum efficiency points shown in figure 6-5. The aspirated designs show a much 


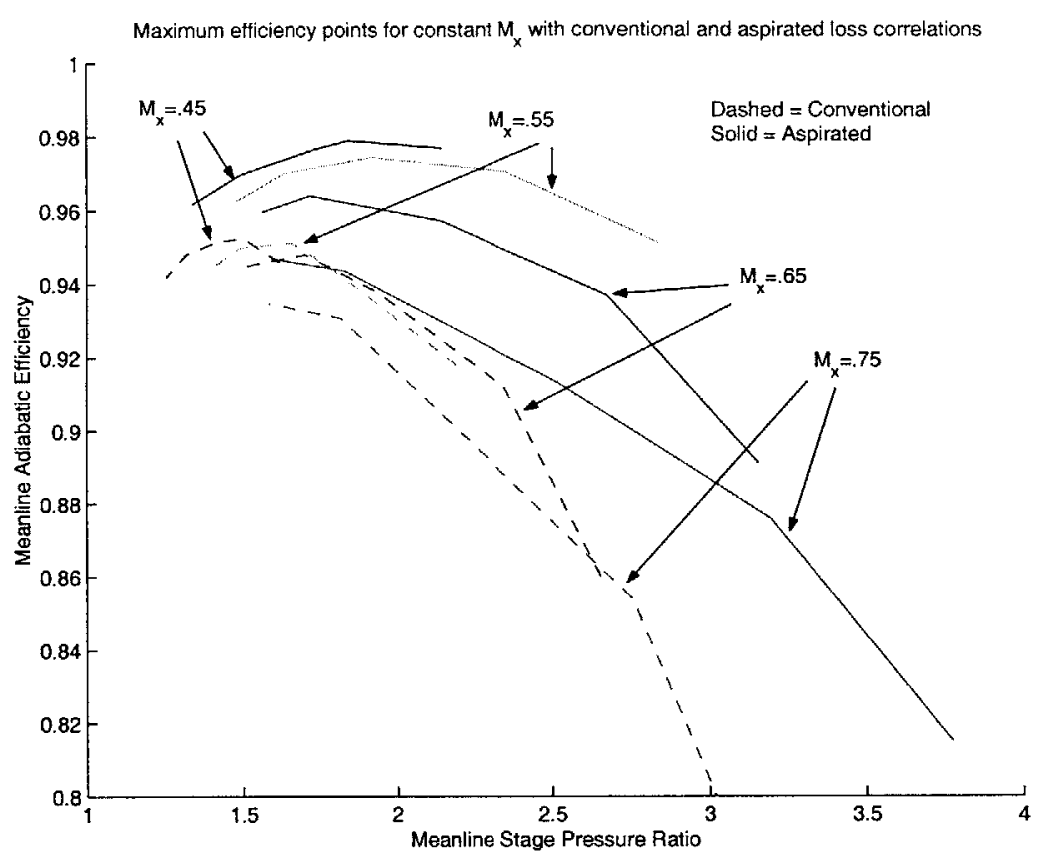

Figure 6-5: Maximum efficiency points for a range of inlet axial Mach numbers

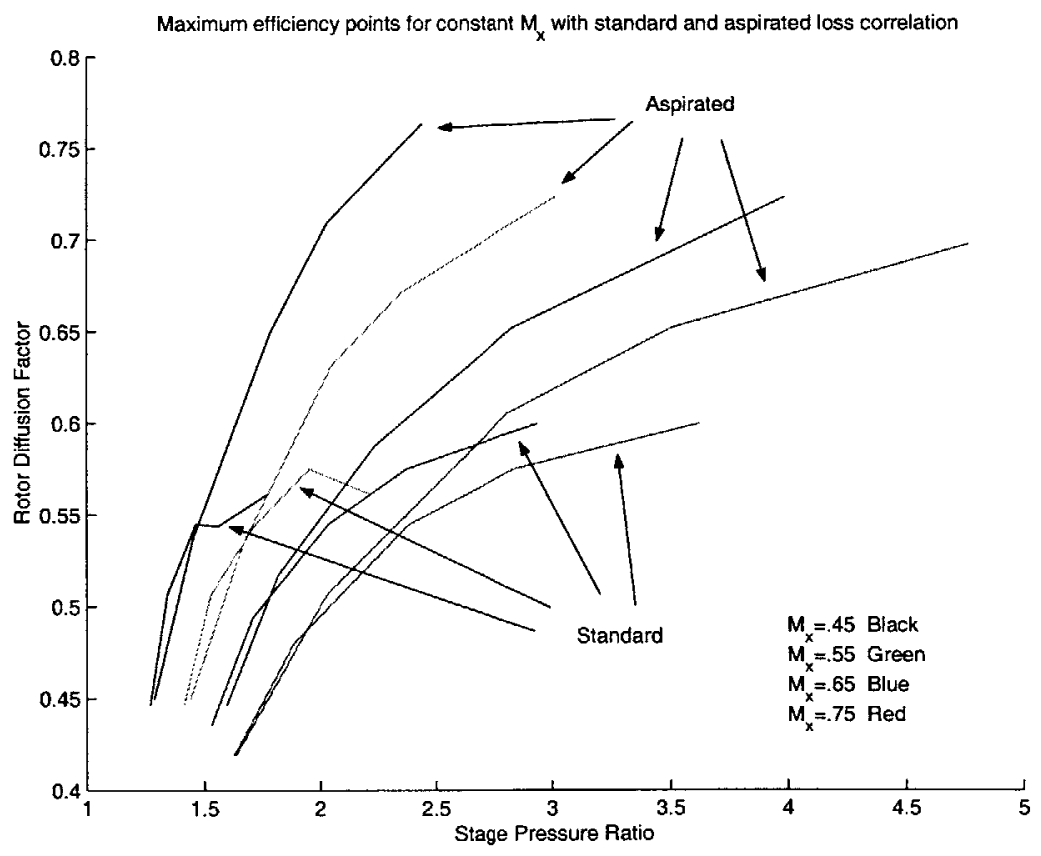

Figure 6-6: Rotor diffusion factor for the maximum efficiency points 


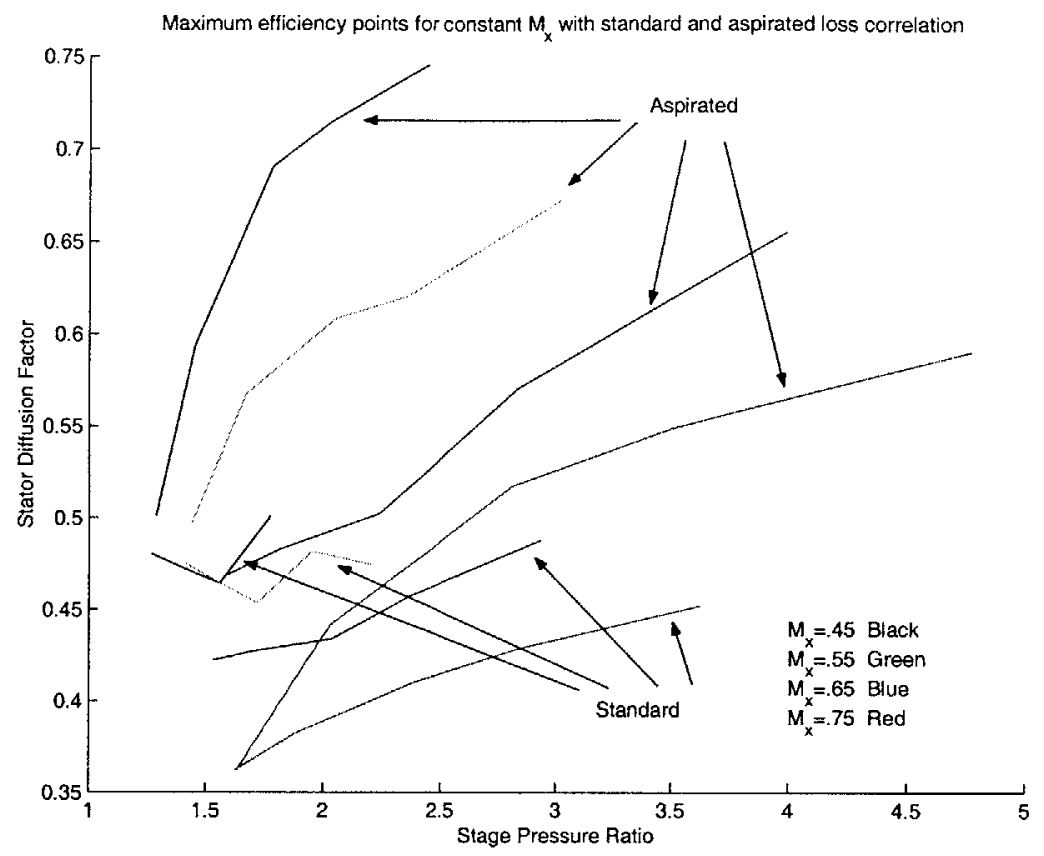

Figure 6-7: Stator diffusion factor for the maximum efficiency points

higher diffusion factor for both the rotor and stator than the conventional designs. The low speed aspirated design has a rotor and stator diffusion factor of 0.5 , while the high speed aspirated design has a rotor and stator diffusion factor near 0.7 .

Figure 6-8 shows the stator inlet Mach number for the maximum efficiency points. For all pressure ratios for given rotor inlet Mach number, the stator inlet Mach number is higher for the aspirated design than for the conventional design. The larger velocity into the stator is due to the larger turning provided by the aspirated design. In order to achieve the pressure ratio, the standard designs rely on blade speed while the aspirated designs rely on turning the flow more.

Finally, figure 6-9 shows the work coefficients for the maximum efficiency points. Again, the aspirated designs allow for higher work than the standard designs. The work is increased because the aspiration allows for much higher turning angles. 


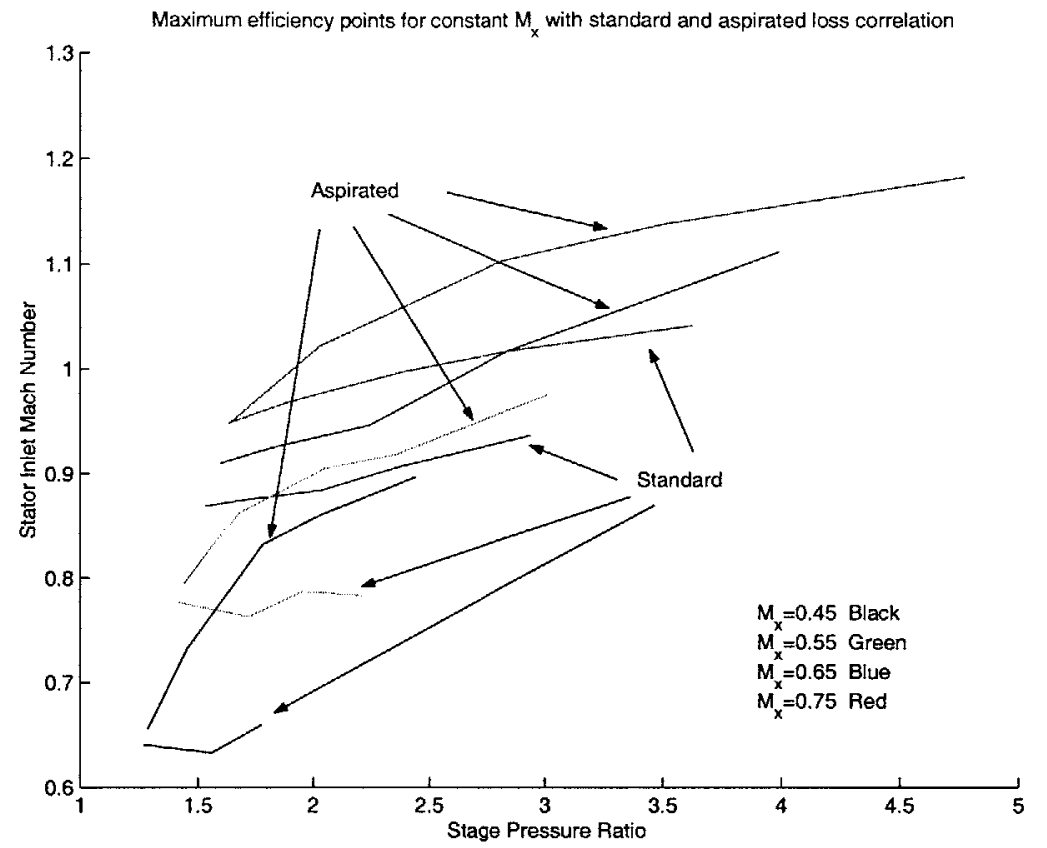

Figure 6-8: Stator inlet Mach number for the maximum efficiency points

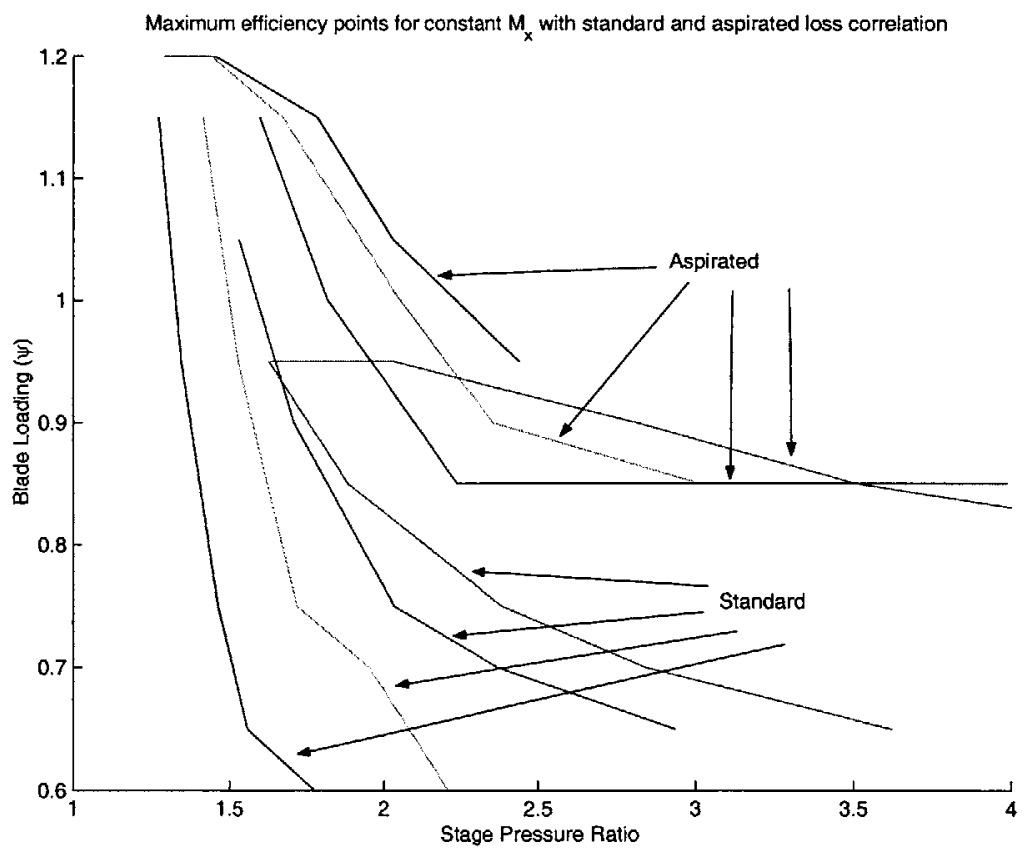

Figure 6-9: Blade loading $(\psi)$ for the maximum efficiency points 


\subsection{Meanline Parameter Study Limitations}

The main limitation of the meanline parameter study is the one-dimensionality. No special consideration is given to the parts of the blades that limit the performance. This limitation leads to predictions of overall stage pressure ratio and efficiency for conventional designs that are higher than have experimentally been shown. Predictions for aspirated designs are also affected by this limitation. The solution to this limitation is to extend the parameter study to account for radial variation of the streamlines through the stage. By allowing for radial variation through the stage, the parameter study can compare the performances of hub, midspan, and tip streamlines. By studying the individual streamlines, the weaknesses of each design can be investigated.

For high speed stages the shock-boundary layer interaction has a huge impact on the losses of the stage, and the parameter study does not take this source of losses into account. Therefore, the study overpredicts the pressure ratio and efficiency that high speed conventionally-designed compressor stages can achieve. Appendix D shows how the parameter study can been modified to include these effects, and compares the results with aspirated and conventional designs.

\subsection{Summary}

This parameter study presents a simple view of the compressor design space for both conventional and aspirated stage designs. Beginning with a simple meanline study, aspirated designs are compared to conventional designs. The model's equations are derived for a constant axial velocity and constant radius through the stage. Viscous and shock losses for both the rotor and stator are calculated and used to determine the stage efficiency. The Leiblein diffusion factor is used to correlate the viscous losses for each blade row. For conventional designs the viscous losses increase rapidly around a diffusion factor of 0.55 while for aspirated designs the viscous losses increase quickly near a diffusion factor of 0.75 . The removal of a fraction of the blade boundary layer allows an aspirated blade to do more diffusion of the flow compared to a conventional blade and still achieve the same trailing edge momentum 
deficit.

This meanline parameter study compares favorably to both aspirated and conventional compressor designs when studying the trends and approximate magnitudes of various parameters important to compressor design. One particular shortcoming of the meanline parameter study is the consistent overprediction of the stage efficiency. The neglecting of the losses in the hub and tip streamlines leads to this overprediction. Another shortcoming of the meanline study is the neglect of the effect of shock strength on the boundary layer thickness. To address these issues, the meanline parameter study is extended to include radial variations through the stage so that parameters on individual streamlines could be calculated. Also, the losses that are associated with the effect of shock strength on the boundary layer are estimated to first order using the velocity ratio across the passage. This approach gives a better estimate of the losses for blade sections with high inlet Mach numbers. 


\section{Chapter 7}

\section{Stator Redesign}

\subsection{Introduction}

Producing a fan stage that exhibits the high loading levels associated with aspirated fan designs without the penalty on overall engine efficiency is a difficult task. The goal of this thesis is to produce an aspirated fan stage that produces a pressure ratio of 1.6 at a throughflow adiabatic efficiency of $92 \%$. The fan stage tested in the Blowdown Compressor facility produced only a throughflow efficiency of $90 \%$. The APNASA analysis and experimental data show two low total pressure regions at the stage exit that contribute heavily to the stator losses.

This chapter presents the redesign of the aspirated fan stator, performed initially by Merchant [13], with a zero exit swirl stator and then expanded to include a stator with non-zero exit swirl.

\subsection{Redesigned Stator without Swirl}

Two problems are addressed in the redesign of the stator. First, reduction of the low total pressure regions located on the endwalls at the stator exit is achieved through a better blade design . Second, there is a small mismatch in the hub flowpath slope at the matching point between the rotor and the stator. This mismatch is smoothed out in the new stator design. 
The new stator hub flowpath had to be lowered to meet the matching requirement. The lower hub means that the stator exit Mach number is lower compared to the orginal design. A lower exit Mach number means that the stator must diffuse the flow more. As a result the stator loading increases. So the challenge is to design a more efficient stator with higher loadings.

Figures 7-1 and 7-2 show the blade surface Mach number and blade to blade Mach number contour of the hub section of the redesigned stator. The inlet Mach number is supersonic, and the passage shock is fairly strong. The shock loss is approximately two thirds of the total loss. The flow undergoes a slight precompression from a peak Mach number of 1.4 to a Mach number of 1.3 just ahead of the passage shock. The exit Mach number of the blade section is 0.5 compared to 0.72 for the original stator hub section. The pressure surface undergoes an adverse pressure gradient over $70 \%$ of the blade chord. The diffusion factor is 0.67 , compared to 0.54 for the original stator.

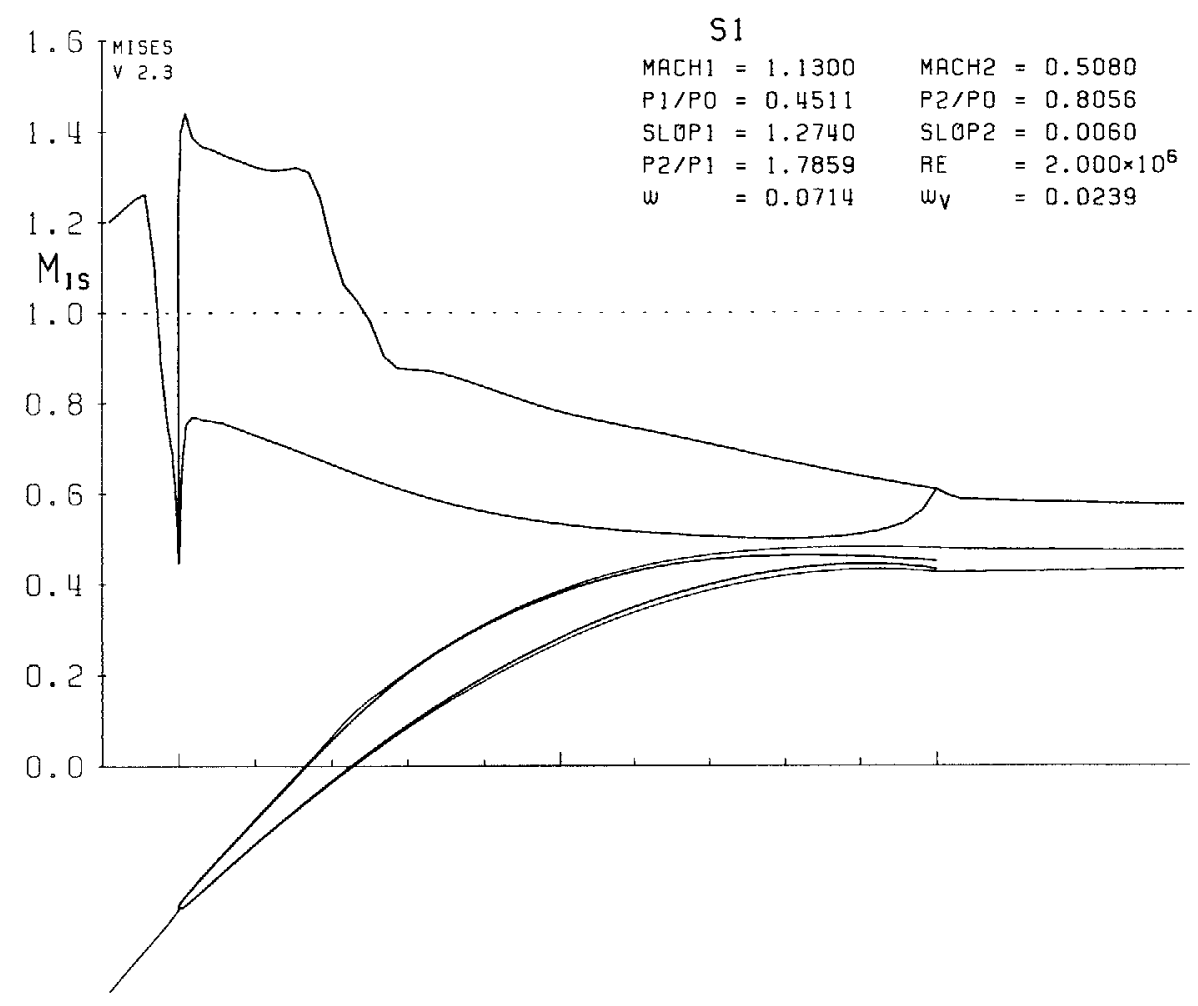

Figure 7-1: Blade surface Mach number distribution for the hub section without exit swirl

Figure 7-3 presents the boundary layer growth on the suction surface. The dashed line is $\delta^{*}-\dot{m}_{\text {suct }} / \rho_{e} u_{e}$ (removed streamtube height). The suction is applied at $25 \%$ chord. Figure 


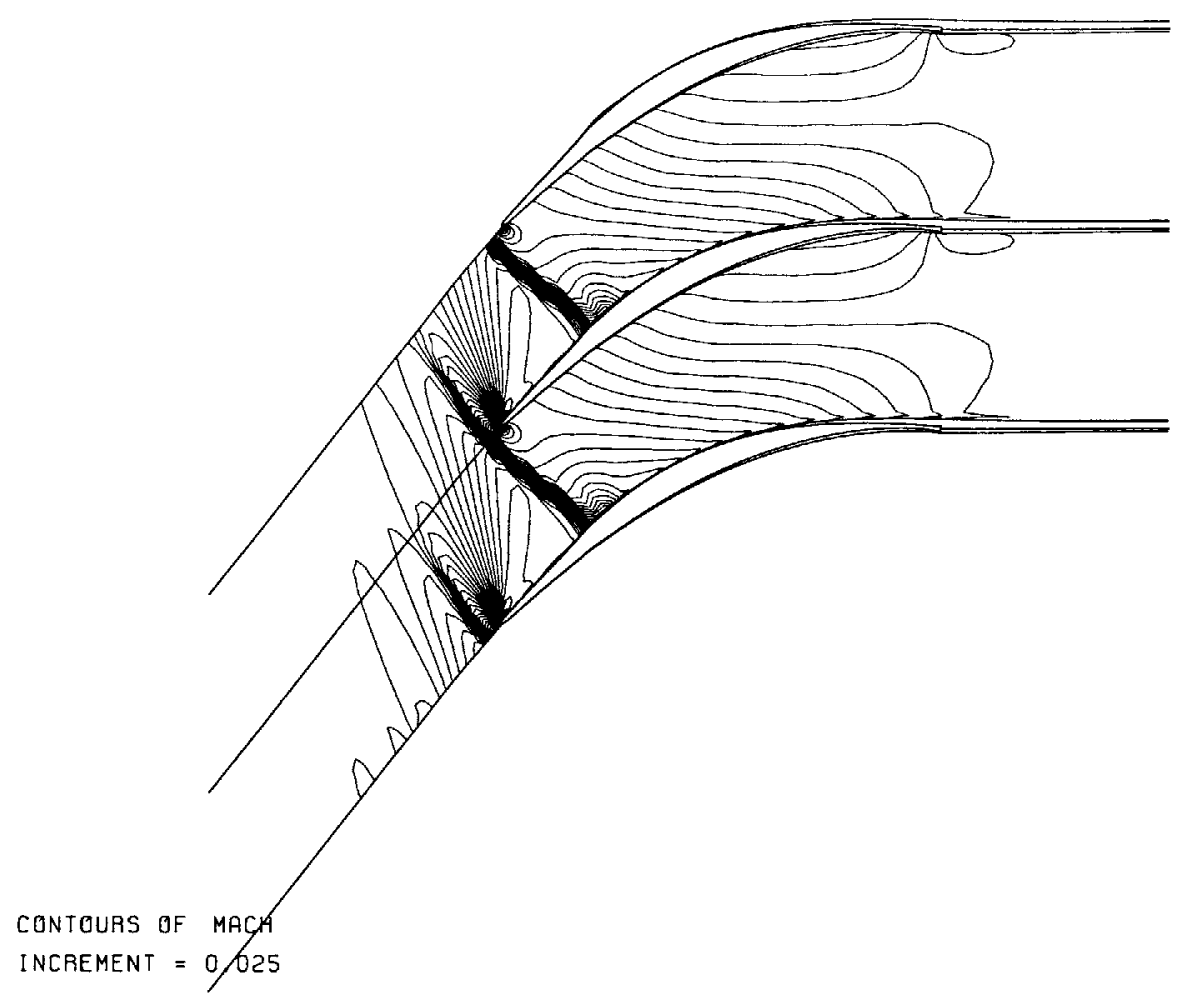

Figure 7-2: Mach number contour for the hub section without exit swirl

7-4 shows the effect suction has on the boundary layer as the shape parameter, $H_{k}$ decreases at $25 \%$ chord. 


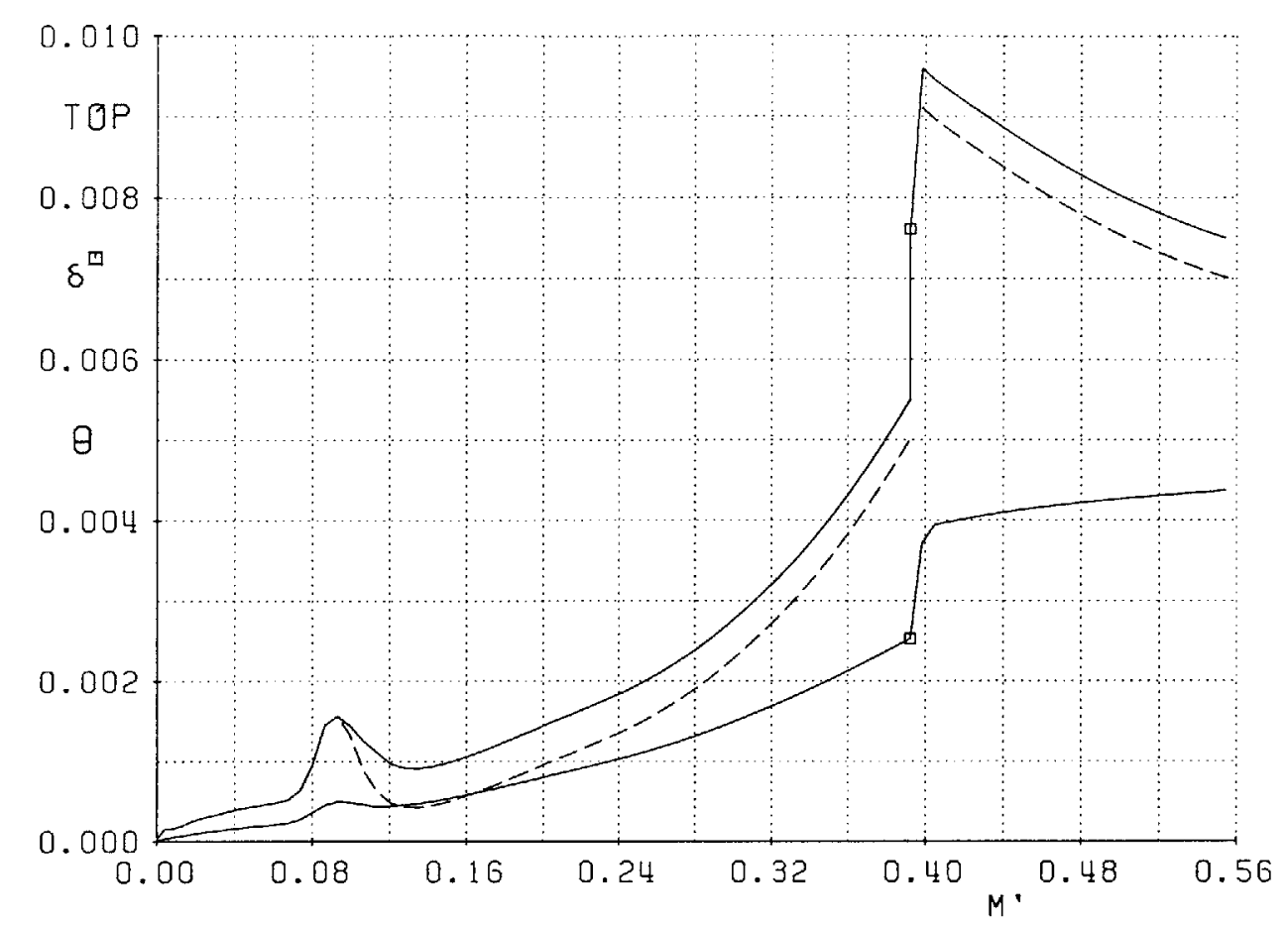

Figure 7-3: Suction surface boundary layer growth for the hub section without exit swirl

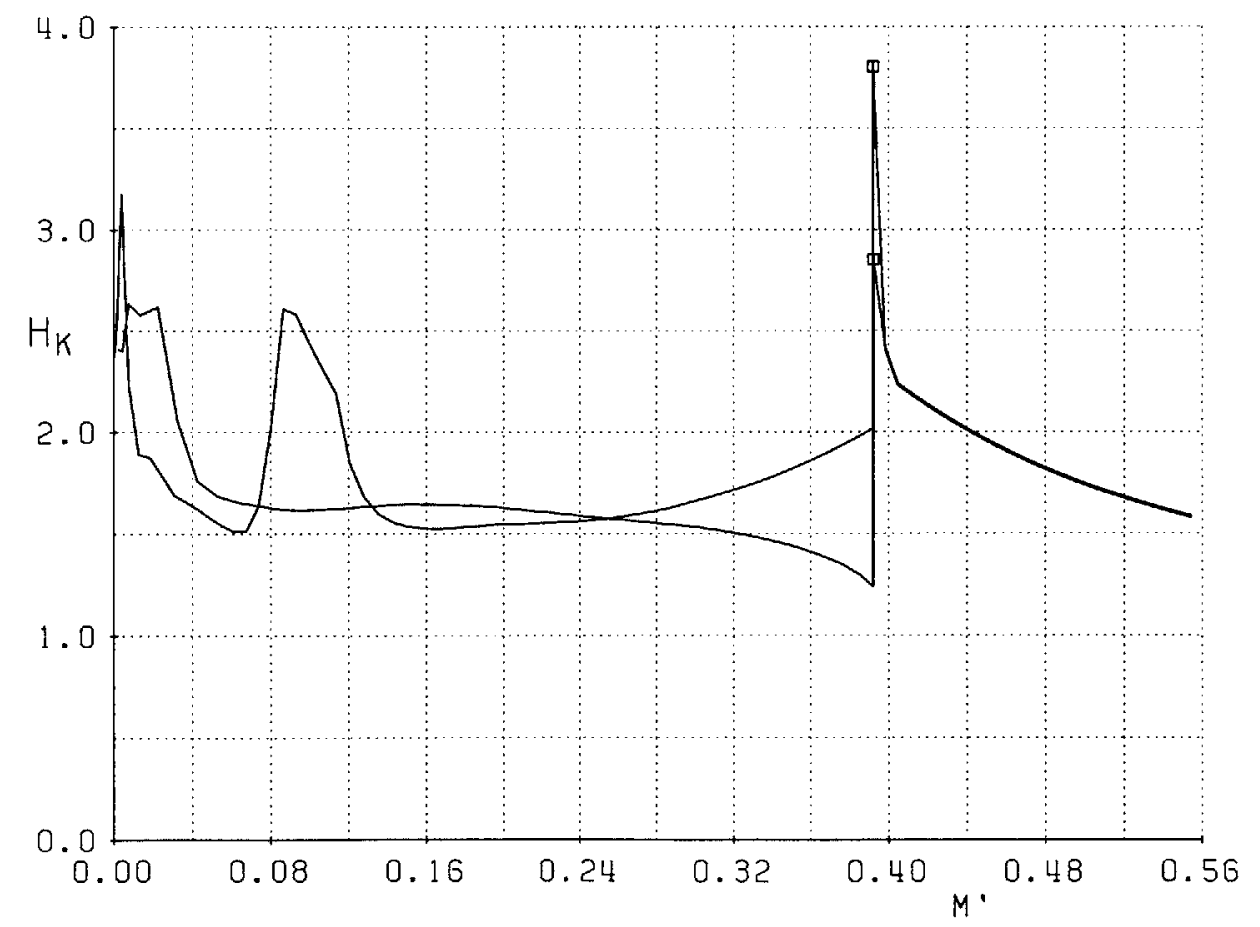

Figure 7-4: Boundary layer shape factor for the hub section without exit swirl 


\subsection{Redesigned Stator with swirl}

In order to lessen the loading of the stator hub, a new stator was designed with residual swirl left in the lower third of the stator. By unloading the stator hub sections, the viscous losses could be lowered, and the losses created by the interaction with the hub endwall boundary layer could possibly be lessened. Figure 7-5 presents the blade surface Mach number distribution for the hub section with exit swirl. The inlet Mach number is 1.13. The distribution shows a peak Mach number of near 1.4 with some slight precompression down to a Mach number of 1.3 just before the passage shock. The aspiration is applied just downstream of the passage shock impingement location.

Figure 7-5 shows the Mach number distribution on the blade surface. Similar to the section with no swirl, the peak Mach number is near 1.4. The precompression lowers the Mach number into the passage shock. The diffusion factor for this section has been lowered to 0.62 by leaving 14 degrees of swirl in the exit flow. Figure $7-6$ shows the blade to blade Mach number contour.

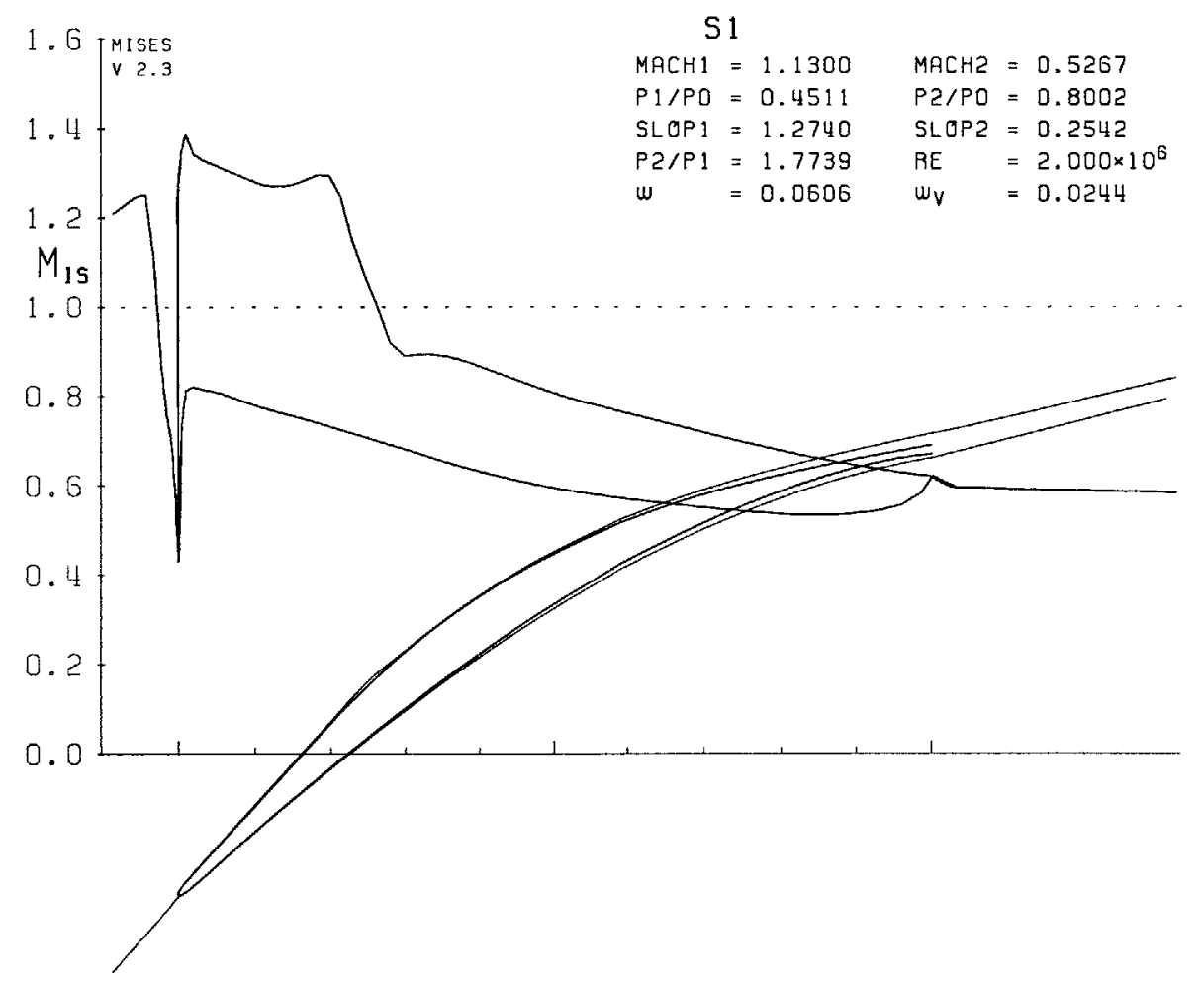

Figure 7-5: Blade surface Mach number distribution for the hub section with exit swirl 


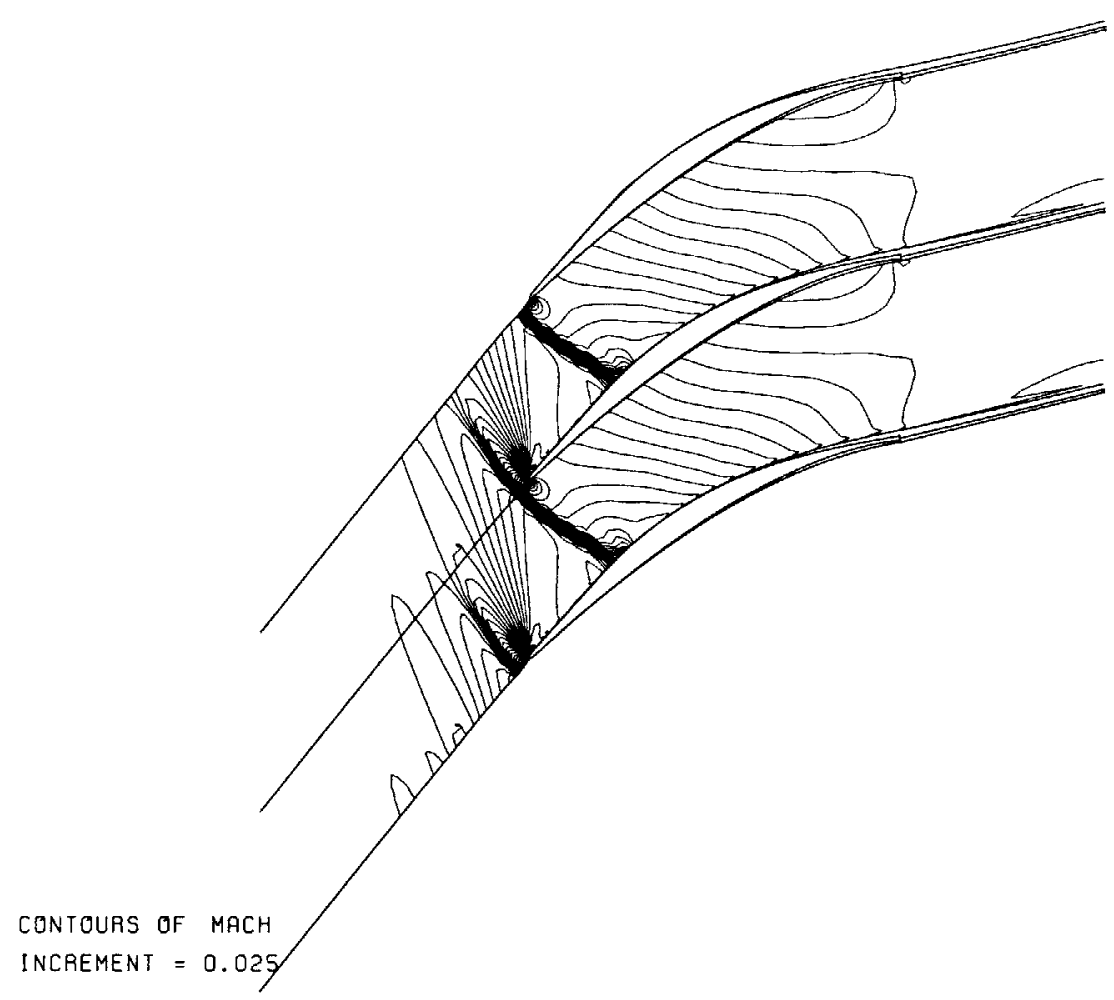

Figure 7-6: Mach number contour for the hub section with exit swirl

Figure 7-7 presents the boundary layer growth on the suction surface. The suction is applied at $25 \%$ chord again. The suction surface momentum thickness and displacement thickness at the trailing edge is approximately $15 \%$ smaller on the 'with swirl' design than the 'without swirl' design. Figure 7-8 clearly shows the effect of suction on the boundary layer with the reduction in shape parameter around $25 \%$ chord.

This design process was performed on the stator sections up to $30 \%$ span. The hub section was allowed the most exit swirl. The amount of exit swirl then decreased linearly back to zero at the $30 \%$ span blade section. This design scheme has the benefit of unloading the most highly loaded section of the stator. By keeping the upper $70 \%$ of the stator with no exit swirl flow condition, the goal of using this stage as a civil fan stage is still a possibility. 


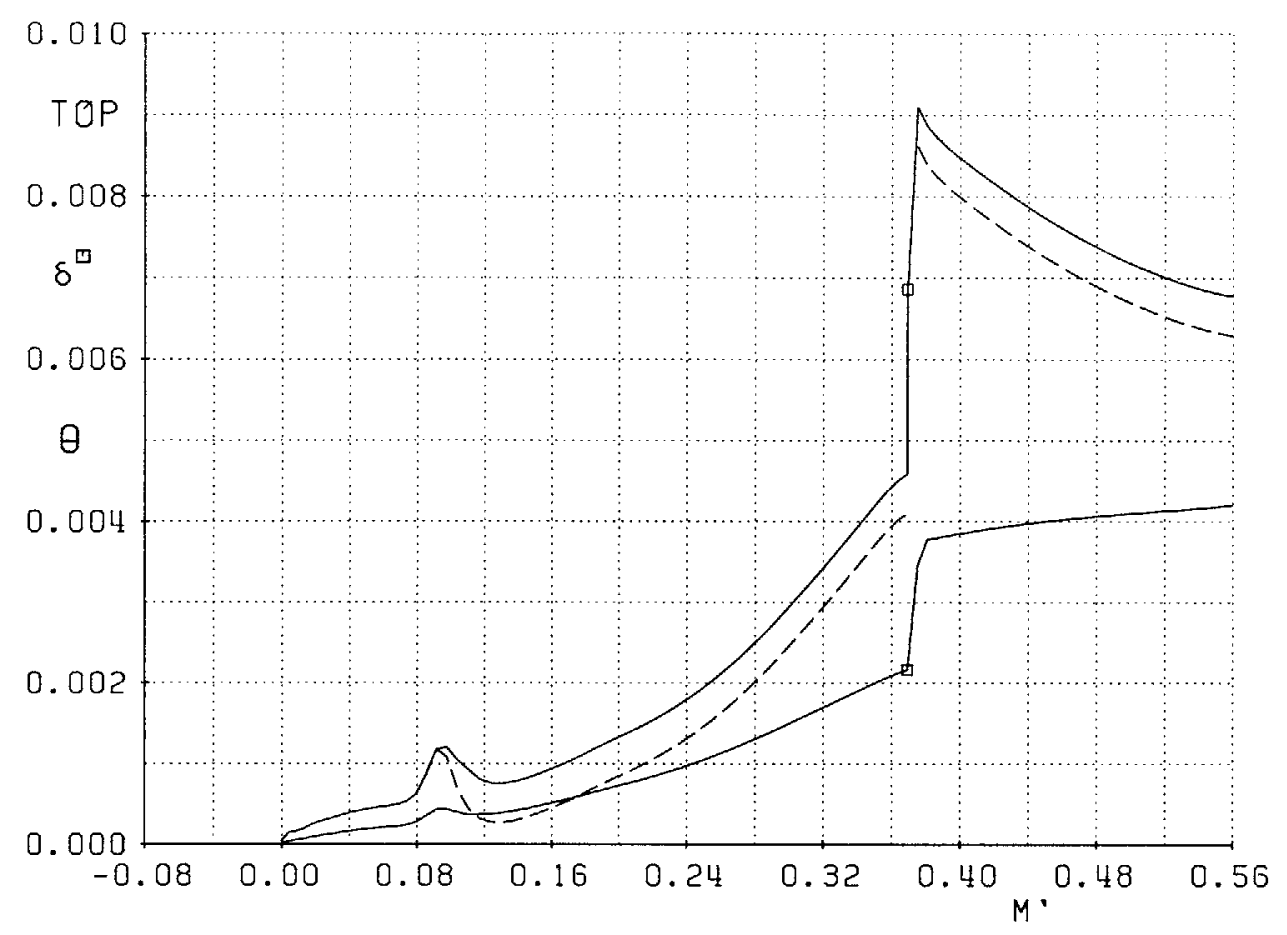

Figure 7-7: Suction surface boundary layer growth for thephub section with exit swirl

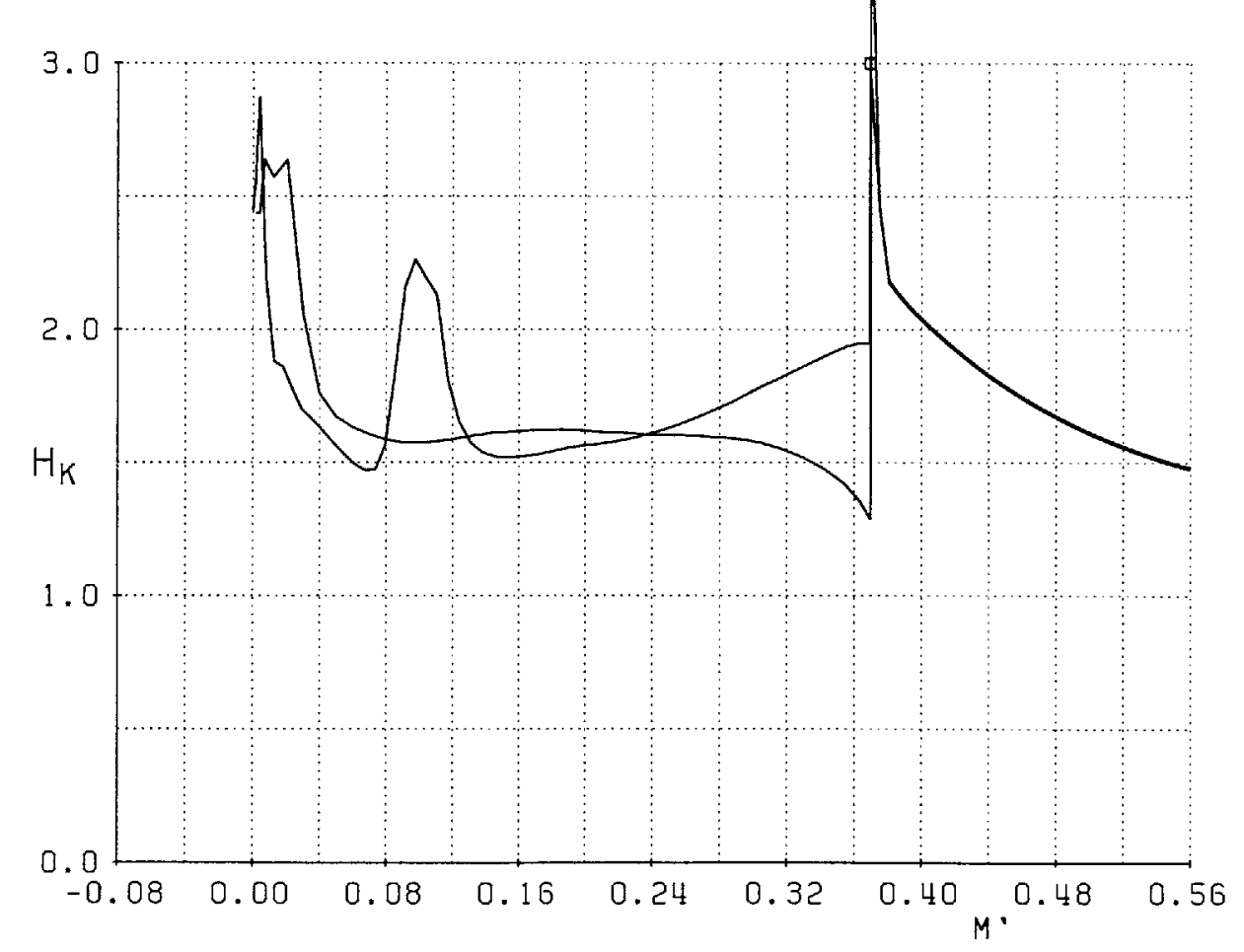

Figure 7-8: Boundary layer shape factor distribution for the hub section with exit swirl 


\subsection{Results}

Merchant [13] has produced a new stator that gives a stage throughflow efficiency of $92 \%$. This stator expands the flow to a lower Mach number than the original stator and turns the flow back to axial. A modification of this design has been made to decrease the loading near the hub in order to gain yet further benefit in the stage efficiency. By allowing the stator to leave swirl in the flow at the exit, the diffusion factor of the hub section has been decreased from 0.67 to 0.62 . The lower loading results in a smaller suction surface boundary layer at the trailing edge. While MISES is very useful as a design tool and allows very detailed control over the blade shape, the final design must be analyzed in a fully $3-\mathrm{D}$, viscous analysis. 


\section{Chapter 8}

\section{Conclusions, Contributions, and Future Work}

\subsection{Contributions}

This chapter describes the contributions made and the conclusions achieved through this research as well as describing the future work that could be done to better understand aspiration and its effects on axial compressor design.

The most significant contribution of this thesis is the presentation of the first experimental data of a fully aspirated fan stage. Detailed design point data compare very well with an APNASA 3D, viscous analysis, especially if account is taken of the effects of flow unsteadiness not included in the analysis. The rotor outflow measurements of total pressure ratio and rotor exit flow angle show good agreement with the APNASA analysis. The stator total pressure contours also agree well with the predictions of the APNASA analysis. These results validate the suction model used in the design and analysis tools. The off-design performance of the stage on the $100 \%$ design speedline also shows very significant results. APNASA could not predict a solution lower than $61 \frac{\mathrm{lbs}}{\mathrm{s}}$ mass flow while the experimental data shows unstalled stage performance at least down to $50 \frac{\mathrm{lbs}}{\mathrm{s}}$ mass flow with the stage performance following the trend predicted by both the APNASA analysis and a Pratt \& Whitney 3D, viscous analysis.

The use of an unsteady vortex shedding model has provided insight into the redistribution 
of flow quantities through radial transport induced by the vortex shedding. By adjusting the measured spanwise efficiency distribution with the results of the model, the data more closely matches the predicted efficiency distributions of APNASA.

A preliminary parametric study is one contribution of this thesis that is directed towards the better understanding of how aspiration affects compressors. A meanline study investigates a wide area of the compressor design space to determine under what flow conditions and design constraints aspiration could have the largest benefit on compressor performance. A few conventional and aspirated compressor designs are compared against the meanline study. The meanline study is then extended to include radial variations through the compressor stage to allow comparisons of hub, midspan, and tip streamlines for different conventional and aspirated designs.

One final contribution of this thesis is a redesigned stator that should boost performance of the experimental stage from $90 \%$ adiabatic efficiency to $92 \%$ adiabatic efficiency. One reason for the increase in efficiency is the decreased loading and diffusion factor at the stator hub. The hub design is unloaded by relaxing the axial flow constraint at the stator exit. A small about of swirl (about 15 degrees) is left in the flow at the exit of the stator hub. The hub section is then less prone to separation which has a positive impact on the endwall flows since the hub endwall flows have been shown to be the leading source of loss in stage performance.

\subsubsection{Design Point Experimental Results}

The design point experiments are the core contribution of this thesis. The aspirated stage is design to produce a stage total pressure of 1.59 at an adiabatic efficiency of $89 \%$. This corresponds to a work factor $\left(\frac{\Delta H}{U^{2}}\right)$ of 0.81 . The work factor shows a significant increase over standard compressor designs which typically have work factors of 0.4-0.5.

Detailed comparisons of the pitchwise total pressure measurements from several different spanwise locations show very good agreement with the predictions. The experimental wake width matches very well with the APNASA prediction for the $28 \%, 47 \%$, and $65 \%$ span locations ( $0 \%$ is the hub, $100 \%$ is the tip) while experiment shows a larger wake at the 
$84 \%$ span location than was predicted. The rotor exit flow angle measurements also show good agreement with the predicted values for all spanwise locations. The time-accurate measurement of total pressure showed a large blade to blade variation in total pressure. A vortex shedding model was used to determine the effect of radial transport within the wake on the spanwise distribution of efficiency.

The stator exit flow conditions also compare well with the APNASA calculations. The total pressure contours measured on a plane downstream of the stator show two total pressure deficits located at the hub and tip on the suction side of the stator wake similar to the total pressure contours predicted by APNASA. In the core flow of the stator exit, the total pressure level is similar to that predicted by the APNASA solution.

The suction flow levels from the experiment are near the design values for the rotor and less than design values for the stator. For the rotor, blade span suction and passage shock suction are $0.84 \%$ of the inlet flow which is close to the design $.95 \%$ suction. In the stator, blade span suction and passage shock suction are $0.85 \%$ of the inlet flow, substantially lower than the design $1.95 \%$ suction.

\subsubsection{Off-design Results}

The aspirated stage shows several interesting results from the off-design experiments. The mass flow was decreased from design mass flow $\left(64 \frac{\mathrm{lbs}}{\mathrm{s}}\right)$ to $79 \%$ of design mass flow (50 $\frac{l b s}{s}$ ). Measurements taken from the $60 \%$ span location shows the rotor with increasingly wider wakes, from $15 \%$ of blade pitch to more than $60 \%$ of blade pitch, as the stage mass flow is reduced. The depth of the minimum total pressure ratio in the wake also decreases dramatically from 1.5 at the design mass flow rate to 1.2 at $79 \%$ of the design flow.

The APNASA and Pratt \& Whitney analysis codes predicted the off-design stage performance down to $95 \%$ design mass flow at which point the codes were unable to produce a converged solution,indicating that the stage should be approaching the stall boundary. But the off-design experiments show that the stage can operate stall-free down to a mass flow equal to $79 \%$ of the design flow. Both analyses predicted a negatively sloped speedline at mass flows less than design. The APNASA solutions predict a design pressure ratio of 1.59 
at a design mass flow of $64 \frac{l b}{s}$ and pressure ratio of 1.575 at a mass flow of $61 \frac{l b}{s}$. The Pratt \& Whitney solutions predict a design pressure ratio of 1.575 at a design mass flow of 63.8 $\frac{l b}{s}$ and a pressure ratio of 1.56 at a mass flow of $61.6 \frac{l b}{s}$. The experimental data agrees with the trends of producing lower pressure ratio at lower mass flows.

The off-design experimental data shows the rotor core flow still producing a total pressure around 1.65 down to $79 \%$ design mass flow, but the larger wakes cause the stator performance to degrade rapidly. The stage total pressure goes from 1.57 at the design point to 1.42 for the $79 \%$ mass flow case. The most unusual part of the off-design results is that the stage does not appear to enter rotating stall. The data taken downstream of the rotor and stator do not indicate the presence of rotating stall

\subsubsection{Unsteady Radial Transport}

A vortex shedding model is used to facilitate understanding of the large blade to blade variation in total pressure shown in the data. The experimental data is used to determine the model parameters which include vortex strength, vortex spacing, and vortex core size. Once the model parameters are determined, the wake velocity field induced by the vortex shedding can be determined. Using the vortex model at several spanwise locations, the spanwise pressure field within the wake can be determined. This pressure field induces radial flows which redistribute flow properties in the spanwise direction. This spanwise redistribution of flow properties is not modelled by the 3-D, viscous analysis code, APNASA.

With this in mind, the measured spanwise efficiency distribution can be 'corrected' for the unsteady radial transport. The 'corrected' efficiency distribution shows better agreement with the APNASA predictions.

\subsubsection{Parameter Study}

The preliminary parametric study provides essential insight into the compression system design regime where aspiration can have a beneficial effect on overall compressor performance. By adapting the viscous loss and diffusion factor relationship to include the effects of aspiration, the study has shown that aspiration improves both the throughflow efficiency 
and the stage pressure ratio for a large range of flow coefficients and inlet Mach numbers. The efficiency benefit of aspirated designs over non-aspirated designs gets larger as the stage pressure ratio increases. Non-aspirated designs require higher blade speeds to achieve the same stage pressure ratio as an aspirated design, and these higher blade speeds translate into higher shock losses. The parameter study also shows that aspirated designs will typically have a $100 \%$ increase in the work factor over conventional blade designs, allowing for much higher single stage pressure ratios.

The parameter study presents a simple scheme to analyze the modern compressor stage design space and highlight regions where aspiration can be most beneficial. Aspiration has the greatest effect on high blade speed compressor designs, because it controls the boundary layer downstream of the shock impingement location and presents a manageable boundary layer to the subsonic diffusion section of the blade.

\subsubsection{Stator Redesign}

The APNASA analysis of the first aspirated stage showed a $3.5 \%$ loss in the rotor and a $7.5 \%$ loss in the stator, due to an incidence mismatch. The stator has been redesigned using MISES and a 3-D, viscous analysis tool to produce several new designs that give a stage adiabatic efficiency of $92 \%$. Two separate schemes are used to decrease the stator losses. An elongation of the chord near the hub and tip controls the endwall flows better, and a relaxation of the axial exit flow constraint for the stator that leaves part of the hub swirl in the flow. The hub section has an exit swirl of 15 degrees which decreases the diffusion factor from 0.69 to 0.63 . The exit swirl is decreased linearly from 15 degrees at the hub to

0 degrees at the the $40 \%$ span location. For an engine to use this design, the flow with the excess swirl must be ingested into the core compressor. These redesigned stators along with the first rotor provide an attractive civil fan stage in terms of core efficiency and high blade loading. 


\subsection{Future Work}

For future work on the civil aspirated fan stage, an experimental investigation of the new stator design and off-design performance is needed to determine its viability as part of a civil fan stage. The detailed measurements of the off-design performance of the first aspirated stage are needed to determine the differences in the stall phenomena between aspirated and conventional fan stages.

An important future project would be to construct and test the redesigned stator with the current rotor. This set of experiments would provide a complete set of data on the performance of a fully aspirated fan stage designed to operate as the fan of a civil aircraft engine. If the experiments are successful, this new fan stage could be very attractive aerodynamically as an alternative to many commercial fan stages. Even if the aspirated stage would only break even in its impact on the overall engine performance, the aspirated fan would have the added benefit of lower blade speed. The lower blade speed translates into lower fan weight and possibly lower fan noise.

In order to improve the current aspirated stage, the endwall suction should be redesigned and minimized. With 3-D, viscous analysis tools, the full impact of endwall suction can be analyzed which would lead to more efficient suction schemes. For the current design, endwall suction comprises approximately $80 \%$ of the total stage suction. Optimizing the endwall suction requirements combined with a thorough review of the impact of the suction flows on the overall system efficiency could provide data on the optimal way to remove the suction flow from the main flowpath. Optimizing this system could make aspirated compressors more attractive for current engine application. 


\section{Appendix A}

\section{Total and Static Pressure Probe}

Figure A-1 shows the mechanical drawing used to make the total and static pressure probe. This probe is inserted into the suction channels to determine the flow total pressure and Mach number. Along with an estimate of the total temperature, the mass flow within the suction channel can be calculated. 


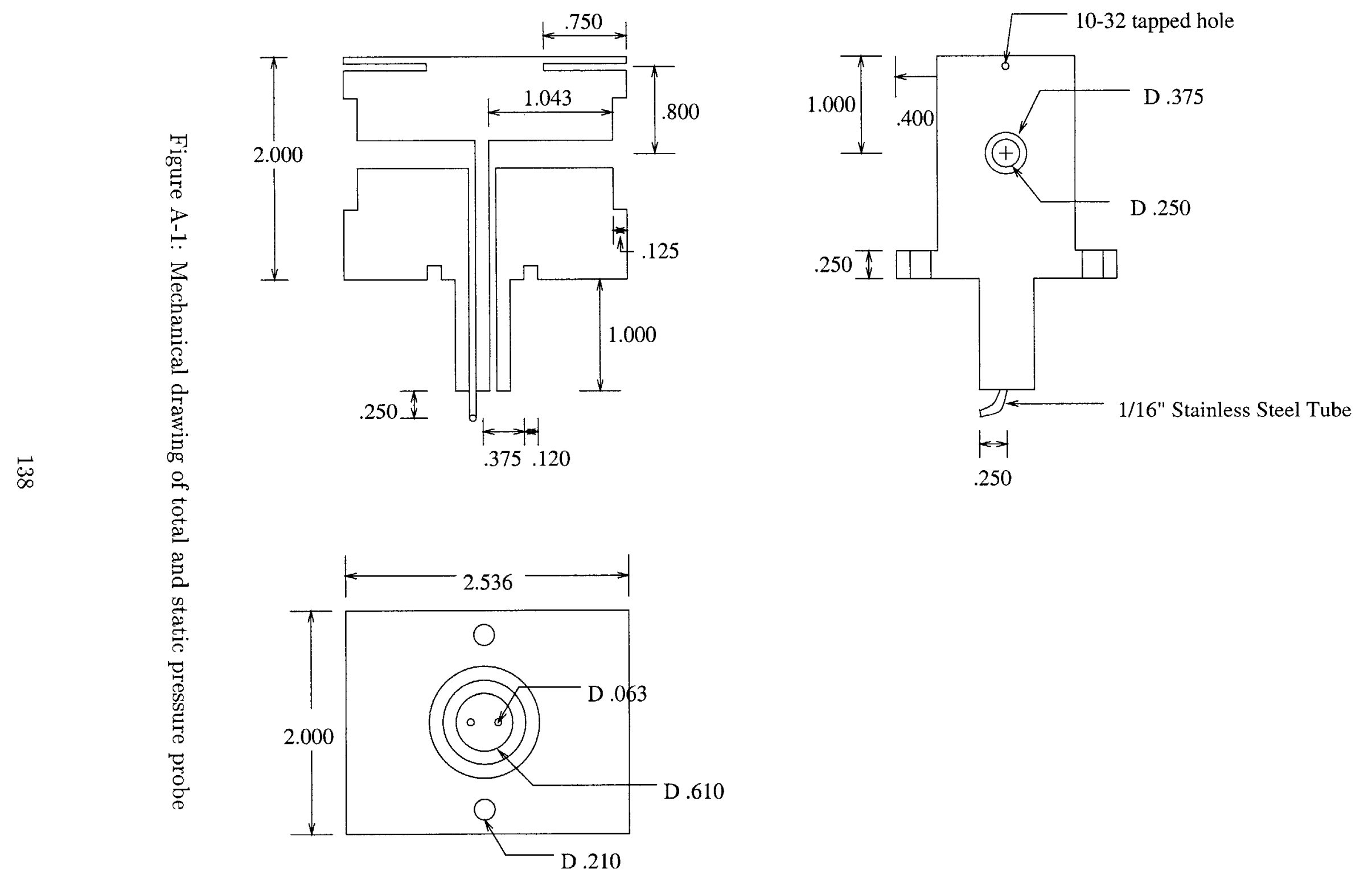




\section{Appendix B}

\section{New Blowdown Compressor Window Design}

This appendix shows the window section and plate that was designed to allow the 4-way probe to take pitchwise measurements downstream of the stator. 


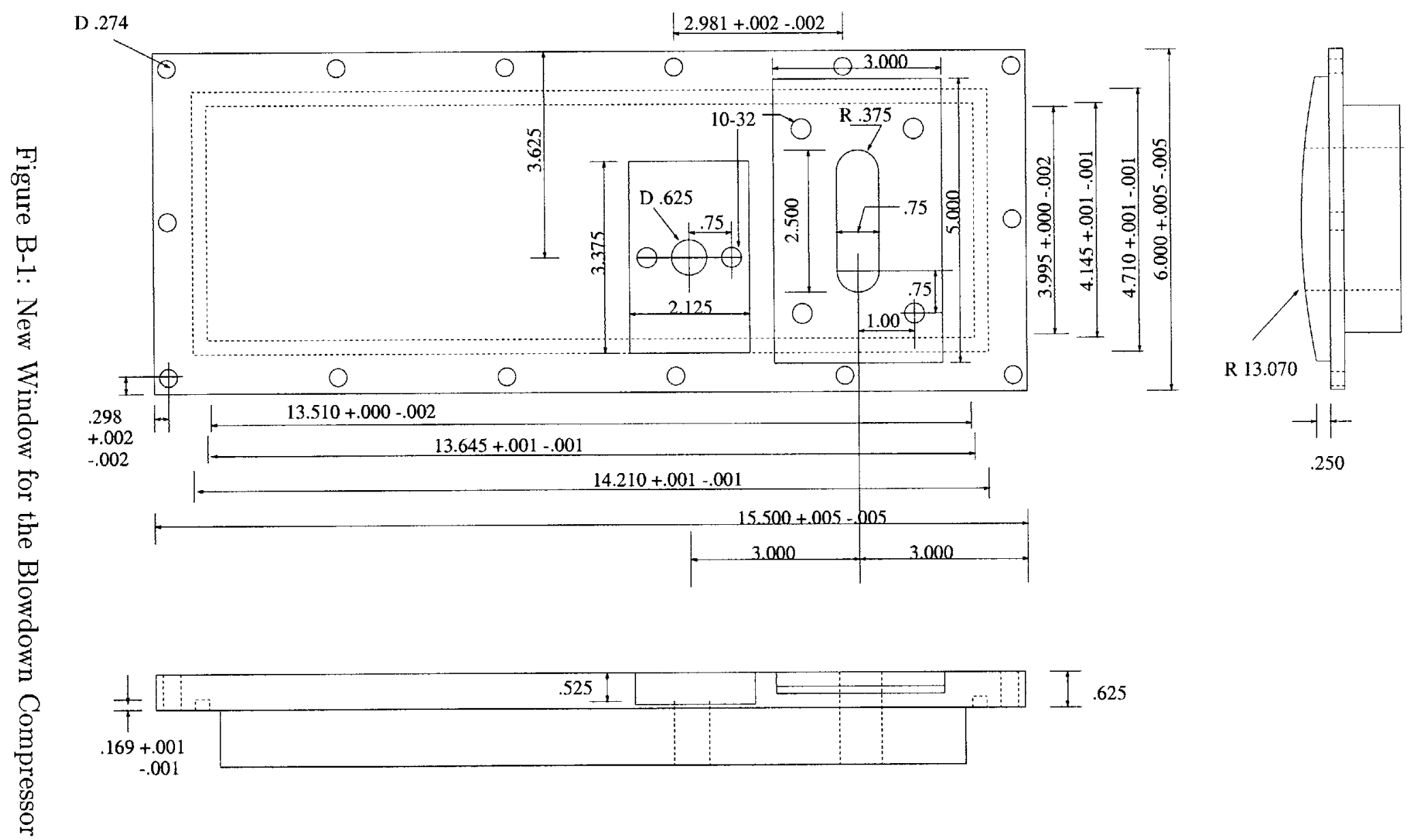

BDC Window

Brian Schuler 

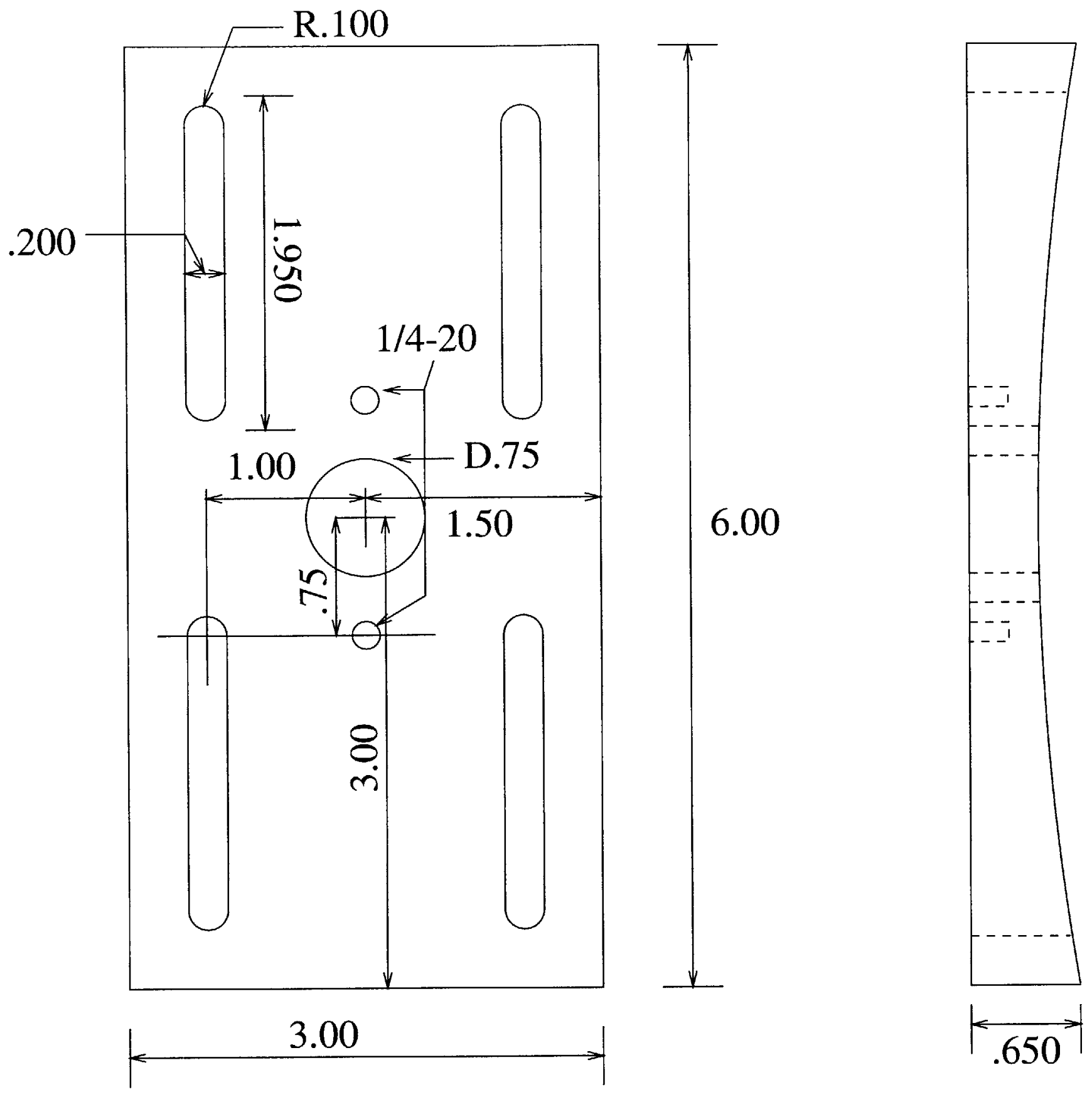

\section{BDC Slide Plate Brian Schuler}

Figure B-2: Slide Plate for BDC Window 


\section{Appendix C}

\section{4-way Probe Calibration Technique and Data}

The detailed 4-way probe calibration technique can be found in [17], but a brief description will be given here. The objective of the calibration technique is to predict total pressure, static pressure, tangential flow angle, and radial flow angle given the values of $P_{1}, P_{2}, P_{3}$ and $P_{4}$. Equations (C.1) through (C.6) show the variables used in the calibration and data reduction.

$$
\begin{array}{r}
F_{23}=\frac{P_{2}-P_{3}}{\left(P_{2}-P_{1}\right)+\left(P_{3}-P_{1}\right)} \\
C_{p 4}=\frac{P_{4}-\frac{1}{2}\left(P_{2}+P_{3}\right)}{P_{1}-\frac{1}{2}\left(P_{2}+P_{3}\right)} \\
C_{p 1}=\frac{P_{1}-P_{t}}{P_{t}-P_{s}} \\
K_{p 2}=\frac{P_{2}-P_{s}}{\left(P_{2}-P_{1}\right)+\left(P_{3}-P_{1}\right)} \\
K_{p 3}=\frac{P_{3}-P_{s}}{\left(P_{2}-P_{1}\right)+\left(P_{3}-P_{1}\right)} \\
H_{23}=\frac{P_{t}-P_{s}}{\left(P_{2}-P_{1}\right)+\left(P_{3}-P_{1}\right)}
\end{array}
$$

$F_{23}$ is the best choice for determining the tangential angle because it nondimensionalizes 
the pressure difference between transducers 2 and 3 , which is very sensitive to the tangential angle, by a first order estimate of the dynamic head $\left(P_{2}-P_{1}+P_{3}-P_{1} \approx-2 q\right)$. Furthermore, $F_{23}$ does not require any iteration because it only depends on quantities measured in the experiment. $F_{23}$ has only a small dependence on radial angle and Mach number.

$C_{p 4}$ is used in a similar way to determine the radial angle.

For a given tangential and radial angle, the remaining variables are used to determine $P_{t}$ and $P_{s}$ for a given experiment. Equation (C.7) shows the combination of variables used to determine $P_{t}$.

$$
P_{t}=P_{1}+H_{23}\left(1-C_{p}\right)\left(P_{2}-P_{1}+P_{3}-P_{1}\right)
$$

The static pressure, $P_{s}$, can be determined using either $K_{p 2}$ or $K_{p 3}$, but the best results come from an average of the two. Equations (C.8) to (C.10) shows the calculation of the static pressure.

$$
\begin{array}{r}
P_{s}^{*}=P_{2}-K_{p 2}\left(P_{2}-P_{1}+P_{3}-P_{1}\right) \\
P_{s}^{* *}=P_{3}-K_{p 3}\left(P_{2}-P_{1}+P_{3}-P_{1}\right) \\
P_{s}=\frac{1}{2}\left(P_{s}^{*}+P_{s}^{* *}\right)
\end{array}
$$

From [17], two nondimensionalized coefficients $\left(F_{23}\right.$ and $\left.C_{p 4}\right)$ can be determined based on the 4 measured pressure for a sample point during the experiment of interest. The same can be done for each data point taken during the calibration. Because the Mach number, tangential and radial angle are known during the calibration, curves can be generated for $F_{23}$ as functions of tangential angle $(\theta)$ for constant Mach number and radial angle. Similarly, curves of $C_{p 4}, C_{p 1}, K_{p 2}, K_{p 3}$ and $H_{23}$ can be generated as a function of radial angle $(\phi)$ for constant tangential angles and Mach numbers. Figures C-1 through C-7 show the calibration curves. If $M$ and $\phi$ were known during the experiment, $\theta$ could be simply obtained by interpolation using $F_{23}$ vs. $\theta$ and the known value of $F_{23}$. Because $F_{23}$ was chosen to have a minimal dependence on $M$ and $\phi$, even using a calculated guess for $M$ and $\phi$ will provide a 
very reasonable answer for $\theta$. Therefore an iteration is used to determine $\theta, \phi, P_{s}$, and $P_{t}$. So using a guess for $P_{t}, P_{s}\left(M=f\left(P_{t}, P_{s}\right)\right)$ and $\phi, \theta$ is obtained. Subsequently, the obtained $\theta$ and guessed Mach number are used when interpolating $C_{p 4}$ vs. $\phi$ and obtaining $\phi$. When $\theta, \phi$, and $M$ are known the additional four nondimensional variables can be obtained from the calibration database enabling calculation of $P_{t}$ and $P_{s}$ using equations (C.7) and (C.10). As a last step, the Mach number is recalculated based on the (new) $P_{t}$ and $P_{s}$ and is used as an input for the next iteration.

Summarizing, the following procedure is used to obtain the angles and $P_{t}$ and $P_{s}$, during a run:

1) Estimate $P_{t}$ and $P_{s}$ (Use $P_{t}=P_{1}, P_{s}$ is lowest of $P_{2}$ and $P_{3}$ )

2) Calculate total Mach number based on $P_{t}$ and $P_{s}$

3) Calculate $F_{23}$ and $C_{p 4}$

4) Start Iteration

5) Assume radial angle $(\phi)$ is zero

6) Determine $F_{23}$ as function of $\theta$ for given $\phi$ and Mach number

7) Interpolate and find $\theta$ for calculated $F_{23}$

8) Determine $C_{p 4}$ as function of $\phi$, for given $\theta$ and Mach number

9) Interpolate and find $\phi$ for calculated $C_{p 4}$

10) Determine $F_{23}$ as function fo $\theta$ for given $\phi$ and Mach number

11) Interpolate and find $\theta$ for calculated $F_{23}$

12) Determine $C_{p 4}$ as function of $\phi$ for given $\theta$ and Mach number

13) Interpolate and find $\phi$ for calculated $C_{p 4}$

14) For given $\theta, \phi$ and Mach number retrieve $C_{p 1}, K_{p 2}, K_{p 3}, H_{23}$ from database

15) Calculate new static pressure using equation (C.10)

16) Calculate new total pressure using equation (C.7)

17) Calculate new Mach number

18) Check difference between old and new static pressure, if to large goto (4)

19) Calculate components of Mach number using $\phi$ and $\theta$

The second 4-way probe was calibrated for tangential angle variation, but was unable to be calibrated for the radial angle variation. Because the probe is comstructed to be 
geometrically identical to the first probe, the $C_{p 4}$ of the first probe is used in the calibration data of the second probe. Figures C- 8 to C-14 show the calibration curves. 

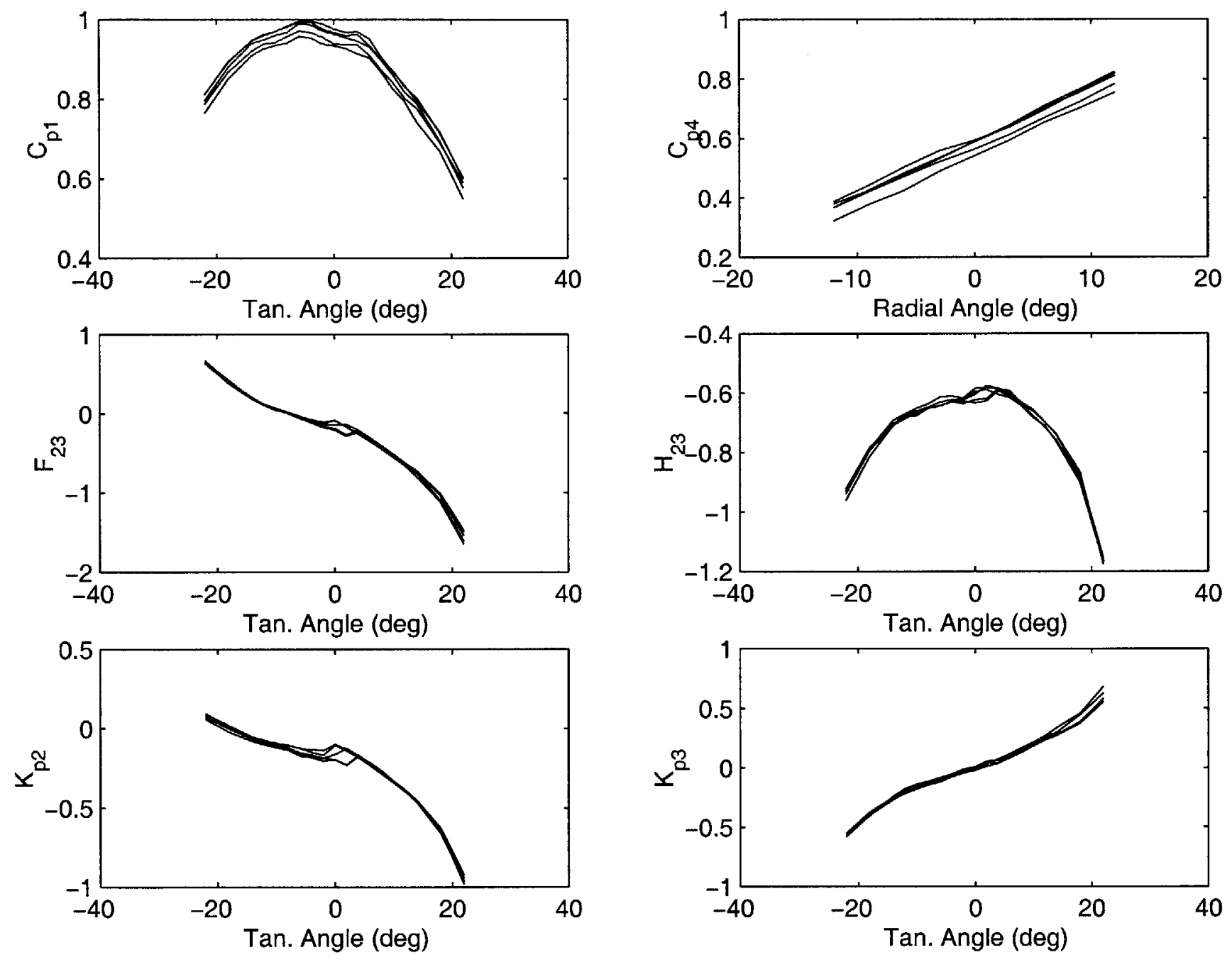

Figure C-1: $F_{23}, C_{p 4}, C_{p 1}, K_{p 2}, K_{p 3}$, and $H_{23}$ as function of $\theta$ and $\phi$ for $\mathrm{M}=0.10$ 

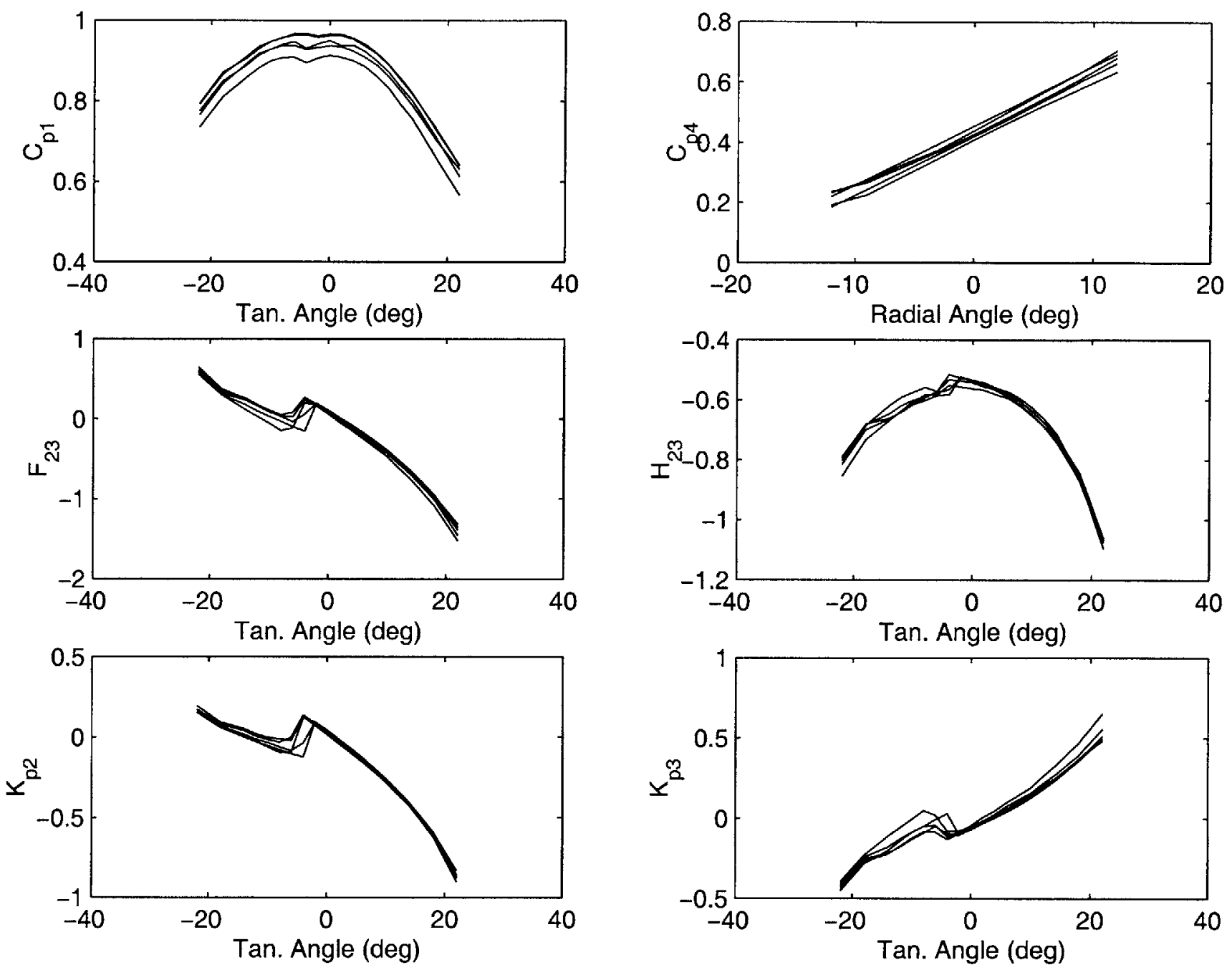

Figure C-2: $F_{23}, C_{p 4}, C_{p 1}, K_{p 2}, K_{p 3}$, and $H_{23}$ as function of $\theta$ and $\phi$ for $\mathrm{M}=0.32$ 

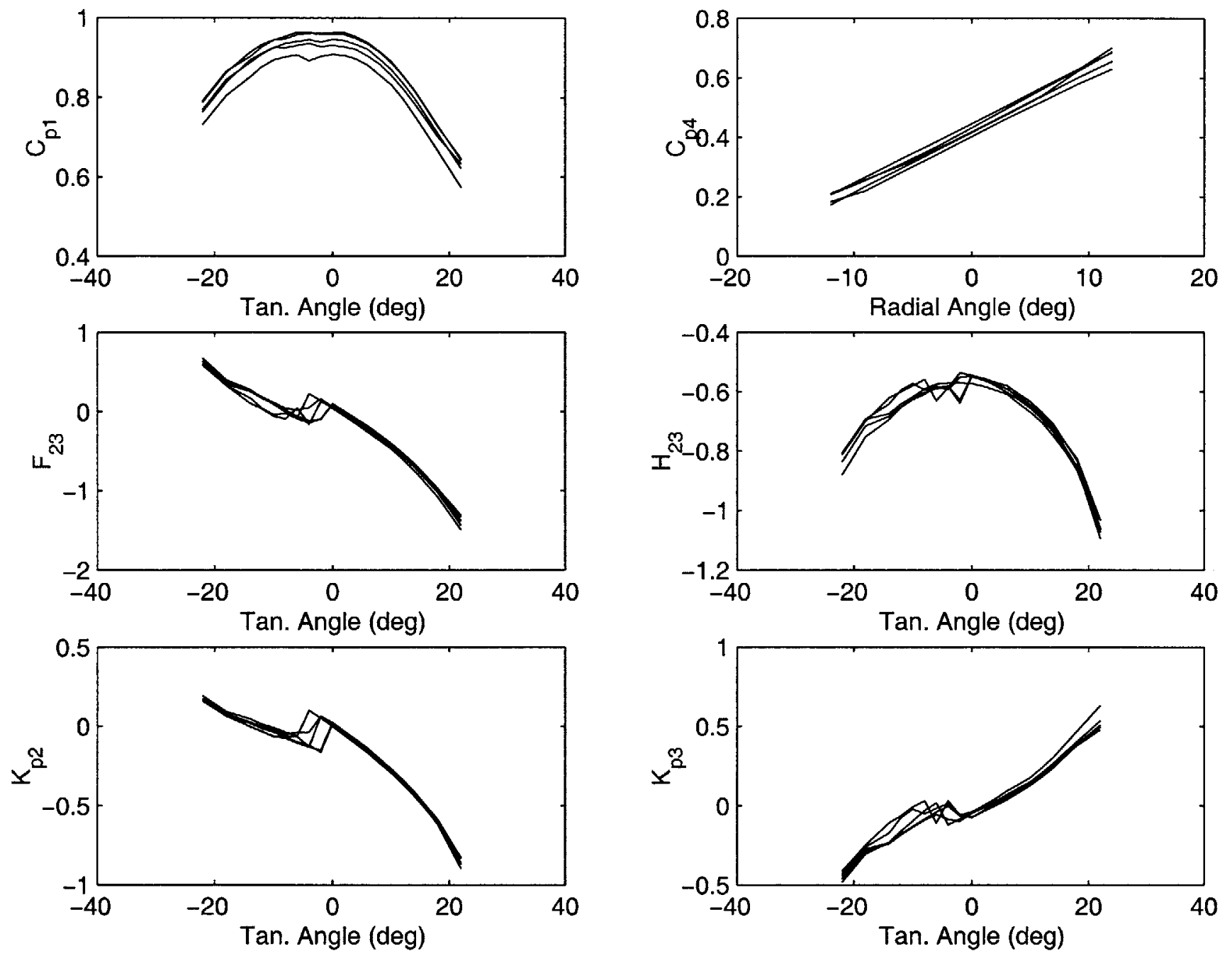

Figure C-3: $F_{23}, C_{p 4}, C_{p 1}, K_{p 2}, K_{p 3}$, and $H_{23}$ as function of $\theta$ and $\phi$ for $\mathrm{M}=0.41$ 

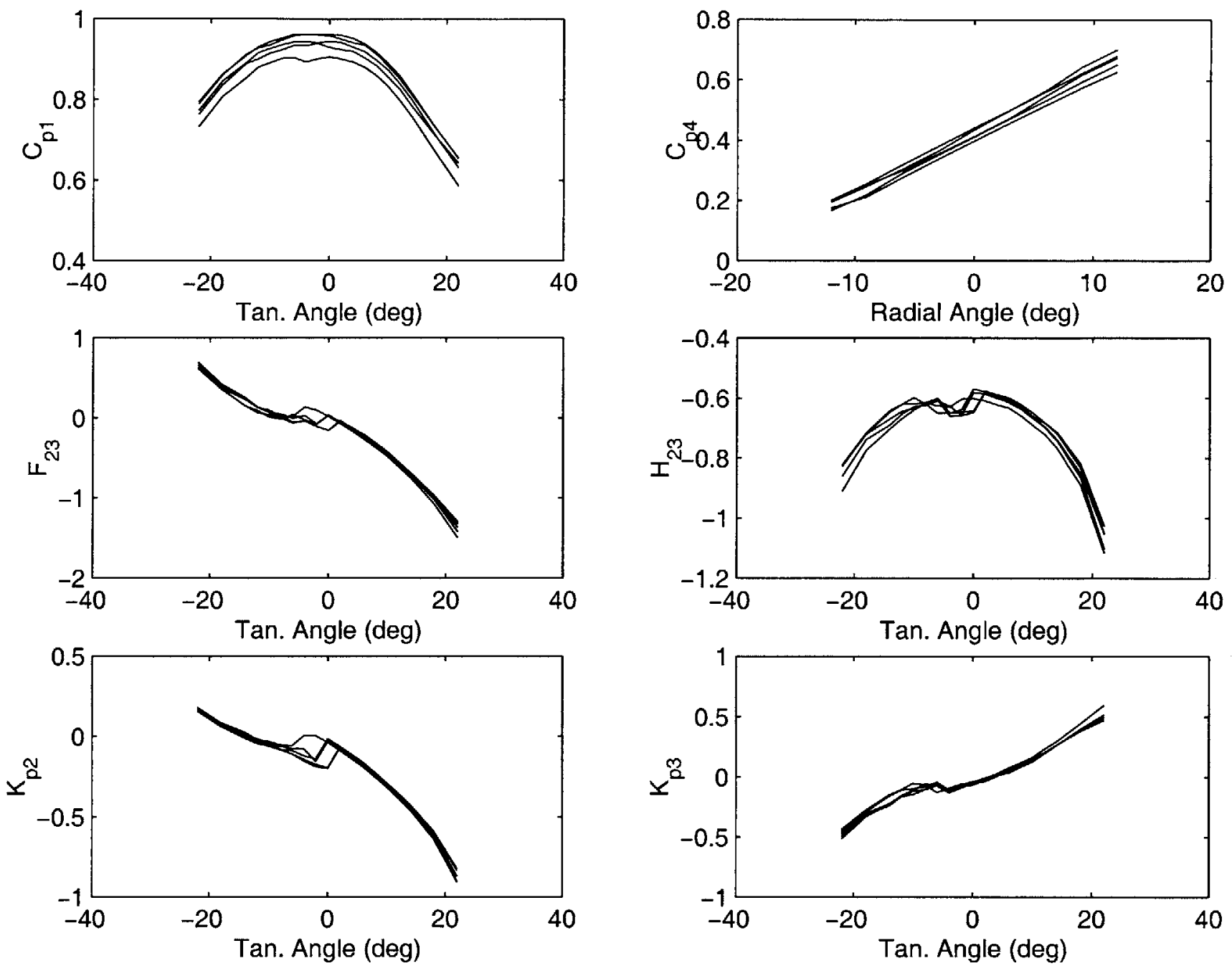

Figure C-4: $F_{23}, C_{p 4}, C_{p 1}, K_{p 2}, K_{p 3}$, and $H_{23}$ as function of $\theta$ and $\phi$ for $\mathrm{M}=0.51$ 

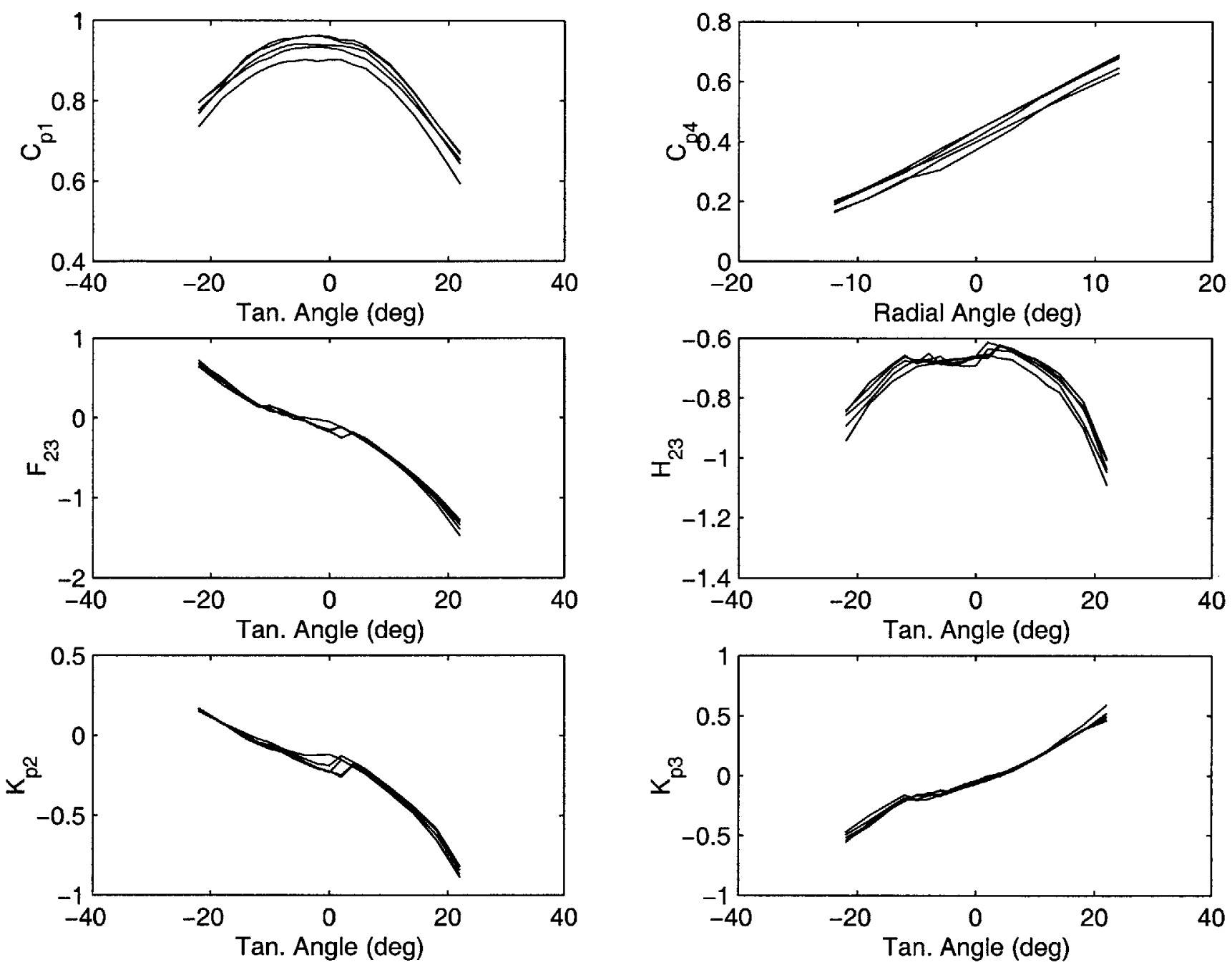

Figure C-5: $F_{23}, C_{p 4}, C_{p 1}, K_{p 2}, K_{p 3}$, and $H_{23}$ as function of $\theta$ and $\phi$ for $\mathrm{M}=0.60$ 

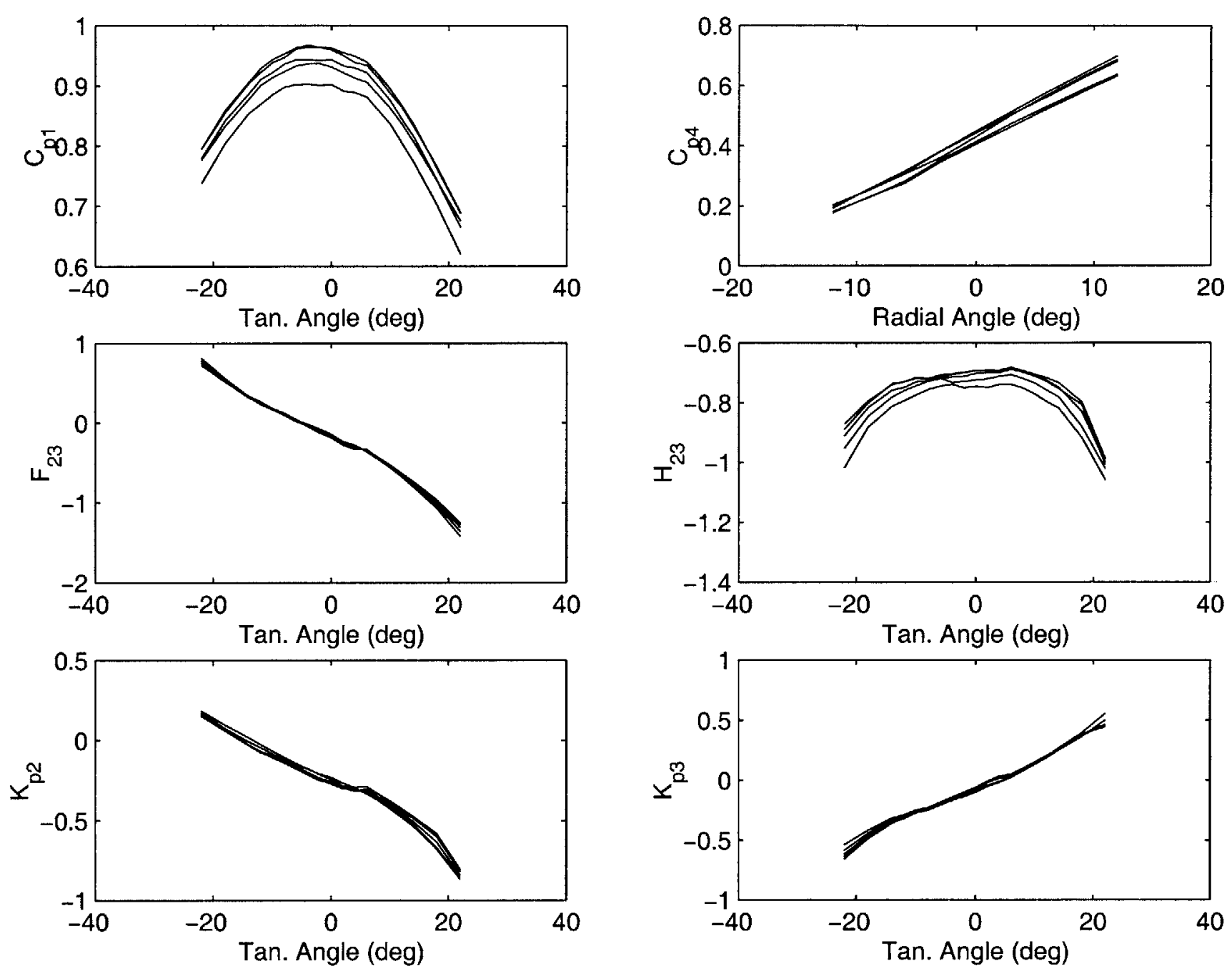

Figure C-6: $F_{23}, C_{p 4}, C_{p 1}, K_{p 2}, K_{p 3}$, and $H_{23}$ as function of $\theta$ and $\phi$ for $\mathrm{M}=0.70$ 

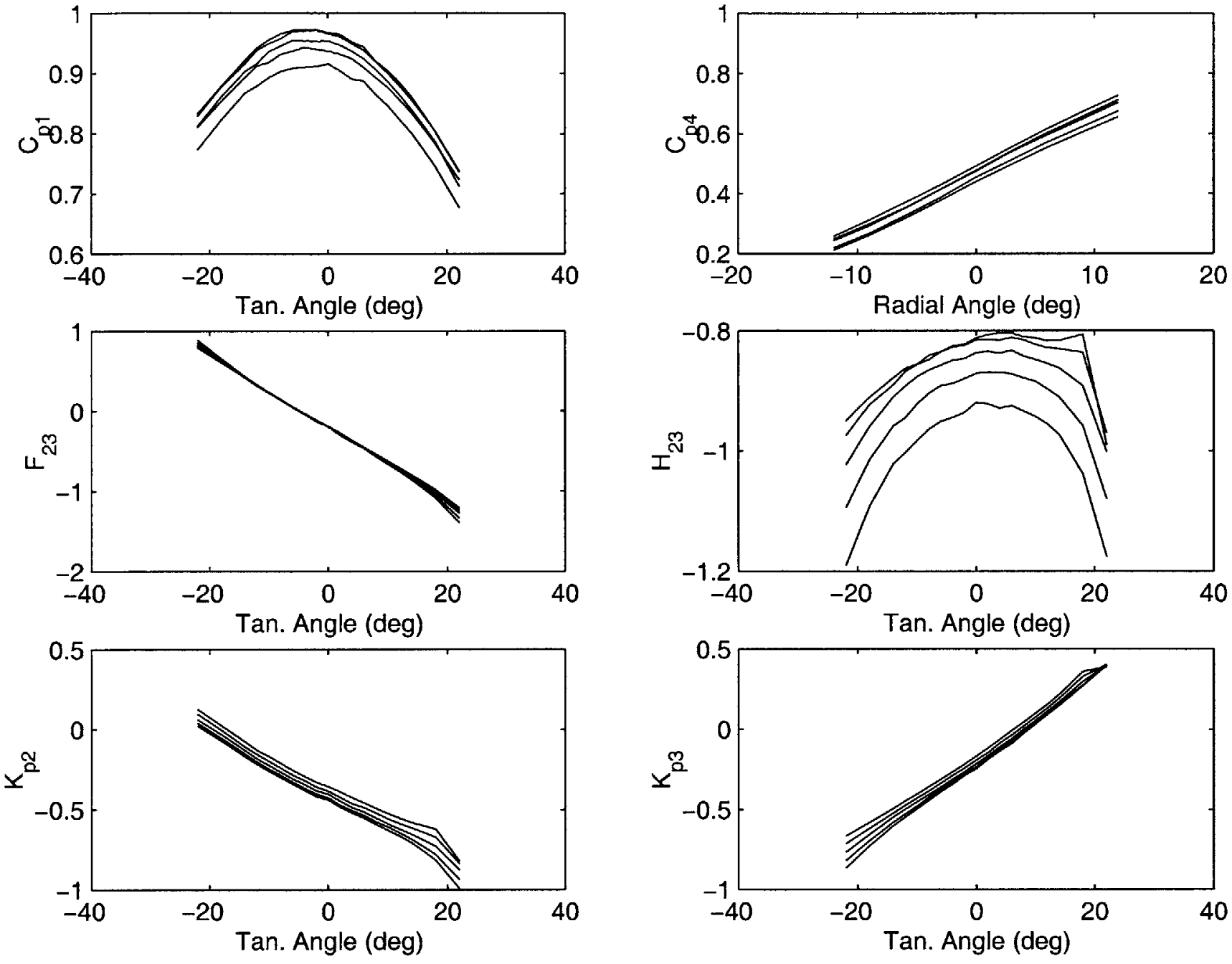

Figure C-7: $F_{23}, C_{p 4}, C_{p 1}, K_{p 2}, K_{p 3}$, and $H_{23}$ as function of $\theta$ and $\phi$ for $\mathrm{M}=0.92$ 

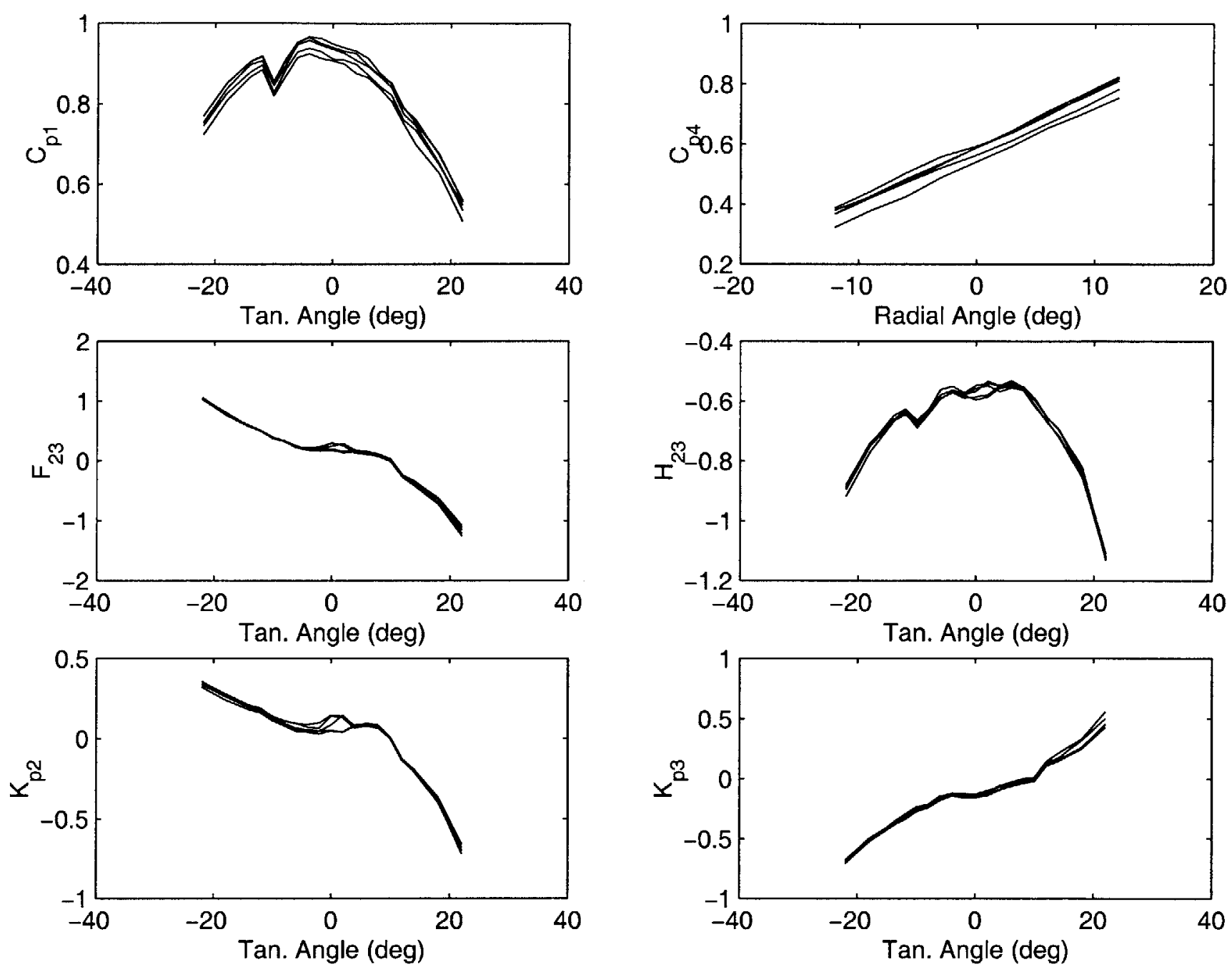

Figure C-8: $F_{23}, C_{p 4}, C_{p 1}, K_{p 2}, K_{p 3}$, and $H_{23}$ as function of $\theta$ and $\phi$ for $\mathrm{M}=0.10$ 

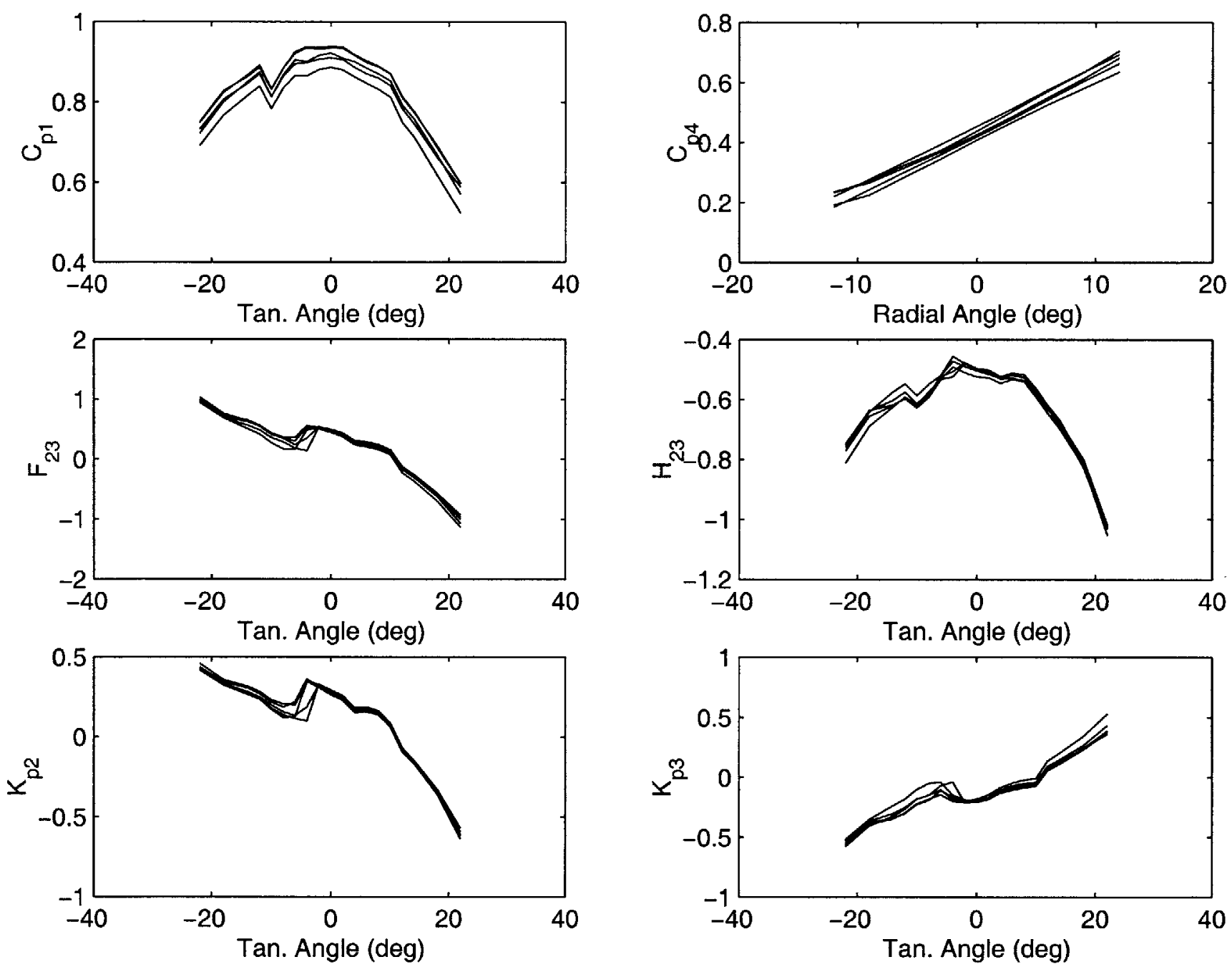

Figure C-9: $F_{23}, C_{p 4}, C_{p 1}, K_{p 2}, K_{p 3}$, and $H_{23}$ as function of $\theta$ and $\phi$ for $\mathrm{M}=0.32$ 

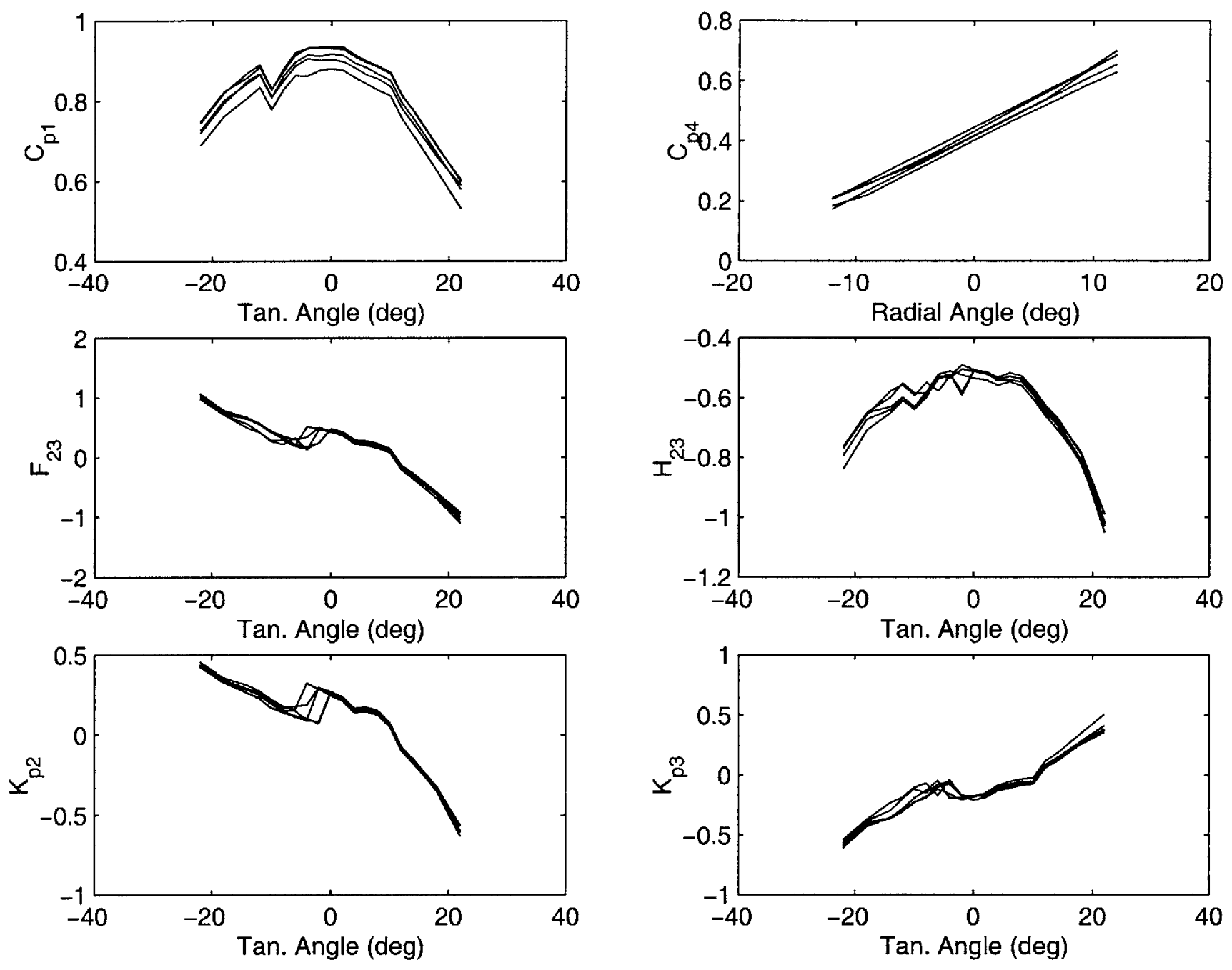

Figure C-10: $F_{23}, C_{p 4}, C_{p 1}, K_{p 2}, K_{p 3}$, and $H_{23}$ as function of $\theta$ and $\phi$ for $\mathrm{M}=0.41$ 

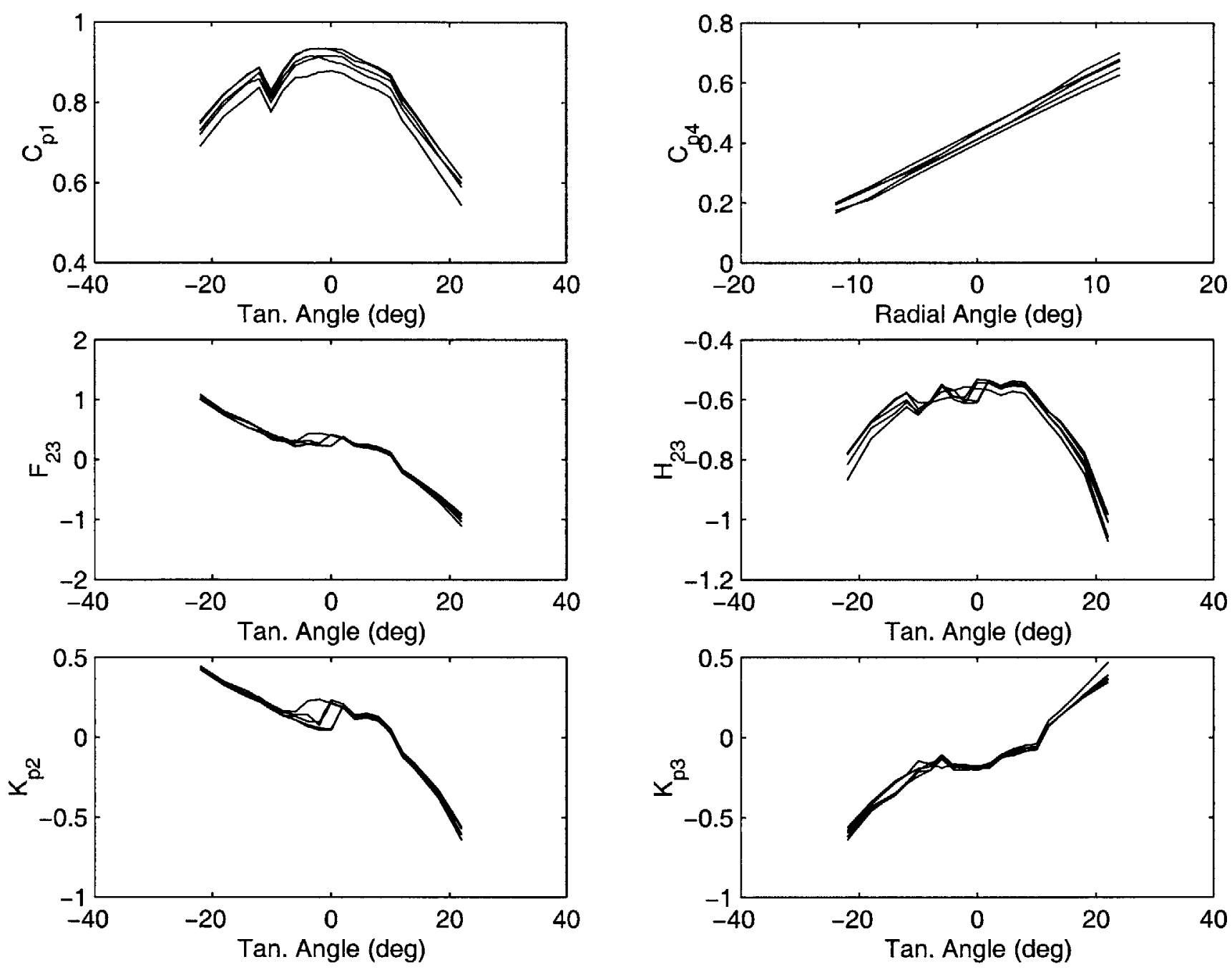

Figure C-11: $F_{23}, C_{p 4}, C_{p 1}, K_{p 2}, K_{p 3}$, and $H_{23}$ as function of $\theta$ and $\phi$ for $\mathrm{M}=0.51$ 

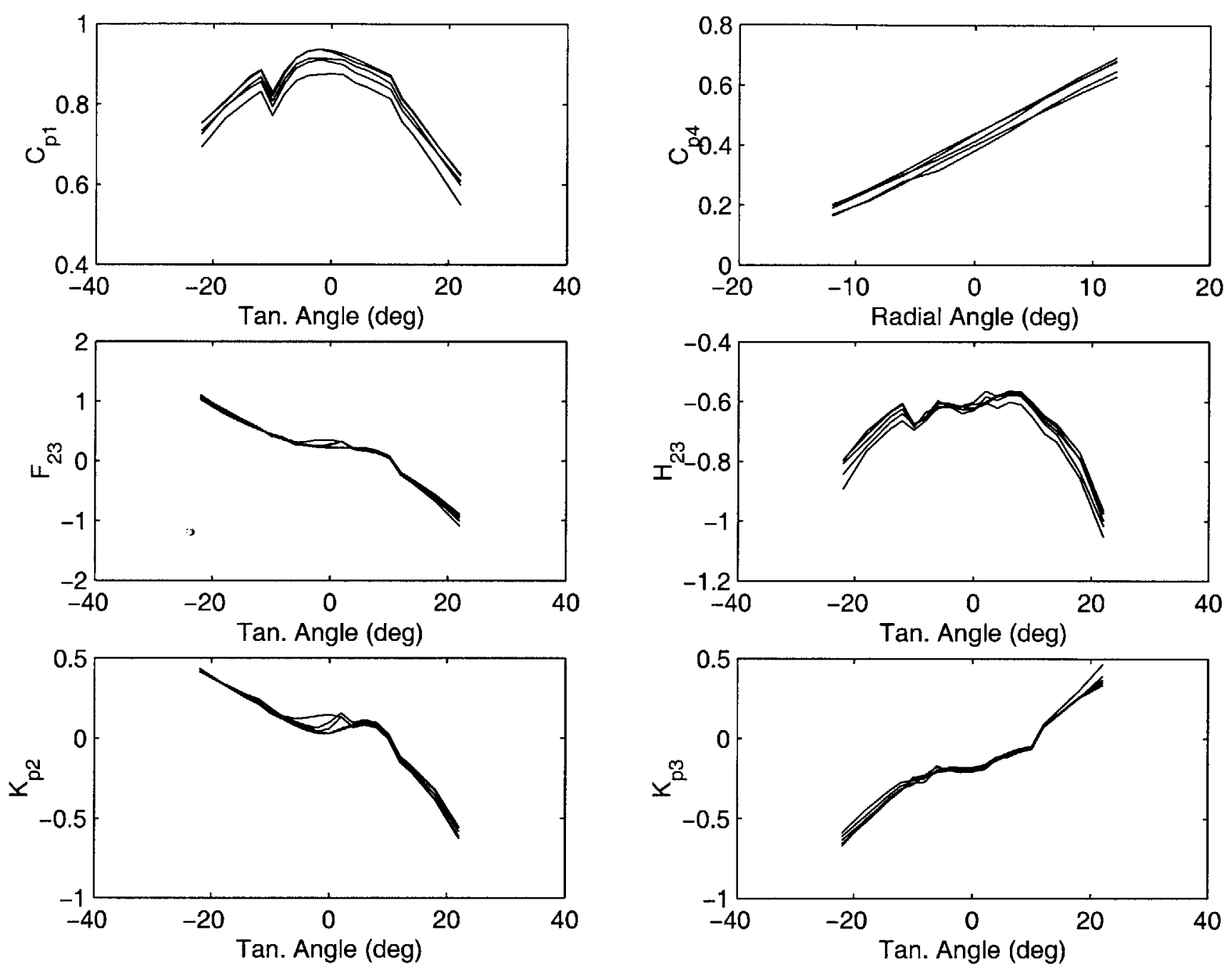

Figure C-12: $F_{23}, C_{p 4}, C_{p 1}, K_{p 2}, K_{p 3}$, and $H_{23}$ as function of $\theta$ and $\phi$ for $\mathrm{M}=0.58$ 

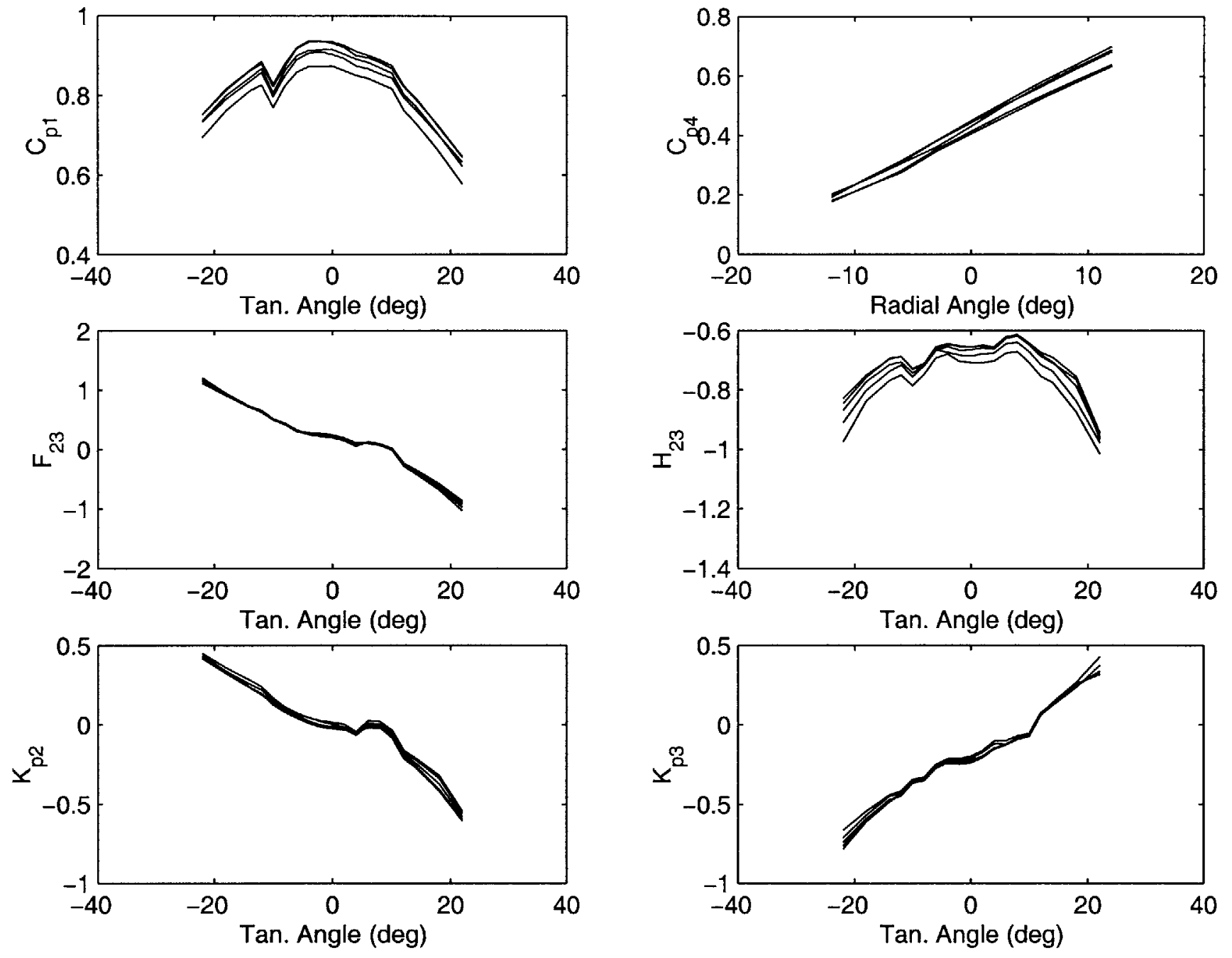

Figure C-13: $F_{23}, C_{p 4}, C_{p 1}, K_{p 2}, K_{p 3}$, and $H_{23}$ as function of $\theta$ and $\phi$ for $\mathrm{M}=0.70$ 

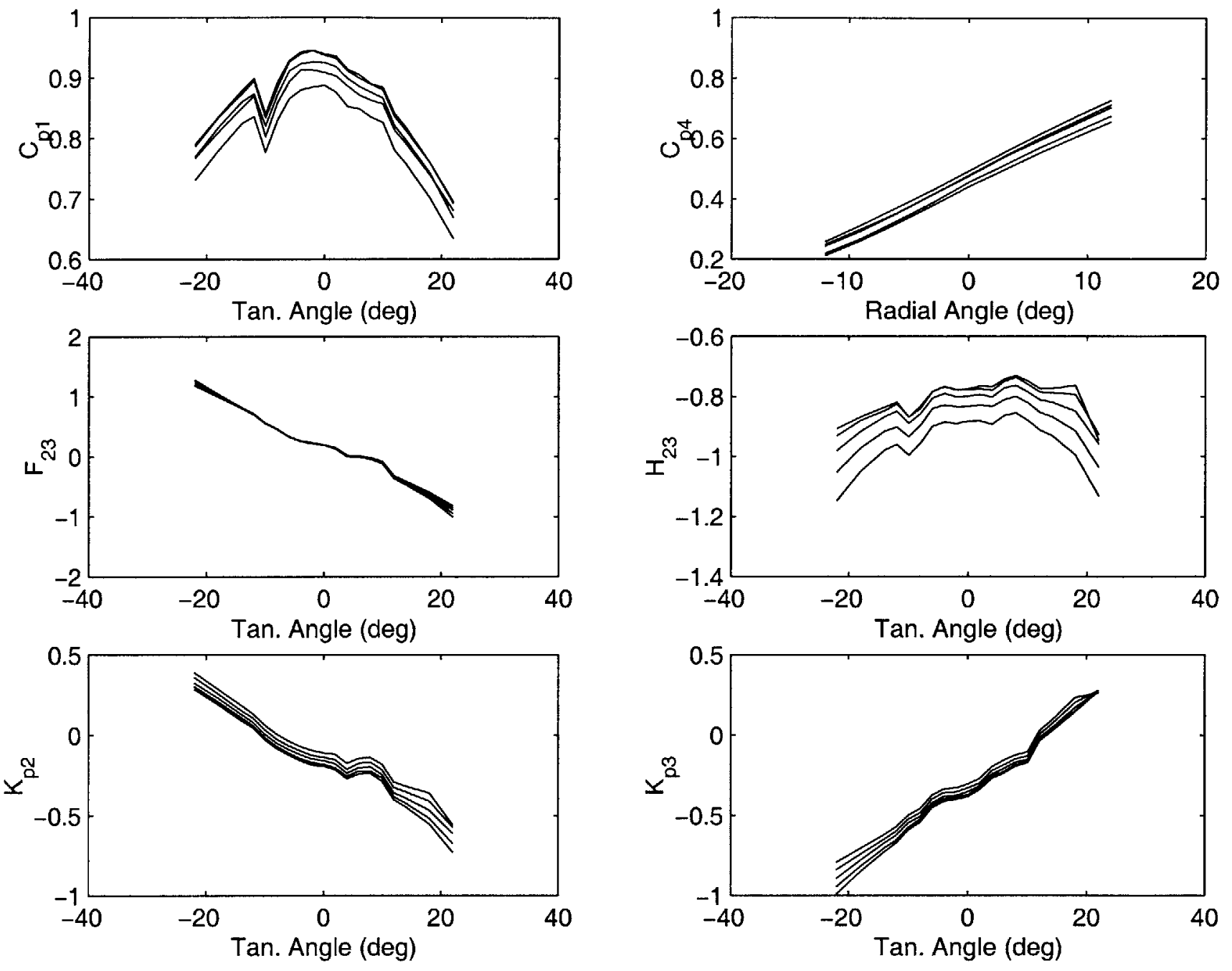

Figure C-14: $F_{23}, C_{p 4}, C_{p 1}, K_{p 2}, K_{p 3}$, and $H_{23}$ as function of $\theta$ and $\phi$ for $\mathrm{M}=0.92$ 


\section{Appendix D}

\section{Parametric Study with Radial Variation of Streamlines}

\section{D.1 Introduction}

This section of the thesis presents a more in-depth look at the effect of aspiration on the design space of fan and compressor stages by addressing the limitations of the meanline parameter study. This chapter modifies the meanline parameter study to allow the radius of the streamline to vary through the stage and presents a first-order estimate of the shockboundary layer interaction losses. Results are presented for the hub, midspan, and casing streamlines.

\section{D.1.1 Determining the Radial Variation through the Stage}

In order to determine the radial variation through the stage, some basic assumptions are needed concerning the stage efficiency, the average axial velocity through the stage, and the casing radius change through the stage. The stage efficiency is estimated using equations (D.2) and (D.3), which are empirical equations derived from the meanline parameter study where $\tau_{e s t}$ is the total temperature ratio across the rotor and given by equation (D.1). 


$$
\begin{gathered}
\tau_{e s t}=1+\frac{(\gamma-1) \phi M_{t}^{2}}{1+\frac{\gamma-1}{2} M_{1}^{2}} \\
\eta_{a d_{c} \text { onv }}=.99-.05\left(\tau_{e s t}^{\frac{\gamma}{\gamma-1}}-1\right) \\
\eta_{a d_{a} s p}=.98-.03\left(\tau_{e s t}^{\frac{\gamma}{\gamma-1}}-1\right)
\end{gathered}
$$

Once the stage efficiency and total temperature ratio are known, then the total pressure ratio can be calculated. Then three assumptions are made about the axial velocity, the casing radius, and the inlet hub to tip ratio. The axial velocity is assumed constant through the stage, the casing radius is assumed constant through the stage, and the inlet hub to tip ratio is assumed to be 0.5 . Estimates for the total and axial Mach number between the rotor and stator $\left(M_{x 2}\right.$ and $\left.M_{2}\right)$ and the stage exit Mach number $\left(M_{3}\right)$ are made by equations (D.4), (D.5), and (D.6).

$$
\begin{gathered}
M_{2 e s t}=\sqrt{M_{1}^{2}+\phi^{2} M_{t}^{2}} \\
\frac{M_{x 2 e s t}}{M_{1}}=\sqrt{\frac{1}{\tau_{e s t}} \frac{\left(1+\frac{\gamma-1}{2} M_{2 e s t}^{2}\right)}{\left(1+\frac{\gamma-1}{2} M_{1}^{2}\right)}} \\
M_{3 e s t}=\sqrt{\frac{M_{1}^{2}}{\tau_{e s t}}}
\end{gathered}
$$

Once these Mach numbers have been estimated, then constant mass flow along with 
the inlet hub to tip ratio are used to determine the hub radius change through the stage. Equations (D.7) and (D.8) show the calculation of the area ratios for the rotor exit $\left(A_{2}\right)$ and the stage exit $\left(A_{3}\right)$. Now the hub and casing radii are known throughout the stage for a given inlet Mach number, flow coefficient $(\phi)$, and work coefficient $(\psi)$. The parameter study is now calculated for the hub streamline, midspan streamline, and tip streamline.

$$
\begin{aligned}
& \frac{A_{2}}{A_{1}}=\frac{\pi_{e s t}}{\sqrt{\tau_{e s t}}} \frac{M_{2 x e s t}}{M_{1}}\left[\frac{1+\frac{\gamma-1}{2} M_{1}^{2}}{1+\frac{\gamma-1}{2} M_{2 e s t}^{2}}\right]^{\frac{\gamma+1}{2(\gamma-1)}} \\
& \frac{A_{3}}{A_{1}}=\frac{\pi_{\text {est }}}{\sqrt{\tau_{\text {est }}}} \frac{M_{3 e s t}}{M_{1}}\left[\frac{1+\frac{\gamma-1}{2} M_{1}^{2}}{1+\frac{\gamma-1}{2} M_{3 e s t}^{2}}\right]^{\frac{\gamma+1}{2(\gamma-1)}}
\end{aligned}
$$

\section{D.1.2 Parameter Study Equation Changes}

This section shows some of the major equation changes necessary to allow for radial variation of the streamlines through the stage. First, the basic parameters of flow coefficient $(\phi)$ and work coefficient $(\psi)$ are redefined as $\phi=\frac{C_{x}}{U_{t i p}}$ and $\psi=\frac{\Delta H}{U_{t i p}^{2}}$. The stage inlet velocity is nondimensionalized by the rotor tip speed, and the rotor specific enthalpy rise is nondimensionalized by the rotor tip speed squared.

The rotor diffusion factor is calculated with equation (D.9), and the stator diffusion factor is calculated with equation (D.10), where $r_{1}$ is the rotor inlet streamline radius, $r_{2}$ is the rotor exit streamline radius, $r_{3}$ is the stator exit streamline radius, $r_{t}$ is the stage tip radius (constant through the stage), $\sigma_{r}$ is the rotor solidity based on the rotor streamline inlet radius, and $\sigma_{s}$ is the stator solidity based on the stator streamline inlet radius. These equations govern the viscous loss production through the stage. The shock loss calculation does not change from the meanline parameter study.

$$
D_{R}=1-\sqrt{\frac{\psi^{2}+\left(\frac{r_{2}}{r_{t}}-\phi \frac{r_{t}}{r_{2}}\right)^{2}}{\psi^{2}+\left(\frac{r_{1}}{r_{t}}\right)^{2}}}+\frac{\phi}{\sigma_{r}\left(\frac{r_{2}}{r_{t}}+\frac{r_{1}}{r_{t}}\right)\left(\psi^{2}+\frac{r_{1}^{2}}{r_{t}^{2}}\right)^{\frac{1}{2}}}
$$




$$
D_{S}=1-\frac{M_{x 2}}{M_{2}}+\frac{\phi \frac{r_{t}}{r_{2}} M_{t} \frac{T_{1}}{T_{2}}}{\sigma_{s} M_{2}\left(\frac{r_{3}}{r_{t}}+\frac{r_{2}}{r_{t}}\right)}
$$

In addition to the radial streamline variations through the stage, the effect of the passage shock on the axial velocity ratios for the both the rotor and stator are accounted for in the rotor and stator diffusion factors. The addition of this effect to the parameter study attempts to allow the shock strength to affect the viscous losses in such a way as to globally account for the interaction between the shock and the blade boundary layer [12].

The justification for this approach is that the axial velocity ratio (AVR) across a blade row for a specific streamline is strongly affected by the passage shock. Figure D-1 shows that for a 2-D, uncambered airfoil with a passage shock, the AVR for the blade is equal to the velocity ratio across the shock. The rotor and stator diffusion factors, and hence the viscous losses of the blades, are significantly increased by decreasing AVR.

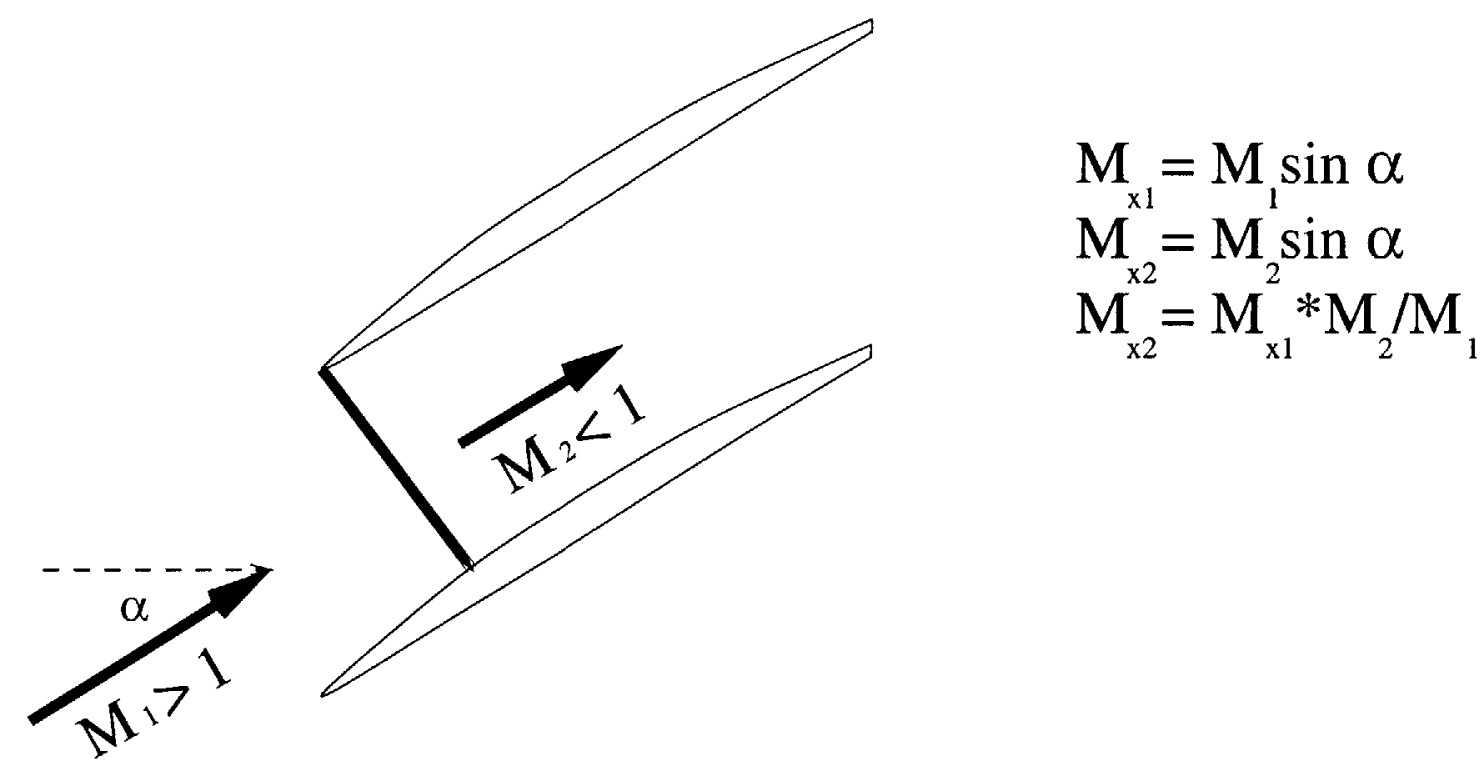

Figure D-1: Diagram of an uncambered, 2-D airfoil with passage shock

The addition of the AVR to the parameter study is intended solely as a first order attempt to capture some of the effects of the shock-boundary layer interaction to get more realistic estimates of the blade row efficiency. The shortcomings of this approach to calculating the 
AVR are first, that it does not consider the streamtube contraction through the stage which would tend to increase the axial velocity ratio, and second, that the airfoils considered in this parameter study are far from uncambered.

\section{D.1.3 Comparison Plots of Parameter Study with Compressor Data}

In this section, comparisons are made between the parameter study and compressor data. In order to have accurate comparisons, the parameter study variables are set to the values shown in table (D.1) for the given comparison. Because the NASA and Wennerstrom stages operate at nearly the same inlet flow conditions, wheel speed, and hub-to-tip ratio, only one line is calculated for those two sets of compressor data. The parameter study uses these parameters and allows the work coefficient $(\psi)$ to vary from 0.2 to 0.9 to achieve the comparison plots.

Table D.1: Parameter study values used to create comparison plots.

\begin{tabular}{||l|c|c|c|c|c|}
\hline & $\phi$ & $\sigma_{\text {rtip }}$ & $\sigma_{\text {stip }}$ & $M_{x}$ & $\frac{h u b}{t i p}$ \\
\hline Low Speed Aspirated & .890 & 1.4 & 1.5 & .64 & .59 \\
\hline High Speed Aspirated & .475 & 2.0 & 2.0 & .65 & .40 \\
\hline Conventional Stage & .426 & 1.5 & 1.6 & .58 & .38 \\
\hline
\end{tabular}

Figures D-2 and D-3 compare the results of the parameter study that includes radial variations with compressor data. Figure D-2 shows that the parameter study estimations of the stator inlet Mach number on the tip streamline compared to two aspirated designs and two conventional designs. The parameter study is very accurate for the high speed aspirated design and the Wennerstrom stage data. The parameter study slightly underpredicts the Mach number for the low speed aspirated stage and the NASA stage.

Figure D-3 compares the parameter study results of the stator inlet Mach number for the hub streamline. Here again, the parameter study overpredicts the value for the low speed aspirated stage, the NASA stage, and the Wennerstrom stage. The stator Mach number is overpredicted for the high speed aspirated stage. 


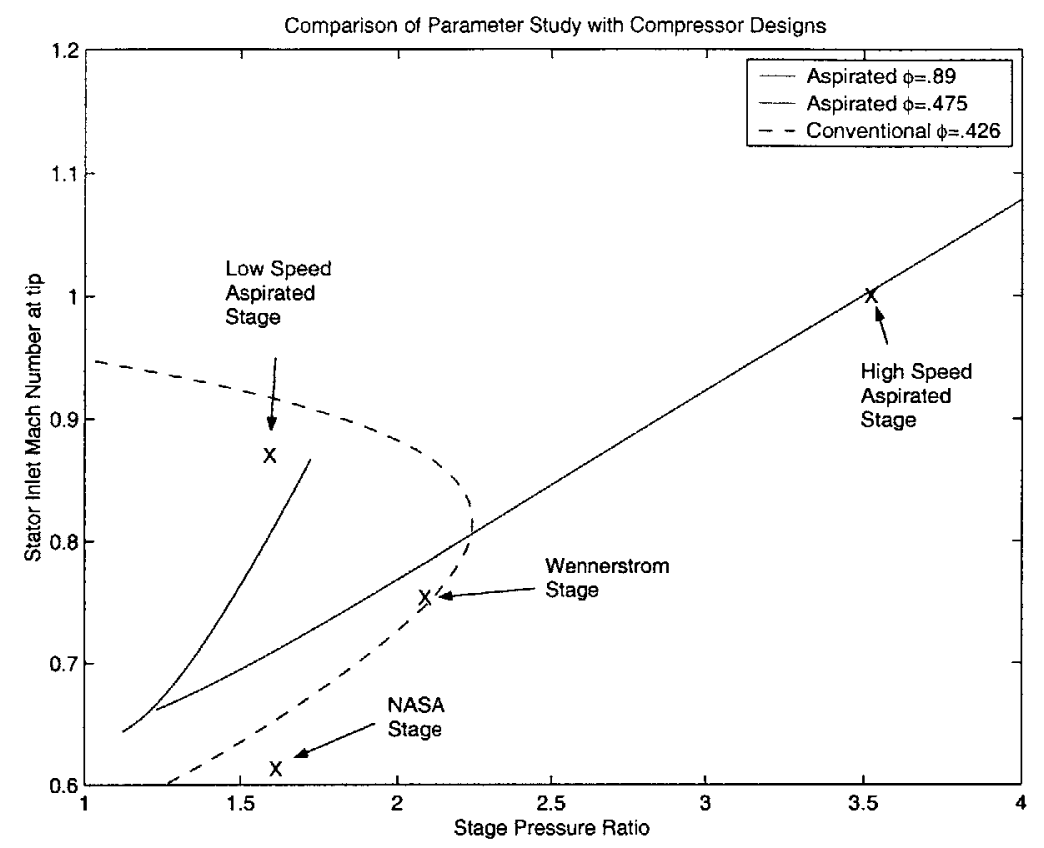

Figure D-2: Stator Inlet Mach Number Comparison of Parameter Study with Compressor Data at tip section

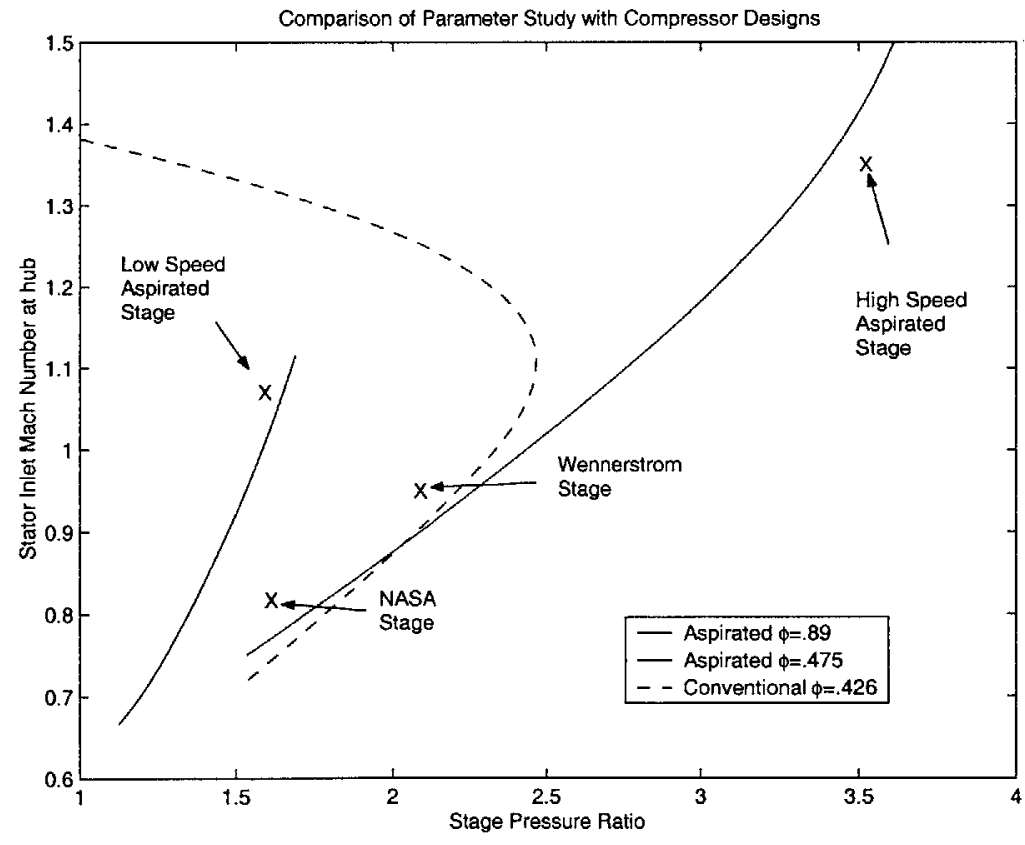

Figure D-3: Stator Inlet Mach Number Comparison of Parameter Study with Compressor Data at hub section 
Figure D-4 compares the computed diffusion factor at the rotor tip section with compressor data. The predicted diffusion factors are in good agreement to the compressor data. The parameter study overpredicts the diffusion factor for the high speed aspirated design and the Wennerstrom stage, while the diffusion factors for the low speed aspirated stage and the NASA stage are underpredicted.

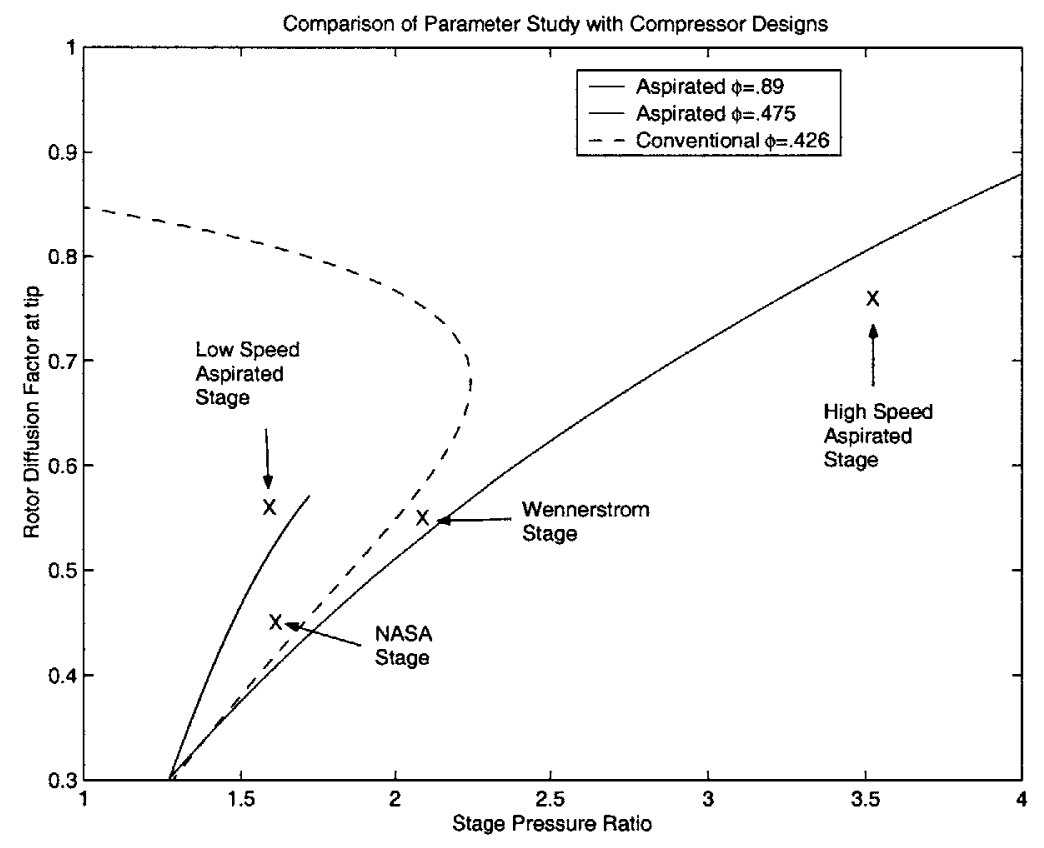

Figure D-4: Rotor Diffusion Factor Comparison of Parameter Study with Compressor Data at tip section

These comparisons of the parameter study results with both aspirated and conventional compressor designs show that the parameter study does a good job of predicting the trends and magnitude of important parameters used in compressor design. This parameter study could be used as a first step in predicting the inlet and exit flow angles for the rotor and stator, viscous and shock losses, diffusion factors, and radial streamline variation necessary to make an accurate preliminary design of either a conventional or aspirated compressor.

\section{D.1.4 Results with Radial Variations and Axial Velocity Ratio}

This section presents results of the parameter study that include effects of radial variation and the axial velocity ratio through the stage. Because of the large number of variables in the study, the inlet rotor face Mach number $\left(M_{x}\right)$ is set to 0.65 , and the flow coefficient 
is set to 0.475 and 0.89 , two values representing the two designs proposed by Merchant in reference [14].

Figures (D-5), (D-6), and (D-7) compare the design space of aspirated and conventional compressors for hub, midspan, and tip streamlines.

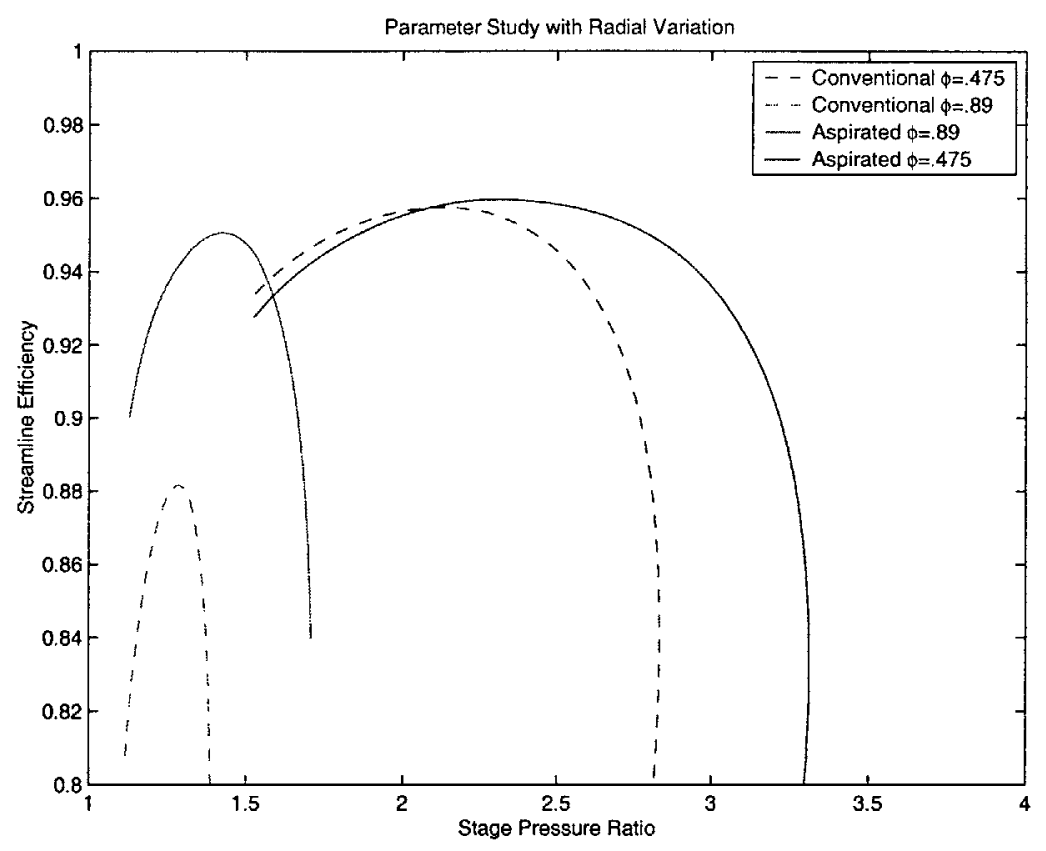

Figure D-5: Hub Streamline for conventional and aspirated fan

Figure (D-5) shows the maximum pressure ratio that can be achieved by a conventional design for $\phi=.89$ at the hub streamline is 1.55 , and the maximum efficiency of $92 \%$ is achieved for a stage pressure ratio of 1.4. For the aspirated designs, the maximum throughflow efficiency is $94 \%$ at a pressure ratio of 1.5 . For $\phi=.475$, the differences between conventional and aspirated designs are more dramatic. The maximum pressure ratio achieved is 3.0 while the maximum efficiency is $95 \%$ for a pressure ratio of 2.5 . For an aspirated design philosophy, the hub streamline has a maximum efficiency of $97 \%$ at a pressure ratio of 2.7 , but the streamline efficiency remains above $95 \%$ up to a pressure ratio of 4.0 .

For a flow coefficient of $\phi=.89$, figure (D-6) shows the maximum efficiency achieved by the midspan streamline is $95 \%$ for a pressure ratio of 1.55 . For the aspirated designs, the maximum efficiency achieved is $96 \%$ at a pressure ratio of 1.65 . For the lower flow coefficient of $\phi=.475$, the maximum pressure ratio achieved by the midspan streamline for a conventional design is 2.9 while the maximum efficiency is $90 \%$ at a pressure ratio of 2.3 . 


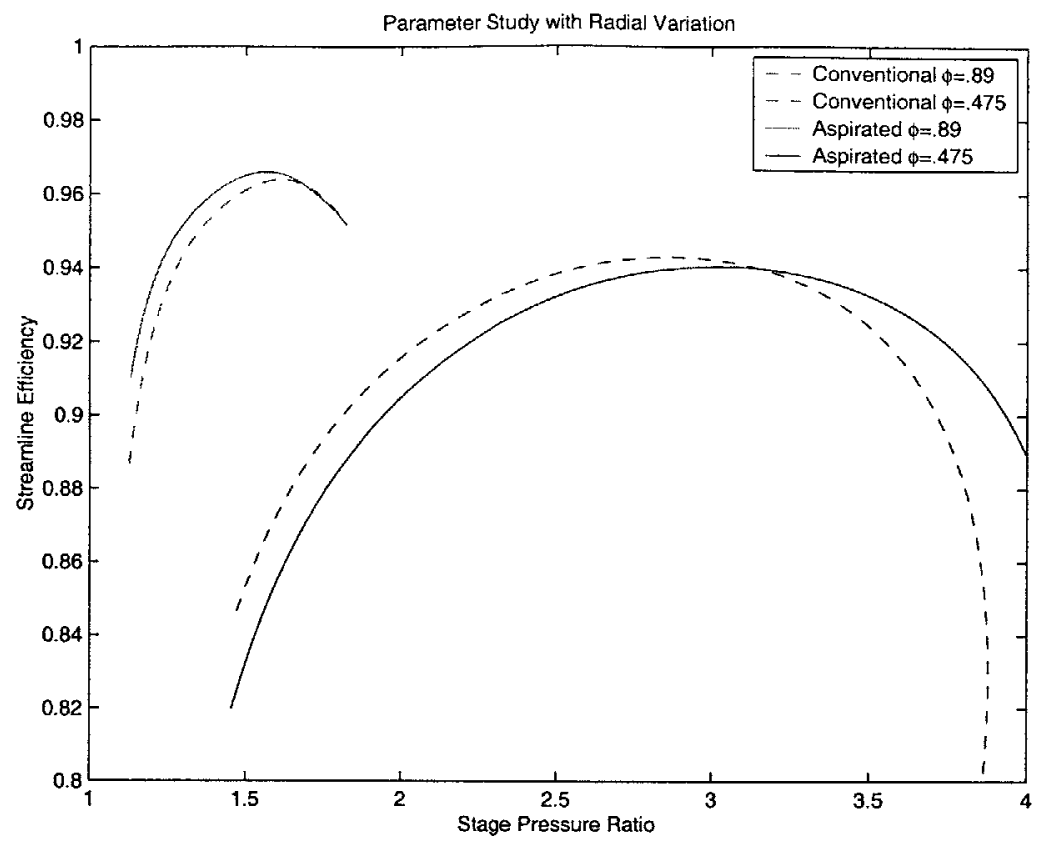

Figure D-6: Midspan Streamline for conventional and aspirated fan

For an aspirated design, the midspan streamline shows a maximum efficiency of $96 \%$ for a pressure ratio of 3.5 to 4.0 .

For a flow coefficient of $\phi=.89$, figure (D-7) shows the maximum efficiency achieved by the casing streamline is $95 \%$ for a pressure ratio of 1.7 . For the aspirated designs, the maximum efficiency achieved is $96 \%$ at a pressure ratio of 1.75 . For the flow coefficient of $\phi=.475$, the maximum pressure ratio achieved by a conventional design at the casing streamline is 2.2. The maximum efficiency achieved by the casing streamline is $72 \%$ at a pressure ratio of 2.0. For an aspirated design, the casing streamline shows a maximum efficiency of $91 \%$ for a pressure ratio of 4.0 . The effect of the shock-boundary layer interaction on the losses can really be seen in the high speed conventional design. The only difference between the high speed aspirated design and the conventional design is the viscous losses of the rotor and stator. The stator viscous losses at the tip are small compared to the rotor tip. Therefore, the rotor tip section viscous losses dominate the performance of the tip streamline. Aspiration decreases the viscous losses by a large amount for the aspirated design compared to the conventional design.

To produce a preliminary compressor stage design, all of the radial streamlines must be considered, and this study has presented the performance of hub, midspan, and casing 


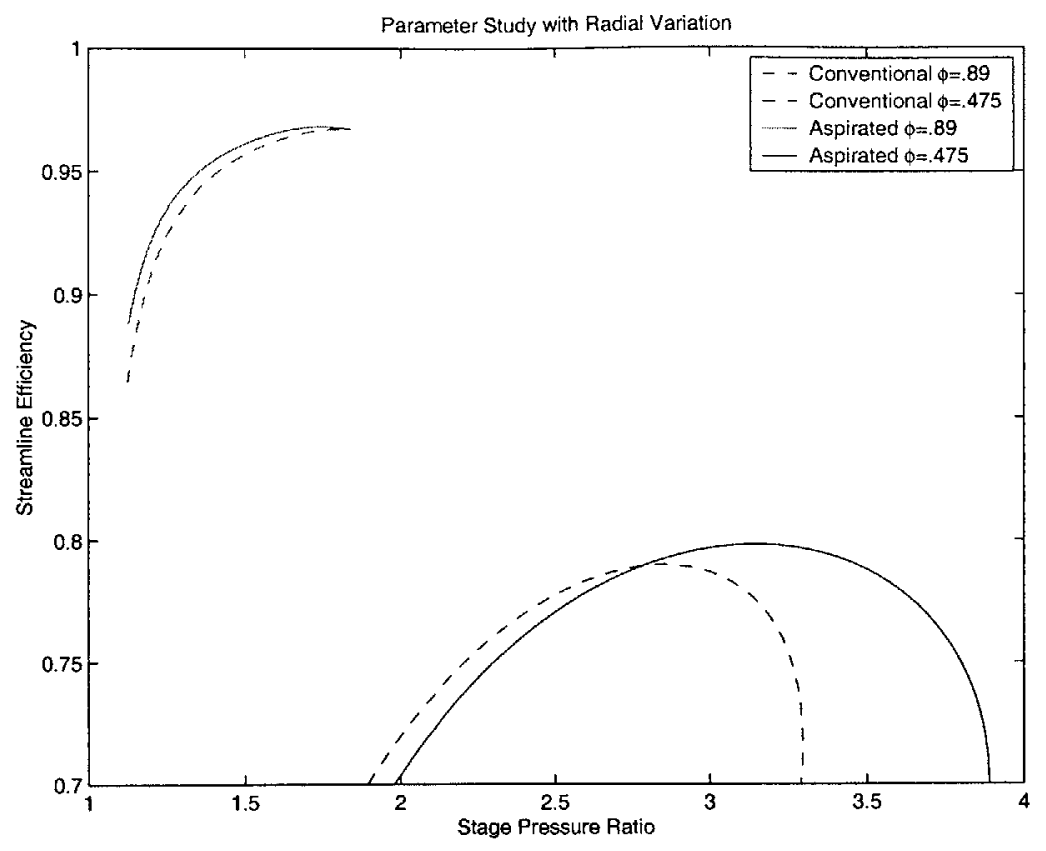

Figure D-7: Tip Streamline for conventional and aspirated fan

streamlines for both conventional and aspirated compressor designs. For a low speed design, the hub streamline becomes the limiting section for stage performance. For a conventional set of blade rows and a free vortex design, the hub section sets the maximum pressure ratio that the stage can achieve. For a flow coefficient of $\phi=.89$ and an inlet axial Mach number of 0.65 , this maximum pressure ratio is 1.55 . For aspirated, free vortex designs at high flow coefficients, the hub streamline also limits the maximum pressure ratio that can be achieved although this limit is significantly higher than for conventional designs. For higher flow coefficients and a free vortex design, the tip streamline becomes the most important sections of the blade design. For inlet axial Mach numbers on the order of 0.6, the relative Mach number into the rotor tip section is much greater than 1.0. This large relative Mach number produces a large passage shock that impinges on the blade boundary layer and significantly degrades its performance. Aspiration controls the growth of the boundary layer downstream of the shock impingement location allowing for much better throughflow efficiency and stage pressure rise. The study shows that aspiration can give a $20 \%$ increase in throughflow stage efficiency and a doubling of the stage pressure ratio for the tip streamline.

Finally, rotor and stator diffusion factors for both the hub and tip radii are shown, along with the stator inlet Mach number for hub and tip, and the rotor reaction for both 
streamlines.

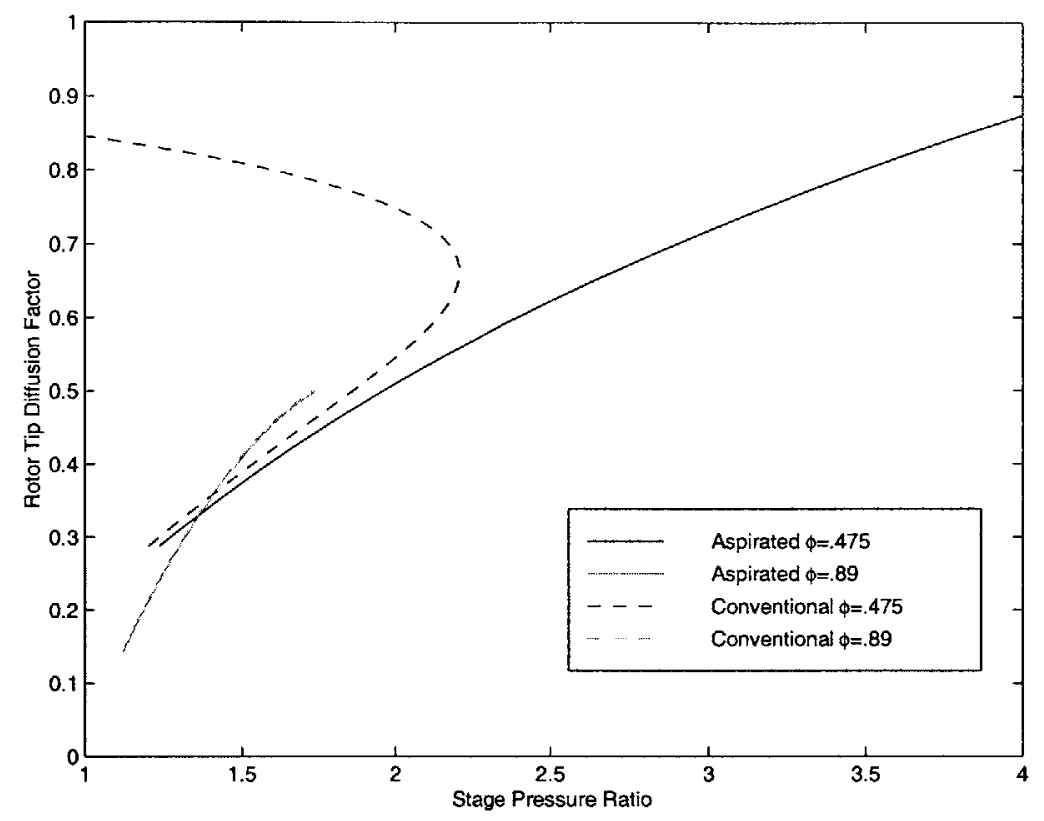

Figure D-8: Rotor Diffusion Factor for the Tip Section 


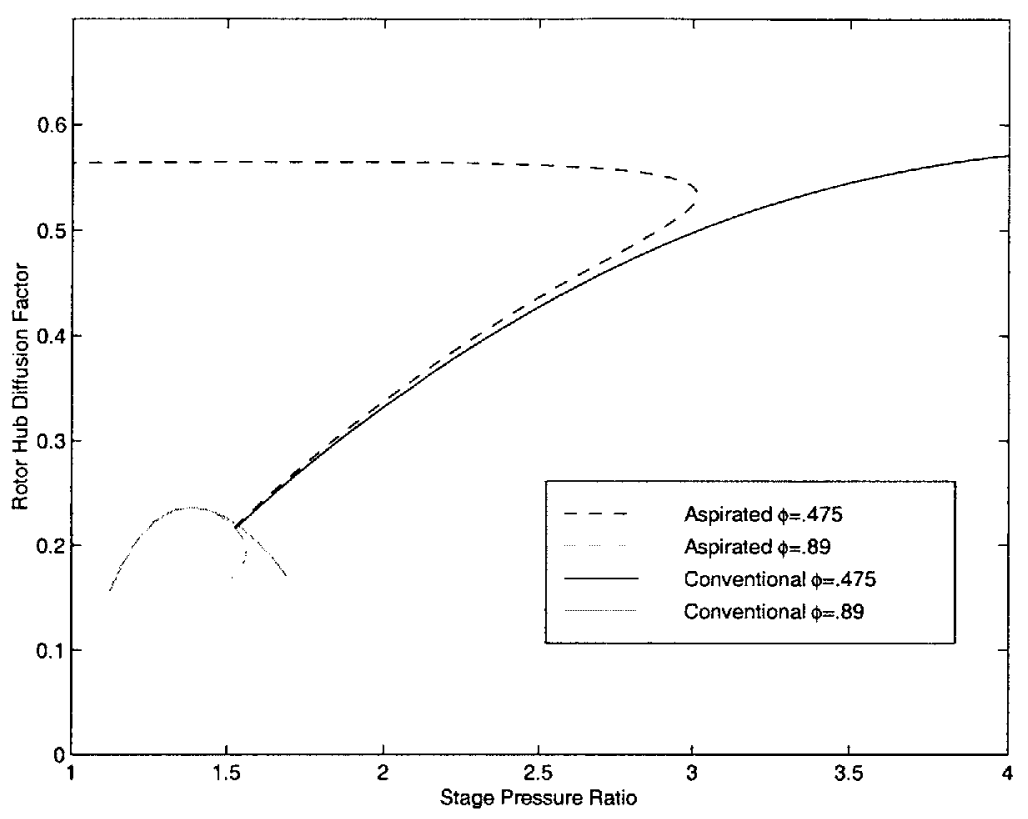

Figure D-9: Rotor Diffusion Factor for the Hub Section

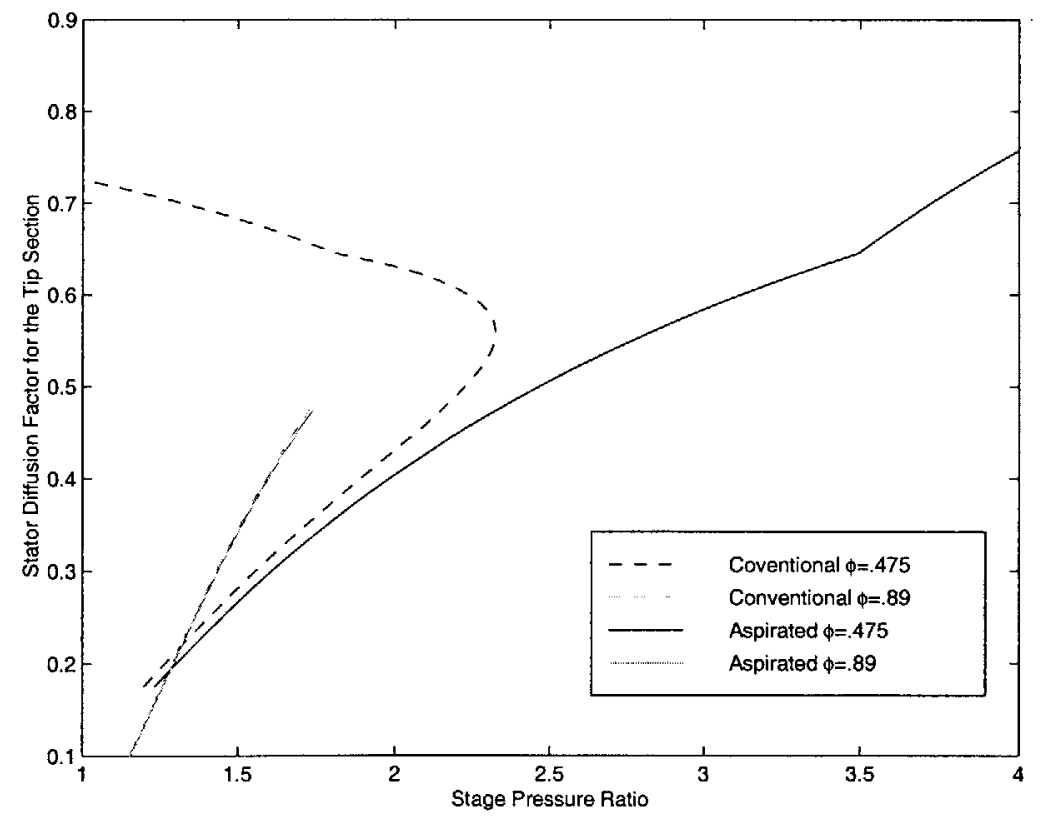

Figure D-10: Stator Diffusion Factor for the Tip Section 


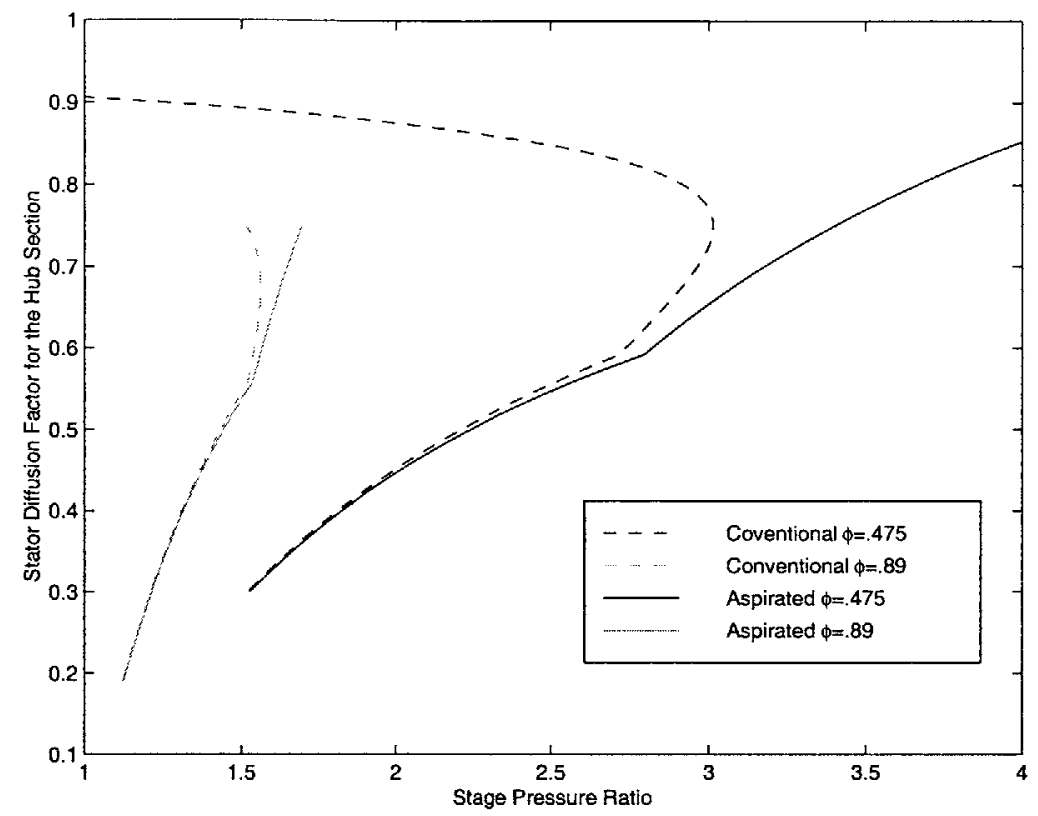

Figure D-11: Stator Diffusion Factor for the Hub Section

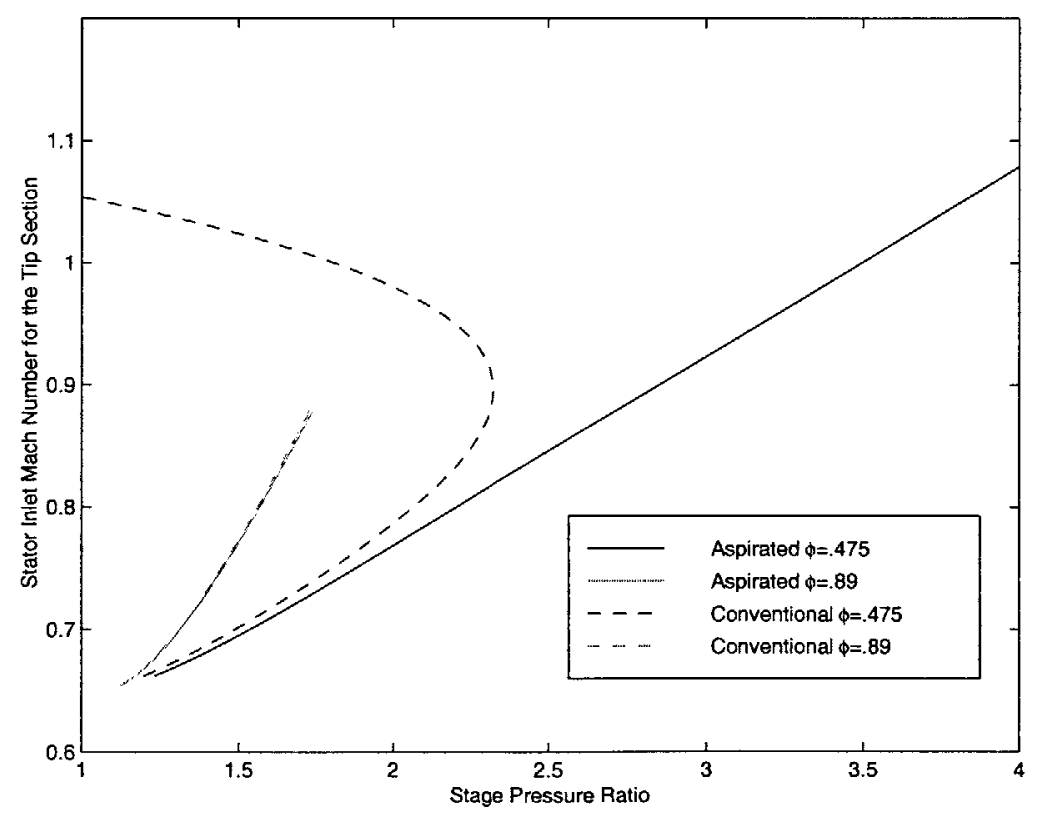

Figure D-12: Stator Inlet Mach number for the Tip Section 


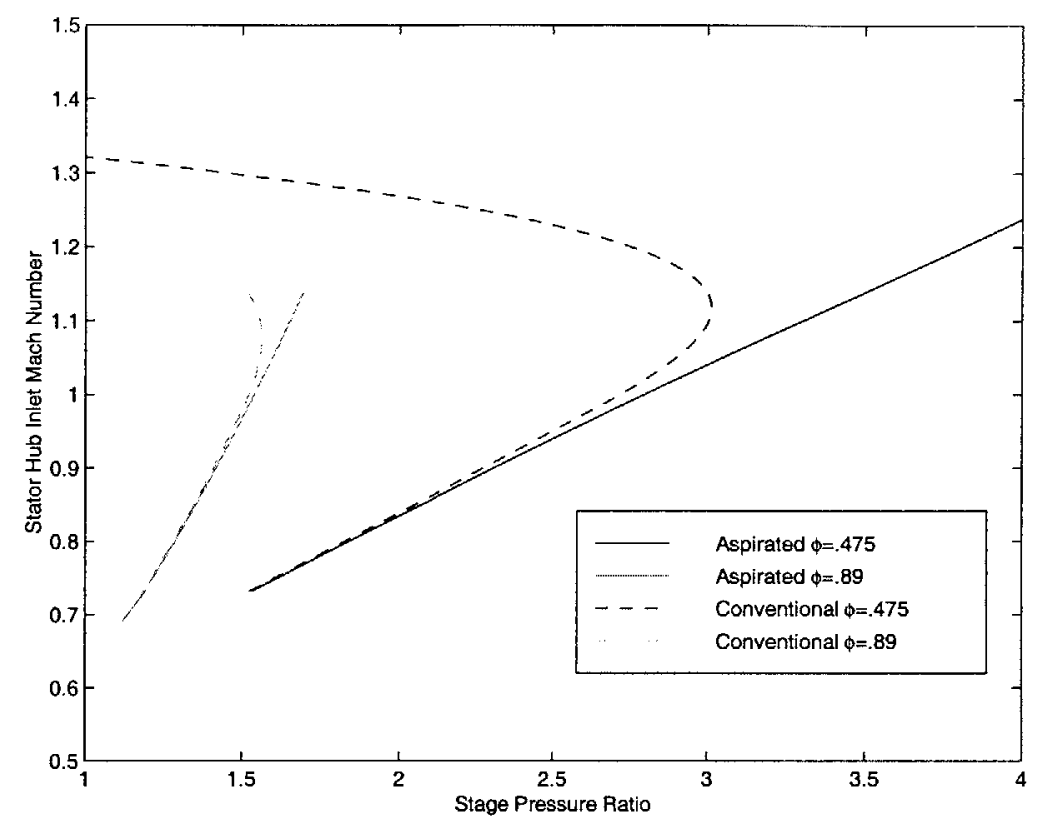

Figure D-13: Stator Inlet Mach number for the Hub Section

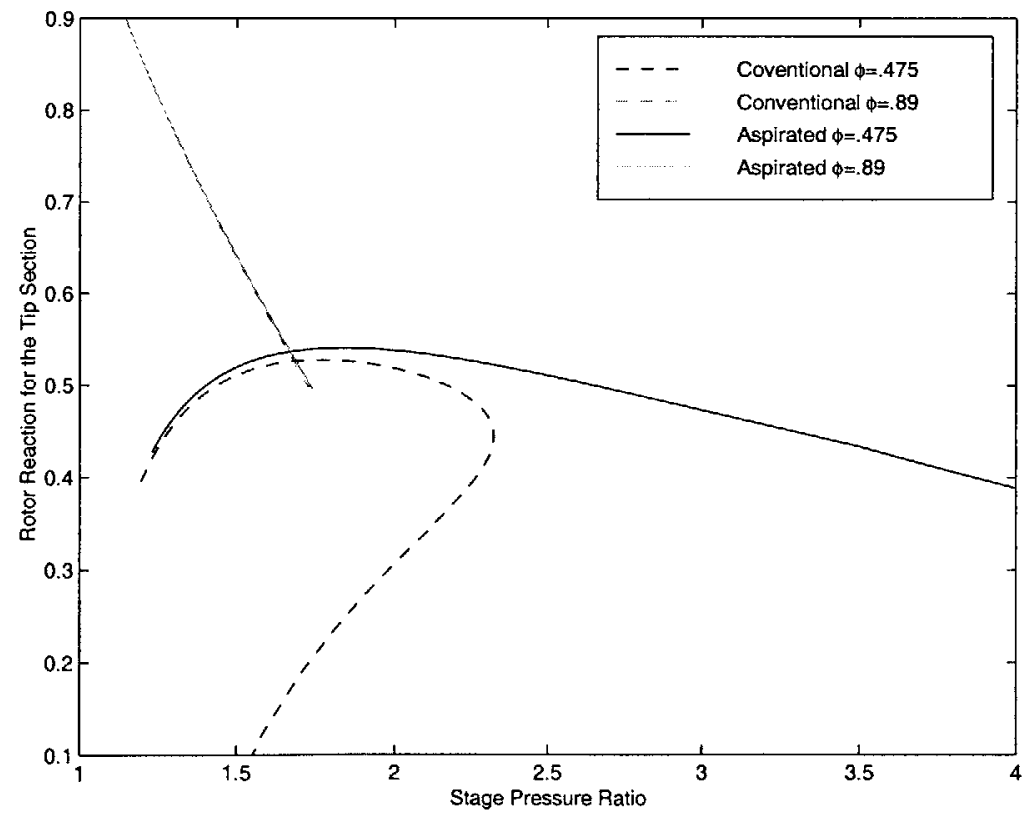

Figure D-14: Rotor Reaction for the Tip Section 


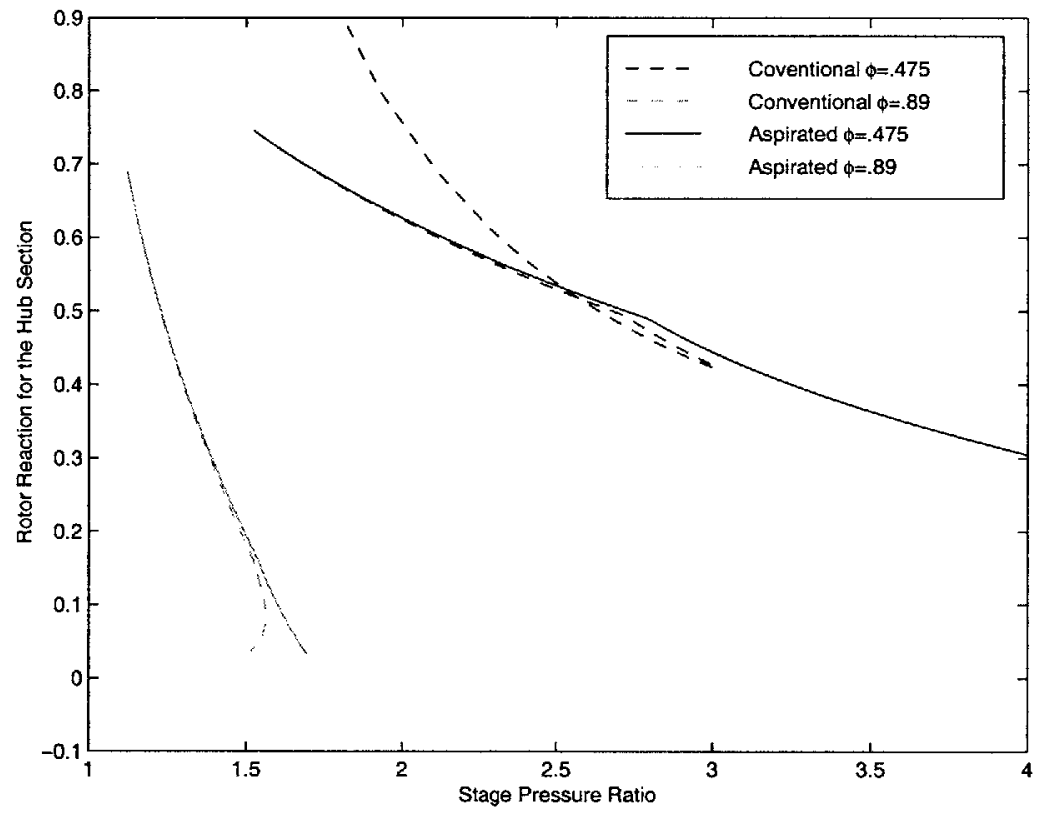

Figure D-15: Rotor Reaction for the Hub Section 


\section{D.2 Results}

Finally, using the radial variations and the shock-boundary layer interaction loss estimate, the effect of aspiration on the performance of different streamlines is shown directly. For the low speed conventional and aspirated stages, the limiting streamline for performance is the hub streamline due to the high swirl into the stator. The aspirated stage performs better because it controls the size of the boundary layer downstream of the shock impingement location. In the high speed stages, the conventional stage is limited by the tip streamline, in particular, the high relative Mach number and resulting large shock losses and shockboundary layer interaction losses. For the high speed aspirated stage, the hub streamline limits the performance, just as for the low speed case, due to the large Mach numbers incident on the stator hub section. 


\section{Bibliography}

[1] M. Drela and M.B. Giles. Viscous-inviscid analysis of transonic and low reynolds number airfoils. AIAA Journal, 25(10):1347-1355, 1987.

[2] Wing fai Ng. Detailed time-resolved measurements and analysis of unsteady flow in a transonic compressor. Technical Report GTL Report No. 150, Gas Turbine Lab, Massachusetts Institute of Technology, Cambridge, Massachusetts, 1980.

[3] Wing fai Ng. Time-resolved stagnation temperature measurement in a transonic compressor stage. Technical Report GTL Report No. 177, Gas Turbine Lab, Massachusetts Institute of Technology, Cambridge, Massachusetts, 1983.

[4] Jeffrey B. Gertz. Unsteady design-point flow phenomena in transonic compressors. Technical Report GTL Report No. 188, Gas Turbine Lab, Massachusetts Institute of Technology, Cambridge, Massachusetts, September 1986.

[5] J. L. Kerrebrock. The mit blowdown compressor facility. Technical Report 108, Massachusetts Institute of Technology, MIT Gas Turbine Lab, September 1975.

[6] Jack Kerrebrock. Aircraft Engines and Gas Turbines. The MIT Press, 2nd edition, 1992.

[7] J.L. Kerrebrock. The prospects for aspirated compressors. (AIAA 2000-2472), 2000.

[8] J.L. Kerrebrock, D.P. Reijnen, W.S. Ziminsky, and L.M. Smilg. Aspirated compressors. (ASME 97-GT525), 1997. 
[9] Petros A. Kotidis. Unsteady radial transport in a transonic compressor stage. Technical Report GTL Report No. 199, Gas Turbine Lab, Massachusetts Institute of Technology, Cambridge, Massachusetts, September 1989.

[10] S. Leiblein. Experimental flow in two-dimensional cascades. Technical Report SP-36, NASA, 1965. Chapter VI.

[11] R.J. Loughery, R.A. Horn, and P.C. Tramm. Single-stage experimental evaluation of boundary layer blowing and bleed techniques for high lift stator blades. NASA Contractor Report 54573, March 1971. 71N18749.

[12] Ali Merchant. Personal Communication, December 2000.

[13] Ali Merchant. Personal Communication, January 2001.

[14] Ali A. Merchant. Design and Analysis of Axial Aspirated Compressor Stages. PhD thesis, Massachusetts Institute of Technology, Cambridge, MA, June 1999.

[15] G. R. Miller, G. W. Lewis, and M. J. Hartmann. Shock losses in transonic rotor rows. ASME Journal of Engineering for Power, 83(3):235-242, 1961.

[16] J. A. Powell, A. J. Strazisar, and R. G. Seasholtz. Efficient laser anemometer for intra-rotor flow mapping in turbomachinery. ASME Journal of Engineering for Power, 103(2):424-429, April 1981.

[17] Duncan P. Reijnen. Experimental Study of Boundary Layer Suction in a Transonic Compressor. PhD thesis, Massachusetts Institute of Technology, Cambridge, MA, January 1997.

[18] V. Saxena and W. Lord. Personal Communication, 1999.

[19] Brian J. Schuler. Mechanical design of an experimental aspirated compressor. Master's thesis, Massachusetts Institute of Technology, Cambridge, MA, September 1998.

[20] Ascher H. Shapiro. The Dynamics and Thermodynamics of Compressible Fluid Flow, volume 1. The Ronald Press Company, 1953. 
[21] Om Sharma. Personal Communication, April 2001.

[22] L.M. Smilg. Design of a high pressure ratio fan stage to take advantage of boundary layer suction. Master's thesis, Massachusetts Institute of Technology, Cambridge, MA, 1994.

[23] A. J. Strazisar and J. A. Powell. Laser anemometer measurements in a transonic axial flow compressor rotor. ASME Journal of Engineering for Power, 103(2):430-437, April 1981.

[24] K.L. Tzuoo, S.S. Hingorani, and A.K. Sehra. Design methodology for splittered axial compressor rotors. (ASME 90-GT-66), 1990.

[25] Donald C. Urasek, William T. Gorrell, and Walter S. Cunnan. Performance of two-stage fan having low-aspect-ratio, first-stage rotor blading. Technical Report Technical Paper 1493, NASA, Lewis Research Center, Cleveland, OH, 1979.

[26] A.J. Wennerstrom. Experimental study of a high-throughflow transonic axial compressor stage. Journal of Engineering for Gas Turbines and Power, (106):552-559, 1984.

[27] A.J. Wennerstrom. Some experiments with a supersonic axial compressor. Journal of Turbomachinery, (109):388-397, 1987.

[28] A.J. Wennerstrom and et al. Investigation of a $1500 \mathrm{ft} / \mathrm{sec}$, transonic, high-throughflow, single-stage axial-flow compressor with low hub/tip ratio. Technical Report AFAPLTR-76-92, Air Force Aero Propulsion Laboratory, Wright Patterson Air Force Base, OH, October 1976.

[29] W.S. Ziminsky. Design of a high pressure ratio transonic compressor stage with active boundary layer control. Master's thesis, Massachusetts Institute of Technology, Cambridge, MA, 1996. 